\title{
WestVirginiaUniversity
}

THE RESEARCH REPOSITORY @ WVU

Graduate Theses, Dissertations, and Problem Reports

2017

\section{Molecular Simulation of Transport and Storage in Shale}

\author{
Mohammad Kazemi
}

Follow this and additional works at: https://researchrepository.wvu.edu/etd

\section{Recommended Citation}

Kazemi, Mohammad, "Molecular Simulation of Transport and Storage in Shale" (2017). Graduate Theses, Dissertations, and Problem Reports. 5950.

https://researchrepository.wvu.edu/etd/5950

This Dissertation is protected by copyright and/or related rights. It has been brought to you by the The Research Repository @ WVU with permission from the rights-holder(s). You are free to use this Dissertation in any way that is permitted by the copyright and related rights legislation that applies to your use. For other uses you must obtain permission from the rights-holder(s) directly, unless additional rights are indicated by a Creative Commons license in the record and/ or on the work itself. This Dissertation has been accepted for inclusion in WVU Graduate Theses, Dissertations, and Problem Reports collection by an authorized administrator of The Research Repository @ WVU.

For more information, please contact researchrepository@mail.wvu.edu. 


\title{
MOLECULAR SIMULATION OF TRANSPORT AND STORAGE IN SHALE
}

\author{
Mohammad Kazemi
}

\author{
Dissertation submitted to the \\ Benjamin M. Statler College of Engineering and Mineral Resources \\ at West Virginia University \\ in partial fullfillment of the requirements for the degree of \\ Doctor of Philosophy \\ in \\ Petroleum and Natural Gas Engineering
}

Ali Takbiri Borujeni, Chair Ph.D.

Shahab D. Mohaghegh , Ph.D.

Ebrahim Fathi, Ph.D.

Samuel Ameri, Ph.D.

Shikha Sharma, Ph.D.

Department of Petroleum and Natural Gas Engineering

Morgantown, West Virginia

2017

Keywords: Shale, Molecular Simulations, Kerogen, $\mathrm{CO}_{2}$ sequestration

Copyright (C) 2017 Mohammad Kazemi 


\title{
ABSTRACT
}

\section{Molecular Simulation of Transport and Storage in Shale}

\author{
Mohammad Kazemi
}

Over the past few years, the production of shale hydrocarbons has seen a renewed interest both in science and industry. Indeed, these fluids today constitute a significant energy and economic stake to compensate for the scarcity of so-called conventional resources. This is due to the fact that shale gases and oils represent enormous potential resources and are present all over the world.

In shales, hydrocarbons are generally contained in microporous organic nanopores: kerogen. The kerogen is both the source rock of hydrocarbons and their reservoir. In shales, the extreme confinement of fluids in organic matter, high pressure-high temperature thermodynamic condition as well as very low permeabilities, imply a significant change in the state of the fluids (present in adsorbed form) and its transport mechanisms (diffusive). In this dissertation, we studied the physical properties (adsorption, transport) of kerogen as well as its carbon dioxide sequestration potential.

The characteristic scales in the shales are of the order of a nanometer, which is accessible today by molecular simulations on supercomputers or even personal computers. Therefore, we have chosen to study kerogens by molecular simulation. The objective of this work is to stimulate a fundamental research on this subject in order to understand and model the mechanisms encountered in the shales and thus to respond responsibly and sustainably to the energy challenges of the years to come.

Initially, the simplified kerogen models (carbon nanochannels and nanocapillaries) are developed and transport and storage of different gases are studied. This part of research is beneficial for developing analytical models of gas transport in organic nanopores. Furthermore, kerogens with different maturities were generated by molecular dynamics simulations under thermodynamic conditions typical of this type of reservoir $(338 \mathrm{~K}, 20 \mathrm{MPa})$. In our simulations, the microporous network of kerogen is created by the inclusion of dummy particles, which were deleted after kerogen structure is created. The average density of the structures of organic matter created is in agreement with the experimental results obtained on such kerogens. The density is very strongly correlated with the stacking of the kerogenic polyaromatic clusters which is a strong indicator of the coherence of the simulated structures with respect to the experiments. We were interested in the transport of hydrocarbons in the kerogen and have identified the mechanisms of mass transfer through kerogens and we have been able to predict their evolution as a function of thermodynamic conditions (composition and pressure). Based on the results, it is demonstrated that the higher the maturity of kerogen, the higher is its adsorption capability. This is in agreement with experimental results of adsorption on kerogen. Furthermore, it is shown that the permeation of fluid through the kerogen membrane can be described by a diffusive formalism. The heavier alkanes have smaller diffusion coefficients and as a result, they may trap inside organic nanopores. Multicomponent diffusion of mixtures containing water and carbon dioxide is investigated and it is shown that water and carbon dioxide have lowest diffusion coefficients compared with hydrocarbons. The diffusion coefficients of hydrocarbons increases in presence of water due to higher adsorption capability of water and filling the adsorption sites. Adsorption molecular simulations of binary mixture of methane and carbon dioxide demonstrate that carbon dioxide have higher adsorption capabilities than methane. Binary mixture diffusion simulation of these two components also shows that carbon dioxide molecules have lower diffusion coefficients compared with methane. Therefore, injection of carbon dioxide into organic matter causes the methane molecules desorb and produce.

In conclusion, this dissertation work consisted of developing models, algorithms, and methodologies to predict the properties and mechanisms governing the behavior of the organic matter contained in the shales by employing molecular simulations. This work aims to improve our understanding of this type of resources. 
Those who listen to the Word, and follow the best of it. These are they whom God has guided. These are they who possess intellect.

(Quran 39:18)

To my wife, Elaheh

To my parents, Zabihollah and Maryam

To my parents-in-law, Hossein and Maryam

To my brother and his family, Mojtaba, Maryam, Zeinab, and Fatemeh

To my sister and her husband, Arefeh and Amin

To my sister-in-law, Mahsa 


\section{Acknowledgments}

There are no proper words to convey my deep gratitude and respect for my dissertation and research advisor, Dr. Ali Takbiri-Borujeni. He has inspired me to become an independent researcher and helped me realize the power of critical reasoning. He also demonstrated what a brilliant and hard-working scientist can accomplish. My sincere thanks must also go to the members of my thesis advisory and exam committee: Professors Samuel Ameri, Shahab D. Mohaghegh, Ebrahim Fathi, and Shikha Sharma. They generously gave their time to offer me valuable comments toward improving my work. In particular, Profs. Ebrahim Fathi and Shahab D. Mohaghegh provided me constructive criticism which helped me develop a broader perspective to my thesis. I am most grateful to the collaborators for lending me their expertise and intuition to my scientific and technical problems: Dr. Samuel Ameri and Dr. Shikha Sharma.

Special thanks must go to Dr. Kasra Fattah at Louisiana State University. Dr. Fattah taught me the basics of molecular simulations and always were there to help me.

I deeply thank my parents, Maryam Pourmohammadi and Zabihollah Kazemi for their unconditional trust, timely encouragement, and endless patience. It was their love that raised me up again when I got weary. My brother and his family, Dr. Mojtaba Kazemi, Maryam Alipour, Zeinab Kazemi, Fatemeh Kazemi, and my sister Arefeh Kazemi, have also been generous with their love and encouragement despite the long distance between us.

I would like to express my gratitude to my parents-in-law Hossein Parvizian and Maryam Jalali for their unfailing support. I also thank for heart-warming kindness from the my sister-in-law Mahsa Parvizian.

Finally, I thank with love to Elaheh Parvizian, my wife. Understanding me best as a Ph.D. herself, Elaheh has been my best friend and great companion, loved, supported, encouraged, entertained, and helped me get through this agonizing period in the most positive way. 


\section{Contents}

$\begin{array}{ll}\text { Abstract } & \text { ii }\end{array}$

Acknowledgments $\quad$ iv

List of Figures $\quad$ vii

List of Tables $\quad$ xi

1 Introduction 1

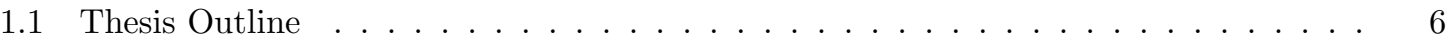

$\begin{array}{lll}2 & \text { Introduction to Molecular Simulations } & 7\end{array}$

2.1 The MD Algorithm . . . . . . . . . . . . . . . . . . . . 8

2.2 Molecular Interactions $\ldots \ldots \ldots \ldots \ldots$

$2.2 .1 \quad$ OPLS-All Atom Force Field . . . . . . . . . . . . . . . . . . . . 9

$2.2 .2 \quad$ COMPASS Force Field . . . . . . . . . . . . . . . . . . . . . . . . 10

2.3 Periodic Boundary Condition . . . . . . . . . . . . . . . . . . . . . 11

2.4 Statistical Ensembles . . . . . . . . . . . . . . . . . . . . . . . 11

3 Adsorption and Transport of Gases in Simplified Kerogen Model 12

3.1 DCV-GCMD Simulations in Organic Nanochannels . . . . . . . . . . . . . . . . . 14

3.1 .1 Computational Methodology _ . . . . . . . . . . . . . . . . . 15

3.1.2 Results and Discussion . . . . . . . . . . . . . . . . . . . 16

3.1.3 Chemical Potential Driven Flow . . . . . . . . . . . . . . . . . . . 18

3.1.4 Effect of Channel Length . . . . . . . . . . . . . . . . . . . . 24

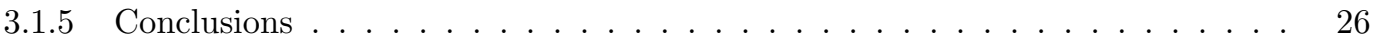

3.2 NEMD Simulations of Pure Gases in Organic Nanochannels . . . . . . . . . . . . . . 27 
3.2.1 Molecular Dynamics Methods . . . . . . . . . . . . . . . . 28

3.2.2 Results and Discussion . . . . . . . . . . . . . . . . . . 30

3.2.3 Conclusions .......................... . . 40

3.3 NEMD Simulations of Multicomponent Gases in Rough Organic Nanochannels . . . 41

3.3.1 Computational Methodology . . . . . . . . . . . . . . . . 42

3.3.2 Results and Discussion . . . . . . . . . . . . . . . . . . . 44

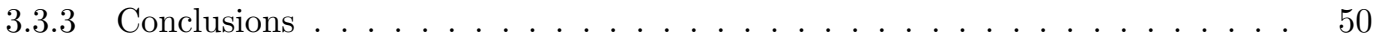

3.4 DCV-GCMD Simulations of Pure Gases in Smooth Organic Nanocapillaries . . . . . 52

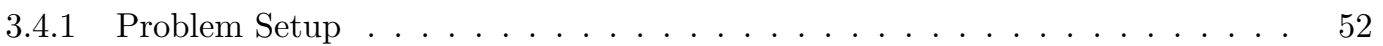

3.4.2 Results and Discussion . . . . . . . . . . . . . . . . . . . 53

3.4.3 Chemical Potential Driven Flow . . . . . . . . . . . . . . . . . 55

3.4.4 Comparison of R13 Model with Molecular Simulations . . . . . . . . . . . . . 61

3.4.5 Diffusion Coefficients . . . . . . . . . . . . . . . . . 65

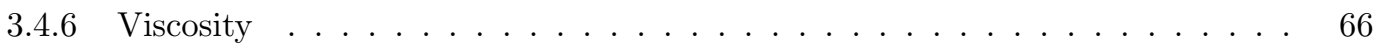

3.4 Conclusions .......................... 71

4 Carbon Dioxide Sequestration in Realistic Kerogen Model 73

4.1 Diffusion of Binary Mixtures . . . . . . . . . . . . . . . . . . . . . . . . 74

4.2 Binary Adsorption Isotherms . . . . . . . . . . . . . . . . . . . . . 76

4.3 Binary Diffusion Coefficients . . . . . . . . . . . . . . . . . . 86

5 Storage and Transport in Realistic Kerogen Model 90

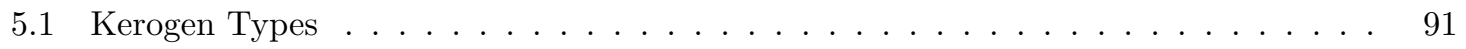

5.2 Creating Kerogen 3D structure . . . . . . . . . . . . . . . . . . . . . . . 92

5.3 Molecular Simulation Results and Discussions . . . . . . . . . . . . . . . . . . . 95

5.3 .1 Adsorption Isotherms . . . . . . . . . . . . . . . . . 95

5.3.2 Multicomponent Diffusion . . . . . . . . . . . . . . . . . 97

5.3.3 Significance of Multicomponent Diffusion _. . . . . . . . . . . . . . 101

6 Conclusions $r$\begin{tabular}{l}
107 \\
\hline
\end{tabular} 


\section{List of Figures}

2.1 Lennard-Jones potential interactions of different gases with carbon in graphite as a function of dimensionless distance. . . . . . . . . . . . . . . . . . . . . . 9

3.1 Simulation setup for the $2 \mathrm{~nm}$ channel. The channel length is $40 \mathrm{~nm}$. "H" and "L" represent the high and low pressure reservoirs, respectively. . . . . . . . . . . . 15

3.2 Absolute adsorption isotherms of argon, methane, helium, and neon for 2 and $5 \mathrm{~nm}$ channel heights. . . . . . . . . . . . . . . . . . . . . . . 17

3.3 Excess adsorption isotherms of argon, methane, helium, and neon in 2 and $5 \mathrm{~nm}$ channel heights. . . . . . . . . . . . . . . . . . . . . . . 18

3.4 Average velocities across the channel height vs. pressure gradient. Simulations are performed in the linear response regime. . . . . . . . . . . . . . . . . . . . 18

3.5 Lennard-Jones interaction between different gases and organic channel. . . . . . . . 20

3.6 Density and normalized velocity profiles of argon and methane in the $2 \mathrm{~nm}$ channel. 20

3.7 Density and normalized velocity profiles of helium and neon in the $2 \mathrm{~nm}$ channel. . . 21

3.8 Density and normalized velocity profiles of argon and methane in the 5 nm channel. 22

3.9 Density and normalized velocity and profiles of helium and neon in the $5 \mathrm{~nm}$ channel. 22

3.10 Dimensionless mass flux profile of methane in the 2 and $5 \mathrm{~nm}$ channels. The profiles are plotted for the channel half height. . . . . . . . . . . . . . . . . . . 23

3.11 Sensitivity of the velocity of methane molecules to channel length for a $2 \mathrm{~nm}$ channel. 26

3.12 Molecular dynamics simulation geometry. The figure is not in scale. . . . . . . . . 30

3.13 Adsorption isotherms of Argon, Methane, and Helium at 2 and $4 \mathrm{~nm}$ height channel at 300 K. (a) Absolute adsorption (b) Excess adsorption . . . . . . . . . . . . . . . . 31

3.14 Relationship between the average density and pressure of Argon for the 2- $n m$ channel. 32

3.15 Velocity and density profiles of Methane in the graphite channels. . . . . . . . . . . 34 
3.16 Velocity and density profiles of Argon in the graphite channels. . . . . . . . . . . . 34

3.17 Velocity and density profiles of Helium in the graphite channels. . . . . . . . . . 35

3.18 Molecular flux vs. pressure gradient for (a) 2 and $4 \mathrm{~nm}$, (b) $6 \mathrm{~nm}$, and (c) $8 \mathrm{~nm}$. . . 37

3.19 Dimensionless mass flux profiles of Argon, Methane, and Helium at (a) Kn=0.1 (b)

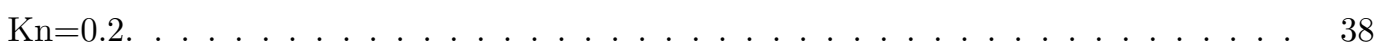

3.20 Transport diffusion coefficients at (a) $\mathrm{Kn}=0.1$ and (b) $\mathrm{Kn}=0.2 \ldots \ldots \ldots$

3.21 Representation of the rough surfaces used in the simulations. . . . . . . . . . . . 42

3.22 Density profiles of the two samples in (a) $2 \mathrm{~nm}$ and (b) $4 \mathrm{~nm}$ channels. . . . . . . 45

3.23 Density profiles of each gas component in the two samples in $2 \mathrm{~nm}$ and $4 \mathrm{~nm}$ channels. 45

3.24 Selectivity of hexane over other gas components in (a) $2 \mathrm{~nm}$ and (b) $4 \mathrm{~nm}$ channels. 46

3.25 Velocity profiles of the gas samples in 2 and 4-nm channels. (a, b) Sample 1 and (c,

d) Sample $2 . \ldots \ldots \ldots \ldots \ldots \ldots$

3.26 The molecular fluxes across the 2 and $4 \mathrm{~nm}$ channels for both samples. . . . . . . . 49

3.27 Diffusion coefficients of each gas component in the gas samples for 2- and 4-nm channels. 50

3.28 Simulation setup for the $2 \mathrm{~nm}$ capillary. The capillary length is $40 \mathrm{~nm}$. "H" and "L" represent the high and low pressure reservoirs, respectively. . . . . . . . . . . 53

3.29 Absolute adsorptions for the (a) $2 \mathrm{~nm}$ and (b) $4 \mathrm{~nm}$ capillaries. . . . . . . . . 54

3.30 Excess adsorptions for a (a) $2 \mathrm{~nm}$ and (b) $4 \mathrm{~nm}$ capillaries. . . . . . . . . . . 55

3.31 Density and normalized velocity profiles of Argon. . . . . . . . . . . . . . 56

3.32 A comparison of calculated mass flux with the ones predicted by Knudsen diffusion.

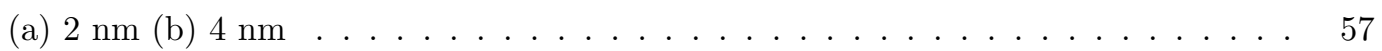

3.33 Density and normalized velocity profiles of Methane. . . . . . . . . . . . . 58

3.34 A comparison of computed mass flux for methane with the ones predicted by Knudsen diffusion. (a) $2 \mathrm{~nm}$ (b) $4 \mathrm{~nm} \ldots \ldots \ldots \ldots \ldots \ldots$

3.35 Density and normalized velocity profiles of Neon. . . . . . . . . . . . . . . . . 59

3.36 A comparison of COMPUTED mass flux values for neon with the ones predicted by Knudsen diffusion. (a) $2 \mathrm{~nm}$ (b) $4 \mathrm{~nm} \ldots \ldots \ldots \ldots \ldots$

3.37 Lennard-Jones potential of studied gases. . . . . . . . . . . . . . . . . . 60

3.38 Comparison of the simulation results with R13 model for argon in the $2 \mathrm{~nm}$ capillary (a) $\mathrm{P}_{\text {avg }}=100 \mathrm{~atm}, \Delta \mathrm{P}=100 \mathrm{~atm}$, (b) $\mathrm{P}_{\text {avg }}=50 \mathrm{~atm}, \Delta \mathrm{P}=60$ atm, and (c) $\mathrm{P}_{\text {avg }}$

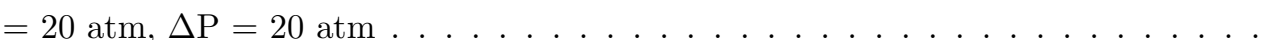


3.39 Comparison of the simulation results with R13 model for methane in the $2 \mathrm{~nm}$ capillary (a) $\mathrm{P}_{\text {avg }}=100 \mathrm{~atm}, \Delta \mathrm{P}=100 \mathrm{~atm}$, (b) $\mathrm{P}_{\text {avg }}=50 \mathrm{~atm}, \Delta \mathrm{P}=60 \mathrm{~atm}$, and (c) $\mathrm{P}_{\text {avg }}=20 \mathrm{~atm}, \Delta \mathrm{P}=20 \mathrm{~atm} \ldots \ldots \ldots \ldots$

3.40 Comparison of the simulation results with R13 model for argon in the $4 \mathrm{~nm}$ capillary (a) $\mathrm{P}_{\text {avg }}=100 \mathrm{~atm}, \Delta \mathrm{P}=100 \mathrm{~atm}$, (b) $\mathrm{P}_{\text {avg }}=50 \mathrm{~atm}, \Delta \mathrm{P}=60 \mathrm{~atm}$, and (c) $\mathrm{P}_{\text {avg }}$

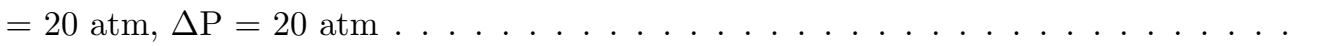

3.41 Comparison of the simulation results with R13 model for methane in the $4 \mathrm{~nm}$ capillary (a) $\mathrm{P}_{\text {avg }}=100 \mathrm{~atm}, \Delta \mathrm{P}=100 \mathrm{~atm}$, (b) $\mathrm{P}_{\text {avg }}=50 \mathrm{~atm}, \Delta \mathrm{P}=60 \mathrm{~atm}$, and (c) $\mathrm{P}_{\text {avg }}=20 \mathrm{~atm}, \Delta \mathrm{P}=20 \mathrm{~atm} \ldots \ldots \ldots \ldots$

3.42 The product of TMAC and adsorption surface coverage, $\theta$, as a function of Knudsen

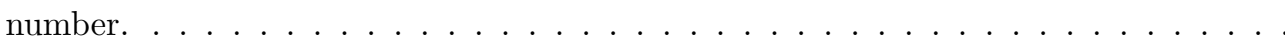

3.43 Transport and corrected diffusivity coefficients for argon and methane. (a) $2 \mathrm{~nm}$ (b)

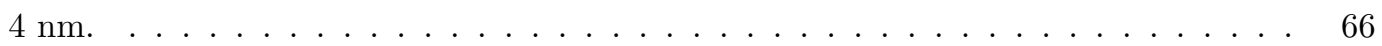

3.44 Thermodynamic factors for argon and methane in the 2 and $4 \mathrm{~nm}$ capillaries. . . . 66

3.45 Self-diffusion coefficient of methane and argon in the carbon nanotubes with 2 and 4 nm diameters; (a) Argon (b) Methane . . . . . . . . . . . . . . . . . . . . . . 68

3.46 Viscosity of gases in the 2 and $4 \mathrm{~nm}$ capillaries as a function of fugacity; (a) Argon (b) Methane (c) Neon. . . . . . . . . . . . . . . . . . . . . . . . .

3.47 Viscosity of gases in the 2 and $4 \mathrm{~nm}$ capillaries as a function of Knudsen number; (a) Argon (b) Methane (c) Neon. . . . . . . . . . . . . . . . . . . . . . . . . 70

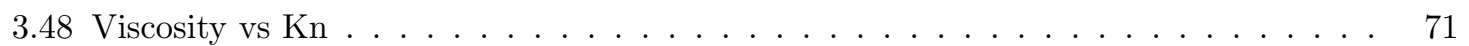

4.1 Binary adsorption isotherms of $\mathrm{CH}_{4}$ and $\mathrm{CO}_{2}$ for kerogen types IIA, IIB, and IIC. . $\quad 77$

4.2 Binary adsorption isotherms of $\mathrm{CH}_{4}$ and $\mathrm{CO}_{2}$ for kerogen types IID and IIIA. . . . 78

4.3 Comparison between the simulation results and fits using Eqn. 4.9 for the binary mixture adsorption for $\mathrm{CH}_{4}$ (red open circles) and $\mathrm{CO}_{2}$ (filled circles). The line

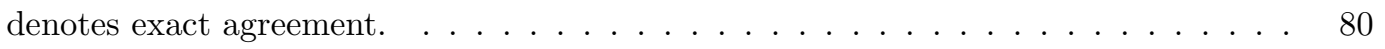

4.4 Selectivities and $\mathrm{x}-\mathrm{y}$ diagrams at different total pressures for kerogen type IIA. . . . 82

4.5 Selectivity values of different kerogen types at 50-50\% gases composition. . . . . . . 82

4.6 Selectivities and $\mathrm{x}-\mathrm{y}$ diagrams at different total pressures for kerogen type IIB. . . . 83

4.7 Selectivities and $\mathrm{x}-\mathrm{y}$ diagrams at different total pressures for kerogen type IIC. . . . 84

4.8 Selectivities and x-y diagrams at different total pressures for kerogen type IID. . . . 85

4.9 Selectivities and x-y diagrams at different total pressures for kerogen type IIIA. . . 86 
4.10 Onsager diffusion coefficients of $\mathrm{CH}_{4}-\mathrm{CO}_{2}$ mixtures in the channel; (a) $L_{11}$ (b) $L_{12}$

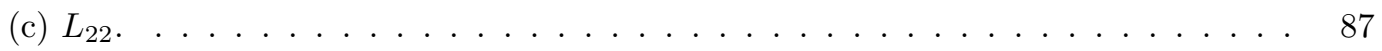

4.11 Onsager diffusion coefficients of $\mathrm{CH}_{4}-\mathrm{CO}_{2}$ mixtures in the channel; (a) $L_{11}$ (b) $L_{12}$

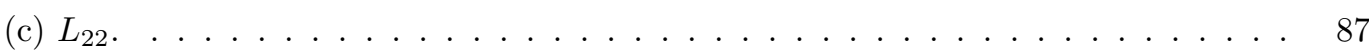

4.12 Onsager diffusion coefficients of $\mathrm{CH}_{4}-\mathrm{CO}_{2}$ mixtures in the channel; (a) $L_{11}$ (b) $L_{12}$

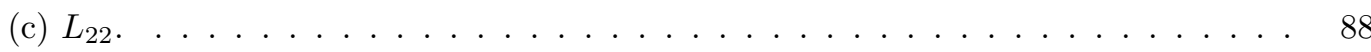

4.13 Onsager diffusion coefficients of $\mathrm{CH}_{4}-\mathrm{CO}_{2}$ mixtures in the channel; (a) $L_{11}$ (b) $L_{12}$

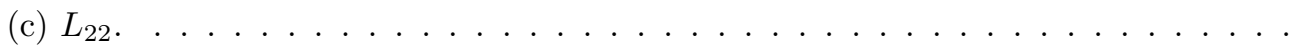

4.14 Onsager diffusion coefficients of $\mathrm{CH}_{4}-\mathrm{CO}_{2}$ mixtures in the channel; (a) $L_{11}$ (b) $L_{12}$

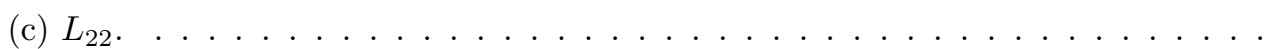

5.1 Van Krevelen diagram of the kerogen samples $[60,104] \ldots \ldots$. . . . . . . . . . . 92

5.2 Unit cells of kerogen with different maturities. Color code: cyan is for carbon, silver for hydrogen, red for oxygen, blue for nitrogen, and yellow for sulfur. . . . . . . . . 93

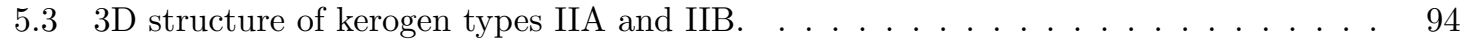

5.4 Adsorption isotherms of kerogens with different maturities along with experimental data of Rexer et al. [82] and Zhang et al. [112] . . . . . . . . . . . . . . . 96

5.5 Matrices of Onsager coefficients for kerogen type IIA for both mixtures. . . . . . . . 99

5.6 Matrices of Onsager coefficients for kerogen type IIB for both mixtures. . . . . . . . . 99

5.7 Matrices of Onsager coefficients for kerogen type IIC for both mixtures. . . . . . . . 100

5.8 Matrices of Onsager coefficients for kerogen type IID for both mixtures. . . . . . . . 100

5.9 Matrices of Onsager coefficients for kerogen type IIIA for both mixtures. . . . . . . 101

5.10 The diagonal components of Onsager matrix of diffusion coefficients determined using EMD simulations. . . . . . . . . . . . . . . . . . . . . . . . . 101

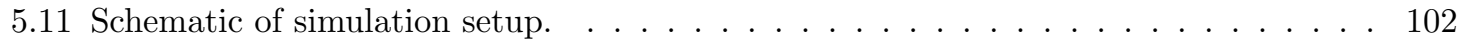

5.12 Diffusion of methane at different simulation times. . . . . . . . . . . . . . . . 104

5.13 Diffusion of ethane at different simulation times. . . . . . . . . . . . . . . 105

5.14 Diffusion of carbon dioxide at different simulation times. . . . . . . . . . . . . . . 105

5.15 Diffusion of water at different simulation times. . . . . . . . . . . . . . . 106

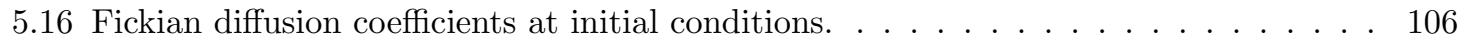




\section{List of Tables}

3.1 Lennard-Jones parameters . . . . . . . . . . . . . . . . . . . . . . 16

3.2 Transport diffusion coefficients for methane, argon, helium, and neon for all the channel sizes. . . . . . . . . . . . . . . . . . . . . . . . . 2 25

3.3 Applied forces to the inlet region of the channels for different gases at Knudsen num-

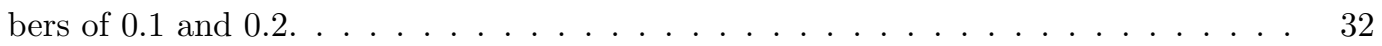

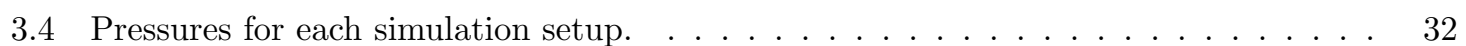

3.5 Transport diffusion coefficients for Methane, Argon, and Helium at Kn=0.1 and Kn0.2 for all the channel sizes. . . . . . . . . . . . . . . . . . . . . . 39

3.6 Composition of the gas samples used for simulations. . . . . . . . . . . . . . . 43

3.7 Selectivities of hexane over other gas components for two samples used for simulations. 46

3.8 Diffusion coefficients of different gas components in the gas samples. . . . . . . . . . 51

3.9 The Knudsen numbers and pressures under which the molecular simulations are per-

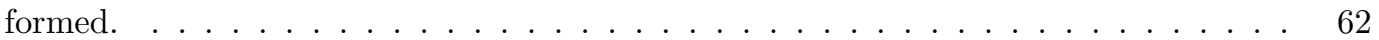

4.1 Calculated parameters for the binary adsorption isotherms (Equation. 4.9 ) $\ldots$. . 79

5.1 Mass and chemical formula of kerogens with different maturities. . . . . . . . . . . . 92

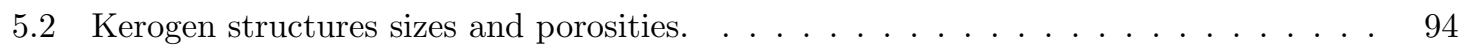

5.3 The Langmuir constants for different kerogen maturities. . . . . . . . . . . . . . . 97

5.4 Gas composition used for multicomponent diffusion. . . . . . . . . . . . . . . 97 


\section{Chapter 1}

\section{Introduction}

The United States' Natural gas production from shale resources increased from 4 percent of total gas production in 2005 to $40 \%$ in 2012, and is expected to rise to $53 \%$ in 2040. [105]. Shale is different from conventional hydrocarbon resources due to presence of nanoporous organic matter (kerogen) embedded within the inorganic matrix. Due to the nano-scale sizes of the pores in kerogen, transport of gases in kerogen deviates from continuum-based on which Navier Stokes and Darcy equation are derived. The pore sizes become comparable to the mean free path of the molecules, which causes non-continuum flow regimes. Furthermore, depending on the gas type and intermolecular forces between the gas molecules and solid molecules at the pore wall, the gas molecules in the vicinity of the pore wall cannot escape from the force field at the pore wall. The tendency of gas molecules to be adsorbed to the surfaces of the organic pores results in a heterogeneous fluid distributions within the pores. Adsorption of gas molecules to the pore surfaces causes molecular level transport effects, such as surface-diffusion. Therefore, experimental measurement of the transport properties of the shale core samples, if performed with gases different from the gases originally present in the sample, might result in different values of the transport constants. Without accurate measurement of the transport coefficients, accurate prediction of the initial hydrocarbon in place and also recovery from these resources remain limited [23, 44, 67].

Gas flow regimes are characterized by the Knudsen number, $K n$, which is the ratio of the gas mean free path to the characteristic length of the media. Flow regimes consist of continuum flow 
$\left(0<K n<10^{-3}\right)$, slip flow $\left(10^{-3}<K n<10^{-1}\right)$, transition flow $\left(10^{-1}<K n<10\right)$, and free molecular flow $(10<K n<\infty)$ [86]. In kerogen pores, the Knudsen number is larger than 0.1 [50] and gas flow usually falls into the slip or early transition flow regime $[21,95]$. Furthermore, due to the attraction forces between gas molecules and solid molecules at the pore wall, density of the gas molecules at the pore surface becomes higher than the middle of the pore; there is heterogeneous density distribution of fluids in the pore. The adsorbed gas phase may contribute to the mass flux across the channel. Therefore, there is a functional relationship between the surface coverage (or adsorbed gas density) and transport of the gases in the pores.

Several attempts have been made to develop mathematical models for flow in extremely low permeability porous media while accounting for rarefaction effects. Klinkenberg [64] took into account the slip effect and experimentally found that the apparent gas permeability is approximately a linear function of the reciprocal mean pressure. Beskok and Karniadakis [9] presented a formulation for flow through a capillary tube having a circular cross-section based on the Navier-Stokes equations. Due to non-equilibrium behavior of the gas on length scales of the order of mean free path, the Navier-Stokes equations fail to describe flows in rarefied gases [99]. Zhu et al. [115] showed that the measured gas permeability in tight porous media with $K n>0.1$ does not follow the Klinkenberg equation and recommended a higher-order equation. Civan [22] developed correlations for Klinkenberg slippage factor as well as tortuosity. Other authors also proposed correlations for Klinkenberg slippage factor for tight porous mediums [34, 48, 91]. Tang et al. [101] developed a Lattice Boltzmann Method (LBM) for gaseous slip flow in microscale pore geometries; however, this method is appropriate for flows with moderate Knudsen numbers $(K n \sim 0.1)$ when using the kinetic boundary conditions or maximizing an entropy.

Javadpour [44] used diffusive-advective gas flow model to develop an analytical model for apparent permeability of the nanotubes. The total mass flux is determined by superposition of the Knudsen diffusion and advective gas flow driven by pressure forces. Knudsen diffusion term is derived by fully diffusive gas-wall interaction assumption [70]; in the pressure force term, on the contrary, gas-wall interactions can be adjusted by changing the Tangential Momentum Accommodation Coefficient (TMAC). Beskok and Karniadakis [9] used the Navier-Stokes equations with second order slip boundary conditions and developed an equation for volumetric gas flow through single pipe for $K n<0.5$ (unified flow model). Civan [22] employed the Beskok and Karniadakis [9] model to develop an equation for apparent gas permeability. This model underestimates the apparent permeability due to the nonlinear behavior of gas near the wall that cannot be accounted for using 
Navier-Stokes equations.

Several numerical and experimental models for apparent permeability have also been proposed $[2,22$, 34, 48, 90, 91, 101, 115]. The contribution of the surface roughness added to the Javadpour [44] model by Darabi et al. [27]. The NAP model which was recently developed by Singh et al. [94] is claimed to be a nonempirical model for ultratight porous media. In this model, the no slip flow is assumed to remove the apparent permeability dependency on Tangential Momentum Accommodation Coefficient (TMAC). However, the Knudsen diffusion term used in this model contains the assumption of fully diffusive boundary condition (TMAC=1) [70].

The Boltzmann equation is valid for rarefied gas flows for all ranges of Knudsen number; however, the numerical simulation of the Boltzmann equation is computationally expensive. In order to overcome this problem, the Chapman-Enskog method $[17,19]$ and the method of moments of Grad [35] were developed. Unfortunately, the Chapman-Ensgok method, in which the phase density is expanded in powers of the Knudsen number, becomes linearly unstable for the second and third order expansions (Burnett and Super-Burnett) and can not be used for simulation of processes with small wavelengths $[13,85,97]$. In the method of moments of Grad [97], the Boltzmann equation is transfered to a set of partial differential equations for the moments of differential equations (set of moment equations). Although these methods are valid for higher ranges of Knudsen number, they do not consider adsorption and desorption of gases to pore surfaces.

Molecular dynamics is an effective tool to study the details of fluid-fluid and fluid-solid interactions in nano-scale conduits. Transport of gas molecules in microporous systems have been extensively studied using three different MD simulation methods: equilibrium molecular dynamics (EMD), external field Non-Equilibrium Molecular Dynamics (NEMD), and boundary driven non-equilibrium molecular dynamics (dual control volume grand canonical molecular dynamics or DCV-GCMD)[4].

DCV-GCMD simulations have been used to investigate the sensitivity of transport properties of methane, carbon dioxide, and nitrogen to pore size, porosity, and pressure gradient [33]. Jin and Firoozabadi [47] investigated flow of Methane in carbon nanotubes using DCV-GCMD simulations and the mobility of adsorbed phase in high and low pressures were demonstrated. They found that the Knudsen diffusion and Hagen-Poiseuille equations can underestimate the Methane flow by one order of magnitude. [59] studied the transport of four different gases, Methane and Argon (adsorbing) and Helium and Neon (less-adsorbing). They showed that as the average channel pressure increases, the contribution of the adsorbed phase to the total mass flux decreases. For organic nanocapillaries, 
DCV-GCMD simulations have shown that the Knudsen diffusion model underestimates the molecular flux [57]. This underestimation was not a strong function of the pore size or the pore pressure.

NEMD simulations are easy to implement and computationally efficient for simulating the transport phenomena; however, the equivalence of external forcing function that drives diffusion and the actual chemical potential gradient has not been formally demonstrated [4]. NEMD simulations have been applied to investigate the slippage of a non-adsorbing gas (Helium) in microporous media [32].

Feng et al. [31] carried out NEMD and DCV-GCMD to study the flow of Methane in nano-capillaries. They showed that the surface transport of adsorbed Methane have a significant contribution to total mass flux in nano-capillaries.

Comparing the transport properties of different types of gases in nano-scale conduits using NEMD is challenging because these systems have many degrees of freedom, e.g., channel size, molecule size, gas density, and therefore, finding equivalent systems is difficult. Furthermore, for systems under confinement, there is a positional dependence of density and also the state variables, pressure and temperature [103].

There have been some studies on the adsorption and transport of multi-component hydrocarbon mixtures in shale organic matter using MD. Ma and Jamili [68] used Simplified Local-Density theory coupled with Modified Peng-Robinson Equation of State to predict the density profiles of pure and mixture hydrocarbons in confined pores. They found that the compositions of the fluid mixtures are not uniformly distributed across the pore. Heavier component (n-butane) tended to accumulate near the wall while lighter component (methane) would tend to stay in the center region of the pore. Collell et al. [25] used EMD and boundary driven non-equilibrium molecular dynamics (BDNEMD) simulations to study the transport of fluid mixtures for different chemical compositions through a molecular model of kerogen type II. They computed Onsagers coefficients for pure and multi-component mixture of fluids. The results suggested that the flow inside kerogen is of diffusive nature and that the transport properties can be calculated using EMD.

The importance of understanding gases flow in organic nanopores is not only limited to predicting production from shale resources but also it is significant in understanding the capability of organic matter for carbon dioxide sequestration. Carbon capture and sequestration (CCS) in depleted shale gas reservoirs provides an opportunity for underground storage of the $\mathrm{CO}_{2} \cdot \mathrm{CO}_{2}$ has higher affinity to the organic matter of shale compared to $\mathrm{CH}_{4}$. Injecting $\mathrm{CO}_{2}$ into the shale reservoirs can cause the $\mathrm{CH}_{4}$ molecules in the adsorbed state to be desorbed and and replaced by the $\mathrm{CO}_{2}$ molecules 
(enhanced gas recovery-EGR). Current understanding of the competitive adsorption/desorption process of $\mathrm{CO}_{2}$ and $\mathrm{CH}_{4}$ and its impact on the efficiency of CCS is still insufficient. Furthermore, the transport coefficients describing this process are not well defined.

In EGR and CCS processes in shale, the competitive adsorption equilibia is of significant importance. There have been many approaches to determine the adsorption isotherms of pure $\mathrm{CO}_{2}$ and pure $\mathrm{CH}_{4}$ to estimate the maximum capacity for $\mathrm{CO}_{2}$ storage and $\mathrm{CH}_{4}$ recovery including the volumetric method [40, 71, 77], manometric method [62, 111], and gravimetric method [80]. Adsorption of binary $\mathrm{CO}_{2}-\mathrm{CH}_{4}$ mixtures has also been measured using gravimetric-chromatographic method [79].

Molecular simulations have been proved to be credible alternatives to experimental measurements of species adsorption in micoroporous materials [4]. GCMC simulations have been extensively used to study the pure species adsorption in microporous carbon [12], and realistic molecular models of coal [113] and shale [25] and species mixtures in microporous carbon [12].

There are few studies on species diffusion in EGR process in the literature. There are a few mechanisms, such as inhomogeneous fluid distribution of the species, adsorption selectivity, and apparent viscosity that manifest themselves into different diffusion coefficients, namely self-, corrected (or Maxwell-Stefan), and transport diffusion coefficients. The transport diffusion coefficients of pure $\mathrm{CO}_{2}$ and pure $\mathrm{CH}_{4}$ and $\mathrm{CO}_{2}-\mathrm{CH}_{4}$ mixtures can be calculated from their trajectories [113].

The objective for this work is to shed light on the the diffusion phenomena of the pure and binary mixtures by performing molecular simulations in carbon-based organic nanochannels as well as kerogen 3D structures with different maturities.

Initially, the simplified kerogen models (carbon nanochannels and nanocapillaries) are developed and transport and storage of different gases are studied. This part of research is beneficial for developing analytical models of gas transport in organic nanopores. Furthermore, kerogens with different maturities were generated by simulations of molecular dynamics under thermodynamic conditions typical of this type of reservoir $(338 \mathrm{~K}, 20 \mathrm{MPa})$. In our simulations, the microporous network of kerogen is created by the inclusion of dummy particles, which were deleted after kerogen structure is created. The average density of the structures of organic matter created is in agreement with the experimental results obtained on such kerogens. This is a strong indicator of the coherence of the simulated structures with respect to the experiments, the density being very strongly correlated with the stacking of the kerogenic polyaromatic clusters.

We were interested in the transport of hydrocarbons in the kerogen and have identified the mech- 
anisms of mass transfer through kerogens and we have been able to predict their evolution as a function of thermodynamic conditions (composition and pressure). Based on the results, it is shown that the permeation of fluid through the kerogen membrane can be described by a diffusive formalism. The heavier alkanes have smaller diffusion coefficients and as a result, they may trap inside organic nanopores.

\subsection{Thesis Outline}

This dissertation work consisted of developing models,algorithms and methodologies to predict by molecular simulations the properties and mechanisms governing the behavior of the organic matter contained in the shales. This work aims to improve our understanding of this type of resources. Although these developments have been applied to the shales only theme, they are also part of the more general framework of the modeling of amorphous microporous materials.

Chapter 2 outlines the fundamental principles of molecular simulations. Molecular simulations of transport and storage in simplified organic nanopores (graphite nanochannels and nanocapillaries) are presented. In chapter 4 , binary adsorption and diffusion of methane and carbon dioxide is investigated with application to enhanced gas recovery. Chapter 5 is devoted to creating $3 \mathrm{D}$ structures of kerogens with different maturities and studying the hydrocarbon transport and storage. Chapter 6 presents a summary and brief discussions of the results presented in the previous chapters and also addresses future research that may be fruitful to other researchers. 


\section{Chapter 2}

\section{Introduction to Molecular}

\section{Simulations}

Molecular simulations are strong tools that are fruitful for many complex problems. They are performed to study the behavior of ensemble of molecules that are hard to investigate using experiments. For example, molecular simulations could help in studying properties of a material that cannot be isolated easily or it is a good substitute for experiments that require high temperature and high pressure environments. The molecular simulations can be used as a complement to experiments [3]. There are two main families of molecular simulations, i.e. molecular dynamics and Monte Carlo. In molecular dynamic, materials are considered as a collection of atoms which exert forces to each other defined by interaction potentials. The atoms in system move according to Newton's equation of motion. The equation of motion is solved numerically for all atoms in system which for a simple system may be written,

$$
m_{i} \frac{\partial^{2} \boldsymbol{r}_{\boldsymbol{i}}}{\partial t^{2}}=\boldsymbol{f}_{\boldsymbol{i}}, \quad \boldsymbol{f}_{\boldsymbol{i}}=\frac{\partial}{\partial \boldsymbol{r}_{\boldsymbol{i}}} U
$$

where $\boldsymbol{f}_{\boldsymbol{i}}$ is force, $m$ is atomic mass, and $\boldsymbol{r}_{\boldsymbol{i}}$ is position of atom $i$. The forces between atoms depend on position by interaction potential $\left(U\left(r^{N}\right)\right.$ where $\left.r^{N}=\left(r_{1}, r_{2}, \ldots r_{N}\right)\right)$. The molecular dynamics method provide dynamic, structural and thermodynamic properties.

On the other hand, the main idea behind the Monte Carlo simulations is importance sampling. In Monte Carlo simulations, the dynamics of system does not mimic the dynamics of real system; however, the equilibrium properties of the system should correspond to the real system's equilibrium 
properties.

\subsection{The MD Algorithm}

In order to simulate the motion of molecules, Eq. 2.1 needs to be solved numerically for all molecules in system. The Verlet algorithm is usually used in Molecular simulation codes. In this algorithm, the velocity term is eliminated by comparing two Taylor expansions about position at time $t$. The Verlet algorithm starts by approximating $\frac{\partial^{2} \boldsymbol{r}_{\boldsymbol{i}}}{\partial t^{2}}$

$$
\frac{\partial^{2} \boldsymbol{r}_{\boldsymbol{i}}}{\partial t^{2}}=\frac{r(t+\Delta t)-2 r(t)+r(t-\Delta t)}{\Delta t^{2}}
$$

Thus,

$$
\begin{gathered}
\frac{r(t+\Delta t)-2 r(t)+r(t-\Delta t)}{\Delta t^{2}}=-\frac{1}{m} \frac{d V(r(t))}{d r} \\
r(t+\Delta t)=2 r(t)-r(t-\Delta t)-\Delta t \cdot \frac{1}{m} \cdot \frac{d V(r(t))}{d r}
\end{gathered}
$$

Hence, $r(t+\Delta t)$ can be found as long as $r(t)$ and $r(t-\Delta t)$ are known.

\subsection{Molecular Interactions}

The interactions between atoms are defined through potential $(U)$ which is a function of position of atoms. In full generality, three types of interactions are considered; i.e. bonded, Van der Waals, and Coulombic. The bonded interaction is divided into four catagories of bonds, angles, dihedrals, and impropers interactions.

$$
\begin{aligned}
& U_{\text {total }}=U_{\text {bonded }}+U_{v d W}+U_{\text {Coulomb }} \\
& U_{\text {bonded }}=U_{\text {Bonds }}+U_{\text {Angles }}+U_{\text {Dihedrals }}+U_{\text {Impropers }}
\end{aligned}
$$

The Van der Waals interactions $\left(U_{v d W}\right)$ refer to one type of potential between non-bonded atoms. In many simulations a simple interaction potential represents the essential fluid dynamics. The most commonly used force-field (potential and force-field terms are used interchangeably in this 
dissertation) is 12-6 Lennard-Jones potential.

$$
U_{l j}\left(r_{i j}\right)=4 \epsilon_{i j}\left[\left(\frac{\sigma_{i j}}{r_{i j}}\right)^{12}-\left(\frac{\sigma_{i j}}{r_{i j}}\right)^{6}\right]
$$

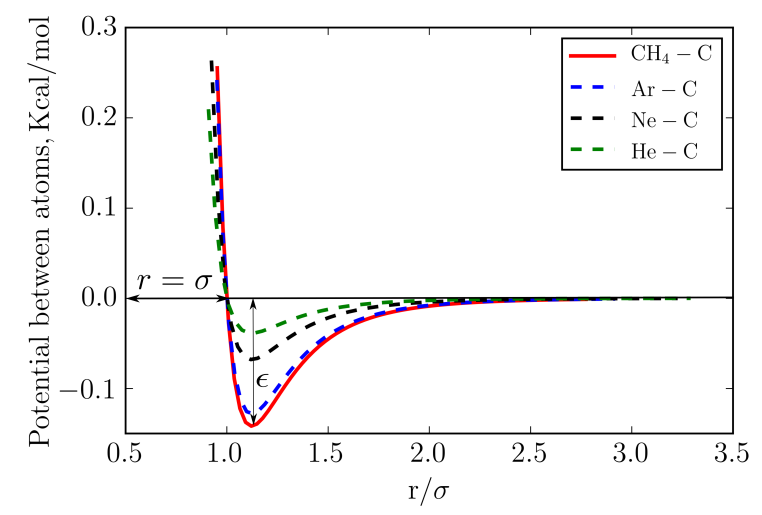

Figure 2.1: Lennard-Jones potential interactions of different gases with carbon in graphite as a function of dimensionless distance.

where $r_{i j}, \epsilon_{i j}$, and $\sigma_{i j}$ are separation distance, strength of interaction, and LJ well depth, respectively. Unlike interactions were determined using the Lorentz-Berthelot combining rules,

$$
\begin{gathered}
\sigma_{i j}=\frac{\sigma_{i i}+\sigma_{j j}}{2}, \\
\epsilon_{i j}=\sqrt{\epsilon_{i i} \epsilon_{j j}} .
\end{gathered}
$$

If electrostatic charges exist, the Coulombic interactions are considered.

$$
U_{\text {Coulomb }}\left(r_{i j}\right)=\frac{Q_{i} Q_{j}}{4 \pi \epsilon_{0} r_{i j}}
$$

where $Q_{i}$ and $Q_{j}$ are charges and $\epsilon_{0}$ is the permittivity of free space.

\subsubsection{OPLS-All Atom Force Field}

The optimized potentials for liquid simulations force field was developed by Jorgensen et al. [49]. The force field was parametrized for different molecules for the simulation of proteins and other 
organic liquids. The functional format of this force field is as follows,

$$
\begin{gathered}
U_{i j-\text { nonbonds }}=4 \epsilon_{i j}\left[\left(\frac{\sigma_{i j}}{r}\right)^{12}-\left(\frac{\sigma_{i j}}{r}\right)^{6}\right]+\frac{q_{i} q_{j}}{4 \pi \epsilon_{0} r} \\
U_{\text {bonds }}=\sum_{\text {bonds }} K_{r}\left(r-r_{0}\right)^{2} \\
U_{\text {angles }}=\sum_{\text {angles }} K_{\theta}\left(\theta-\theta_{0}\right)^{2}
\end{gathered}
$$

The value of parameters in above equations $\left(\epsilon_{i j}, \sigma_{i j}, q_{i}, q_{j}, K_{r}, r_{0}, K_{\theta}, \theta_{0}\right)$ for different molecules are listed in Jorgensen et al. [49]. In this dissertation, OPLS-All atoms force field is used to simulate the alkanes in organic nanochannels and nanocapillaries.

\subsubsection{COMPASS Force Field}

COMPASS (Condensed-phase Optimized Molecular Potentials for Atomistic Simulation Studies) force field parameterized against a wide range of experimental observables for organic compounds containing $\mathrm{H}, \mathrm{C}, \mathrm{N}, \mathrm{O}, \mathrm{S}, \mathrm{P}$, halogen atoms and ions and etc [98]. The force field presents the parameters needed to simulate the functional groups in organic compounds. The functional forms of this force field are as follow,

$$
\begin{gathered}
U_{v d W}=6.75 \epsilon_{i j}\left[\left(\frac{\sigma}{r}\right)^{9}-\left(\frac{\sigma}{r}\right)^{6}\right] \\
U_{q}=\frac{q_{i} q_{j}}{4 \pi \epsilon_{0} r} \\
U_{\text {bonds }}=\sum_{\text {bonds }}\left[k_{2}\left(r-r_{0}\right)^{2}+k_{3}\left(r-r_{0}\right)^{3}+k_{4}\left(r-r_{0}\right)^{4}\right] \\
U_{\text {angles }}=\sum_{\text {angles }}\left[k_{2}\left(\theta-\theta_{0}\right)^{2}+k_{3}\left(\theta-\theta_{0}\right)^{3}+k_{4}\left(\theta-\theta_{0}\right)^{4}\right]
\end{gathered}
$$

In simulation of kerogen structure, the COMPASS force field is employed because COMPASS force field is parametrized for different functional groups of a complex organic compounds. 


\subsection{Periodic Boundary Condition}

In molecular simulation, the number of atoms used in simulation is very small in compare to actual systems. Desktop computers can handle simulations with $10^{3}$ to $10^{6}$ atoms which is only a small portion of one mole of atoms $\left(N_{A}=6.022 \times 10^{2} 3\right)$. The behavior of simulation is different by a large number of surrounding atoms (in the order of $10^{2} 3$ ) which cannot be considered in simulations explicitly [14]. The influence of surrounding atoms can be implicitly included in simulations by handling of boundary conditions. The periodic boundary conditions help to simulate the infinite systems using finite size simulation cells without increasing computational costs.

\section{$2.4 \quad$ Statistical Ensembles}

The ensemble is one of the main concepts in statistical mechanics. Consider that a given molecular system is replicated many times while the macroscopic properties (temperature, density, number of atoms and etc.) of each of these replicas are the same. The position and velocity of these replicas (which are called microstate) does not need to be the same since we are interested in macroscopic properties of the system. An ensemble is the probability distribution of microstates for a set of conditions (macrostate). The actual value of bulk properties of a system should be determined by averaging over the ensemble. In molecular dynamics, true thermodynamics properties of a system is determined by averaging over successive configurations by implicitly assuming that the ensemble average is the same as average over time of one replica of the system. This assumption is known as Ergodic Hypothesis [54]. 


\section{Chapter 3}

\section{Adsorption and Transport of Gases in Simplified Kerogen Model}

In modeling of fluid transport in organic nanopores of shale, particular attention should be paid to the gas-wall interactions, specifically the adsorption phenomena, and the fact that the size of pores are comparable with the mean-free-path of the gas molecules. The objective for this work is to investigate the significance of the adsorbed gas molecules in the total mass flux of organic nano-scale channels.

Molecular Dynamics (MD) is proved to be a credible technique to examine dynamics of atomic-level phenomena. In this study, transport of four different gases, methane and argon (high adsorption affinity) and helium and neon (low adsorption affinity), is studied and their velocity and mass flux profiles are analysed using dual control volume grand canonical molecular dynamics (DCV-GCMD) simulations. DCV-GCMD simulations are performed for different pressures, pressure gradients, and channel sizes. Computed normalized velocities are close to one for all the gases and channel heights, which shows the the velocity profiles are plug-shaped. For all the gases, as the pressure increases, the density and normalized velocity of the molecules at the wall increase. Furthermore, as pressure increases, velocity profiles change from a plug-liked velocity profiles to parabolic profiles because the viscosity of the fluids increase as the pressure increases. The contribution of the adsorbed gas to the total mass flux across the channel for argon and methane could be more than $50 \%$. This contribution deceases as pressure in the channel increases. Investigation of the effect of the channel 
length on the velocity profiles shows that the channel length have a significant impact on transport of gases through nanochannels. For channels with height-length ratio less than 10, the results can be masked by the boundary effects.

Assuming an ideal vapor phase, the transport equation is then written in the form:

$$
J=-D \frac{d c}{d z}, \quad D=D_{0} \frac{d \ln (p)}{d \ln (c)}
$$

where, $D$ is the Fickian transport diffusivity, $D_{0}$ is referred to as corrected diffusivity and $\frac{d \ln (p)}{d \ln (c)}$ as thermodynamic factor. If the system is thermodynamically ideal, $\frac{d \ln (p)}{d \ln (c)} \rightarrow 1.0$, then the Fickian and corrected diffusivities become identical. However, in the more general case of a thermodynamically nonideal system, the Fickian transport diffusivity is seen to be the product of the corrected diffusivity and the thermodynamic correction factor. Beyond the Henry's law region the simple Langmuir model is often used to represent the behavior of adsorption systems in an approximate way. For a single adsorbed component [51]:

$$
\theta=\frac{c}{c_{s}}=\frac{b p}{1+b p}, \quad \frac{d \ln (p)}{d \ln (c)}=\frac{1}{1-c / c_{s}}=\frac{1}{1-\theta}
$$

where $\theta$ is referred to as the fractional loading and $b$ is the adsorption equilibrium constant.

Flow and diffusion of gas molecules in microporous systems have been extensively studied using three different molecular dynamics simulation methods: Equilibrium Molecular Dynamics (EMD), external field Non-Equilibrium Molecular Dynamics (NEMD), and boundary driven non-equilibrium molecular dynamics (dual control volume grand canonical molecular dynamics or DCV-GCMD) [4].

In NEMD simulation method, an external force is applied to gas molecules. The advantages of NEMD simulation method is that it is computationally efficient and easy to implement. The drawback of the method is the conversion of the external force to equivalent chemical potential gradient. The boundary driven non-equilibrium molecular dynamics method allows the gas transport between two reservoirs with fixed chemical potentials. The chemical potential of the two reservoirs are kept constant by insertion and deletion of gas molecules (usually performed through a grand canonical Monte Carlo or GCMC procedure) and the transport through the medium of interest is performed by molecular dynamics. Insertion and deletion of molecules is the significant fraction of total simulation cost in this method.

NEMD simulations have been applied to investigate the slippage of a non-adsorbing gas (helium) 
[32]. The results indicated that the experimental measured permeabilities are two order of magnitude larger than NEMD results. Flow of methane in a $5 \mathrm{~nm}$ tube has been performed using NEMD simulations by [83]. They showed that the adsorbed phase transport is different from slippage of non-adsorbing gases and concluded that the formulations that is used for non-adsorbing gases, may not predict the transport of adsorbing gases. Collell et al. [25] performed molecular dynamics simulations of hydrocarbons flowing through a molecular model of kerogen type II. They showed that the flow inside kerogen is of diffusive nature. He et al. [37] performed NEMD simulations to study the transport behavior of natural gas (methane) in the reconstructed nano-scale digital rock. They selected a representative kerogen molecule based on thermal maturity and the origin. They found that as the molar density increases, the transport diffusivity increases. Dual control volume grand canonical molecular dynamics (DCV-GCMD) simulations have been used to investigate the sensitivity of transport properties of methane, carbon dioxide, and nitrogen to pore size, porosity, and pressure gradient in 3-D carbon-based systems representative of porous carbon-based materials [33]. Jin and Firoozabadi [47] investigated flow of methane in carbon nanotubes using DCV-GCMD simulations. The mobility of adsorbed phase in high and low pressures were demonstrated. Feng and Akkutlu [30] performed NEMD and DCV-GCMD simulations to study steady-state flow of methane and of methane-butane mixture in nano-scale carbon capillaries. They observed that fluid flow velocity and mass flux are significant near the capillary surfaces where adsorption takes place. Moreover, they showed that the transport behavior is additionally controlled by the inner surface morphology (surface roughness).

\subsection{DCV-GCMD Simulations in Organic Nanochannels}

The objective of this work are to compare the transport of gases with high adsorbing capacities (methane and argon) and low adsorption capacity gases (helium and neon) in similar simulation setups of graphite nanochannels. To represent the flow of gases in organic pores, the geometries are simplified to single smooth carbon channels to isolate the discussion of diffusion mechanisms from the effects of geometry and pore size distribution. Monte Carlo simulation are performed within Grand Canonical ensemble to predict the adsorption properties (excess and absolute). DCV-GCMD simulations are then carried out in 2 and $5 \mathrm{~nm}$ graphite nanochannels at different pressures and pressure gradients. Effect of system geometry (channel height and length), average pore pressure, and pressure gradient on transport of gases in graphite channels are also investigated. Furthermore, 
contribution of the adsorbed phase transport on the total transport in the channels are investigated.

\subsubsection{Computational Methodology}

Problem Setup A single graphite nanochannel is created using Moltemplate molecular builder software [46]. Each side of nanochannel is consisted of three graphite layers with a distance of 0.335 nm. The distance between two adjacent carbon atoms in the same graphite layer is 0.142.

The nanochannel is placed between two cubic bulk reservoirs at different pressures and at $300 \mathrm{~K}$ to perform DCV-GCMD simulations, Fig. 3.1. Since a periodic boundary condition is considered in flow direction, connecting the low and high reservoirs could cause high fluctuations in the pressures in the reservoirs. Therefore, two graphite walls are placed at the end of each reservoir to avoid their direct contact. The length of the left $(\mathrm{H})$ (downstream) and right (L) reservoirs (upstream) are chosen to be $2 \mathrm{~nm}$ and $10 \mathrm{~nm}$ for simulation with channel heights $2 \mathrm{~nm}$ and $5 \mathrm{~nm}$, respectively.

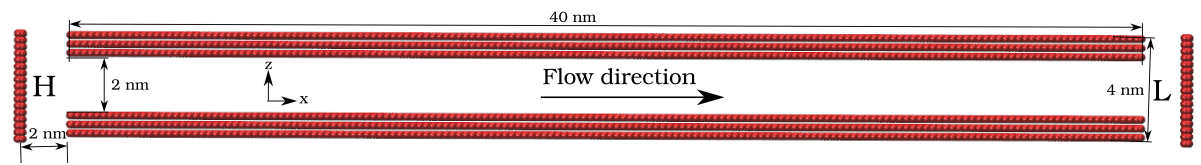

Figure 3.1: Simulation setup for the $2 \mathrm{~nm}$ channel. The channel length is $40 \mathrm{~nm}$. "H" and " $\mathrm{H}$ " represent the high and low pressure reservoirs, respectively.

The TraPPE model [69] is used to simulate the gases (methane, argon, helium, and neon). The charges of carbon and hydrogen atoms are not considered. Lennard-Jones (LJ) pairwise additive potential field was employed to represent the gas molecules interaction as,

$$
U_{l j}\left(r_{i j}\right)=4 \epsilon_{i j}\left[\left(\frac{\sigma_{i j}}{r_{i j}}\right)^{12}-\left(\frac{\sigma_{i j}}{r_{i j}}\right)^{6}\right]
$$

where $r_{i j}, \epsilon_{i j}$, and $\sigma_{i j}$ are separation distance, strength of interaction, and LJ well depth, respectively. Unlike interactions were determined using the Lorentz-Berthelot combining rules,

$$
\begin{gathered}
\sigma_{i j}=\frac{\sigma_{i i}+\sigma_{j j}}{2}, \\
\epsilon_{i j}=\sqrt{\epsilon_{i i} \epsilon_{j j}} .
\end{gathered}
$$

The separation distance $(\sigma)$ and strength $(\epsilon)$ parameters for all gases are shown in Table 3.1. A 
cut-off distance of $10 \mathrm{~A}$ is considered for all simulations.

Table 3.1: Lennard-Jones parameters

\begin{tabular}{c|c|c|l} 
Interaction & $\epsilon / k_{B}(K)$ & $\sigma(A)$ & Reference \\
\hline \hline $\mathrm{CH}_{4}-\mathrm{CH}_{4}$ & 148 & 3.73 & {$[74]$} \\
\hline $\mathrm{C}-\mathrm{C}$ & 34.45 & 3.407 & {$[89]$} \\
\hline $\mathrm{Ne}-\mathrm{Ne}$ & 36.83 & 2.789 & {$[75]$} \\
\hline $\mathrm{Ar}-\mathrm{Ar}$ & 119.8 & 3.405 & {$[6]$} \\
\hline $\mathrm{He}-\mathrm{He}$ & 10.9 & 2.64 & {$[75]$}
\end{tabular}

Simulation Method In order to simulate flow of gases in the graphite channel, DCV-GCMD method $[38,110]$ is employed. The MD simulation time integration of equation of motion are performed using Verlet velocity algorithm with a time step of $3 \mathrm{fs}$ (femtoseconds). The pressure inside the control volumes is kept constant using adequate number of GCMC insertion and deletion. The inserted molecules are assigned based on specified reservoir temperature $(300 \mathrm{~K})$. The driving force for gas molecules movement were the pressure or chemical potential difference between the two control volumes ( $\mathrm{L}$ and $\mathrm{H}$ ). The wall and fluid temperatures were kept constant at $300 \mathrm{~K}$ in $\mathrm{NVT}$ (constant number of molecules, constant volume and constant temperature) ensemble. All the simulations are performed using Large-scale Atomic/Molecular Massively Parallel Simulator (LAMMPS) (Plimpton, 1995). Visual Molecular Dynamics (VMD) (Humphrey et al., 1996) is used for visualization. All the simulations are performed using Large-scale Atomic/Molecular Massively Parallel Simulator (LAMMPS) [81] and Visual Molecular Dynamics (VMD) [43] is used for visualization.

\subsubsection{Results and Discussion}

\section{Adsorption Isotherms}

GCMC simulations are performed to determine the adsorption isotherms of neon, helium, argon, and methane in 2 and $5 \mathrm{~nm}$ channels for a pressure range of 10 to 330 atmosphere and a constant temperature of $300 \mathrm{~K}$. In order to study adsorption behavior of gases under confinements, two quantities are usually defined as follows. Absolute adsorption is the quantity of the gas in the adsorbed state. Excess adsorption is the additional gas adsorbed amount per unit volume compared 
with the amount in the same volume in the absence of pore walls. Calculations of adsorption loadings are performed based on the multilayer adsorption of gases to the pore surfaces (Figs. 3.2 and 3.3).

Absolute adsorption quantities increase as the pressure increases for both channel heights. The absolute adsorption isotherms of argon and methane are similar in both channel; for argon and methane in the $5 \mathrm{~nm}$ channel, the isotherms are overlapping and reach to $15 \mathrm{mmol} / \mathrm{cm}^{3}$ at $330 \mathrm{~atm}$. However, for both argon and methane, the absolute adsorption is slightly higher in the $2 \mathrm{~nm}$ channel compared to the $5 \mathrm{~nm}$ channel. The absolute loadings of neon and helium are smaller and reach to approximately $10.5 \mathrm{mmol} / \mathrm{cm}^{3}$ for both channel sizes. Absolute adsorption of neon is slightly higher than that of helium in $5 \mathrm{~nm}$ channel. The results show that helium and neon have the lower adsorption capacities compared to methane and argon.

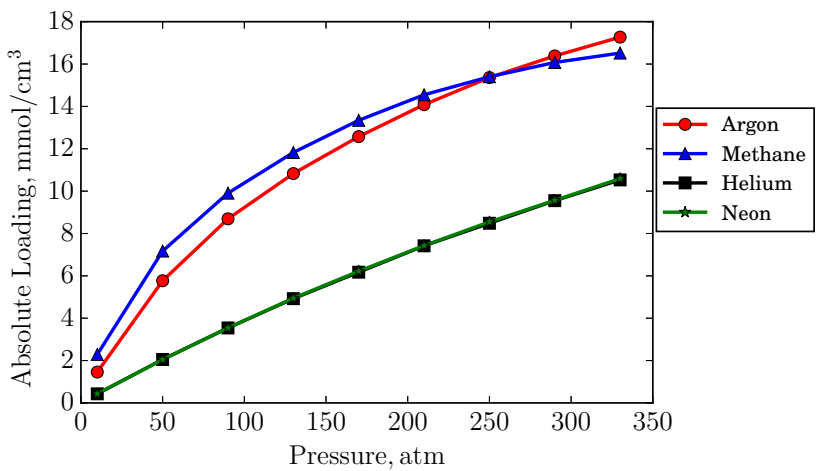

(a) $2 \mathrm{~nm}$ channel

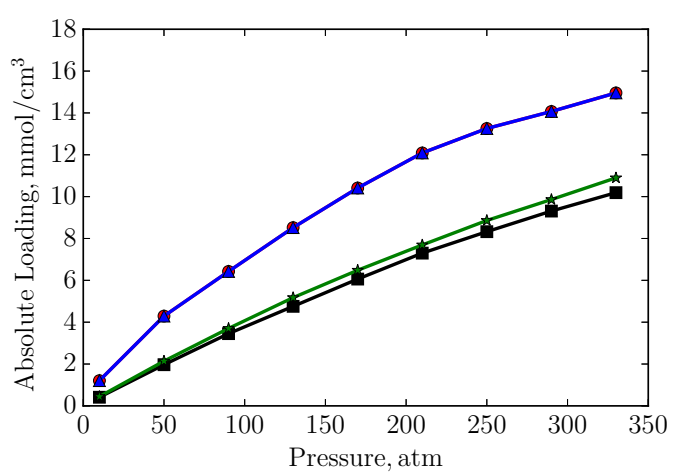

(b) $5 \mathrm{~nm}$ channel

Figure 3.2: Absolute adsorption isotherms of argon, methane, helium, and neon for 2 and $5 \mathrm{~nm}$ channel heights.

As pressure increases, excess loadings increase to reach a maximum value at an optimum pressure and then decrease (Figs. 3.3). For both channel sizes, methane has a smaller value of optimum pressure than argon. The maximum excess adsorption of argon and methane are 5.1 and $5.6 \mathrm{mmol} / \mathrm{cm}^{3}$ in the $2 \mathrm{~nm}$ channel, respectively. These values decrease to 2 and $2.1 \mathrm{mmol} / \mathrm{cm}^{3}$ for argon and methane in the $5 \mathrm{~nm}$ channel, respectively. The excess adsorption for neon and helium slightly increase followed by a decline. Neon adsorption loadings decrease from 0.025 to $-0.904 \mathrm{mmol} / \mathrm{cm}^{3}$ in the $2 \mathrm{~nm}$ channel and from 0.042 to $-0.598 \mathrm{mmol} / \mathrm{cm}^{3}$ in the $5 \mathrm{~nm}$ channel. Helium adsorption loadings decrease from 0.027 to $-1.092 \mathrm{mmol} / \mathrm{cm}^{3}$ in the $2 \mathrm{~nm}$ channel and from 0.010 to -1.432 $\mathrm{mmol} / \mathrm{cm}^{3}$ in the $5 \mathrm{~nm}$ channel. Similar to absolute adsorption results, excess values demonstrate that the neon and helium gases are less-adsorbing compared with argon and methane. 


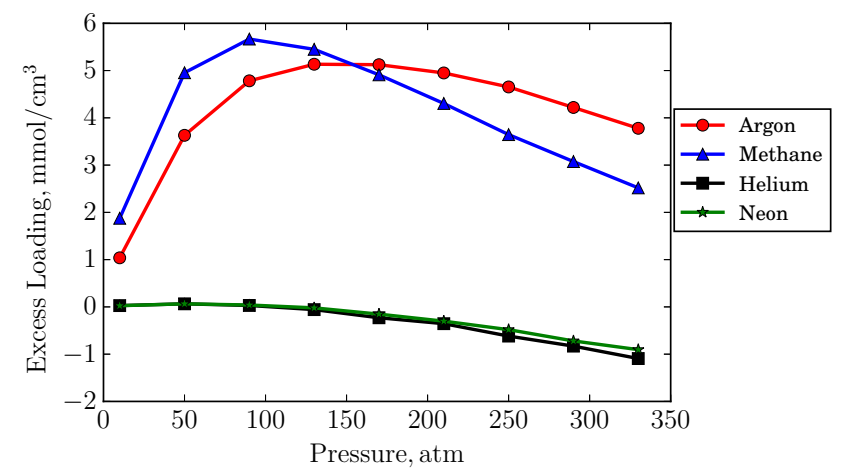

(a) $2 \mathrm{~nm}$ channel

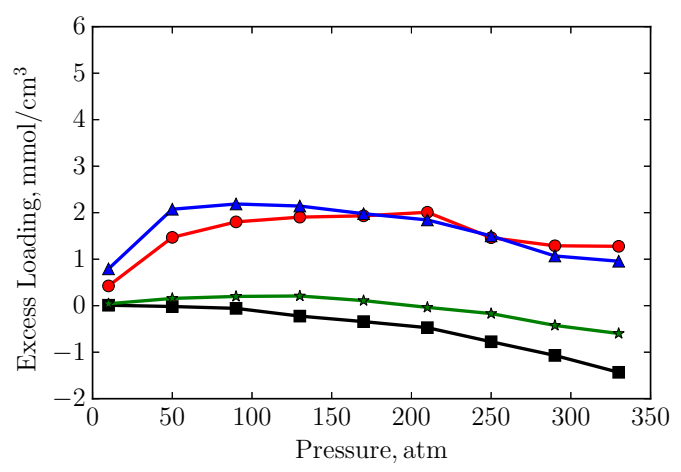

(b) $5 \mathrm{~nm}$ channel

Figure 3.3: Excess adsorption isotherms of argon, methane, helium, and neon in 2 and $5 \mathrm{~nm}$ channel heights.

\subsubsection{Chemical Potential Driven Flow}

In this section, the velocity and mass flux profiles of methane, argon, neon, and helium in 2 and 5 $n m$ channels are presented. Simulations are performed at different gas pressures and pressure drops across the channels. The channel height is divided into equally-sized bins, each with a height of 0.06 angstroms.

The computed velocity profiles are normalized with the average velocity values across the channel height. The normalized velocities at the same average pressure are approximately the same for different pressure drops for linear response regime [4]. Average velocities of argon molecules across the $2 \mathrm{~nm}$ channel are plotted against the pressure gradient in Fig. 4 . These results show that the simulations are in linear response regime.

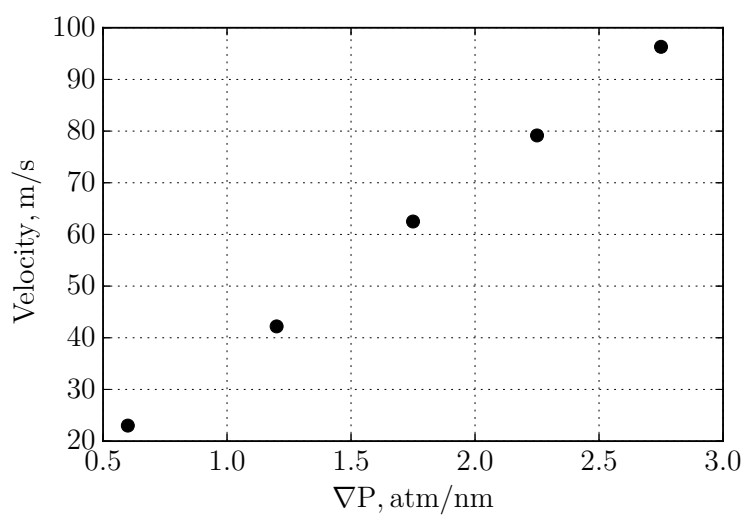

Figure 3.4: Average velocities across the channel height vs. pressure gradient. Simulations are performed in the linear response regime. 


\section{A. 2 nm channel}

MD simulations are performed at three different average pressures of 20, 50, and $90 \mathrm{~atm}$. These pressures are the average pressure of the low and high pressure reservoirs. The normalized velocity profiles along with the corresponding density profiles are presented for argon and methane in Fig. 3.6 and for neon and helium in Fig. 3.7.

Density molecular weight ratios of the adsorbed argon molecules increase from 18.5 to $55 \mathrm{mmole} / \mathrm{cm}^{3}$ as pressure increases from 20 to 90 atm (Fig. 3.6a). These ratios increase from 33.87 to 68.56 $\mathrm{mmole} / \mathrm{cm}^{3}$ for methane (Fig. 3.6b). As pressure increases, there is slight increase in the density of the molecules in the second layer for methane and argon. The formation of the second layer is more pronounced in $90 \mathrm{~atm}$ than in $50 \mathrm{~atm}$.

Density molecular weight ratios of the adsorbed neon molecules increase from 2.97 to $12.39 \mathrm{mmole} / \mathrm{cm}^{3}$ as pressure increases from 20 to $90 \mathrm{~atm}$ (Fig. 3.7b). These ratios increase from 3.89 to 10.77 $\mathrm{mmole} / \mathrm{cm}^{3}$ for helium (Fig. 3.7a). At this pressure range, pressure does not reach the saturation pressure (the pressure beyond which the adsorbed phase density does not significantly change with pressure). Density molecular weight ratios of the adsorbed helium and neon layers at $90 \mathrm{~atm}$ are 2.08 and 3.34 times larger than its average value in the channel. these values reach 6.7 and 7.14 for argon and methane, respectively.

The adsorption loadings (or density molecular weight ratios) are the reflection of strength of potential between the gas molecules and the molecules of the organic channel (Fig. 5). The strength of potential between the methane and graphite molecules is highest compared to other gases and therefore, its adsorption loadings on the channel walls are higher as well. The strength of potential of argon, neon, and helium have lower values, respectively, and their adsorption amount have the same trend.

The normalized velocity profiles range from 0.75 to 1.2 for argon (Fig. 3.6a) and 0.75 to 1.3 for methane (Fig. 3.6b), respectively. For both argon and methane, as the pressure increases, the normalized velocity of the molecules at the wall increases. This may be due to the fact that viscosities of fluids are a function of the Knudsen number (and thus, the pressure). Viscosity of the gas decreases as Knudsen number increases (or as pressure decreases) [53, 72, 84]. Moreover, as pressure increases, ratio of the normalized velocity of the gas molecules at the center of channel to the normalized velocity of the first gas layers in the vicinity of channel wall (slip velocity) becomes smaller. This is 


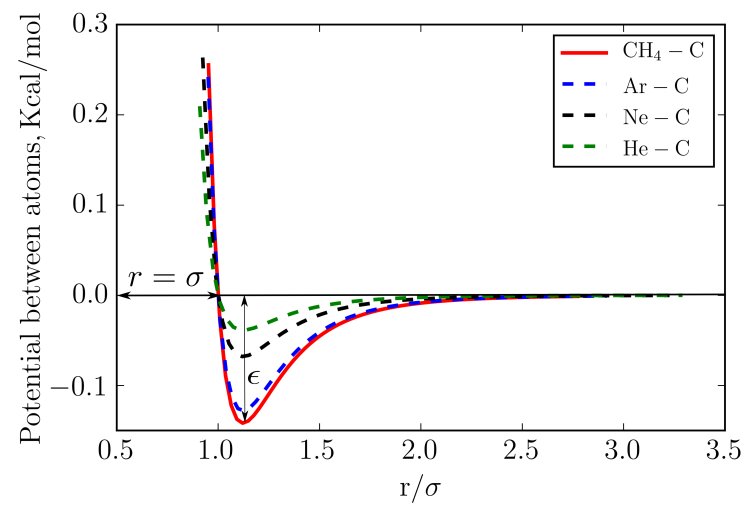

Figure 3.5: Lennard-Jones interaction between different gases and organic channel.

contrary to the published results on the theory of slip in channels [53]. According to these theories, the normalized velocity of the adsorbed gas molecules on the channel wall becomes larger as pressure increases (i.e., lower Knudsen number).

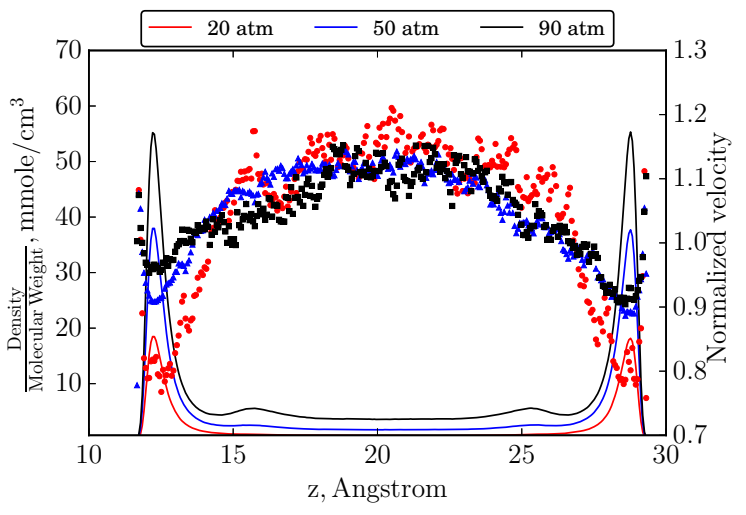

(a) Argon

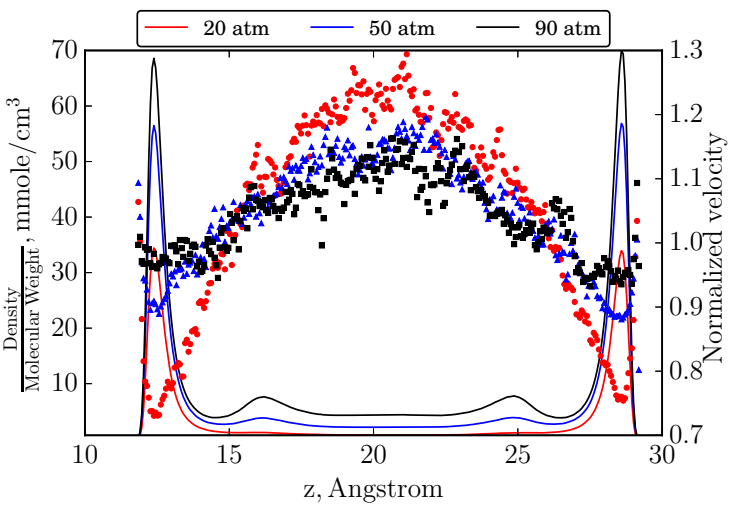

(b) Methane

Figure 3.6: Density and normalized velocity profiles of argon and methane in the $2 \mathrm{~nm}$ channel.

Normalized velocity profiles of neon and helium are approximately flat (the normalized velocities are close to one). Normalized velocity values of the first layers of gas molecules at the channel wall depend on the strength of the force fields exerted to the gas molecules by the solid molecules of the wall. The higher the attractive forces, the harder it will be for the gas molecules to escape from the the force field of the solid molecules at the wall and the lower the values of the normalized velocities at the channel wall will be. For methane and argon (adsorbing gases), the normalized velocity of the gas molecules in the first layer in vicinity of the channel walls are smaller than those for neon and helium (less adsorbing gases). Methane and argon have higher strength of potential compared to helium and neon, which causes the molecules in vicinity of the wall have slower velocities. 


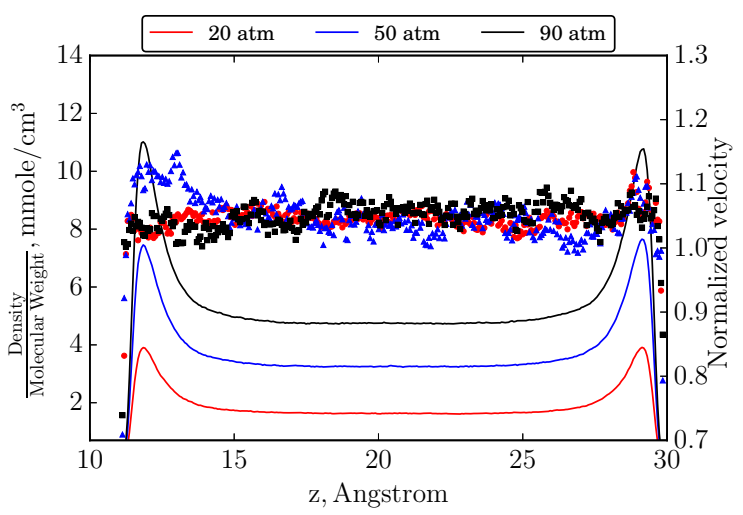

(a) Helium

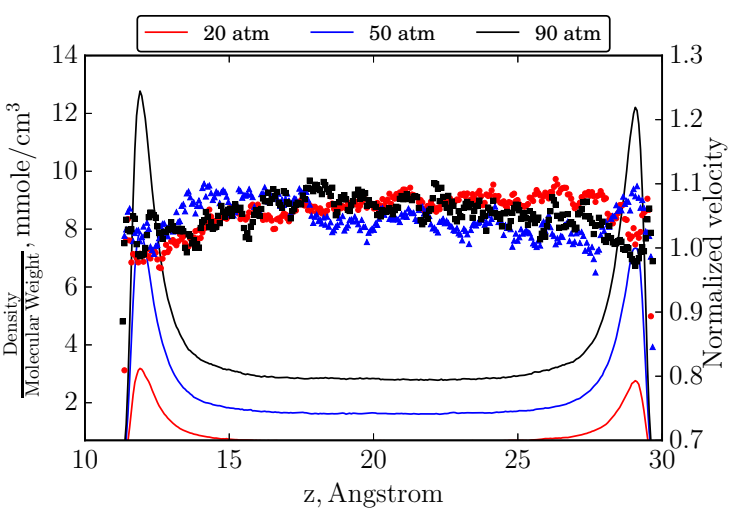

(b) Neon

Figure 3.7: Density and normalized velocity profiles of helium and neon in the $2 \mathrm{~nm}$ channel.

\section{B. 5 nm channel}

Density and normalized velocity values of the gas molecules in the $5 \mathrm{~nm}$ height channel are investigated for argon and methane in Fig. 3.8 and for helium and neon in Fig. 3.9.

Density molecular weight ratios of the adsorbed argon molecules increase from 21.4 to $57.1 \mathrm{mmole} / \mathrm{cm}^{3}$ as pressure increases from 20 to $90 \mathrm{~atm}$ (Fig. 3.8a), which are similar to those for $2 \mathrm{~nm}$ channel (Fig. 3.6a). These values increases from 32.5 to $62.1 \mathrm{mmole} / \mathrm{cm}^{3}$ for methane as pressure increases as pressure increases from 15 to $60 \mathrm{~atm}$ (Fig. 3.8b). The formation of the second adsorbed layer of argon and methane molecules in the channel is also evident for $5 \mathrm{~nm}$ channel. Density molecular weight ratios of the adsorbed argon and methane layers 10.7 and 12.9 times larger than the average density of the channel for pressures 90 and 60 atm, respectively.

Density molecular weight ratios of the adsorbed helium molecules increase from 1.75 to $5.8 \mathrm{mmole} / \mathrm{cm}^{3}$ as pressure increases from 50 to $180 \mathrm{~atm}$ (Fig. 3.9a). These ratios increase from 3.45 to $9 \mathrm{mmole} / \mathrm{cm}^{3}$ for neon as pressure increases from 20 to $60 \mathrm{~atm}$ (Fig. 3.9b). Density molecular weight ratios of the adsorbed helium and neon layers are smaller than those for argon and methane.

Similar to the flow in $2 \mathrm{~nm}$ channel, the normalized velocity profiles for argon and methane are plug-shaped. The normalized velocity profiles range from 0.7 to 1.15 for argon (Fig. 3.8a) and 0.6 to 1.3 for methane (Fig. 3.8b). As pressure increases, normalized velocity values of the adsorbed argon and methane molecules (first layers of argon and methane molecules next to the channel wall) increase. The difference between the normalized velocity values of first and second gas layers 
decrease as pressure increases. Furthermore, similar to the $2 \mathrm{~nm}$ channel, as pressure increases, the range of the normalized velocity in the channel wall becomes smaller.

Normalized velocity profiles of neon and helium are approximately flat (the normalized velocities are close to one) (Fig. 3.9), which is similar to those in the $2 \mathrm{~nm}$ channel. These velocity profiles are different from the prediction made by current models $[10,55]$.

The results of this section confirms that the gas transport in organic microporous materials is enhanced [95] compared with other media. Furthermore, to have a further understanding of the gas transport in organic matters of shale, tortuosity and presence of other functional groups should be considered. Moreover, effect of the viscosity change as a function of Knudsen number should be included in the transport models at slip and transition flow regimes.

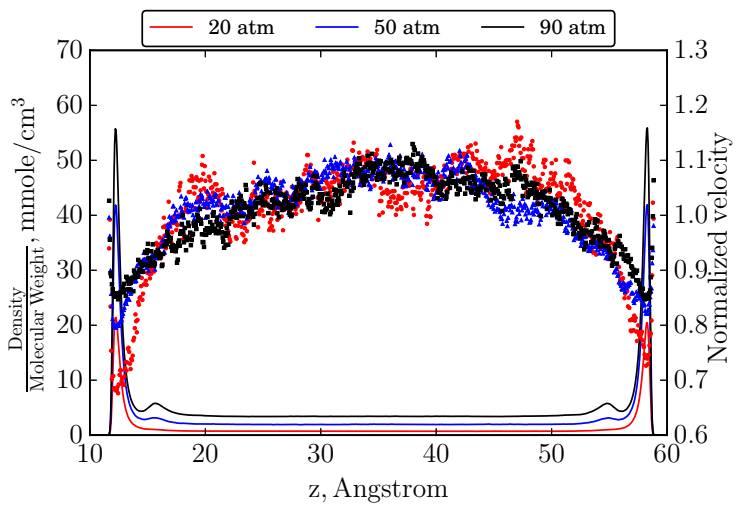

(a) Argon

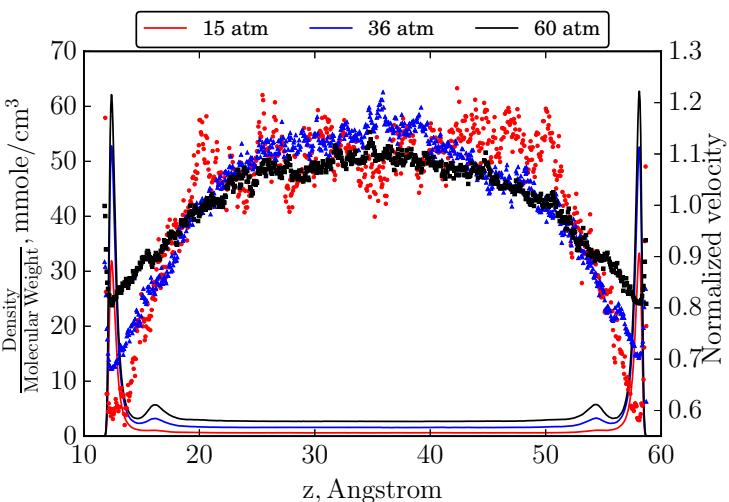

(b) Methane

Figure 3.8: Density and normalized velocity profiles of argon and methane in the $5 \mathrm{~nm}$ channel.

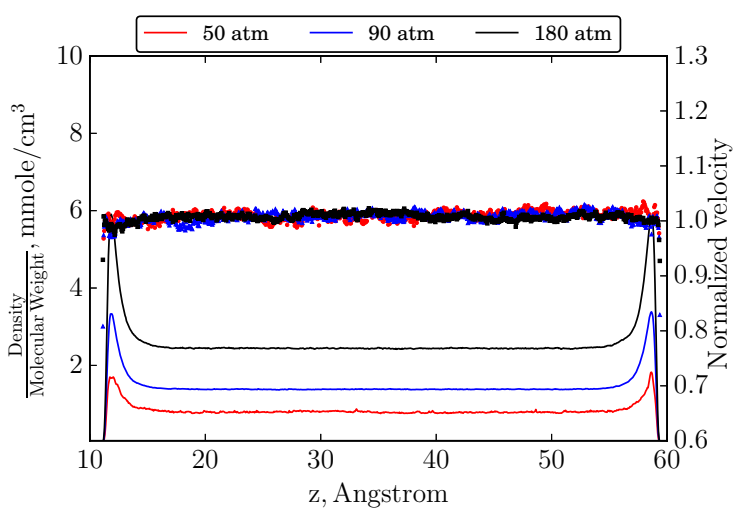

(a) Helium

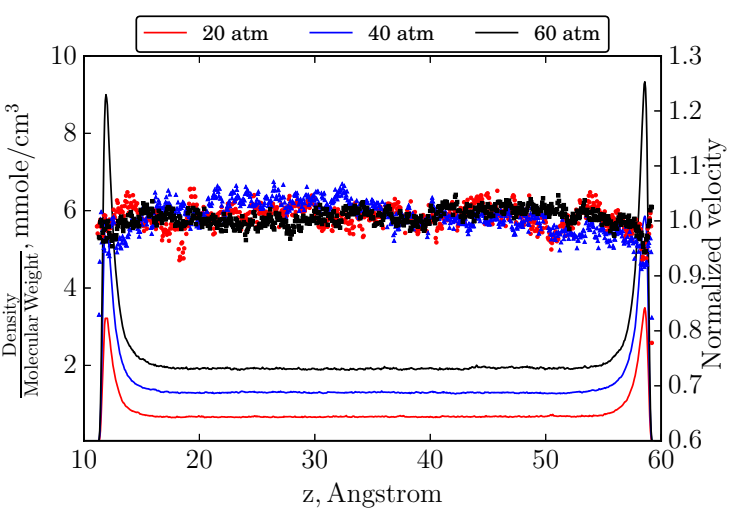

(b) Neon

Figure 3.9: Density and normalized velocity and profiles of helium and neon in the $5 \mathrm{~nm}$ channel. 


\section{Mass Flux Profiles}

To compare the mass flux profiles of different gases in the channels, computed mass flux values across the channel are non-dimensionlized; Average velocity of each bin in the channel is multiplied by the average density at the bin and divided by total mass flux through the channel. As expected, the largest dimensionless mass flux values correspond to the bins at the first adsorbed molecule layers for argon and methane (Fig. 3.10). This is due to the fact that the density of argon and methane is higher than its density at bulk phase and the velocity profiles are relatively plug-shaped.

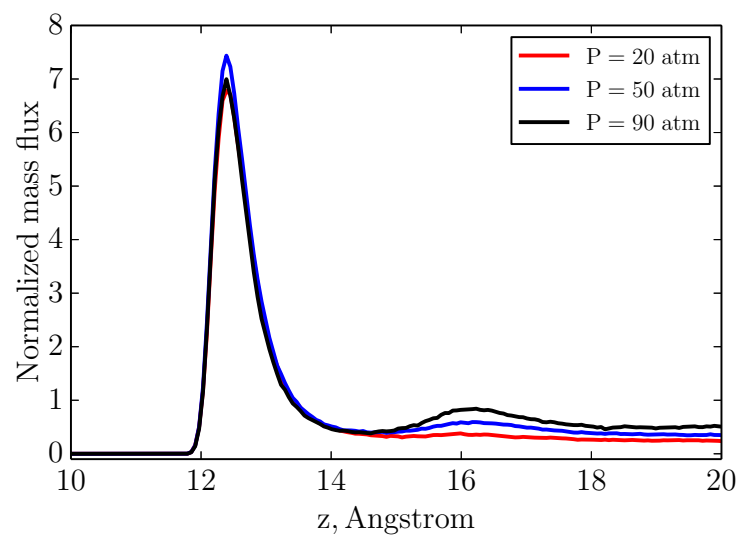

(a) $2 \mathrm{~nm}$ channel

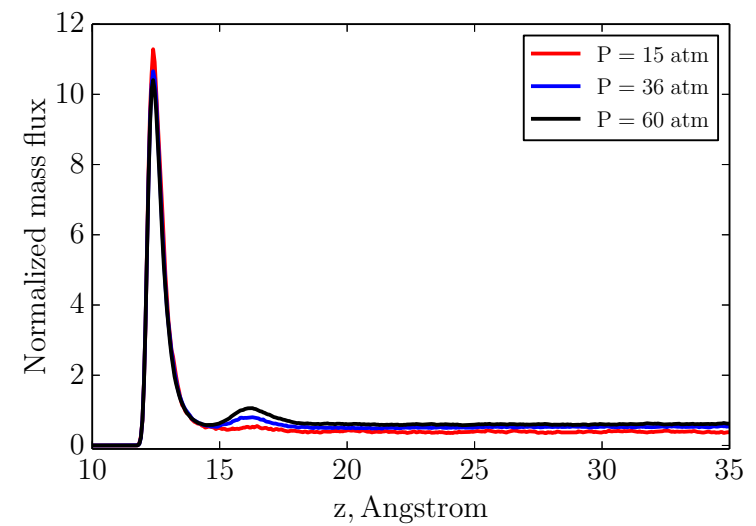

(b) $5 \mathrm{~nm}$ channel

Figure 3.10: Dimensionless mass flux profile of methane in the 2 and $5 \mathrm{~nm}$ channels. The profiles are plotted for the channel half height.

\section{Diffusion Coefficients}

For all the gases in this study, transport or Fickian diffusion coefficients are calculated at different pressures. Three simulation with different pressure gradients are run at each pressure and average transport diffusivity values are calculated. For each channel size and gas type, average transport diffusivity coefficients and standard deviations are listed in (Table 3.2).

Fickian constitutive relation describes the transport of single-component fluids as,

$$
J=-D_{t}(c) \nabla c
$$

where $D_{t}$ is the transport diffusivity, $\nabla c$ is the spatial concentration gradient, and $J$ is the molecular 
diffusive flux. This equation can be written in the terms of pressure gradient as [86],

$$
J=-\frac{M D_{t}}{R T} \nabla p
$$

where $M$ is the molar mass, $T$ is temperature, and $R$ is the universal gas constant.

The computed transport diffusivities for the channels are relatively independent of the gas pressure in the channel. Although for some of the gases in the channels, reductions or increase in the values can be observed, but these changes are not significant. However, a larger dataset of diffusion coefficients are needed to determine if the changes are statistically significant. The computed values for the 2 $\mathrm{nm}$ channel are smaller than those in the $5 \mathrm{~nm}$ channel for argon and methane. The same trend is also observed for neon. Transport diffusivity coefficients of less-adsorbing gases (helium and neon) do not change significantly as pressure changes in $5 \mathrm{~nm}$ channel.

\subsubsection{Effect of Channel Length}

In the simulations of the gas transport in straight channels, the channel should be sufficiently long to exclude the boundary effects in the axial directions [26]. Distance between the high and low pressure reservoirs have been reported to impact the gas flow [47]. In order to investigate the effect of the channel length on the results, simulations are performed in $2 \mathrm{~nm}$ channel using methane in channels with length 10,20, and $40 \mathrm{~nm}$ corresponding to width to length ratio of 5, 10, and 20, respectively. Velocity profiles of methane molecules are plotted at the same pressure gradient of $14.6 \mathrm{psi} / \mathrm{nm}$ in

Fig. 3.11. Based on the results, velocity values at the $10 \mathrm{~nm}$-long channel (the shortest channel) are the lowest. The velocity values of the first gas layers at the vicinity of the walls and also those at the center of the channel increase as the width to length ratio increase. The velocity profiles at the ratios of 10 and 20 are close to one another. Therefore, the simulation studies performed with small channel width to length ratios, may not be representative of the realistic transport in the organic nano-scale channels. 
Table 3.2: Transport diffusion coefficients for methane, argon, helium, and neon for all the channel sizes.

\begin{tabular}{|c|c|c|c|c|}
\hline Gas & $\begin{array}{c}\text { Channel Height } \\
(\mathrm{nm})\end{array}$ & $\begin{array}{c}\text { Pressure } \\
(\mathrm{atm})\end{array}$ & $\begin{array}{l}\text { Diffusion Coefficient } \\
\qquad\left(10^{-7} \mathrm{~cm}^{2} / \mathrm{s}\right)\end{array}$ & $\begin{array}{l}\text { Standard Deviation } \\
\qquad\left(10^{-7} \mathrm{~cm}^{2} / \mathrm{s}\right)\end{array}$ \\
\hline \multirow{6}{*}{ Methane } & \multirow{3}{*}{2} & 20 & 4.11 & 1.58 \\
\hline & & 50 & 3.35 & 0.43 \\
\hline & & 90 & 2.55 & 0.72 \\
\hline & \multirow{3}{*}{5} & 15 & 6.23 & 0.75 \\
\hline & & 36 & 6.30 & 0.29 \\
\hline & & 60 & 7.07 & 0.08 \\
\hline \multirow{6}{*}{ Argon } & \multirow{3}{*}{2} & 20 & 6.32 & 0.27 \\
\hline & & 50 & 5.78 & 1.18 \\
\hline & & 90 & 5.76 & 1.37 \\
\hline & \multirow{3}{*}{5} & 20 & 11.2 & 1.89 \\
\hline & & 50 & 11.9 & 0.54 \\
\hline & & 90 & 13.3 & 0.08 \\
\hline \multirow{6}{*}{ Helium } & \multirow{3}{*}{2} & 20 & 4.00 & 0.91 \\
\hline & & 50 & 2.01 & 0.73 \\
\hline & & 90 & 1.63 & 0.75 \\
\hline & \multirow{3}{*}{5} & 50 & 2.55 & 0.43 \\
\hline & & 90 & 2.47 & 0.49 \\
\hline & & 180 & 2.38 & 0.33 \\
\hline \multirow{6}{*}{ Neon } & \multirow{3}{*}{2} & 20 & 4.39 & 0.08 \\
\hline & & 50 & 3.58 & 0.15 \\
\hline & & 90 & 4.66 & 2.13 \\
\hline & \multirow{3}{*}{5} & 20 & 10.9 & 1.21 \\
\hline & & 40 & 10.9 & 0.75 \\
\hline & & 60 & 11.5 & 1.05 \\
\hline
\end{tabular}




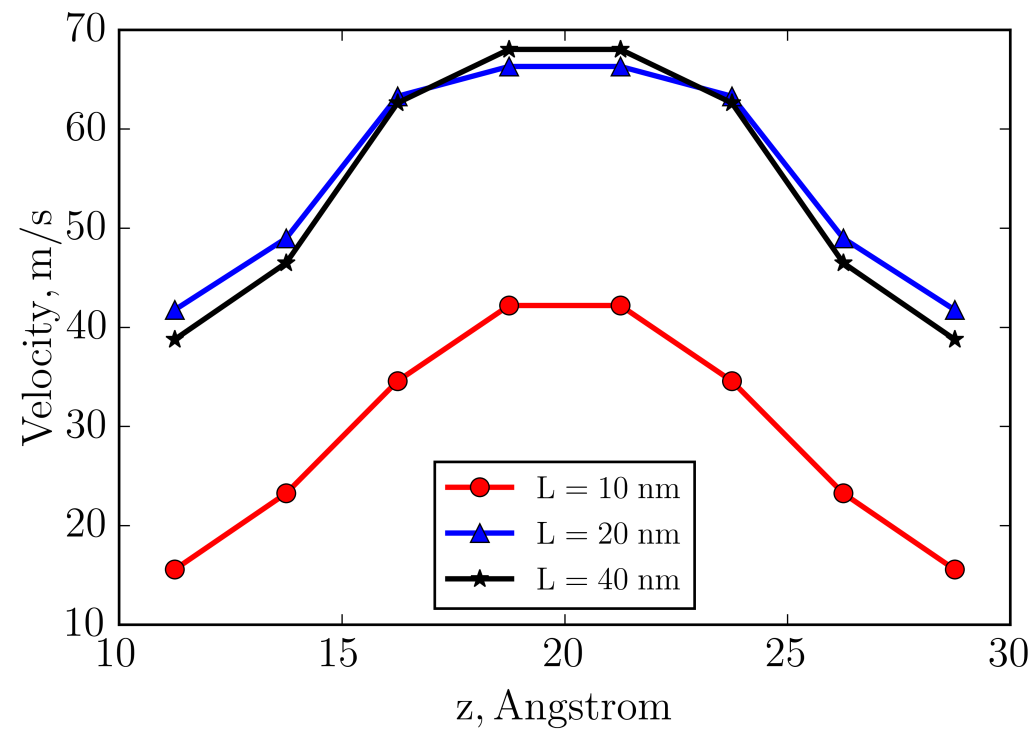

Figure 3.11: Sensitivity of the velocity of methane molecules to channel length for a $2 \mathrm{~nm}$ channel.

\subsubsection{Conclusions}

Using the GCMC simulations, the absolute and excess isotherms of four gases with different adsorption affinities-argon and methane with high adsorption affinities and neon and helium with low adsorption affinities- are computed. As the pressure increases, the amount of adsorbed gas molecules increases and tend to saturate at high pressures. The absolute adsorption of a particular gas is similar for both 2 and $5 \mathrm{~nm}$ channel heights; For argon and methane, the adsorbed gas amount is higher compared to neon and helium. Excess adsorptions of argon and methane show an optimum pressure below which the excess adsorption increases and above it, the excess adsorption decreases.

DCV-GCMD simulations of chemical-driven flow of the four gases in 2 and $5 \mathrm{~nm}$ channels are performed. Computed normalized velocities are close to one for helium and neon and channel heights, which shows the the velocity profiles are plug-shaped. All the simulations are run in the linear response regime; average velocity in the channel is linear function of the pressure gradient. For all the gases, as the pressure increases, the density and normalized velocity of the molecules at the wall increase. Furthermore, as pressure increases, velocity of the molecules at the first and second bins (the bins closer to the walls) and the ones at the middle of the channel decreases; This is due to the fact that the viscosity of the fluids increase as the Knudsen number decreases (pressure increases). For neon and helium, at pressures below the saturation pressure (a pressure 
beyond which the adsorbed phase density does not change much with pressure) a second layer of molecules is formed. The contribution of the adsorbed gas to the total mass flux across the channel for argon and methane could be more than 50\%. Investigation of the effect of the channel length on the velocity profiles shows that for channels with height-length ratio less than 10 , the results can be masked by the boundary effects. When this ratio increases, the results become less boundary affected and are closer to each other.

\subsection{NEMD Simulations of Pure Gases in Organic Nanochan- nels}

The objective for this work is to investigate the contribution of the adsorbed phase to the mass flux and comparing transport of gases with different adsorption affinities in organic nano-scale channels. In this work, force-driven Non-Equilibrium molecular dynamics (NEMD) simulations are used to compare the transport of gases with high adsorption affinity (Methane and Argon) with the ones with low adsorption affinity (Helium), for channel heights of 2, 4, 6, and 8 nanometers at two Knudsen numbers of 0.1 and 0.2 . Velocity and mass flux profiles across the channel for Argon, Methane, and Helium are compared. Transport diffusion coefficients and molecular flux of these gas are also calculated. Furthermore, adsorption properties are analysed using Grand Canonical Monte Carlo simulations.

For all the gases studied, plug-shaped velocity profiles are observed irrespective of the channel size and Knudsen number. Mass flux profiles of Argon and Methane across the channels demonstrate a significant contribution of adsorbed molecules to the total mass flux. Furthermore, as Knudsen number increases, the contribution of the adsorbed phase to the total mass flux becomes higher. Molecular flux of Helium is smaller than that of Argon and Methane for all channel sizes. The calculated diffusion coefficients of Methane are higher than those for Argon for all the channel sizes and they decrease as the channel size increases. For Argon and Methane, the diffusion coefficients become smaller as Knudsen number increases. For Helium, the diffusion coefficients are weak functions of the channel size and Knudsen number. Based on the results, contribution of the adsorbed molecules can be more than $50 \%$ of the total mass flux of the channel. For the pressure ranges studies, transport diffusivity of Helium is less sensitive to pressure and Knudsen number compared to Argon and Methane. 
This study shows that the transport through organic nano-scale conduits is essentially diffusive. Therefore, to have a realistic model for predicting the recovery of fluids from unconventional resources, the transport equations in organic nanopores should be replaced by the diffusive transport equations.

Comparing the transport properties of different types of gases in nano-scale conduits using NEMD is challenging because these systems have many degrees of freedom, e.g., channel size, molecule size, gas density, and therefore, finding equivalent systems is difficult. Furthermore, for systems under confinement, there is a positional dependence of density and also the state variables, pressure and temperature [103]. Investigation of gas systems with respect to the Knudsen number might be a viable option. The gas flow regimes are characterized by Knudsen number $(K n)$, which is the ratio of mean free path of molecules to the characteristic length of the system,

$$
K n=\frac{1}{\sqrt{2} \pi n H d^{2}},
$$

where $n$ is the number density, $H$ is the channel height, and $d$ is the molecular diameter. This formulation of Knudsen number incorporates molecular size, number density, and channel size and provides a means for comparing systems with different sizes and gas types.

\subsubsection{Molecular Dynamics Methods}

The Grand Canonical Monte Carlo (GCMC) simulations are performed at different pressures for two graphite nanochannel heights of 2 and $4 \mathrm{~nm}$ to determine the gas adsorption isotherms of Methane, Argon, and Helium. In GCMC simulations, the pressure inside the control volume is kept constant by insertion and deletion of molecules. The probability of inserting a molecule is determined as,

$$
p^{+}=\min \left\{\frac{Z V_{C V}}{N_{C V}+1} \exp \left(-\frac{\Delta U}{k_{B} T}\right), 1\right\},
$$

where $Z=\exp \left(\frac{\mu}{k_{B} T}\right) / \Lambda$ is the absolute activity at temperature $T, \Lambda$ is the de Broglie wavelength, $\mu$ is the chemical potential, and $k_{B}$ is the Boltzmann constant. Changes in potential energy resulting from insertion and deletion of molecules are represented by $\Delta U$, volume of control volume is $V_{C V}$, and number of molecules in control volume is $N_{C V}$. Inserted molecules are assigned a velocity using 
Maxwell-Boltzmann distribution. The probability of deleting a molecule is,

$$
p^{-}=\min \left\{\frac{N_{C V}}{Z V_{C V}} \exp \left(-\frac{\Delta U}{k_{B} T}\right), 1\right\}
$$

The temperature of the inserted molecules is determined based on the specified reservoir temperature $(300 \mathrm{~K})$.

Non-equilibrium molecular dynamics (NEMD) simulations of force-driven flow of Argon, Methane, and Helium are performed in three-dimensional graphite channels (with hexagonal lattice) of heights 2, 4, 6, 8 nanometers $(\mathrm{nm})$. The width of the channel is chosen to be $4.5 \mathrm{~nm}$ for all the simulations; however, the channel lengths change with respect to the channel height such that the ratio of the length to height of the channels to be 20 in order to have a fully developed flow. For example, the channel lengths for simulations with $2 \mathrm{~nm}$ and $4 \mathrm{~nm}$ channel heights is selected to be $40 \mathrm{~nm}$ and $80 \mathrm{~nm}$, respectively. The three-layered channel walls are placed in x-y plane. The simulations are performed for two Knudsen numbers of 0.1 and 0.2. The number of molecules for each specific gas and geometry is different and is determined by Eq. 3.8. The OPLS all-atom force field is used to simulate the flow of Methane molecules in nanochannel [49]. The charges of the Hydrogen and Carbon atoms of Methane are set to $0.06 e$ and $0.24 e$, respectively, and the Lennard-Jones parameters are $\sigma_{H}=2.5^{\circ} \mathrm{A}, \epsilon_{H}=0.03 \mathrm{Kcal} / \mathrm{mol}, \sigma_{C}=3.5^{\circ} \mathrm{A}, \epsilon_{C}=0.066 \mathrm{Kcal} / \mathrm{mol}$. A cutoff distance of $9^{\circ} \mathrm{A}$ is considered. The wall temperature is set to $300 \mathrm{~K}$ and the location of its carbon molecules is kept fixed using a spring force. An initial Gaussian velocity distribution with a temperature of $300 \mathrm{~K}$ is considered for the gas molecules and the system's energy is minimized to reach the equilibrium in NVT ensemble (constant number of molecules, constant volume, and constant temperature). A time step of $5 f_{s}\left(10^{-15}\right.$ seconds) and $2.5 f s$ is considered to perform the time integration of equation of motion with Verlet algorithm for Argon (and Helium) and Methane, respectively. In order to induce a constant pressure gradient across channel, driving forces of 0.07 to $0.35 p N\left(10^{-12}\right.$ Newtons $)$ is considered and applied only to the molecules located in the first 3.5 A of the nanochannel (inlet region in Fig. 3.12). The exerted lateral force, $F$, to the molecules is proportional to pressure drop as described by $[15,114]$,

$$
\Delta P \propto \frac{N F}{A},
$$

where $A$ is the area perpendicular to the applied force. Determing the actual pressure gradient in NEMD simulations is not straightforward. Therefore, to compare the molecular fluxes and velocities 
in different pressure gradients, the term $N F / A L$ which is proportional to pressure gradient is used. The number of molecules at the inlet region, $N$, is calculated at each time step and averaged over the total simulation time.

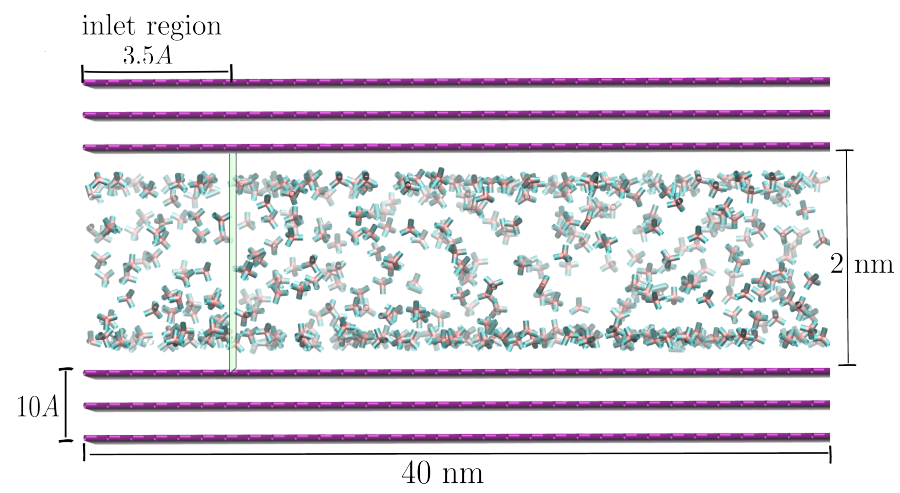

Figure 3.12: Molecular dynamics simulation geometry. The figure is not in scale.

The fluid temperature is fixed at $300 \mathrm{~K}$ using Berendsen thermostat. The damping coefficient of Berendsen thermostat is set to $2 \mathrm{ps}^{-1}$. The simulations are performed for 3 millions time steps which equals to a total simulation time of 15 nanoseconds $(n s)$. All the simulations are performed using Large-scale Atomic/Molecular Massively Parallel Simulator (LAMMPS) [81] and Visual Molecular Dynamics (VMD) [43] is used for the visualizations.

\subsubsection{Results and Discussion}

\section{Equilibrium}

Adsorption isotherms Due to the existence of the attraction forces between the fluid and wall molecules, there is a positional dependence of the fluid density that causes a heterogeneous fluid distribution within the channel. In order to differentiate between the bulk and adsorbed molecules, two quantities are generally defined: Absolute and excess adsorption . Absolute adsorption is the quantity of the gas in the porous structure and excess adsorption is the subtraction of total gas quantity in pore spaces and the gas quantity in the same volume without presence of walls (bulk state).

Absolute and excess adsorption isotherms for the gases in 2 and $4 \mathrm{~nm}$ channels at $300 \mathrm{~K}$ are shown in

Fig. 3.13. The absolute adsorption of all three gases increases as the pressure increases and tend to saturate at high pressures. Methane and Argon show similar absolute loadings, while Helium have 
lower absolute adsorbed amount. The absolute loadings at the different channel sizes are similar for the pressures simulated.

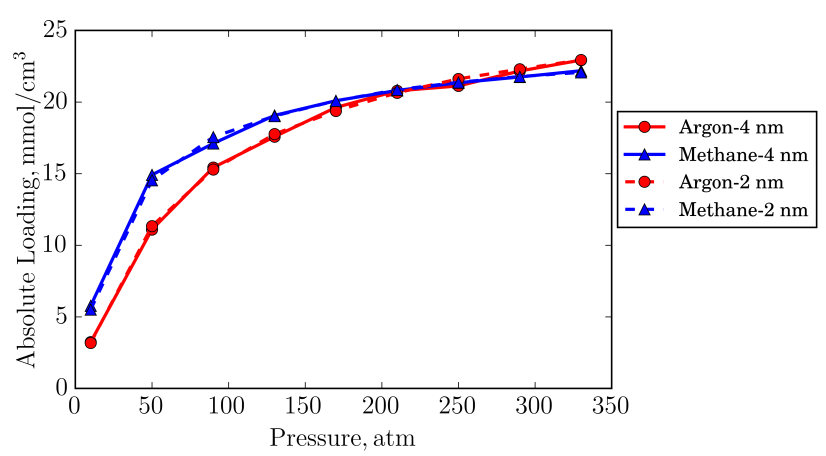

(a) Absolute adsorption

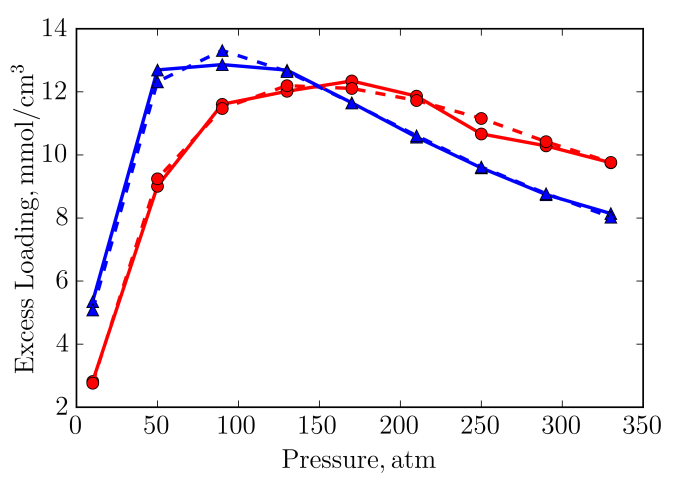

(b) Absolute adsorption

Figure 3.13: Adsorption isotherms of Argon, Methane, and Helium at 2 and $4 \mathrm{~nm}$ height channel at $300 K$. (a) Absolute adsorption (b) Excess adsorption

As pressure increases, the excess loadings increase to reach a maximum value at an optimum pressure and then decreases. This optimum pressure is smaller for Methane than Argon. The maximum excess adsorption for Argon and Methane are 12.2 and $13.3 \mathrm{mmol} / \mathrm{cm}^{3}$, respectively (Fig.3.13). Helium excess adsorption is almost zero and becomes negative for higher pressures ( $P>210 \mathrm{~atm})$. This means that the density of Helium at the wall is equal or less than its density in the bulk phase, which is similar to the results presented by Keller and Staudt [61].

\section{Force-Driven Flow}

Force-driven MD simulations are performed for two Knudsen numbers of 0.1 and 0.2 for Argon, Methane, and Helium for four different graphite channel sizes of 2, 4, 6, and $8 \mathrm{~nm}$. Different forces are applied to the molecules at inlet region of the channel (Table. 3.3). In the following subsections, the velocity profiles, molecular fluxes, and diffusion coefficients of Argon, Methane, and Helium are investigated.

Pressure calculation Determining the exact pressure values in nano-scale channels is challenging in NEMD simulations. However, in GCMC simulations, the pressure can be kept at specific values by the insertion and deletion of the fluid molecules. In order to find the pressure values in the NEMD simulations, a relationship is established between the average gas pressure and the average gas 
Table 3.3: Applied forces to the inlet region of the channels for different gases at Knudsen numbers of 0.1 and 0.2 .

\begin{tabular}{|c|c|c|}
\multicolumn{1}{c|}{} & \multicolumn{2}{c}{ Force $(p N)$} \\
\cline { 2 - 3 } \multicolumn{1}{c|}{} & $\mathrm{Kn}=0.1$ & $\mathrm{Kn}=0.2$ \\
\hline \multirow{2}{*}{ Argon } & 0.139 & 0.208 \\
\cline { 2 - 3 } Methane & 0.208 & 0.278 \\
\cline { 2 - 3 } & 0.278 & 0.347 \\
\hline \multirow{3}{*}{ Helium } & 0.07 & 0.139 \\
\cline { 2 - 3 } & 0.139 & 0.208 \\
\cline { 2 - 3 } & 0.208 & 0.278 \\
\hline
\end{tabular}

Table 3.4: Pressures for each simulation setup.

\begin{tabular}{|c|c|c|c|c|c|c|}
\hline \multirow{2}{*}{$\begin{array}{c}\text { Size } \\
(\mathrm{nm})\end{array}$} & \multicolumn{6}{|c|}{ Pressure (atm) } \\
\cline { 2 - 7 } & \multicolumn{3}{|c|}{ Kn=0.1 } & \multicolumn{3}{c|}{ Kn=0.2 } \\
\cline { 2 - 7 } & Argon & Methane & Helium & Argon & Methane & Helium \\
\hline 2 & 430 & 240 & 1000 & 195 & 120 & 380 \\
\hline 4 & 190 & 135 & 380 & 97 & 72 & 175 \\
\hline 6 & 127 & 93 & 240 & 58 & 44 & 113 \\
\hline 8 & 99 & 72 & 175 & 50 & 40 & 85 \\
\hline
\end{tabular}

density for each gas at each channel size based on the GCMC simulation results. As an example, the relationship between the average pressure and density of Argon for a 2-nm channel is demonstrated in Fig. 3.14. Pressure values at different channel heights for Argon, Methane, and Helium are listed in Table. 3.4.

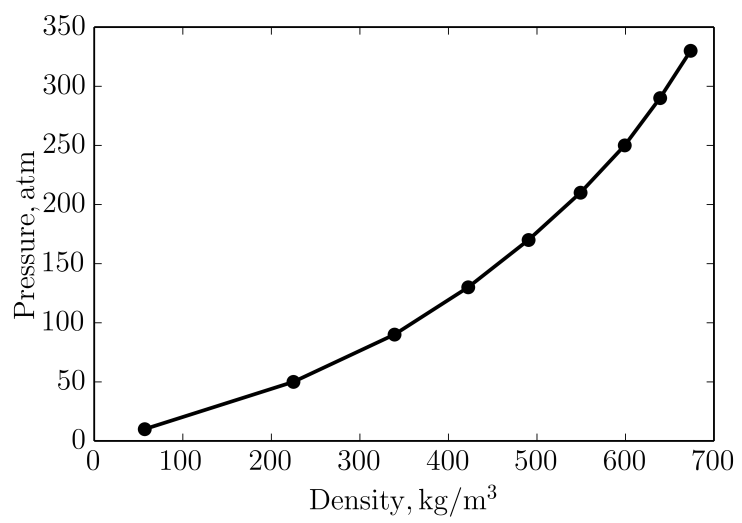

Figure 3.14: Relationship between the average density and pressure of Argon for the 2- $n m$ channel.

Velocity and Density Profiles The simulation results of velocity and density profiles of Methane, Argon, and Helium are presented in Figs. 3.15, 3.16, and 3.17, respectively. For each channel height and gas type, the results are plotted for two Knudsen numbers of 0.1 and 0.2 . The velocity profiles 
are shown with solid blue lines for $K n=0.2$ and solid red lines for $K n=0.1$. The density profiles are shown with blue and red dashed lines for Knudsen numbers 0.2 and 0.1, respectively.

The bulk and adsorbed densities of each gas decreases as the channel height increases for each Knudsen number. This is due to the fact that as channel height increases, the number density decreases to maintain the same Knudsen number for all channel sizes (Eq. 3.8). The densities of Argon and Methane at the wall (and similarly average density across the channel height) do not decrease linearly with increasing the channel height. For instance at $K n=0.1$, the average density of Methane across the channel height decreases from $185 \mathrm{~kg} / \mathrm{m}^{3}$ in 2-nm channel to $53 \mathrm{~kg} / \mathrm{m}^{3}$ in 8-nm channel. Similarly, average density of Argon decreases by 3.9 times (from 642 to $164 \mathrm{~kg} / \mathrm{m}^{3}$ ) from the 2-nm to 8-nm channel.

To compare the velocities of a gas at different Knudsen numbers, same pressure drop is applied. In NEMD simulations, pressure gradients are proportional to number of molecules and the force applied over the channel length (Eq. 3.13). For any channel size, the number of molecules for a simulation with $K n=0.1$ is twice those in simulations with $K n=0.2$ for each gas. Therefore, the forces applied to the molecules in the simulations with $K n=0.2$ should be twice of the simulation with $K n=0.1$ to have the same pressure gradients applied. In the velocity profiles plotted in Figs. 3.15 and 3.16, the applied forces are 0.139 and $0.278 p N$ for $K n=0.1$ and $K n=0.2$, respectively. The applied forces on Helium molecules (Fig. 3.17) are 0.07 and $0.139 p N$ for $K n=0.1$ and $K n=0.2$, respectively. 

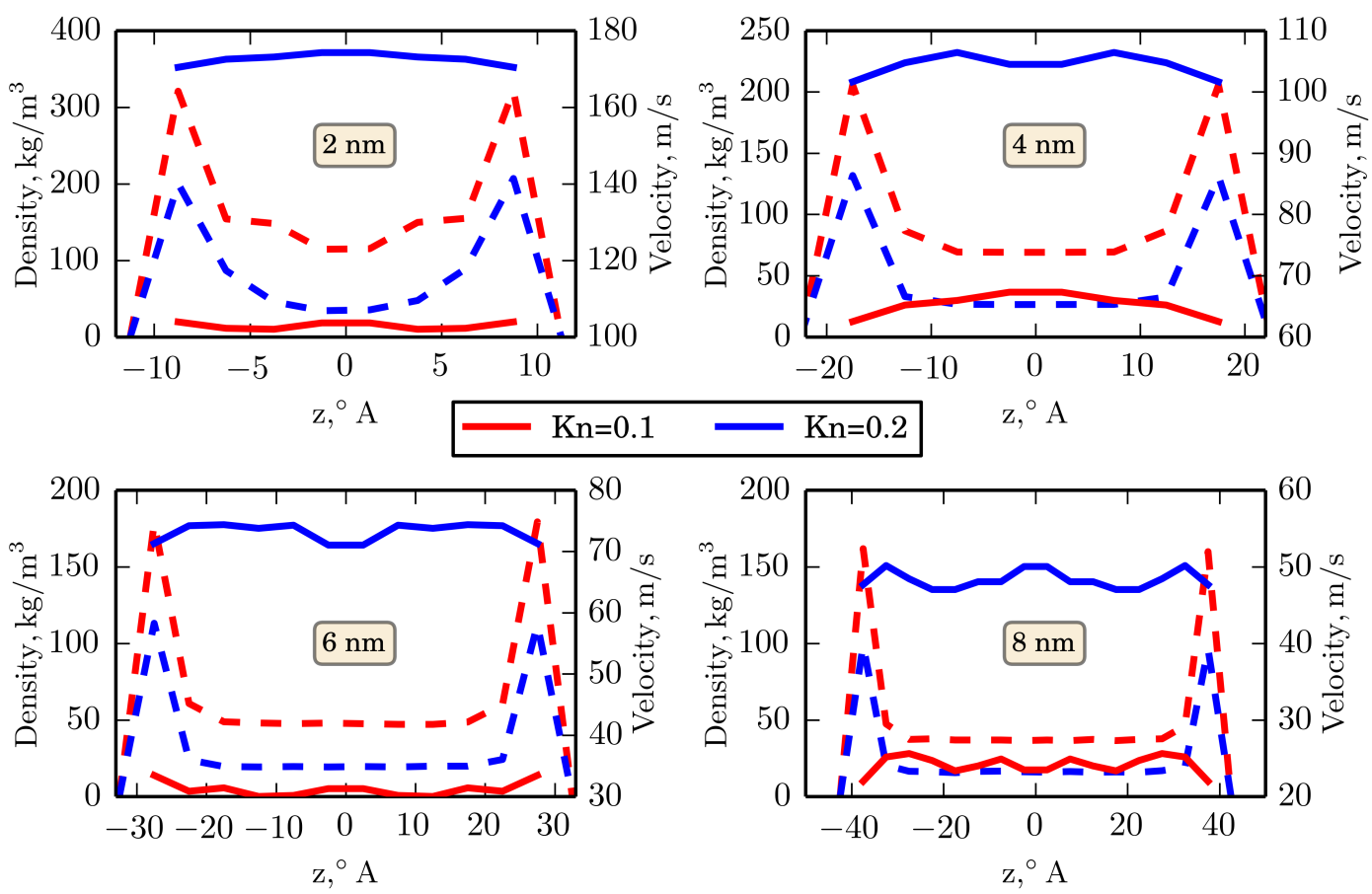

Figure 3.15: Velocity and density profiles of Methane in the graphite channels.
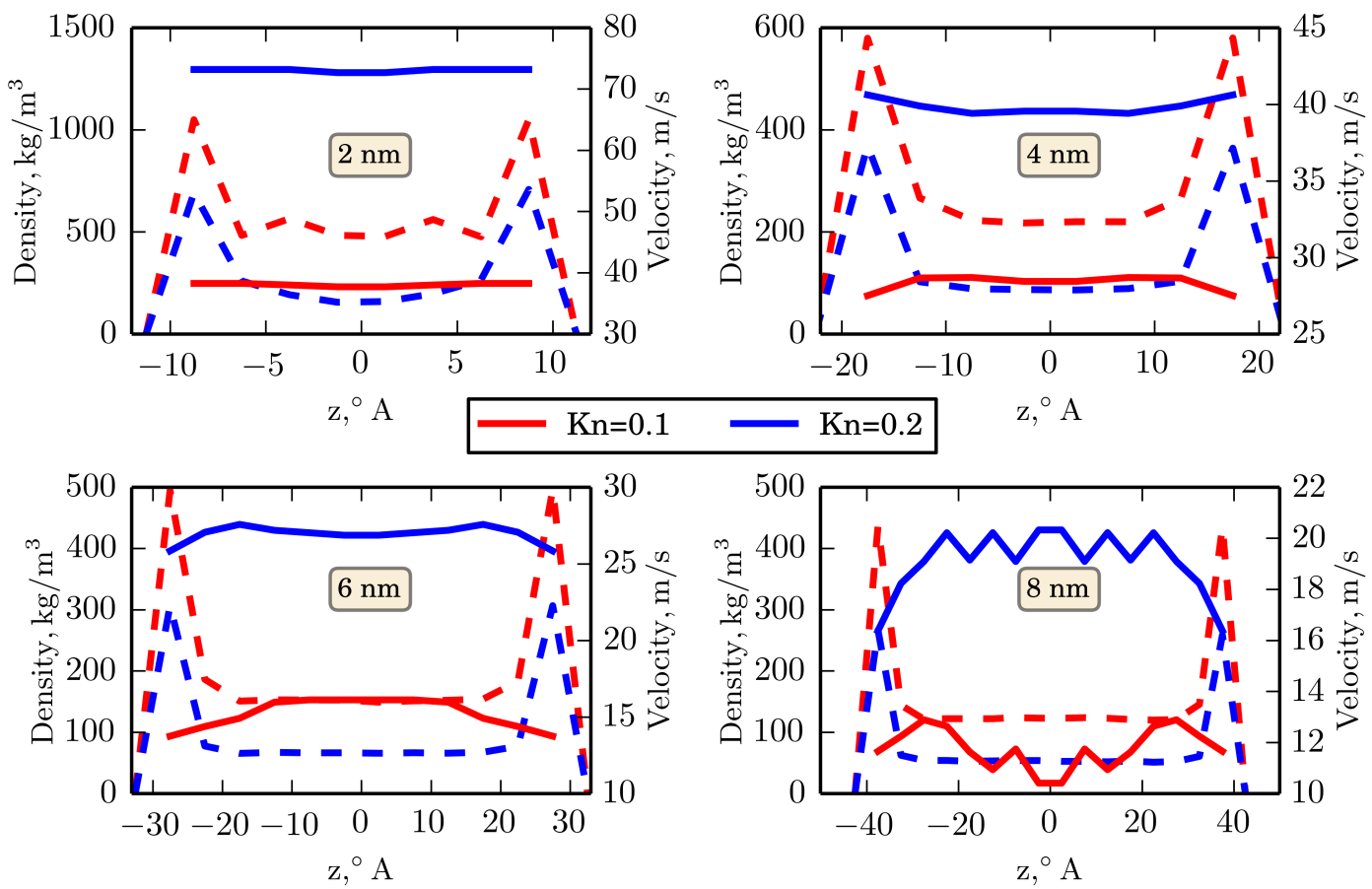

Figure 3.16: Velocity and density profiles of Argon in the graphite channels. 

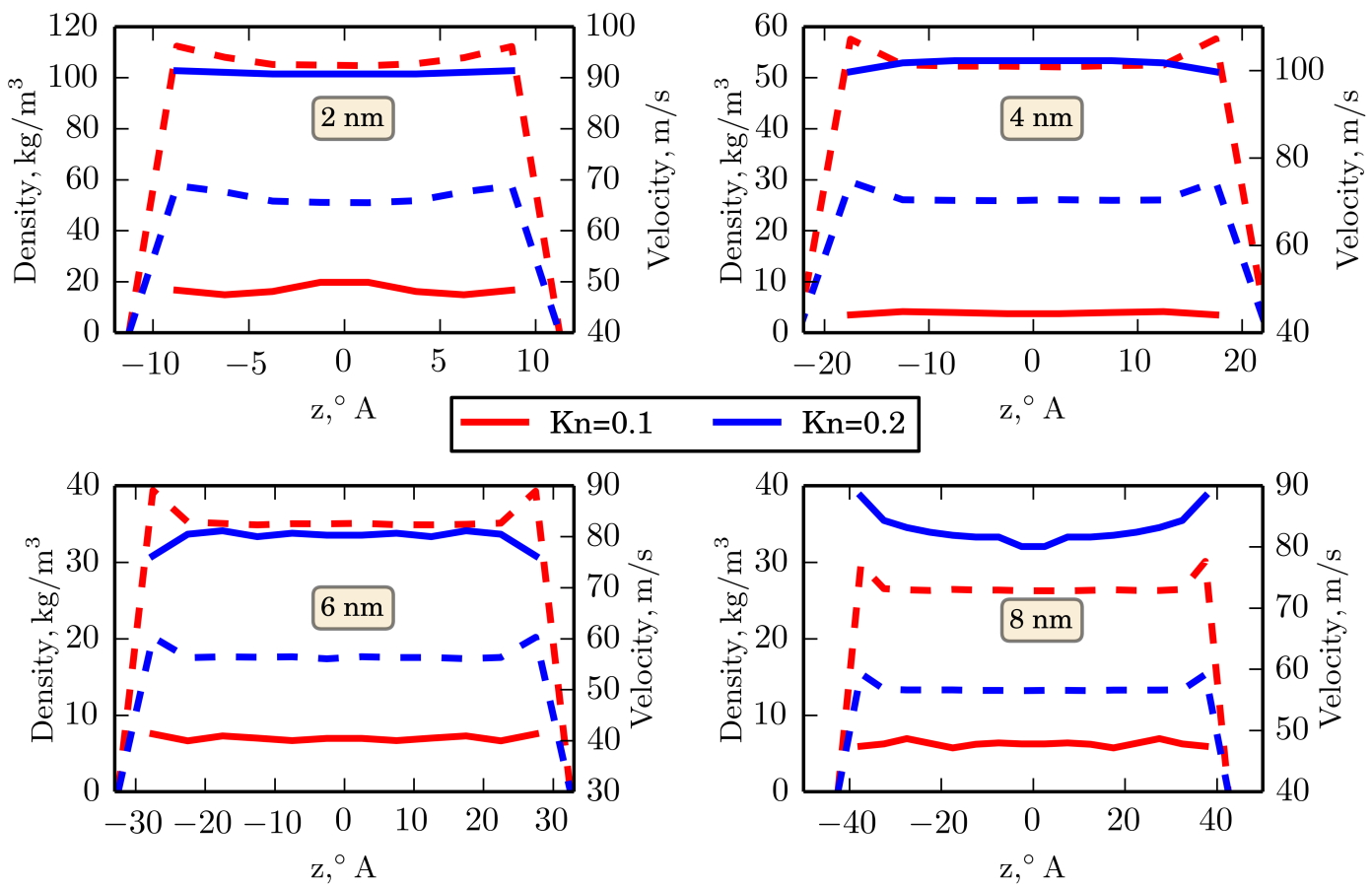

Figure 3.17: Velocity and density profiles of Helium in the graphite channels.

For all gases and channel sizes, whether the adsorption affinity is high (Argon and Methane) or low (Helium), plug-shaped velocity profiles are established. In other words, the adsorbed Argon and Methane molecules flow with similar velocities as the gas molecules in middle of channel despite their higher density at the wall. Therefore, the adsorbed phase contribute significantly to the flow. Additionally, for a particular channel height, the higher the Knudsen number, the higher the average gas velocity at the same pressure drop. This result is consistent with predictions of mathematical models of flow in nano-scale channels, e.g., R13 moment method [55].

Molecular Fluxes The molecular fluxes of Methane, Argon, and Helium are plotted in Figs. 3.18a, $3.18 \mathrm{~b}$, and $3.18 \mathrm{c}$ against the pressure gradients for all the channel sizes. For comparison purposes, the pressure drop parameter, $\frac{N F}{A L}$, which is linearly proportional to the pressure gradient, is used. The molecular fluxes are calculated by multiplying the average channel densities by the their average velocities.

For all the gases, the molecular flux increases with increasing the pressure gradient for all the channel sizes. Furthermore, for a specific pressure gradient, the molecular flux of the gases decreases as channel the sizes increase. For all the pressure gradients studied, the highest molecular flux is 
obtained for the 2-nm channel. For channel sizes less than $8 \mathrm{~nm}$, molecular flux values and the trend of their increase with increasing the pressure gradient is approximately the same for Argon and Methane. For the 8-nm channel, although differences are small, molecular fluxes for Methane are higher than those for Argon.

Molecular fluxes for Helium are smaller than those for Argon and Methane for all channel sizes. The molecular fluxes of Argon and Methane are similar to each other but different from Helium. Molecular fluxes increase from 5050 to $38577 \mathrm{~kg} / \mathrm{m}^{2} \mathrm{~s}$ for Argon and from 4498 to $37244 \mathrm{~kg} / \mathrm{m}^{2} \mathrm{~s}$ for Methane as pressure gradient parameter increases from 0.26 to $2.56 \mathrm{psi} / \mathrm{nm}$ for Fig. 3.18a. For the same channel heights, the Helium molecular flux increases from 2725 to $9000 \mathrm{~kg} / \mathrm{m}^{2} \mathrm{~s}$ as the pressure gradient parameter increases from 0.35 to $2.78 \mathrm{psi} / \mathrm{nm}$.

According to the results presented in Figs. 3.18, the molecular flux is approximately a linear function of the pressure gradient for each gas in the channels. Furthermore, for Argon and Methane which have similar adsorption behaviors, the rate of change of molecular flux with respect to the pressure gradient is approximately the same. This shows that the mass transfer through organic nanoscale conduits is essentially diffusive. This has significant implications in reservoir modeling of unconventional resources because the governing equation used in fluid flow through porous media, Darcy's equation, is derived based on the advective flow assumptions. To have a realistic model for predicting the recovery of fluids from unconventional resources, the transport equations in organic nanopores should be replaced by the diffusive transport equations. 


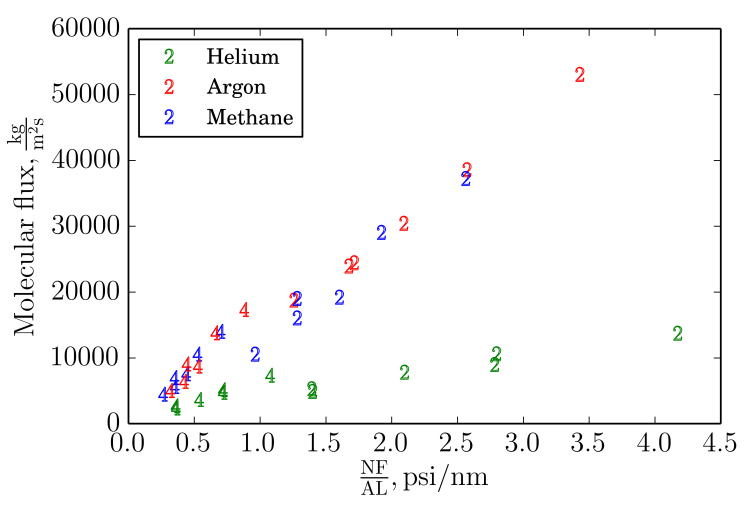

(a) 2 and $4 \mathrm{~nm}$

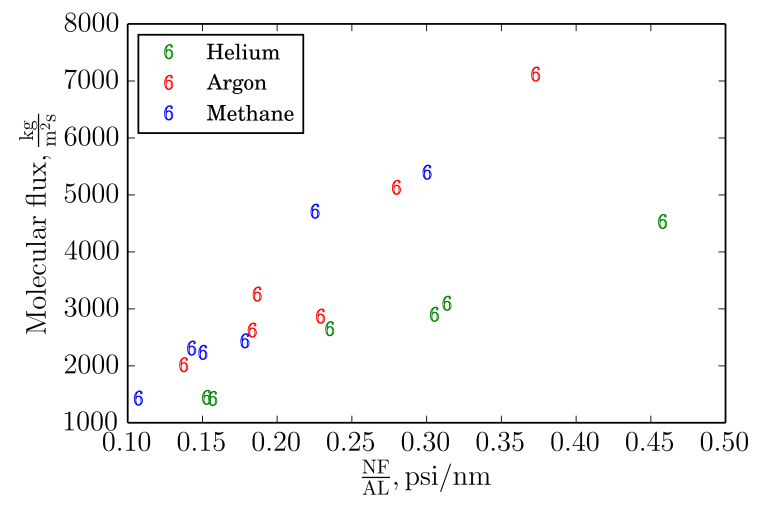

(b) $6 \mathrm{~nm}$

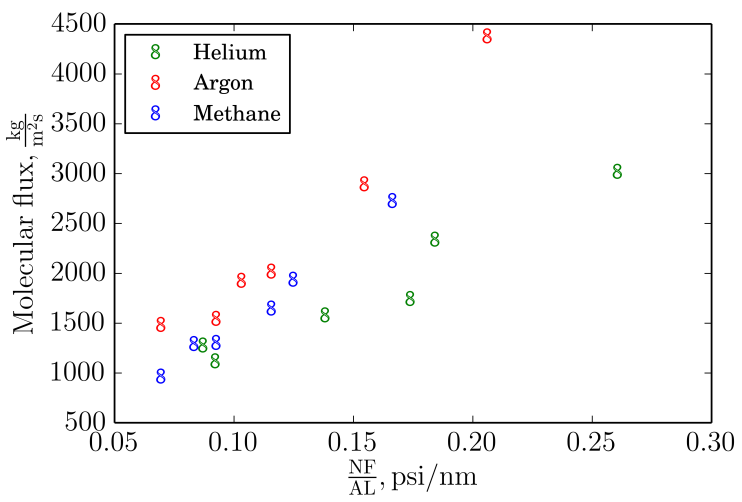

(c) $8 \mathrm{~nm}$

Figure 3.18: Molecular flux vs. pressure gradient for (a) 2 and $4 \mathrm{~nm}$, (b) $6 \mathrm{~nm}$, and (c) $8 \mathrm{~nm}$.

Mass Flux Profiles In order to compare the mass flux profiles of the gases, the channel is divided to equally-sized bins. At each bin, the average velocity is multiplied by the average density and divided by the product of average velocity and density across the channel height to determine the dimensionless mass flux. The calculated dimensionless mass flux profiles of Argon, Methane, and Helium are plotted for the Knudsen numbers of 0.1 and 0.2 in Fig. 3.19. Mass flux at the first and last bin, i.e., bins next to the channel walls, have higher dimensionless mass flux values for Argon and Methane compared the ones for the bins away from the walls. This is due to the fact that the density of these two gases are higher near the wall than those of the bulk phase. Mass flux profile of Helium are plug-shaped as the density and velocity values remain approximately constant across the channel height

According to these results, as Knudsen number increases, the contribution of the adsorbed phase to 
the total mass flux becomes higher. These contributions are $42 \%$ for both Argon and Methane at $\mathrm{Kn}=0.1$, which increases to approximately $53 \%$ at $\mathrm{Kn}=0.2$.

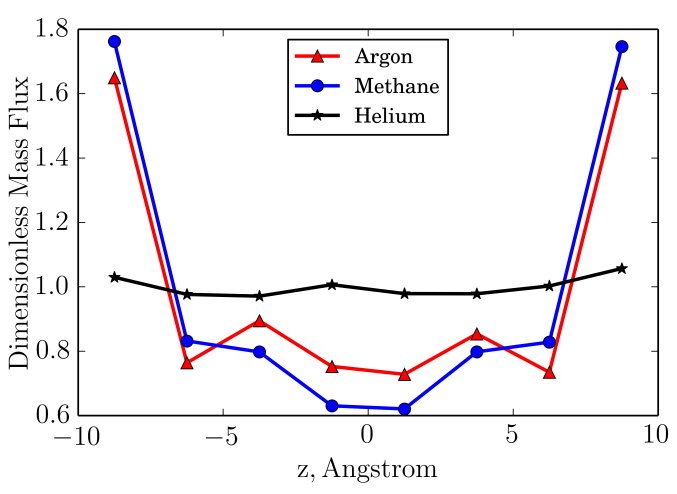

(a) $\mathrm{Kn}=0.1$

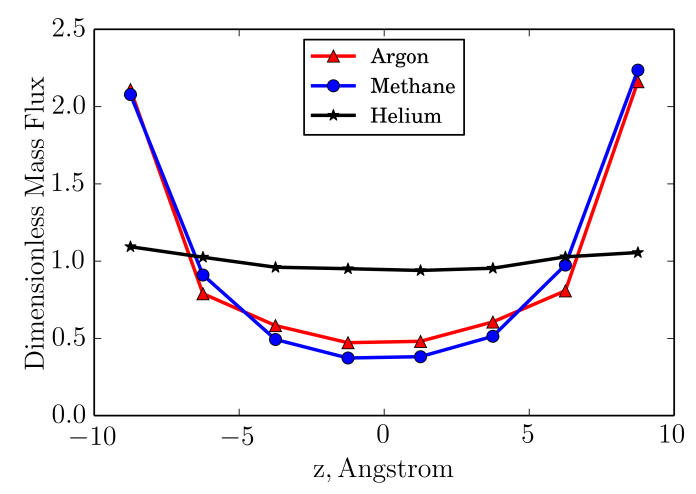

(b) $\mathrm{Kn}=0.2$

Figure 3.19: Dimensionless mass flux profiles of Argon, Methane, and Helium at (a) Kn=0.1 (b) $\mathrm{Kn}=0.2$.

Diffusion For NEMD simulations, the transport coefficients can be calculated from the measured molecular flux as [11],

$$
D_{t}=\frac{k_{B} T j}{\hat{\rho} F}
$$

where $k_{B}$ is the Boltzmann constant, $T$ is the temperature, $j$ is the molecular flux, $\hat{\rho}$ is the average gas density in the channel, and $F$ is the applied external force.

The transport diffusivity coefficients are plotted for Knudsen numbers of 0.1 and 0.2 for Argon, Methane, and Helium in Fig. 3.20. As mentioned earlier, three different external forces are applied for each channel height at each Knudsen number. The error bars are plotted in Fig. 3.20 show the variance of diffusion coefficients at different external forces. The transport diffusivity coefficients listed in Table. 3.5 are the average diffusivity coefficients calculated for the external forces.

For each gas, the highest transport coefficient corresponds to the smallest channel, i.e., $2 \mathrm{~nm}$. For Methane and Argon, transport coefficients decrease as the channel size increases. The diffusion coefficients of Methane are higher than those for Argon for all the channel sizes. For Argon and Methane, the diffusion coefficients are higher at $K n=0.1$ compared with $K n=0.2$ for the same channel size. For Methane, the diffusion coefficients decrease 76 and $70 \%$ as the channel sizes increase from 2 to $8 \mathrm{~nm}$ for Knudsen numbers of 0.1 and 0.2, respectively. For Argon, this decrease is 69 and $72 \%$ for Knudsen numbers of 0.1 and 0.2 , respectively. For Helium, the diffusion coefficients 
are weak functions of the channel size and Knudsen number. The change in diffusion coefficients for Helium is less that $15 \%$. The average diffusivity coefficients for Helium at $K n=0.2$ are slightly higher than $K n=0.1$ for the same channel size.

Table 3.5: Transport diffusion coefficients for Methane, Argon, and Helium at Kn=0.1 and Kn0.2 for all the channel sizes.

\begin{tabular}{|c|c|c|c|}
\hline Gas & Knudsen number & $\begin{array}{c}\text { Channel Height } \\
(\mathrm{nm})\end{array}$ & $\begin{array}{l}\text { Diffusion Coefficient } \\
\qquad\left(10^{-6} \mathrm{~m}^{2} / \mathrm{s}\right)\end{array}$ \\
\hline \multirow{8}{*}{ Methane } & \multirow{4}{*}{0.1} & 2 & 3.06 \\
\hline & & 4 & 1.95 \\
\hline & & 6 & 1.12 \\
\hline & & 8 & 0.732 \\
\hline & \multirow{4}{*}{0.2} & 2 & 2.43 \\
\hline & & 4 & 1.62 \\
\hline & & 6 & 0.969 \\
\hline & & 8 & 0.720 \\
\hline \multirow{8}{*}{ Argon } & \multirow{4}{*}{0.1} & 2 & 1.18 \\
\hline & & 4 & 0.834 \\
\hline & & 6 & 0.474 \\
\hline & & 8 & 0.366 \\
\hline & \multirow{4}{*}{0.2} & 2 & 1.11 \\
\hline & & 4 & 0.622 \\
\hline & & 6 & 0.387 \\
\hline & & 8 & 0.315 \\
\hline \multirow{8}{*}{ Helium } & \multirow{4}{*}{0.1} & 2 & 2.66 \\
\hline & & 4 & 2.75 \\
\hline & & 6 & 2.45 \\
\hline & & 8 & 2.35 \\
\hline & \multirow{4}{*}{0.2} & 2 & 2.85 \\
\hline & & 4 & 2.79 \\
\hline & & 6 & 2.64 \\
\hline & & 8 & 2.46 \\
\hline
\end{tabular}

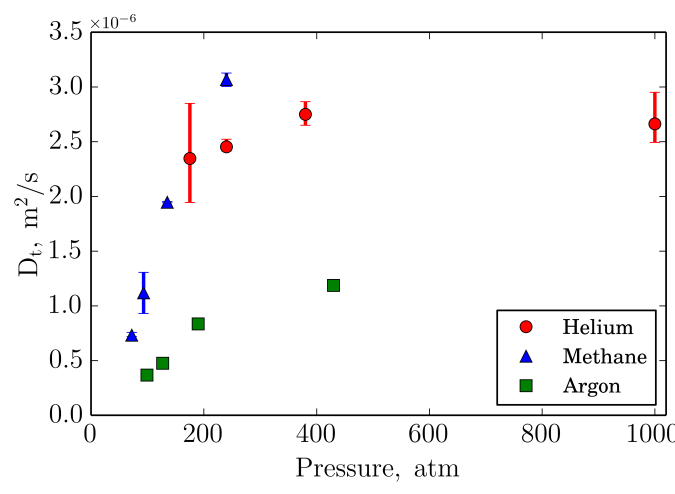

(a) $\mathrm{Kn}=0.1$

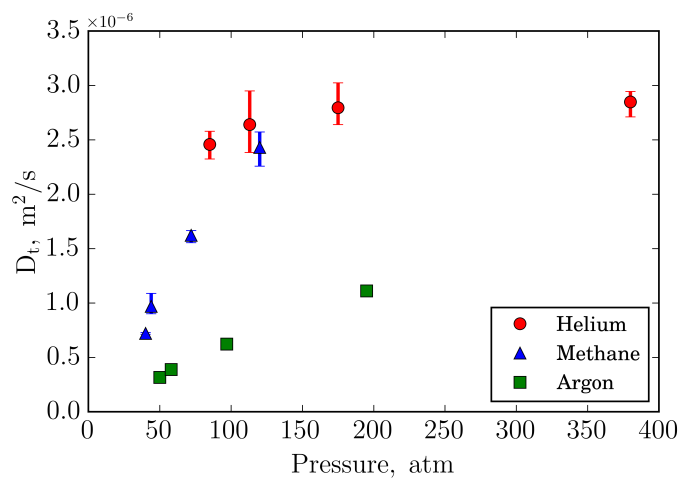

(b) $\mathrm{Kn}=0.2$

Figure 3.20: Transport diffusion coefficients at (a) $\mathrm{Kn}=0.1$ and (b) $\mathrm{Kn}=0.2$. 


\subsubsection{Conclusions}

Adsorption and force-driven transport of Helium, Argon, and Methane in four graphite channels of 2, 4, 6, and $8 \mathrm{~nm}$ hight is studied by performing GCMC and NEMD simulations. Methane and Argon show similar adsorption behavior (absolute and excess adsorptions) while the density of Helium across the channel height is approximately constant.

For all gases, whether the adsorption affinity is high (Argon and Methane) or low (Helium), plugshaped velocity profiles are established for all the channel heights. Therefore, due to the higher adsorbed density values of the Argon and Methane, the adsorbed phase contribute significantly to the overall mass flux across the channel. Mass flux profiles of Argon and Methane across the channels demonstrate a significant contribution of adsorbed molecules to total mass flux. According to these results, as Knudsen number increases, the contribution of the adsorbed phase transport to the total mass flux of the channels becomes higher. This contribution is $42 \%$ for Argon and Methane at Knudsen number of 0.1 , which increases to approximately $53 \%$ at Knudsen number of 0.2 .

For the gases studied, the highest molecular flux is obtained for the 2-nm channel. Molecular flux of Helium is smaller than that of Argon and Methane for all the channel sizes. For channel sizes less than $8 \mathrm{~nm}$, molecular flux values and the trend of their increase with increasing the pressure gradient is approximately the same for Argon and Methane. For the 8-nm channel, molecular flux for Methane is slightly higher than that for Argon.

For each gas, the highest transport coefficient corresponds to the smallest channel, i.e., $2 \mathrm{~nm}$. The diffusion coefficients of Methane are higher than those for Argon for all the channel sizes and they decrease as the channel size increases. For Argon and Methane, the diffusion coefficients decrease as the Knudsen number increases. For Helium, the diffusion coefficients are weak functions of the channel size and Knudsen number.

This study shows that the transport through organic nano-scale conduits is essentially diffusive. This has significant implications in reservoir modeling of unconventional resources because the governing equation used in fluid flow through porous media, Darcy's equation, is derived based on the advective flow assumptions. Therefore, to have a realistic model for predicting the recovery of fluids from

unconventional resources, the transport equations in organic nanopores should be replaced by the diffusive transport equations. 


\subsection{NEMD Simulations of Multicomponent Gases in Rough Organic Nanochannels}

Most of the current studies on the transport of fluids in shale are focused on the transport of singlecomponent fluids, mainly methane. However, there are a wide range of fluids present in natural gas systems in shale, e.g., methane, ethane, propane, butane, nitrogen, and carbon dioxide. Due to the differences in the sorption properties of different components in these systems, they do not uniformly distributed across the pore. Heavier components tend to accumulate in the vicinity of the pore walls and may trap within the pore and remain in the reservoir, while the lighter components can be produced. The objective for this study is to further enhance the fundamental understanding of transport of natural gas systems in shale by investigating the adsorption and transport of two different gas samples with two different compositions.

There have been some studies on the adsorption and transport of multi-component hydrocarbon mixtures in shale organic matter using MD. Ma and Jamili [68] used Simplified Local-Density theory coupled with Modified Peng-Robinson Equation of State to predict the density profiles of pure and mixture hydrocarbons in confined pores. They found that the compositions of the fluid mixtures are not uniformly distributed across the pore. Heavier component (n-butane) tended to accumulate near the wall while lighter component (methane) would tend to stay in the center region of the pore. [25] used EMD and boundary driven non-equilibrium molecular dynamics (BD-NEMD) simulations to study the transport of fluid mixtures for different chemical compositions through a molecular model

of kerogen type II. They computed Onsagers coefficients for pure and multi-component mixture of fluids. The results suggested that the flow inside kerogen is of diffusive nature and that the transport properties can be calculated using EMD.

In this work, non-equilibrium molecular dynamics (NEMD) is used to simulate the adsorption behavior and chemical potential-driven flow of multi-component fluids in nano-scale carbon channels. The density profiles of the each component within the channel is calculated across the channel width and the selectivity of heaviest molecule (hexane) over other gas components are determined. Furthermore, velocity, mass flux, and diffusion coefficients of each component in the channels are calculated. 


\subsubsection{Computational Methodology}

Non-equilibrium molecular dynamics (NEMD) simulations of force-driven flow of two gas mixtures (Table 3.6) are performed in three-dimensional graphite channels with heights of 2 and 4 nanometers $(\mathrm{nm})$. The width of the channel is $4.5 \mathrm{~nm}$ for all the simulations, while the channel lengths change such that the ratio of the length to height of the channel remain 20 to achieve a fully developed flow. For instance, the channel lengths for simulations with $2 \mathrm{~nm}$ and $4 \mathrm{~nm}$ heights are $40 \mathrm{~nm}$ and $80 \mathrm{~nm}$, respectively. Moltemplate molecular builder software [46] is used to create the graphite channels. The channels consist of three graphite layers that are placed in $x-y$ plane. The distance between graphite layers is $0.335 \mathrm{~nm}$. The distance between two adjacent carbon atoms in the same graphite layer is 0.142 .

In order to simulate surface roughness, atoms are deleted from the most inner layers (layers in contact with the gas molecules (Fig. 3.21). Total number of molecules for both gas samples is the same $(N=2028)$ but the molar percentage of two samples are different. Sample 2 has a higher density because the molar percentage of the heavier molecules $(M W>16 \mathrm{gr} / \mathrm{mol})$ is higher. Compositions of both samples are listed in Table. 3.6.

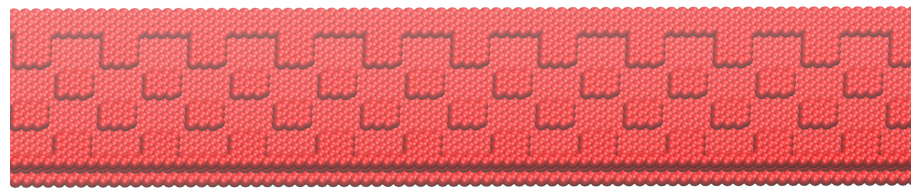

Figure 3.21: Representation of the rough surfaces used in the simulations.

The OPLS all-atom force field is used to simulate the flow of gas molecules in the channels [49]. The charges of hydrogen and carbon atoms of methane are set to $0.06 e$ and $-0.24 e$, respectively. For $\mathrm{CH}_{3}$ groups, the charges are $-0.18 e$ and $0.06 e$ for carbon and hydrogen, respectively. For $\mathrm{CH}_{2}$ groups, these charges are $-0.12 e$ for carbon and $0.06 e$ for hydrogen. Lennard-Jones parameters of carbon and hydrogen in all organic molecules are $\sigma_{H}=2.5^{\circ} \mathrm{A}, \epsilon_{H}=0.03 \mathrm{Kcal} / \mathrm{mol}, \sigma_{C}=3.5^{\circ} \mathrm{A}$, and $\epsilon_{C}=0.066 \mathrm{Kcal} / \mathrm{mol}$. A cut-off distance of $9^{\circ} A$ is considered. The wall temperature is set to $300 \mathrm{~K}$ and the location of its carbon molecules is kept fixed using a spring force. An initial Gaussian velocity distribution with a temperature of $300 \mathrm{~K}$ is considered for the gas molecules and the system's energy is minimized to reach the equilibrium in NVT ensemble (constant number of molecules, constant volume, and constant temperature). A time step of 1.5 fs (10 $10^{-15}$ seconds) is considered to perform the time integration of equation of motion with Verlet algorithm. 
Table 3.6: Composition of the gas samples used for simulations.

\begin{tabular}{lcc} 
& \multicolumn{2}{c}{ Gas Composition (\%) } \\
\hline Gas & Sample 1 & Sample 2 \\
\hline \hline $\mathrm{CH}_{4}$ & 92 & 83 \\
\hline $\mathrm{C}_{2} H_{6}$ & 5 & 10 \\
\hline $\mathrm{C}_{3} H_{8}$ & 1.2 & 3.7 \\
\hline $\mathrm{C}_{4} H_{10}$ & 0.2 & 0.4 \\
\hline $\mathrm{C}_{5} H_{12}$ & 0.2 & 0.4 \\
\hline $\mathrm{C}_{6} H_{14}$ & 0.2 & 0.4 \\
\hline $\mathrm{CO}_{2}$ & 0.4 & 0.6 \\
\hline$N_{2}$ & 0.6 & 0.9 \\
\hline $\mathrm{O}_{2}$ & 0.2 & 0.6 \\
\hline
\end{tabular}

In order to induce a constant pressure gradient across channel, driving forces of 0.67 to $6.67 \mathrm{pN}$ $\left(10^{-12}\right.$ Newtons $)$ are applied only to the molecules located in the first $3.5 \mathrm{~A}$ of the channel. The exerted lateral force, $F$, to the molecules is proportional to the pressure drop as described by Carr et al. [15], Zhu et al. [114],

$$
\Delta P \propto \frac{N F}{A}
$$

where $A$ is the area perpendicular to the applied force. Determining the actual pressure gradient in NEMD simulations is not straightforward. Therefore, to compare the molecular fluxes and velocities in different pressure gradients, the term $N F / A L$, which is proportional to pressure gradient, is used. The number of molecules at the inlet region, $N$, is calculated at each time step and averaged over the total simulation time.

The fluid temperature is fixed at $300 \mathrm{~K}$ using Nose-Hoover thermostat with a damping coefficient of $2 \mathrm{ps}^{-1}$. The simulations are performed for 5 millions time steps which equals to a total simulation time of 7.5 nanoseconds $(n s)$. All the simulations are performed using Large-scale Atomic/Molecular Massively Parallel Simulator (LAMMPS) [81] and Visual Molecular Dynamics (VMD) [43] is used for the visualizations. 


\subsubsection{Results and Discussion}

\section{Density Profiles}

For both channel sizes, the gas densities near the wall are higher compared to the ones in the middle of channel (Fig. 3.22). Two density peaks can be observed for both channel sizes in both samples. The first peaks (approximately at $z=7$ and $33^{\circ} A$ for $2 \mathrm{~nm}$ channel) represent the gas molecules adsorbed to the two-layered graphite sections of the channel (sections of the channel where the carbon atoms are taken out) (Fig. 3.21). The second peaks (approximately at $z=12.5$ and $28.5^{\circ} \mathrm{A}$ for $2 \mathrm{~nm}$ channel) represent the density of gas molecules adsorbed to the three-layered sections of the surface, where no carbon atoms are removed from the surface. A third peak is started to form approximately at $z=16^{\circ} \mathrm{A}$.

Density of sample 1 is lower near the walls, which is due to the fact that it contains lighter molecules compared with that of sample 2 (Table 3.6). For $2 \mathrm{~nm}$ channel, the average gas densities are 134 and $147 \mathrm{~kg} / \mathrm{m}^{3}$ for samples 1 and 2 , respectively. In $4 \mathrm{~nm}$ channel, the average gas densities are 75 $\mathrm{kg} / \mathrm{m}^{3}$ for sample 1 and $83 \mathrm{~kg} / \mathrm{m}^{3}$ for sample 2 .

Based on the simulations results, for butane, pantane, and hexane, five out of eight molecules (for sample 2) are adsorbed to the walls. Also, half of propane molecules $(N=74)$ are adsorbed to the graphite walls. The density profiles of each gas component in the samples are plotted in Fig. 3.23. Furthermore, the density of heavier components (hexane, pantane, butane, and propane) in middle of channel is very low for both channel sizes and compositions. In order to compare the tendency of different components to be adsorbed to the wall, selectivity of hexane over other gas components is determined,

$$
S_{C_{6} H_{24} / C_{n} H_{2 n+2}}=\frac{x_{C_{6} H_{24}} / x_{C_{n} H_{2 n+2}}}{y_{C_{6} H_{24}} / y_{C_{n} H_{2 n+2}}}, \quad \text { with } \quad 0<n<6
$$




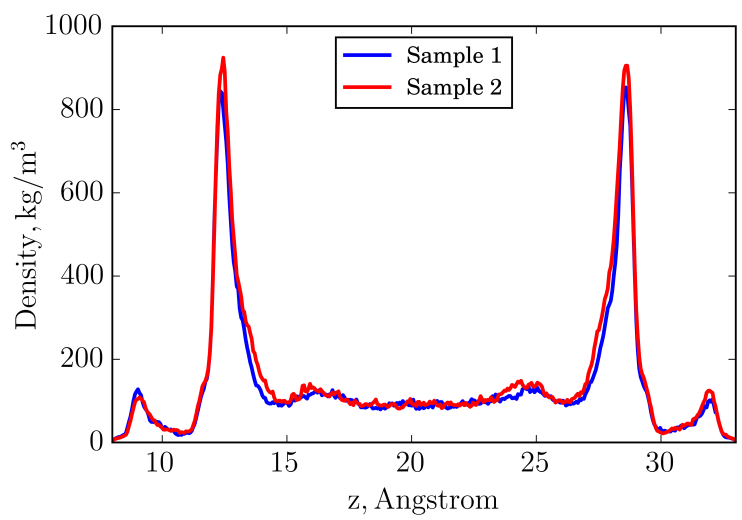

(a) $2 \mathrm{~nm}$

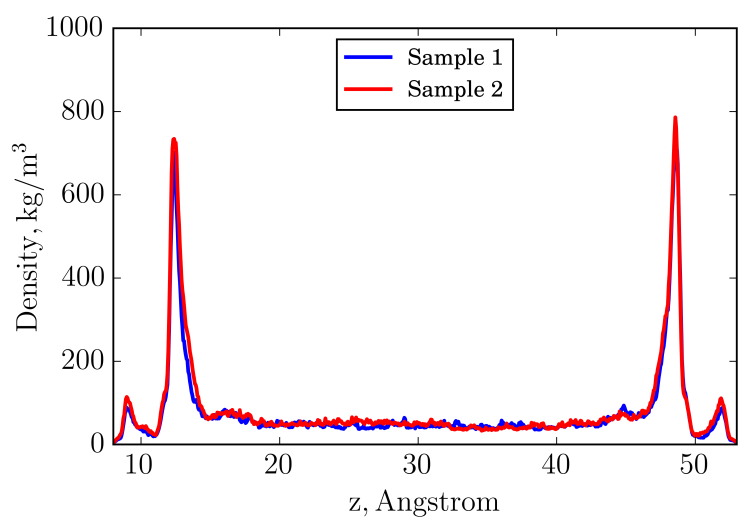

(b) $4 \mathrm{~nm}$

Figure 3.22: Density profiles of the two samples in (a) $2 \mathrm{~nm}$ and (b) $4 \mathrm{~nm}$ channels.

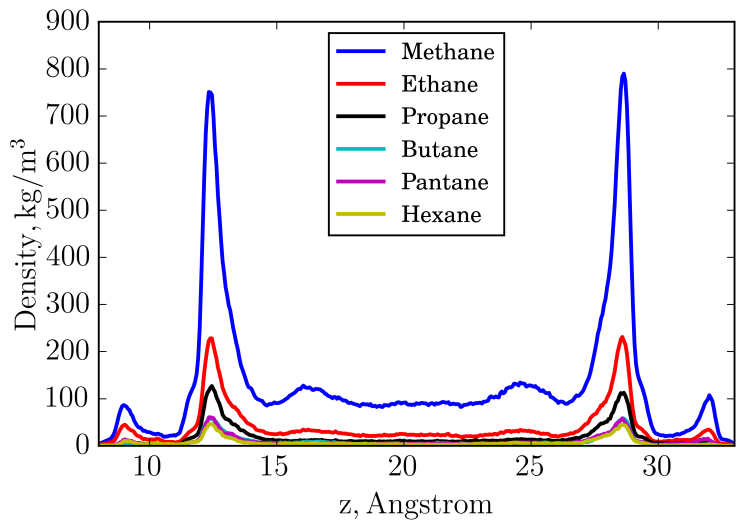

(a) $2 \mathrm{~nm}$

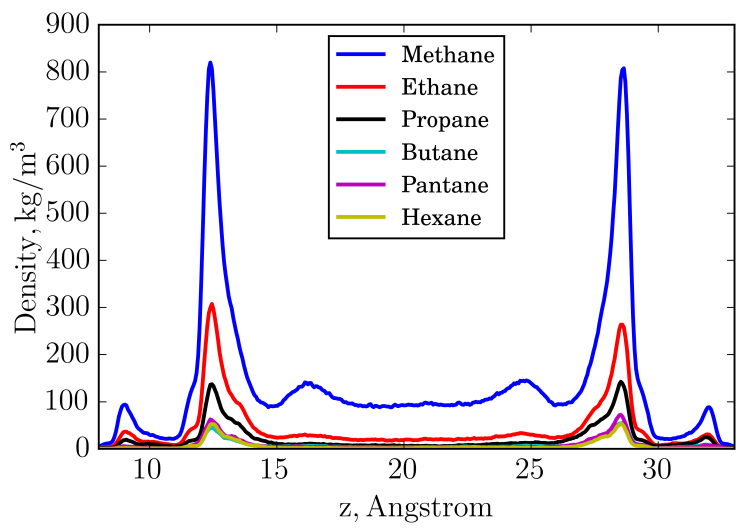

(c) $2 \mathrm{~nm}$

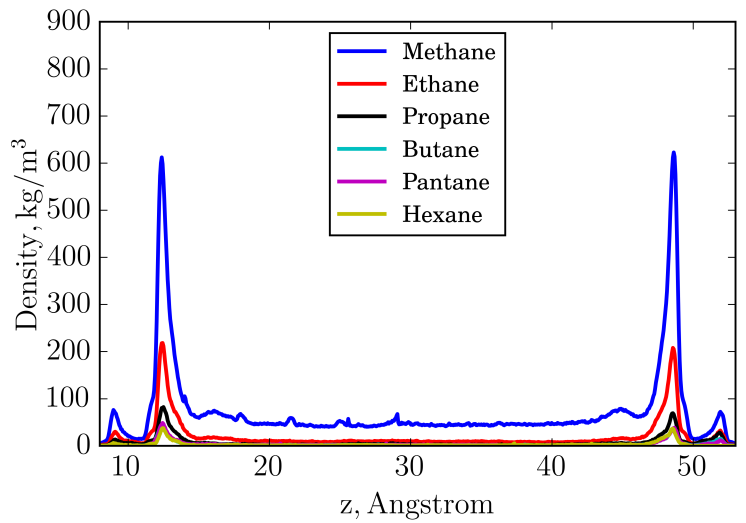

(b) $4 \mathrm{~nm}$

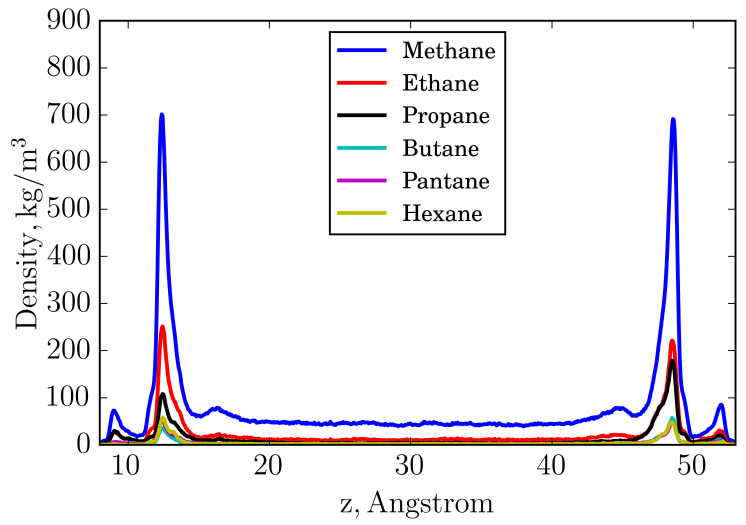

(d) $4 \mathrm{~nm}$

Figure 3.23: Density profiles of each gas component in the two samples in $2 \mathrm{~nm}$ and $4 \mathrm{~nm}$ channels.

where $S$ is selectivity, $x_{C_{6} H_{24}}$ is the mole fraction of hexane in adsorbed phase, and $y_{C_{6} H_{24}}$ represents 
the mole fraction of hexane in the bulk gas phase. Selectivity values higher than one show that the hexane molecules have more tendency to be adsorbed to walls compared to other gas components. Selectivity values of hexane over other gas components for both samples and channel sizes (plotted in Fig. 3.24 and listed in Table 4.14) decrease as the number of carbon atoms in the alkane chain increases. This means that heavier alkanes have higher tendency to be adsorbed to the wall compared to lighter alkanes. Based on these results, selectivity values are a function of molar composition. The differences in selectivity values between the samples decrease as channel size increases from 2 to $4 \mathrm{~nm}$.

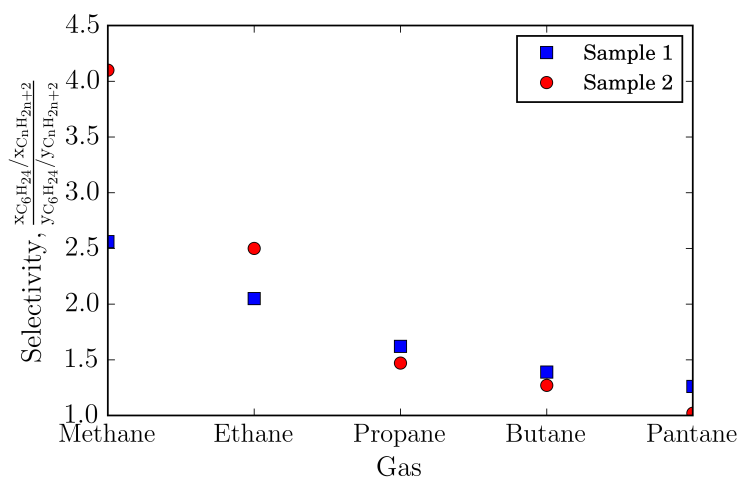

(a) $2 \mathrm{~nm}$

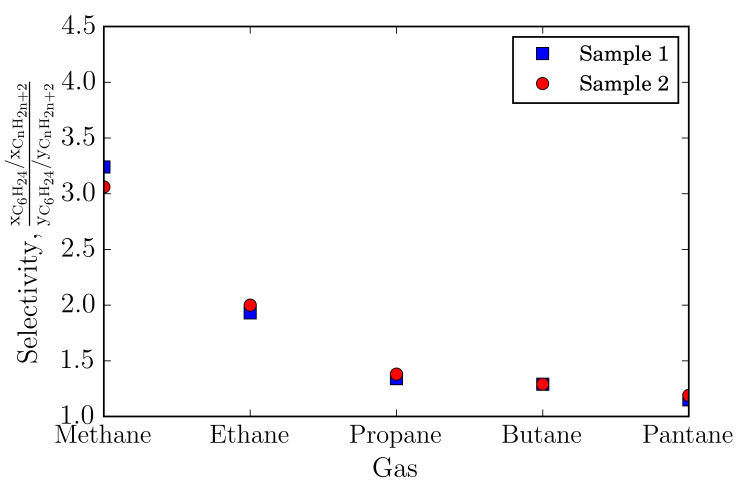

(b) $4 \mathrm{~nm}$

Figure 3.24: Selectivity of hexane over other gas components in (a) $2 \mathrm{~nm}$ and (b) $4 \mathrm{~nm}$ channels.

Table 3.7: Selectivities of hexane over other gas components for two samples used for simulations.

\begin{tabular}{|c|c|c|c|c|}
\hline \multirow{2}{*}{ Gas } & \multicolumn{4}{|c|}{ Selectivity } \\
\cline { 2 - 5 } & \multicolumn{2}{|c|}{ Sample 1} & \multicolumn{2}{c|}{ Sample 2} \\
\cline { 2 - 5 } & $2 \mathrm{~nm}$ & $4 \mathrm{~nm}$ & $2 \mathrm{~nm}$ & $4 \mathrm{~nm}$ \\
\hline \hline$S_{C_{6} H_{24} / C_{4}}$ & 2.56 & 3.24 & 4.1 & 3.06 \\
\hline$S_{C_{6} H_{24} / C_{2} H_{6}}$ & 2.05 & 1.93 & 2.53 & 1.99 \\
\hline$S_{C_{6} H_{24} / C_{3} H_{8}}$ & 1.61 & 1.34 & 1.47 & 1.38 \\
\hline$S_{C_{6} H_{24} / C_{4} H_{10}}$ & 1.39 & 1.29 & 1.27 & 1.29 \\
\hline$S_{C_{6} H_{24} / C_{5} H_{12}}$ & 1.23 & 1.15 & 1.02 & 1.19 \\
\hline
\end{tabular}




\section{Force Driven Flow}

Velocity Profiles Velocity profiles of sample 1 in 2 and $4 \mathrm{~nm}$ channels are plotted in Fig. 3.25. The black solid lines represent the location of the two-layered graphite sections and the black dashed lines represent locations of the peak densities in Fig. 3.22. As expected, the velocity increases as the applied force increases. A parabolic velocity profile is observed for all simulations. This is in contradiction with previous results on smooth surfaces in which a plug shaped flow profile were observed $[58,59]$. This might be due to the presence of heavier molecules and/or rough surface. Heavier molecules have higher tendency to be adsorbed walls and therefore, lower velocities are observed near the walls. Therefore, although the adsorbed phase transport are observed in recent studies, a rough surface may prevent or decrease the flow of these molecules. Additionally, for both channels, a sharp reduction of velocity is observed near the walls in between the dashed and solid lines. Similarly, in the $4 \mathrm{~nm}$ channel, parabolic profiles velocity are observed. The gas flows with low velocities near the wall in case of $4 \mathrm{~nm}$ channel, which shows that the adsorbed phase does not significantly contribute to the flow due to presence of rough surfaces.

Because the same number of molecules are used for both $2 \mathrm{~nm}$ and $4 \mathrm{~nm}$ channels, the pressure in the $4 \mathrm{~nm}$ channel is less than that in the $2 \mathrm{~nm}$ channel. Therefore, at the same external force, the velocities in $4 \mathrm{~nm}$ channel are higher than those of $2 \mathrm{~nm}$ channel due to higher Knudsen numbers in $4 \mathrm{~nm}$ channels. 


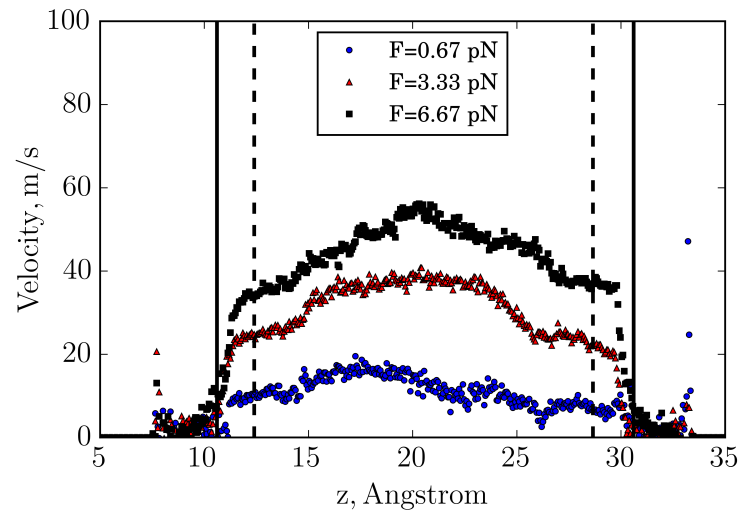

(a) $2 \mathrm{~nm}$

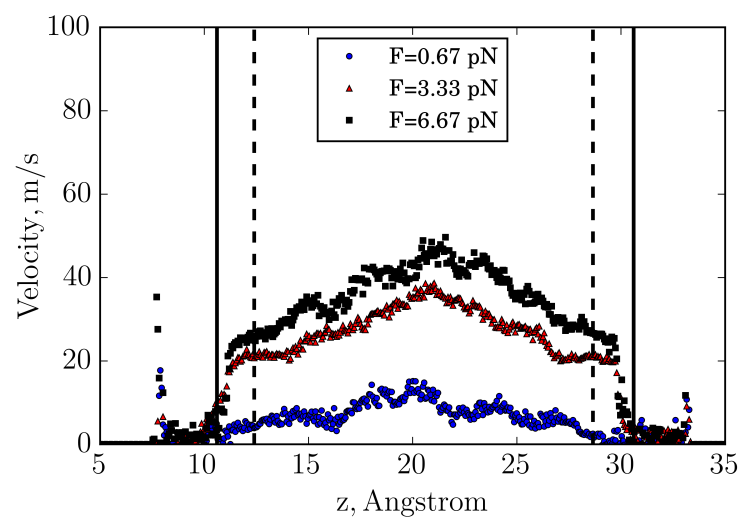

(c) $2 \mathrm{~nm}$

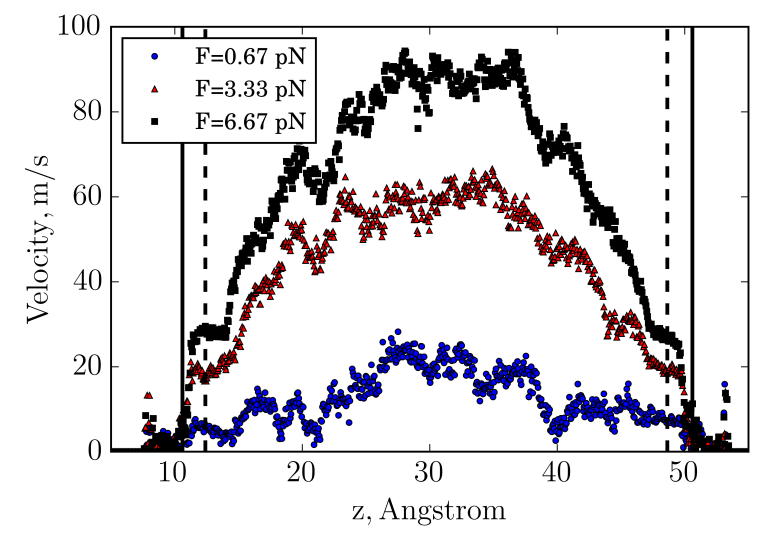

(b) $4 \mathrm{~nm}$

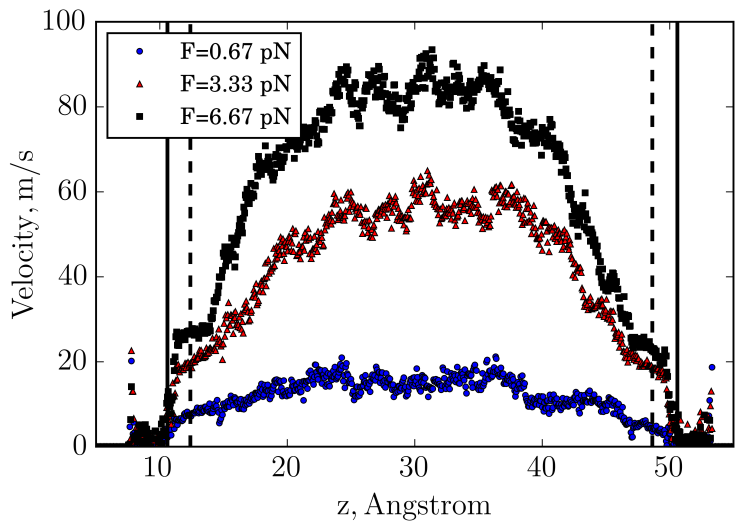

(d) $4 \mathrm{~nm}$

Figure 3.25: Velocity profiles of the gas samples in 2 and 4-nm channels. (a, b) Sample 1 and (c, d) Sample 2.

Molecular Flux The molecular flux of the gas samples for both channel heights are plotted in

Fig. 3.26. The molecular flux is calculated by multiplying the average gas density and velocity in channel. As discussed earlier, the pressure gradient across the channel is related to the external force applied, number of molecules to which the force is applied (inlet region), the length of the channel, and the area perpendicular to the flow (Equation 3.13). Number of the molecules at the inlet region is directly related to the gas density. Therefore, the molecular fluxes are plotted against a pressure gradient parameter defined as,

$$
\nabla P=\frac{\rho_{a v g} F}{A L} .
$$




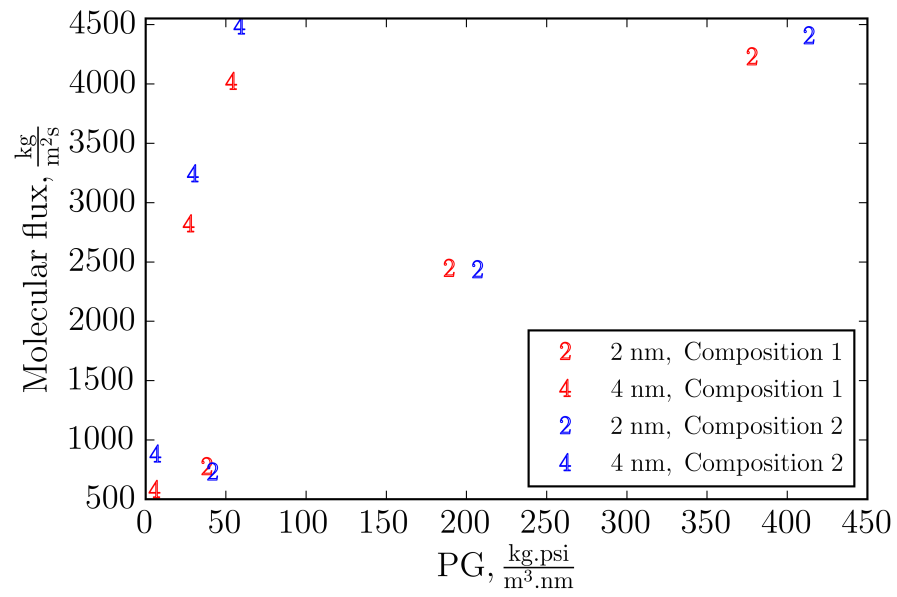

Figure 3.26: The molecular fluxes across the 2 and $4 \mathrm{~nm}$ channels for both samples.

For both gas samples, the molecular flux increases as the pressure gradient increases for both channel sizes. At the same pressure gradient, the molecular flux obtained from $4 \mathrm{~nm}$ channel is higher than that of $2 \mathrm{~nm}$ channel. It should be mentioned that the pressure of $2 \mathrm{~nm}$ channel is higher than $4 \mathrm{~nm}$ channel with the same composition. The rate of increase of the molecular flux with pressure gradient parameter is relatively linear and higher for $4 \mathrm{~nm}$ channel than $2 \mathrm{~nm}$ channel. The molecular flux of sample 1, which is lighter than sample 2, is slightly higher than sample $2 \mathrm{in} \mathrm{nm}$ channel. Therefore, the heavier components of sample 2 reduce its molecular flux in a $2 \mathrm{~nm}$ channel, while their effect in $4 \mathrm{~nm}$ channel is not significant.

Diffusion coefficients of each components for the gas samples are calculated using computed fluxes as [11],

$$
D_{t}=\frac{k_{B} T j}{\rho_{a v g} F}
$$

where $k_{B}$ is the Boltzmann constant, $T$ is the temperature, $j$ is the molecular flux, $\rho_{\text {avg }}$ is the average gas density in channel, and $F$ is the applied external force.

For both channel sizes, the diffusion coefficients decrease as the number of carbon atoms in the alkane chain increases (Fig. 3.27). In other words, components with larger molecular weights have lower diffusion coefficients. This might be due to the fact that the adsorption affinity of these molecules increase as their molar weight increases. Because these heavier molecules have lower diffusion coefficients than lighter components and they are adsorbed on the channel walls, low gas velocities are observed near the wall. With presence of surface roughness and heavy molecules, the lighter adsorbed gas molecules (such as methane and ethane) cannot flow with the same velocity as 
molecules in the middle of channel (Fig. 3.25) and therefore, the adsorbed phase does not contribute significantly to flow as were found in previous studies [47, 59].

Higher diffusion coefficients are obtained for the $4 \mathrm{~nm}$ channel compared to the $2 \mathrm{~nm}$ channel for each sample. Also, in the $2 \mathrm{~nm}$ channel, the diffusion coefficients of each component in Sample 1 is higher than those in sample 2. For the $4 \mathrm{~nm}$ channel, similar diffusion coefficients are found for both samples for components lighter than butane. Diffusion coefficients of the components heavier than butane in sample 2 are higher than in sample 1.

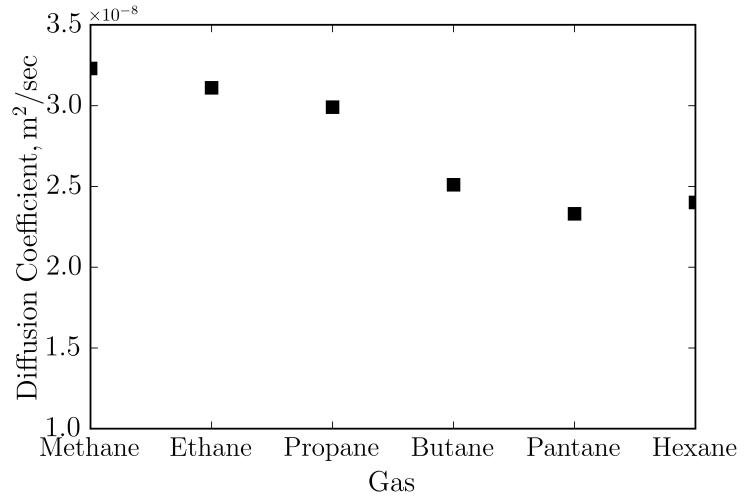

(a) $2 \mathrm{~nm}$, sample 1

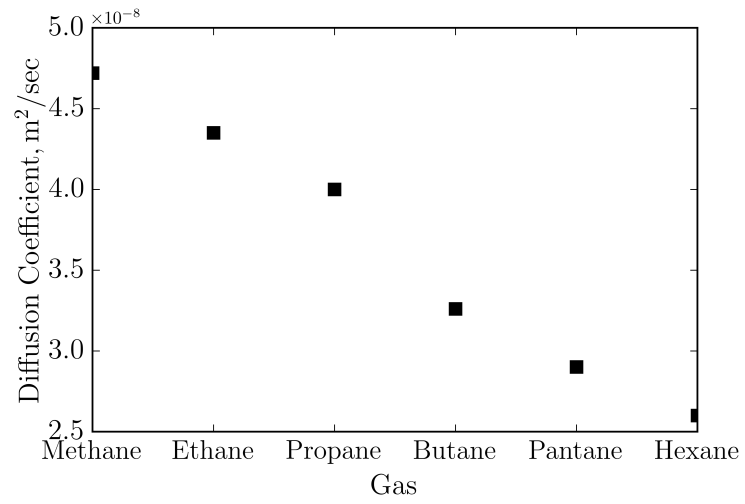

(c) $4 \mathrm{~nm}$, sample 1

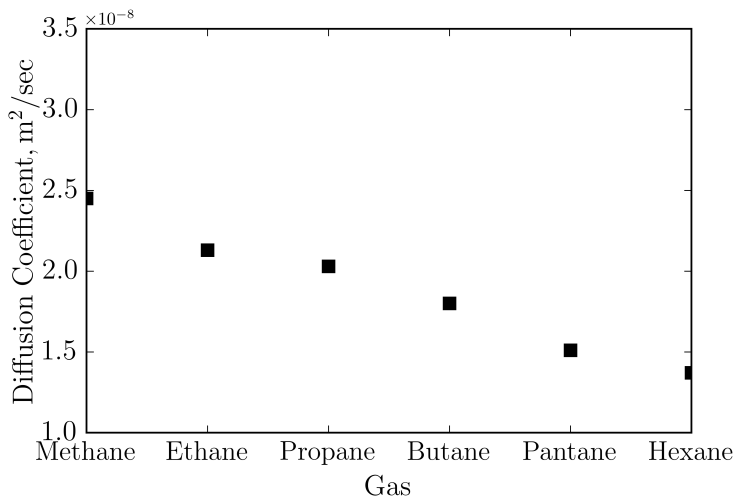

(b) $2 \mathrm{~nm}$, sample 2

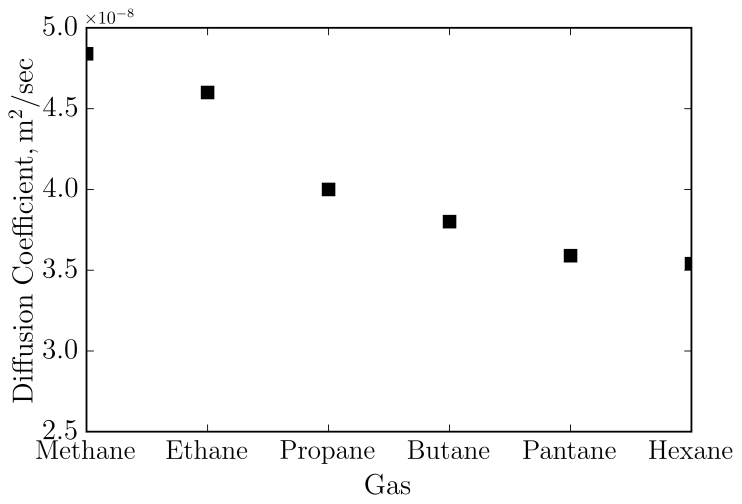

(d) $4 \mathrm{~nm}$, sample 2

Figure 3.27: Diffusion coefficients of each gas component in the gas samples for 2- and 4-nm channels.

\subsubsection{Conclusions}

Multicomponent gas transport and adsorption in carbon-based organic channels with rough surfaces are investigated by performing non-equilibrium molecular dynamics simulations for two gas samples. 
Table 3.8: Diffusion coefficients of different gas components in the gas samples.

\begin{tabular}{|c|c|c|c|c|}
\hline \multirow{3}{*}{ Gas } & \multicolumn{3}{|c|}{ Diffusion Coefficient $\left(10^{-8} \mathrm{~m}^{2} / \mathrm{s}\right)$} \\
\cline { 2 - 5 } & \multicolumn{2}{|c|}{ Sample 1 } & \multicolumn{2}{|c|}{ Sample 2} \\
\cline { 2 - 5 } & $2 \mathrm{~nm}$ & $4 \mathrm{~nm}$ & $2 \mathrm{~nm}$ & $4 \mathrm{~nm}$ \\
\hline \hline$D_{C H_{4}}$ & 3.23 & 4.72 & 2.45 & 4.84 \\
\hline$D_{C_{2} H_{6}}$ & 3.11 & 4.35 & 2.13 & 4.6 \\
\hline$D_{C_{3} H_{8}}$ & 2.99 & 4 & 2.03 & 4 \\
\hline$D_{C_{4} H_{10}}$ & 2.51 & 3.26 & 1.8 & 3.8 \\
\hline$D_{C_{5} H_{12}}$ & 2.33 & 2.9 & 1.51 & 3.59 \\
\hline$D_{C_{6} H_{14}}$ & 2.4 & 2.6 & 1.37 & 3.54 \\
\hline
\end{tabular}

Large portions of the heavier gas components are adsorbed to walls. Selectivity values of hexane, which is the heaviest gas component, compared to other gas components are higher than 1, which shows that it has the highest adsorption tendency. Therefore, in a organic nanopores of shale, the heavier components may remain in reservoir in adsorbed phase while the lighter components are produced.

In contrary to previous studies on single component gases flow in carbon-based smooth conduits, plug-shaped velocity profiles are not observed and the gas molecules velocities near the wall are smaller than other parts of channel. Lower velocities near the wall may be due to presence of rough surfaces and components heavier than methane.

Pressure in the $4 \mathrm{~nm}$ channel is lower than that in the $2 \mathrm{~nm}$ channel. Furthermore, at the same pressure gradient, the molecular flux values obtained in $4 \mathrm{~nm}$ channel is higher than those in the 2 $\mathrm{nm}$ channel. The rate of increase of molecular flux with pressure gradient parameter is higher for 4 nm channel.

The molecular flux of light composition sample is slightly higher than that of heavy composition sample in the $2 \mathrm{~nm}$ channel due to its higher composition of light molecules. Therefore, the heavier components reduce the molecular flux in the $2 \mathrm{~nm}$ channel, while their effect in the $4 \mathrm{~nm}$ channel is not significant. Furthermore, for both channels, diffusion coefficients decrease as the number of carbon atoms in the alkane chain increases (components become heavier). 


\subsection{DCV-GCMD Simulations of Pure Gases in Smooth Or- ganic Nanocapillaries}

Transport properties of fluids, such as density and viscosity, in confined systems may be significantly different from their nominal values (unconfined properties).

As far as viscosity, several authors have presented Several viscosity models have been reported that can predict the viscosity of the fluids under confinement by relating them to the nominal gas viscosity values and Knudsen number $(K n)[5,16,18,52,84,96,108]$,

$$
\frac{\mu_{\text {actual }}}{\mu_{\text {nominal }}}=f(K n)
$$

However, these models are developed for inorganic pores and the gas behavior is organic pores are different due to adsorption of gas molecules to surface of the pore. due to the interaction forces between the organic molecules at the surfaces of the pores, heterogeneous fluid distributions exist in these pores, that can cause local changes in density and viscosity of the fluids [68, 73].

In this work, we compare the transport of gases with high adsorbing capacity (methane and argon) with the ones with low adsorbing capacity (neon) in carbon-based organic capillaries. Monte Carlo simulation are performed within Grand Canonical ensemble to predict the adsorption properties (excess and absolute) of investigated gases. DCV-GCMD simulations are then carried out in capillaries with $2,4,6$, and $8 \mathrm{~nm}$ diameter at different pressures and pressure gradients. Furthermore, density, mass flux, and velocity profiles of the gases are compared. Effect of system geometry (pore diameter) and average pore pressure on diffusion coefficients in organic nanopores is studied. The predictions made by the Knudsen diffusion model are compared with DCV-GCMD results. Furthermore, the viscosities of argon, methane, and neon are determined and analyzed based on Karniadakis et al. [52] viscosity model.

\subsubsection{Problem Setup}

Two graphite capillaries are created using Moltemplate molecular builder software [46] with diameters of 2 and $4 \mathrm{~nm}$. The walls of the capillaries are made with three layers of graphite. The distance between concentric carbon capillaries are $0.335 \mathrm{~nm}$. The distance between two adjacent carbon 
atoms in the same carbon capillary is 0.142 . The length for these capillaries are chosen to be 40 and $80 \mathrm{~nm}$ for 2 and $4 \mathrm{~nm}$ capillaries (Length to Diameter ratio of 20), respectively. The capillaries are placed between two bulk reservoirs with high $(\mathrm{H})$ and low $(\mathrm{L})$ pressures. The pressures of the bulk reservoirs are kept constant by insertion and deletion of molecules. Temperature of the system is set to $300 K$ using the Nose-Hoover thermostat. Two graphite walls are placed at the ends of the bulk reservoirs to avoid their direct contact because of the periodic boundary condition that is applied in $z$ direction. Furthermore, the lengths of the reservoirs are selected to be 2 and $4 \mathrm{~nm}$, respectively. Additionally, two hollowed walls are placed at both ends of the capillaries to guide all the gas molecules toward the capillaries.

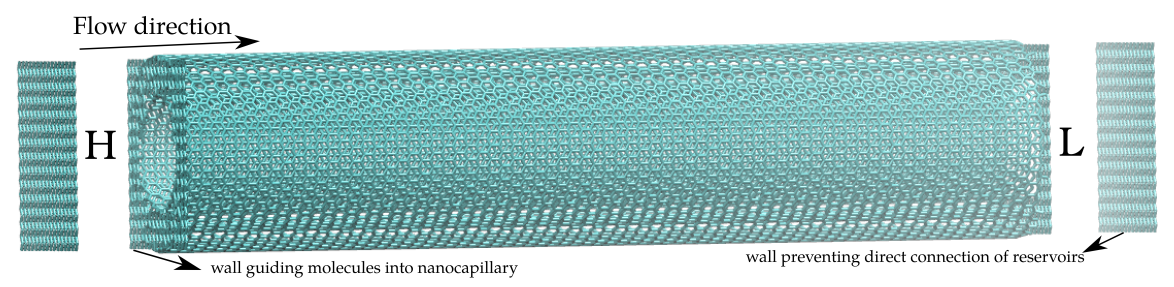

Figure 3.28: Simulation setup for the $2 \mathrm{~nm}$ capillary. The capillary length is $40 \mathrm{~nm}$. "H" and "L" represent the high and low pressure reservoirs, respectively.

\subsubsection{Results and Discussion}

\section{Adsorption isotherms}

In this subsection, absolute and excess adsorption of the gases are studied by performing GCMC simulations. Absolute adsorption is total number of molecules contained in the pore per unit mass of solid [100]. Excess adsorption is the actual amount of gas present minus the amount of gas that would be present in the same space at prevailing bulk density. These simulations are performed at a fugacity range of 10 to $285 \mathrm{~atm}$ for argon, 10 to $222 \mathrm{~atm}$ for methane, and 10 to $374 \mathrm{~atm}$ for neon to investigate their adsorption behavior in the capillaries. These fugacity ranges translate to a pressure range of 10 to $330 \mathrm{~atm}$ for each gas. The amount of gas in bulk phase (unconfined) is defined as the amount of gas in the absence of pore walls at specified temperature and pressure.

Density of the gas molecules with and without confinement are shown in Fig. 3.29. In unconfined situations, density of gases increase as pressure (and fugacity). For inert gases, such as neon and argon, this increase is relatively linear. However, for methane, which is not an ideal gas, this increase only linear for low pressures (below $50 \mathrm{~atm}$ ). As pressures increase, the increase becomes 
nonlinear. Absolute adsorption quantities increase as the fugacity increases and tend to saturate at higher fugacities for argon and methane (dashed lines in Fig. 3.29). In the $2 \mathrm{~nm}$ capillary, methane and argon have similar absolute adsorptions and are distinctly different from the ones for neon (Fig. 3.29a). In the $4 \mathrm{~nm}$ capillary, absolute adsorption of methane is higher than argon and neon for entire pressure range (Fig. 3.29b). Absolute adsorption values for methane ranges from 1.5 to $7.6 \mathrm{~mol} / \mathrm{kg}$ as fugacity increases from 10 to $220 \mathrm{~atm}$ compared to those for argon for which the adsorption values change from 0.5 to $5.3 \mathrm{~mol} / \mathrm{kg}$ for the same fugacity range. For both capillaries, neon has lower adsorption quantities compared to methane and argon, which demonstrates that it is a less adsorbing gas compared to methane and argon.

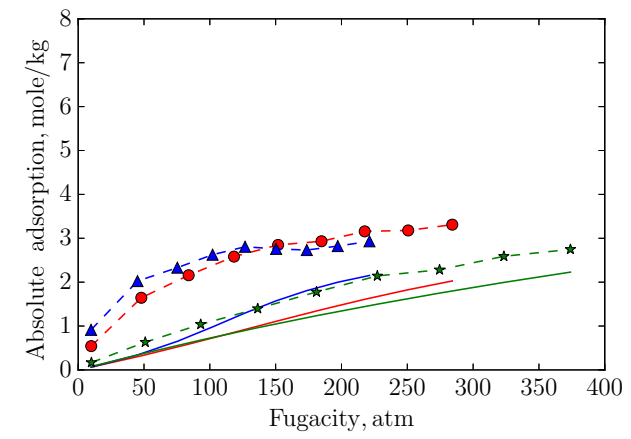

(a) $2 \mathrm{~nm}$

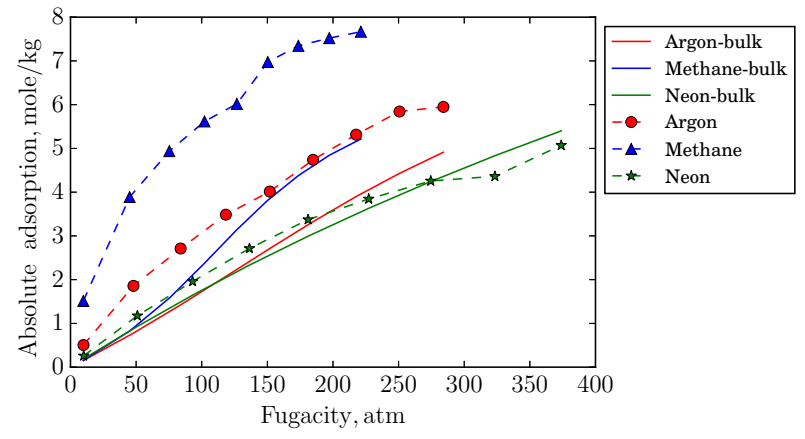

(b) $4 \mathrm{~nm}$

Figure 3.29: Absolute adsorptions for the (a) $2 \mathrm{~nm}$ and (b) $4 \mathrm{~nm}$ capillaries.

Excess adsorption values are small at $10 \mathrm{~atm}$ for all gases because the bulk and absolute adsorption values are similar for both capillaries (at low pressures all gases behave as ideal gases) (Fig. 3.30). As pressure (fugacity) increases, the excess loading reaches a maximum above which the loadings decrease. In $2 \mathrm{~nm}$ capillary, all gases reach a maximum adsorption (at an optimum fugacity) after which the adsorption quantities decrease. In the $4 \mathrm{~nm}$ capillary, the excess adsorption quantities for methane are distinctively higher than those for argon and neon while in $2 \mathrm{~nm}$ capillary, argon and methane excess values are in the same range. In $4 \mathrm{~nm}$ capillary, adsorption values for methane and argon reach as high as 3.35 and $1.41 \mathrm{~mol} / \mathrm{kg}$, respectively. The difference in isotherms of two capillaries is because the amount of adsorbed gas is directly scales with capillary surface area. The surface area of $4 \mathrm{~nm}$ capillary is 8 times greater than the $2 \mathrm{~nm}$ capillary. Neon excess adsorption values are less than the other two gases and range from -0.07 to $0.5 \mathrm{~mol} / \mathrm{kg}$ for $2 \mathrm{~nm}$ capillary and -0.4 to 0.3 for $4 \mathrm{~nm}$ capillary. Therefore, the neon density under confinement is similar to its density without confinement (excess quantities are close to zero). 


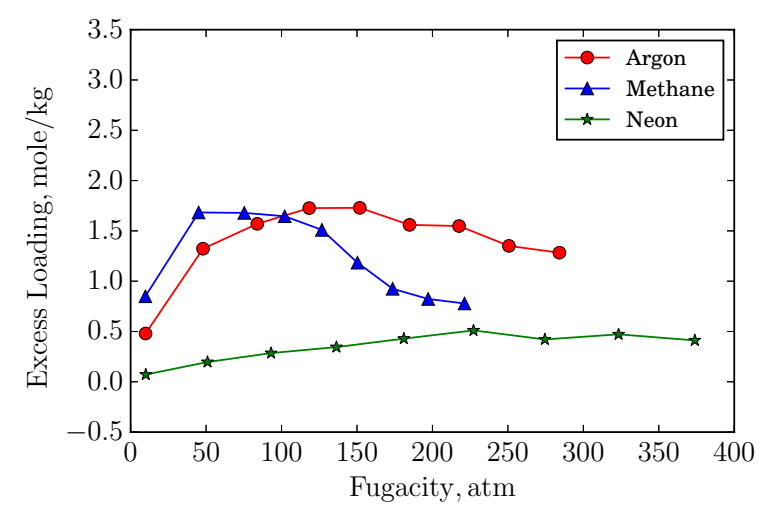

(a) $2 \mathrm{~nm}$

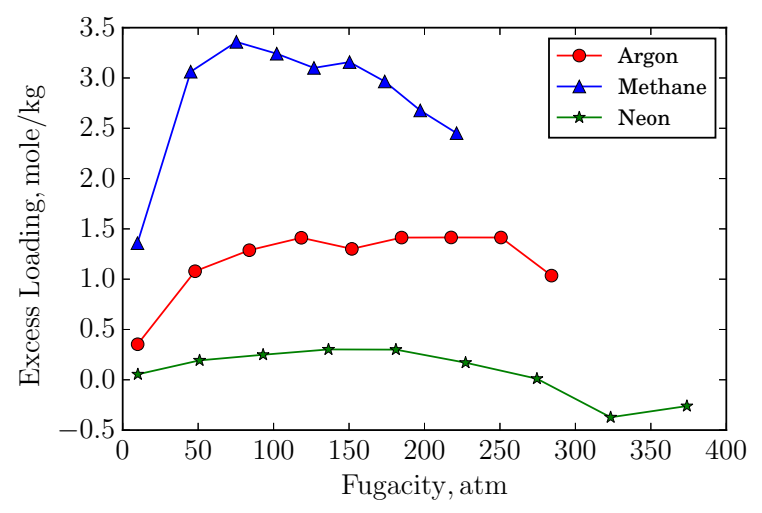

(b) $4 \mathrm{~nm}$

Figure 3.30: Excess adsorptions for a (a) $2 \mathrm{~nm}$ and (b) $4 \mathrm{~nm}$ capillaries.

\subsubsection{Chemical Potential Driven Flow}

In this section, the normalized velocity (velocity divided by the average velocity across the capillary) profiles of the gases across the capillary are presented. The normalized velocity profiles at the same pressure for different pressure gradients are the same, which shows that the flow regime falls in the linear response regime [4].

DCV-GCMD simulations are performed at three mean pressures of 20, 50, and $90 \mathrm{~atm}$. The mean pressure is the average pressure of the two reservoirs. The length of the capillaries are selected to be $40,80,120$, and $160 \mathrm{~nm}$ for tubes with $2,4,6$, and $8 \mathrm{~nm}$ diameters, respectively, to have a fully established flow and avoid any connections between the two reservoirs. Several pressure gradients are applied at each pressure. The density and normalized gas velocity profiles of argon, methane, and neon at different pressures are plotted in Figs. 3.31, 3.33 and 3.35, respectively. The mass fluxes are also computed and compared with Knudsen diffusion predictions in Figs. 3.32, 3.34, and 3.36.

\section{A. Argon}

Density of argon at the wall increases as pressure increases in all capillaries (Fig. 3.31). For instance, for the $2 \mathrm{~nm}$ capillary, argon density at the wall increases from 1763 to $3549 \mathrm{~kg} / \mathrm{m}^{3}$ as pressure increases from 20 to $100 \mathrm{~atm}$. Because the adsorption isotherm is not a linear function of the pressure, the rate of increase of gas density at the wall decreases as pressure increases. 
Normalized velocity values range from 0.95 to 1.05 , which demonstrate the formation of approximately plug-shaped flow profiles for argon in the 2 and $4 \mathrm{~nm}$ capillaries. As tube size increases, argon molecules tend to have a lower velocities at the vicinity of the wall than at the middle of the capillary. The normalized velocity of 6 and $8 \mathrm{~nm}$ capillaries range from 0.8 to 1.15 at $20 \mathrm{~atm}$. However, at $100 \mathrm{~atm}$, the normalized velocity ranges from 0.89 to 1.11 . Therefore, a-plug shaped flow profile exists even with larger capillary sizes. In other words, gas molecules in both bulk phase and adsorbed phase have similar velocities for all the examined pressure gradients. One of the reasons for this behavior might be the smooth structure-less geometry of the capillary walls.
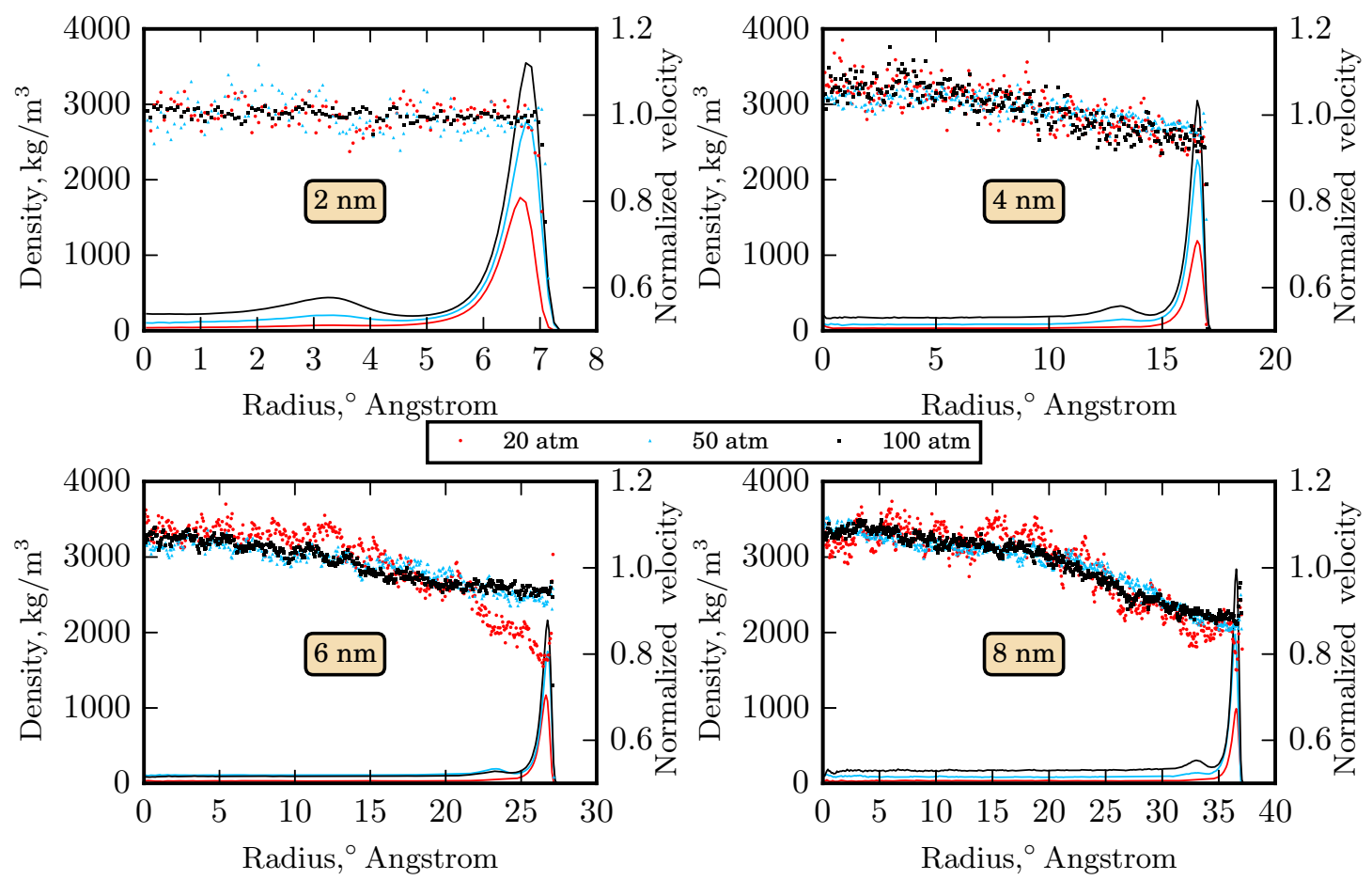

Figure 3.31: Density and normalized velocity profiles of Argon.

For all the pressures, mass flux values are linearly change with pressure gradients for both capillary diameters (Fig. 3.32). The molecular fluxes computed from DCV-GCMD simulations are at least one order of magnitude higher than those estimated by the Knudsen diffusion. This is due to the contribution of adsorbed phase to the flow. These results are consistent with the results published by Holt et al. [42]. The simulations also demonstrate that at lower pressures, the molecular fluxes are slightly higher than those at high pressures for the same pressure gradients. 


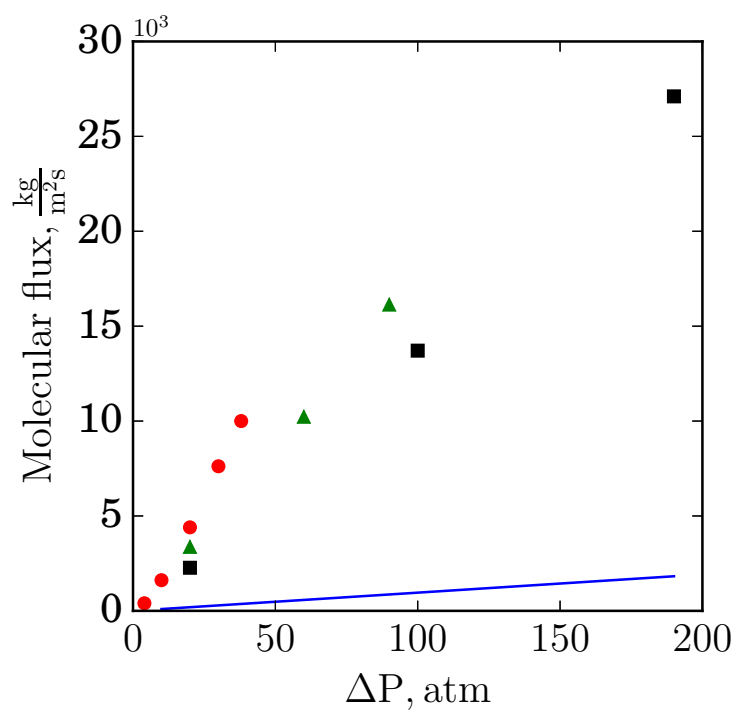

(a) $2 \mathrm{~nm}$

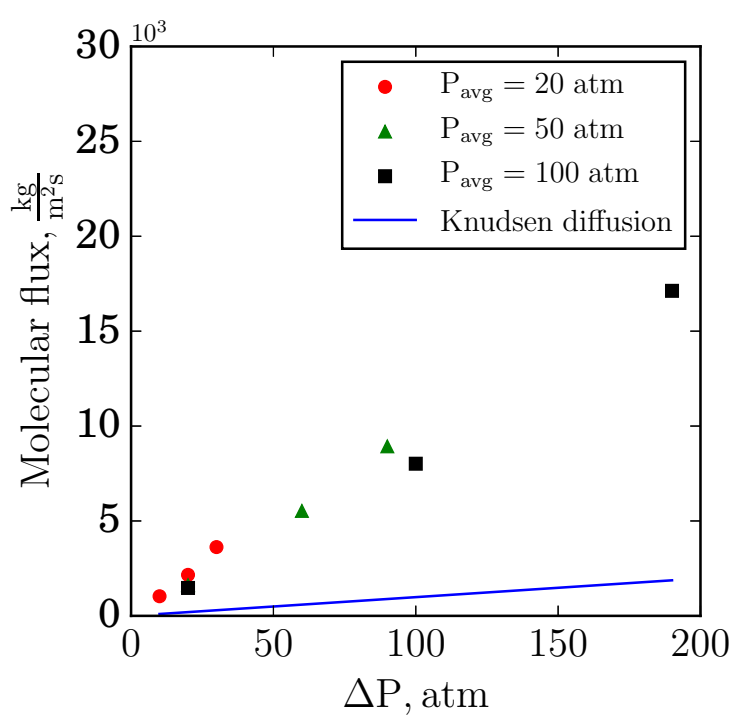

(b) $4 \mathrm{~nm}$

Figure 3.32: A comparison of calculated mass flux with the ones predicted by Knudsen diffusion. (a) $2 \mathrm{~nm}$ (b) $4 \mathrm{~nm}$

\section{B. Methane}

Similar to argon, as the pressure increases, methane density at the wall increases (Fig. 3.33). For instance, for the $2 \mathrm{~nm}$ capillary, the gas density at wall increases from 1104 to $1625 \mathrm{~kg} / \mathrm{m}^{3}$ for a pressure range of 20 to $100 \mathrm{~atm}$.

The normalized velocity at different pressures ranges from 0.95 to 1.08 for the $2 \mathrm{~nm}$ capillary. As the capillary diameter increases, the normalized velocity range increases. For instance, in the $8 \mathrm{~nm}$ capillary, normalized velocity values range from 0.75 to 1.1 . As the pressure increases, the velocity profiles become less concave until they reach plug-shapes velocity profiles. The normalized velocity values are close to 1 that one can say that the methane molecules at the wall flow with a similar velocity as the gas molecules in the bulk phase.

Computed molecular fluxes for methane are at least one order of magnitude larger than those predicted by Knudsen diffusion (Fig. 3.34). Similar to argon, as pressure decreases, the slope of the plot increases. 

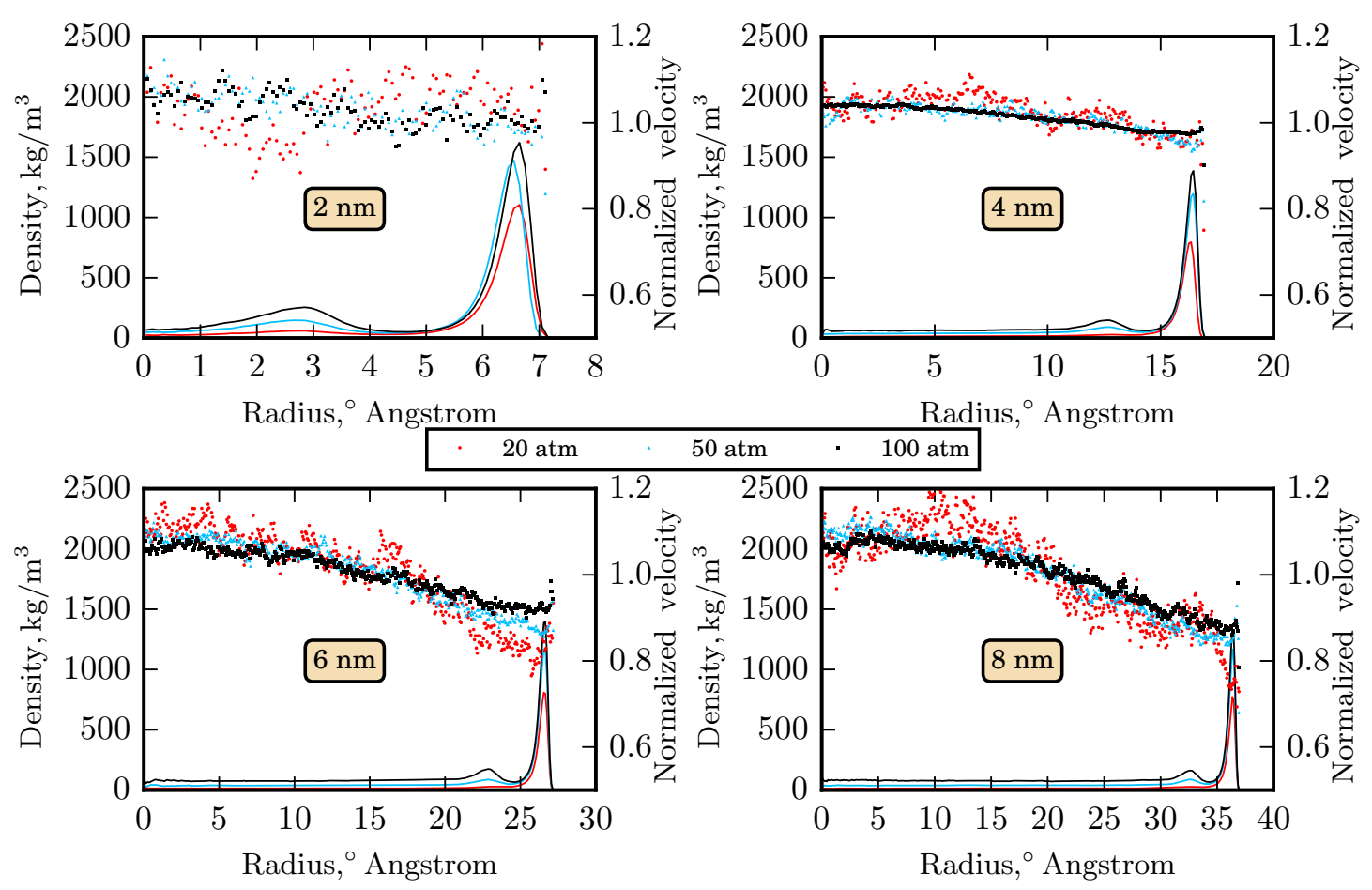

Figure 3.33: Density and normalized velocity profiles of Methane.

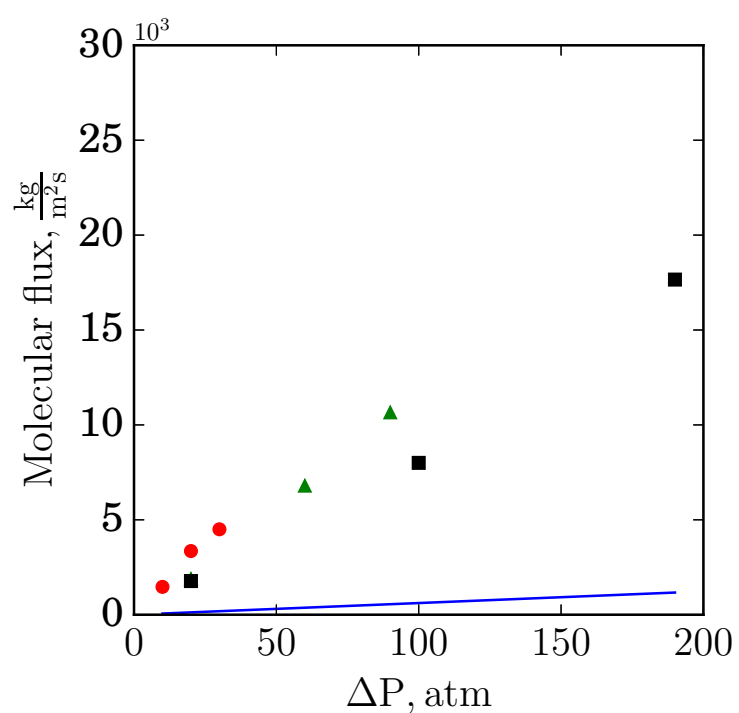

(a) $2 \mathrm{~nm}$

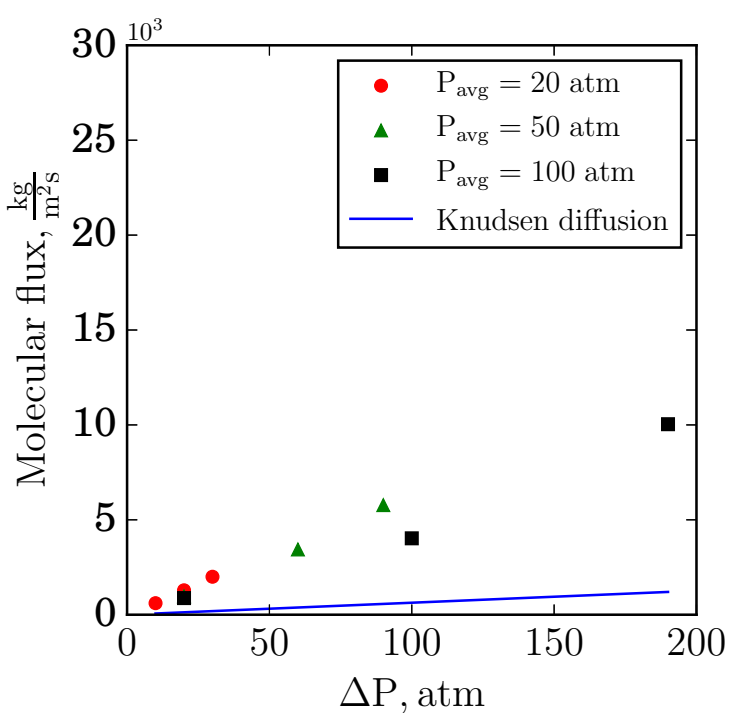

(b) $4 \mathrm{~nm}$

Figure 3.34: A comparison of computed mass flux for methane with the ones predicted by Knudsen diffusion. (a) $2 \mathrm{~nm}$ (b) $4 \mathrm{~nm}$ 


\section{Neon}

Although the neon density is higher near the wall, total neon gas amount in capillary is almost equal to its bulk amount leading (approximately zero excess adsorption) as shown in Fig. 3.30a and 3.30b.

The velocities of neon molecules are similar across the tube and a plug-shaped flow profile is observed similar to those for argon and methane (Fig. 3.35). Comparing argon, methane, and neon normalized velocities, it can be observed that methane has lowest velocity near the wall. Argon's normalized velocity is slightly higher than methane and lower than neon. For example, for the $8 \mathrm{~nm}$ capillary at $20 \mathrm{~atm}$ pressure, the normalized velocities of methane, argon, and neon at wall are $0.75,0.8$, and 0.85 , respectively.
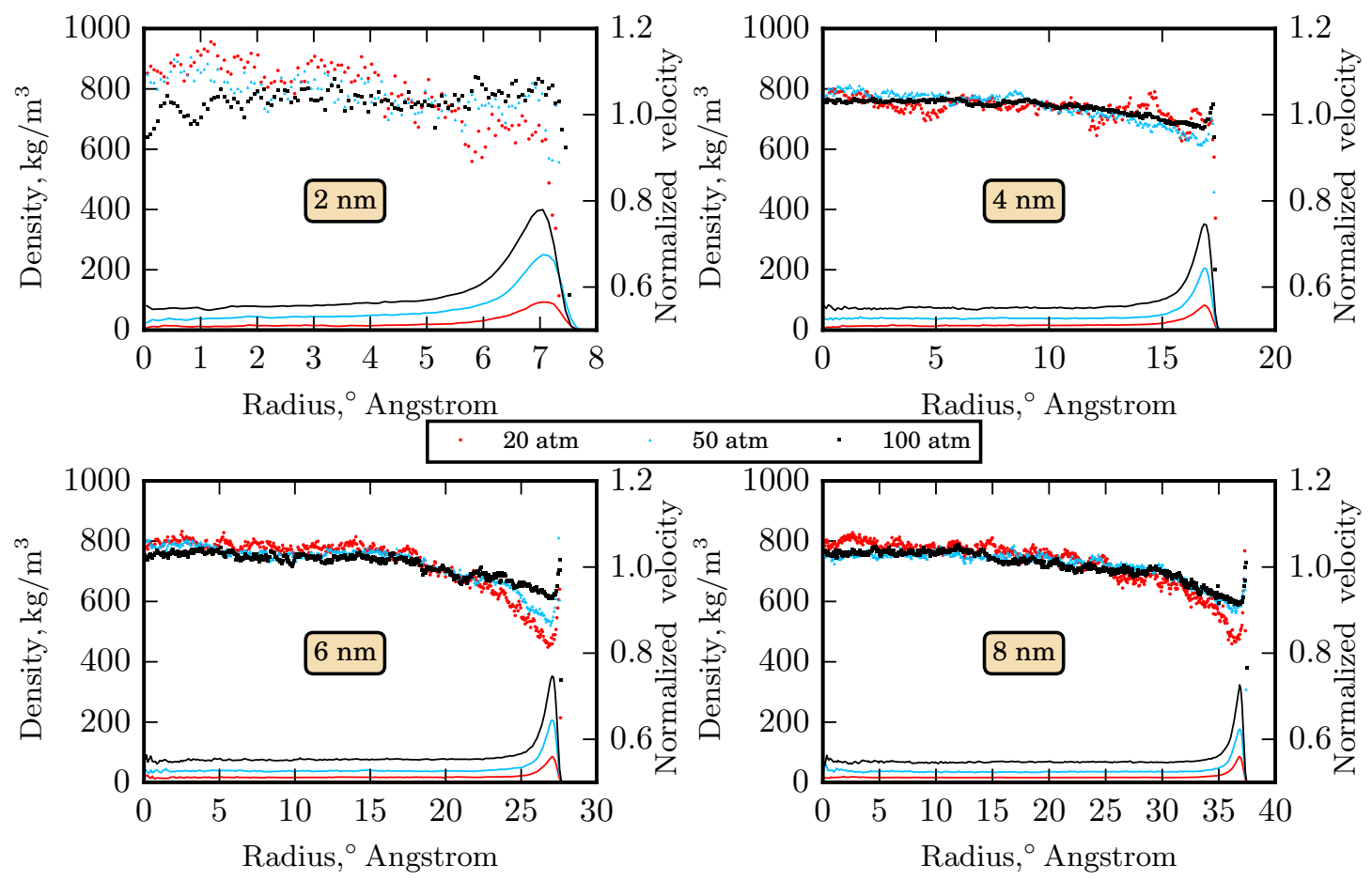

Figure 3.35: Density and normalized velocity profiles of Neon.

Computed molecular fluxes are roughly 5 times higher than those of predicted with Knudsen diffusion model (Fig. 3.36). 


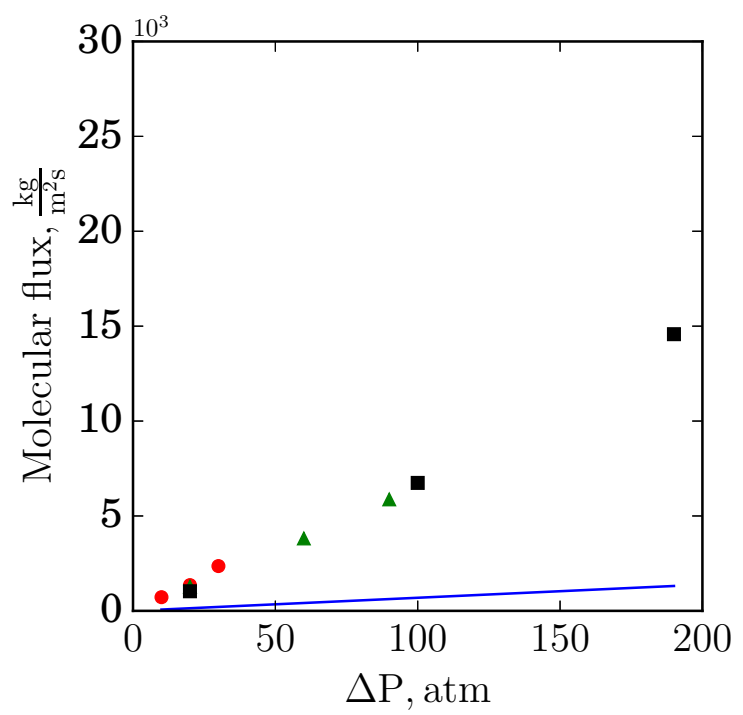

(a) $2 \mathrm{~nm}$

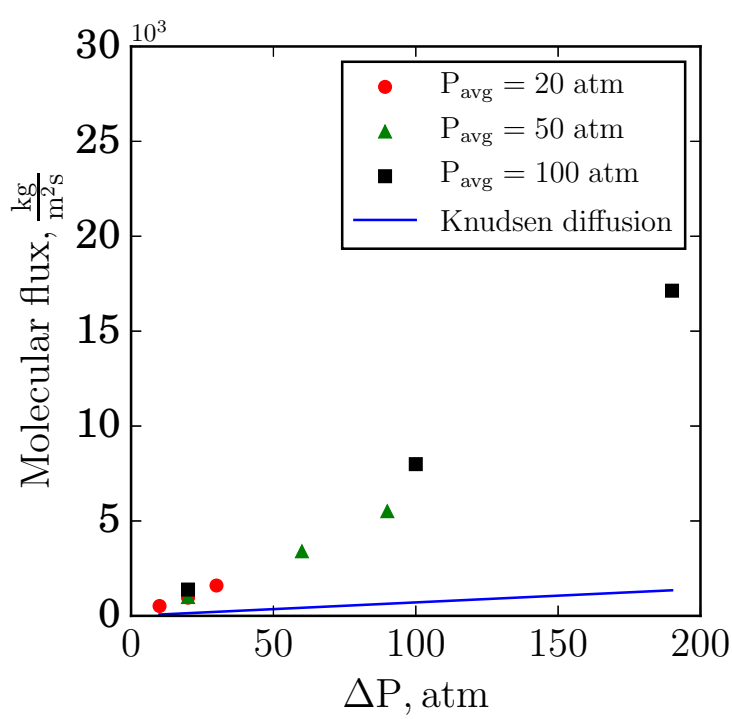

(b) $4 \mathrm{~nm}$

Figure 3.36: A comparison of COMPUTED mass flux values for neon with the ones predicted by Knudsen diffusion. (a) $2 \mathrm{~nm}$ (b) $4 \mathrm{~nm}$

The lower methane normalized velocities at the wall can be attributed to its gas-wall strength of potential interaction. Fig. 3.37 demonstrate the Lennard-Jones strength of potential of these three gases. Methane have the highest strength $(\epsilon)$ while neon have the lowest gas-wall strength of potential. Therefore, neon molecules would flow with higher velocities near the wall compared with argon and neon.

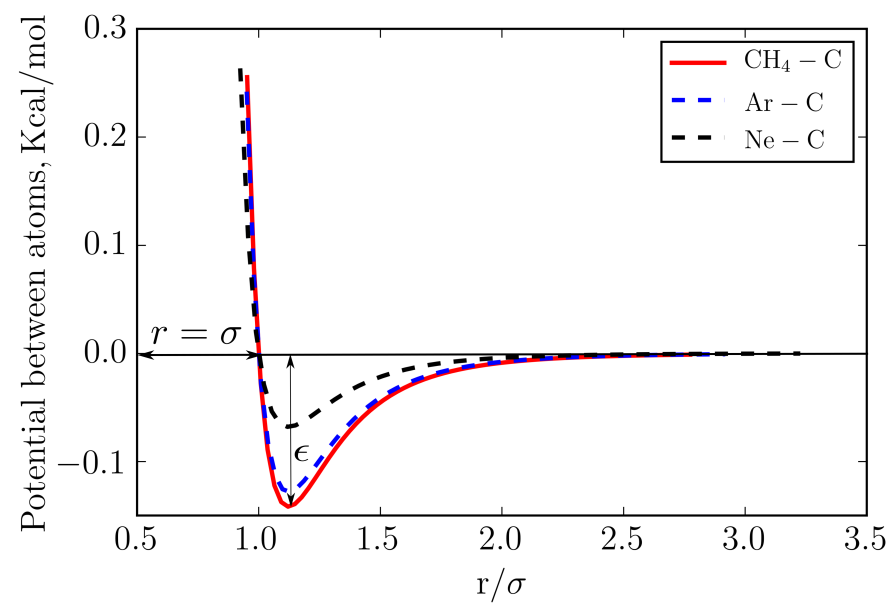

Figure 3.37: Lennard-Jones potential of studied gases.

The molecular fluxes determined from DCV-GCMD simulations are at least one order of magnitude 
higher than those predicted from Knudsen diffusion model for both argon and methane (Fig. 3.32 and 3.34). This underestimation is lower for neon in comparison with argon and methane.

\subsubsection{Comparison of R13 Model with Molecular Simulations}

In this section, the molecular simulation results for velocity profiles are compared with the analytical velocities developed using R13 model [56]. The tube surface-gas molecules interaction are accounted for using the tangential momentum accommodation coefficient (TMAC) in this model. In our previous works $[55,56]$, we validated $\mathrm{R} 13$ results using the TMAC of unity, meaning the interactions are fully diffusive. Due to smooth nature of the geometries in this study, we intend to find the TMAC values for the gases in the 2, 4, 6, and $8 \mathrm{~nm}$ capillaries.

The molecular simulation velocity profiles are compared with R13 model. TMAC values are found by fitting the molecular simulation results with those of R13 model for each gas and capillaries. In the presented results (Figs. 3.38, 3.39 ,3.41, and 3.40), the solid lines represent the model result and points show results for the molecular simulations.

For methane and argon and capillary diameters $(2,4,6$, and $8 \mathrm{~nm})$, the TMAC values in R13 model are altered until good with the simulation results is reached. The velocity profile resulted from R13 model with fully diffusive (TMAC $=1$, green curves) and fully specular (TMAC=0, blue curves) boundary conditions are also plotted in the same figures. It is demonstrated that the fully diffusive boundary condition tend to underestimate the velocity values for the simulated geometries, while the specular boundary overestimate the velocity values.

The Knusden numbers and pressure under which molecular simulations are performed are listed in Table 3.9. For argon and methane in both tubes, as pressure increases (Knudsen decreases), TMAC values increase. In other words, at lower pressures, the wall-gas interactions are more specular-like compared to higher pressures. Neon simulations cover a higher range of Knudsen numbers (up to 3.6) for the same pressure range due to its smaller molecular size of neon compared to methane and argon. It should be pointed out the R13 model is validated for Knudsen values up to unity [55]. Therefore, the TMAC values found for Knudsen number larger than unity are not used in this work. For each has type, the TMAC values do not follow the same trend line for 2 and $4 \mathrm{~nm}$ capillaries, which might be due the different adsorption properties (Fig. 3.3). To account for the adsorption properties in the two tubes, the TMAC values are multiplied by adsorption surface coverage, $\theta$ 


\begin{tabular}{lllrr}
\hline Gas & Size & Pressure & Kn & TMAC \\
\hline Methane & 2 & 20 & 0.292 & 0.038 \\
& & 50 & 0.221 & 0.055 \\
& & 100 & 0.185 & 0.085 \\
& 4 & 20 & 0.306 & 0.135 \\
& & 50 & 0.188 & 0.170 \\
& & 100 & 0.155 & 0.176 \\
& 6 & 20 & 0.261 & 0.155 \\
& & 50 & 0.149 & 0.405 \\
& & 100 & 0.103 & 0.340 \\
& & 20 & 0.150 & 0.120 \\
Argon & & 50 & 0.132 & 0.360 \\
& & 100 & 0.089 & 0.410 \\
\hline & & 20 & 0.507 & 0.050 \\
& 4 & 100 & 0.313 & 0.060 \\
& & 20 & 0.232 & 0.075 \\
& & 50 & 0.278 & 0.125 \\
& 6 & 20 & 0.181 & 0.185 \\
& & 50 & 0.561 & 0.235 \\
& & 100 & 0.152 & 0.300 \\
& 8 & 20 & 0.961 & 0.4280 \\
& & 50 & 0.426 & 0.630 \\
& & 100 & 0.241 & 0.520 \\
\hline
\end{tabular}

Table 3.9: The Knudsen numbers and pressures under which the molecular simulations are performed.

(Fig. 3.42). Doing this, for both capillaries, the products of TMAC and surface coverage for argon and methane fall in the same trend. 


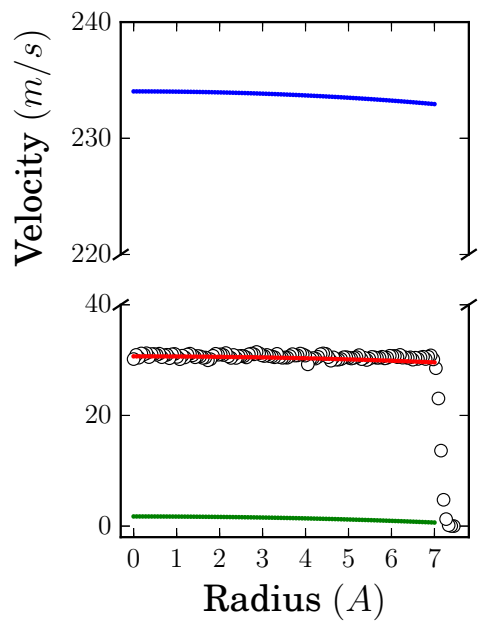

(a) $\mathrm{Kn}=0.23$

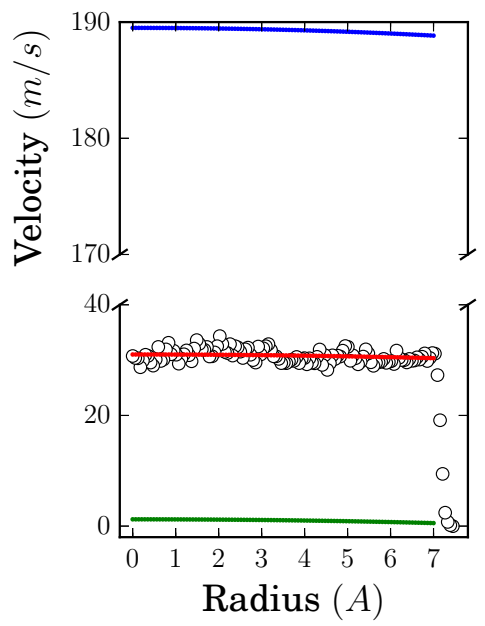

(b) $\mathrm{Kn}=0.51$
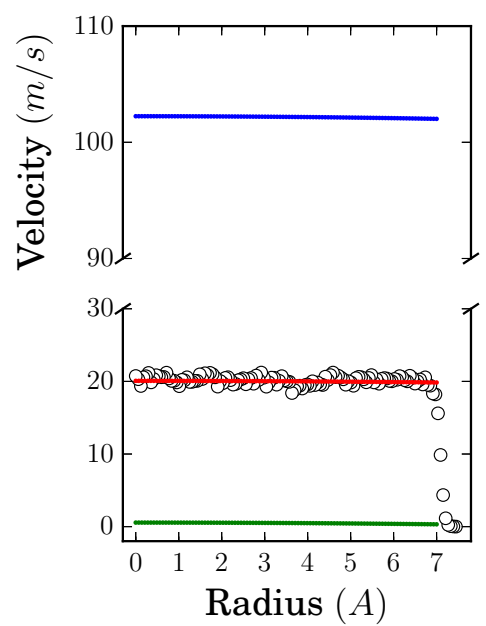

(c) $\mathrm{Kn}=1.72$

Figure 3.38: Comparison of the simulation results with R13 model for argon in the $2 \mathrm{~nm}$ capillary (a) $\mathrm{P}_{\text {avg }}=100 \mathrm{~atm}, \Delta \mathrm{P}=100 \mathrm{~atm}$, (b) $\mathrm{P}_{\text {avg }}=50 \mathrm{~atm}, \Delta \mathrm{P}=60 \mathrm{~atm}$, and (c) $\mathrm{P}_{\text {avg }}=20 \mathrm{~atm}, \Delta \mathrm{P}$ $=20 \mathrm{~atm}$

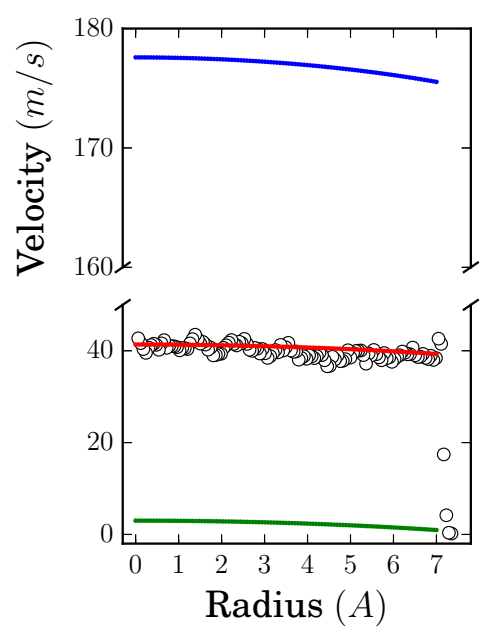

(a) $\mathrm{Kn}=0.19$

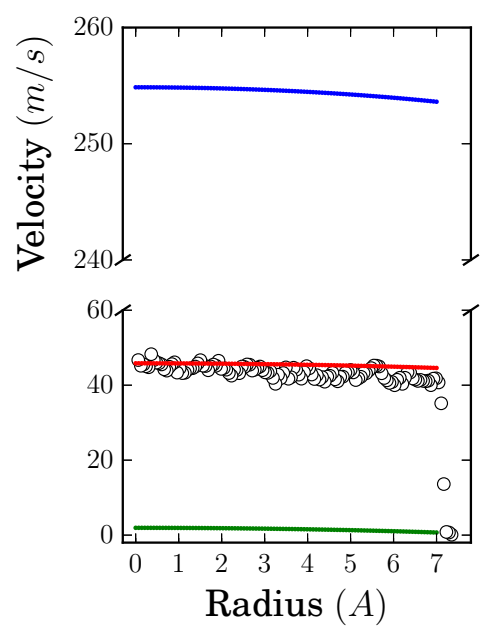

(b) $\mathrm{Kn}=0.22$

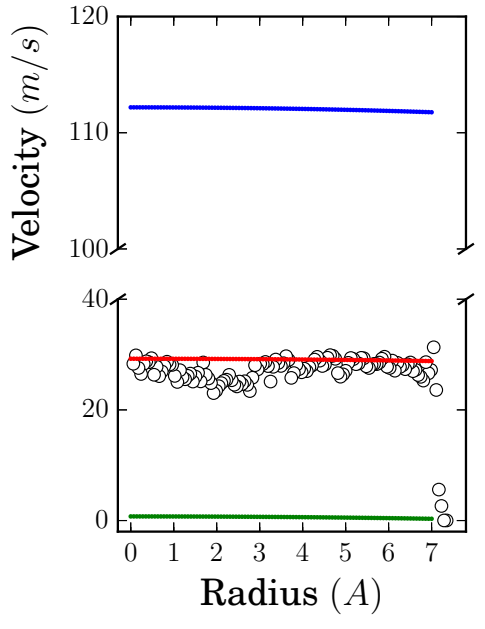

(c) $\mathrm{Kn}=0.29$

Figure 3.39: Comparison of the simulation results with $\mathrm{R} 13$ model for methane in the $2 \mathrm{~nm}$ capillary (a) $\mathrm{P}_{\text {avg }}=100$ atm, $\Delta \mathrm{P}=100$ atm, (b) $\mathrm{P}_{\text {avg }}=50$ atm, $\Delta \mathrm{P}=60$ atm, and (c) $\mathrm{P}_{\text {avg }}=20$ atm, $\Delta \mathrm{P}$ $=20 \mathrm{~atm}$ 


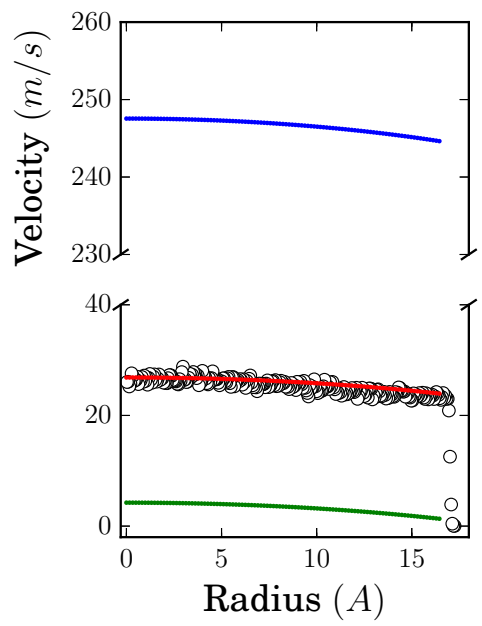

(a) $\mathrm{Kn}=0.18$
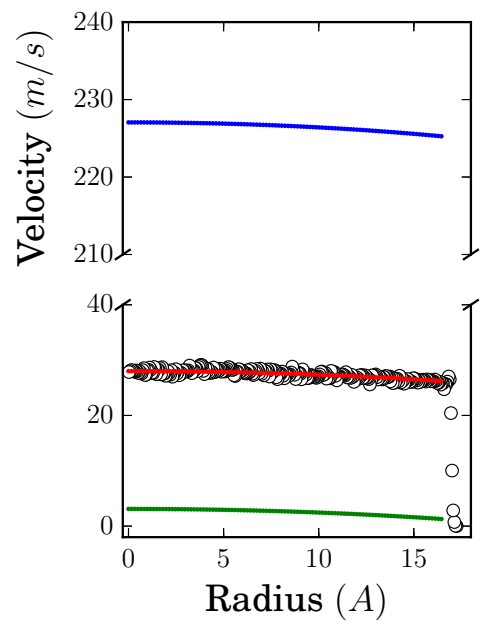

(b) $\mathrm{Kn}=0.28$
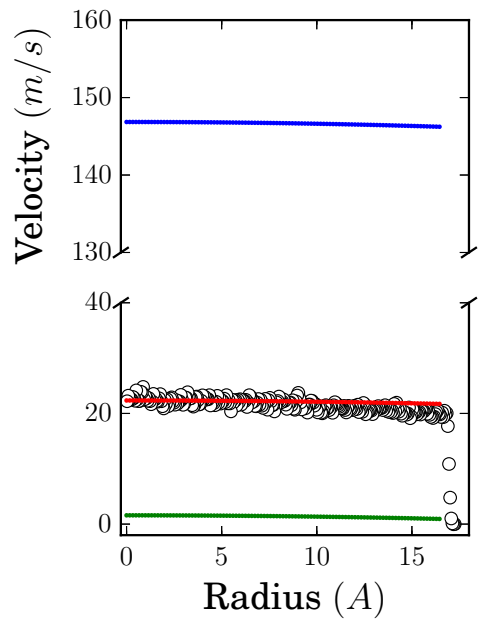

(c) $\mathrm{Kn}=0.54$

Figure 3.40: Comparison of the simulation results with R13 model for argon in the $4 \mathrm{~nm}$ capillary (a) $\mathrm{P}_{\text {avg }}=100 \mathrm{~atm}, \Delta \mathrm{P}=100 \mathrm{~atm}$, (b) $\mathrm{P}_{\text {avg }}=50 \mathrm{~atm}, \Delta \mathrm{P}=60 \mathrm{~atm}$, and (c) $\mathrm{P}_{\text {avg }}=20 \mathrm{~atm}, \Delta \mathrm{P}$ $=20 \mathrm{~atm}$

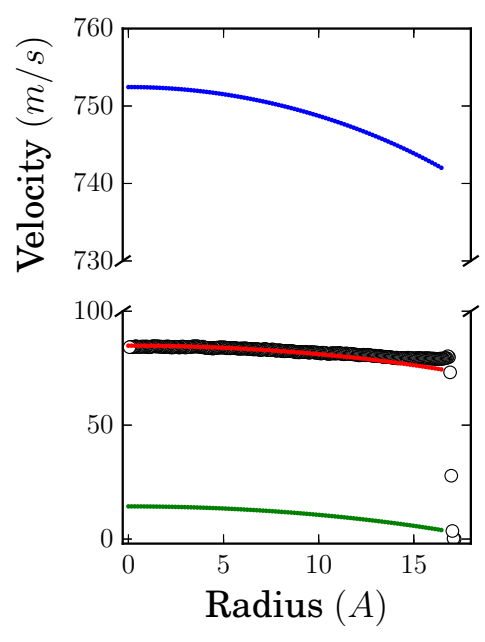

(a) $\mathrm{Kn}=0.16$

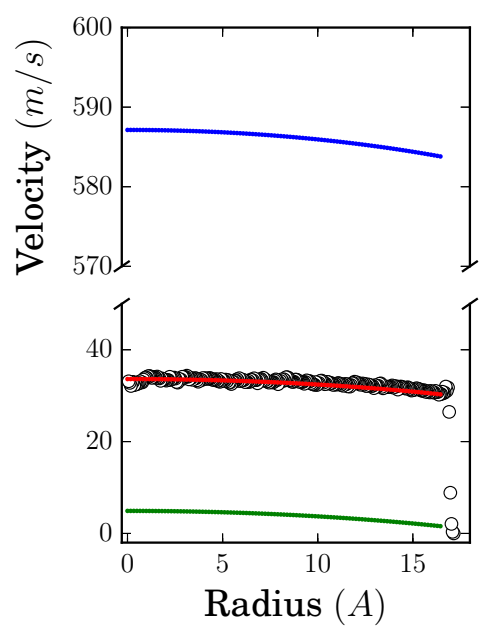

(b) $\mathrm{Kn}=0.19$

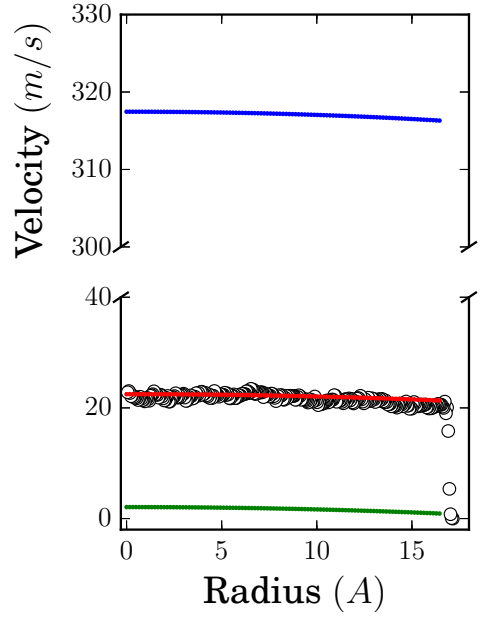

(c) $\mathrm{Kn}=0.31$

Figure 3.41: Comparison of the simulation results with R13 model for methane in the $4 \mathrm{~nm}$ capillary (a) $\mathrm{P}_{\text {avg }}=100$ atm, $\Delta \mathrm{P}=100$ atm, (b) $\mathrm{P}_{\text {avg }}=50$ atm, $\Delta \mathrm{P}=60$ atm, and (c) $\mathrm{P}_{\text {avg }}=20$ atm, $\Delta \mathrm{P}$ $=20 \mathrm{~atm}$ 


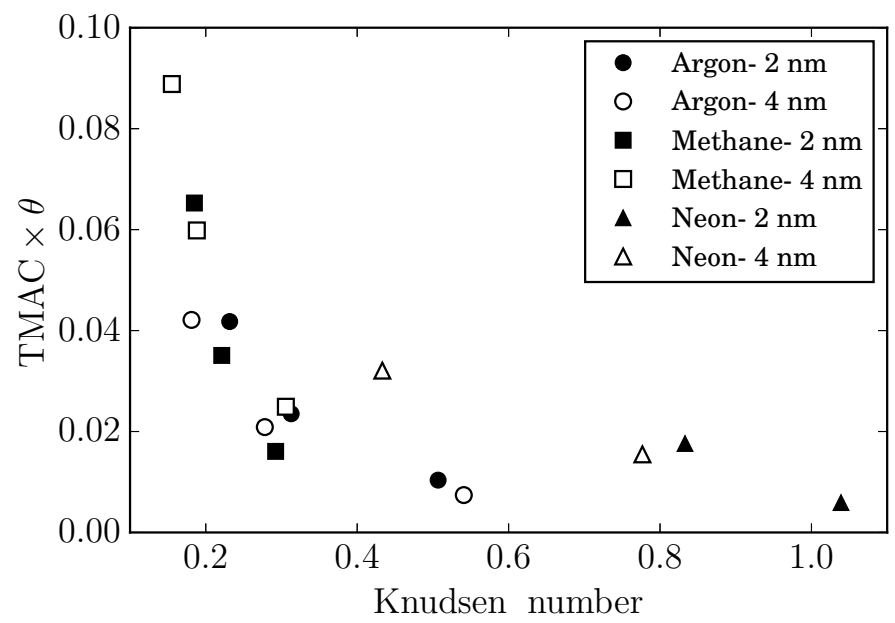

Figure 3.42: The product of TMAC and adsorption surface coverage, $\theta$, as a function of Knudsen number.

\subsubsection{Diffusion Coefficients}

In this study, transport diffusion coefficients for methane and argon are determined. The Langmuir isotherms are fitted to absolute adsorption of argon and methane for the 2 and $4 \mathrm{~nm}$ capillaries and thermodynamic factors are determined. Finally, corrected diffusivity coefficients are determined using Eq. 3.18.

$$
D_{T}=D_{c} \frac{\partial \ln (f)}{\partial \ln (c)}
$$

Both transport and corrected diffusivity coefficients for argon and methane decrease as pressure (fugacity) increases (Fig. 3.43). The corrected diffusivity coefficients are smaller than the transport ones because the thermodynamic factors are larger than one. The thermodynamics factors are equal to one when for unconfined systems density increases linearly with pressure. This is the case for the ideal gases. For confined systems, the thermodynamic factor is larger than one. Therefore, corrected and transport diffusion coefficients become identical in unconfined systems at infinite dilution limit. The difference between the transport and corrected diffusion coefficients increase as pressure increases because the absolute adsorption amount increases with pressure.

The differences between the transport and corrected transport diffusivity values are smaller for the 4 $n m$ capillary compared with those for the $2 \mathrm{~nm}$ one (Fig. 3.43). This shows that the thermodynamic factor in the $2 \mathrm{~nm}$ capillary is larger than that in the $4 \mathrm{~nm}$ capillary. The thermodynamic factor of the $2 \mathrm{~nm}$ capillaries are higher than those of the $4 \mathrm{~nm}$ for argon and methane (Fig. 3.44). At 
smaller capillary diameters, for the same pressure, molecules collide with capillary walls with increasingly higher probability compared with intermolecular collisions. Therefore, the capillary wall can significantly influence the transport diffusion coefficient as the capillary size reduces. Furthermore, transport diffusion coefficient is influenced by thermodynamic factor as indicated in Eq. 3.18.

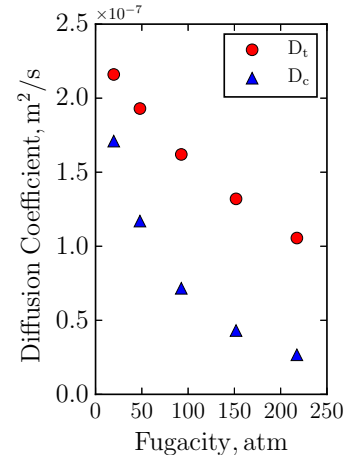

(a) Argon, $2 \mathrm{~nm}$

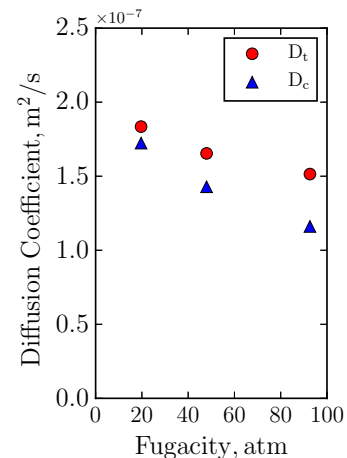

(b) Argon, $4 \mathrm{~nm}$

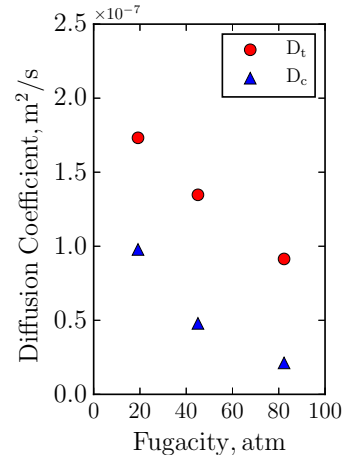

(c) Methane, $2 \mathrm{~nm}$

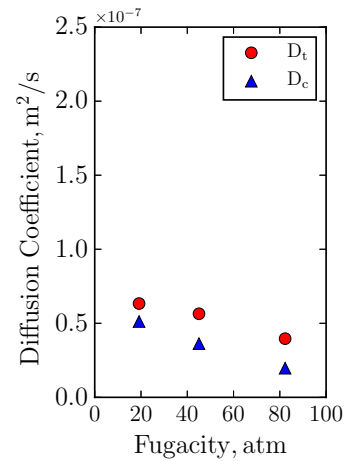

(d) Methane, $4 \mathrm{~nm}$

Figure 3.43: Transport and corrected diffusivity coefficients for argon and methane. (a) $2 \mathrm{~nm}$ (b) 4 nm.

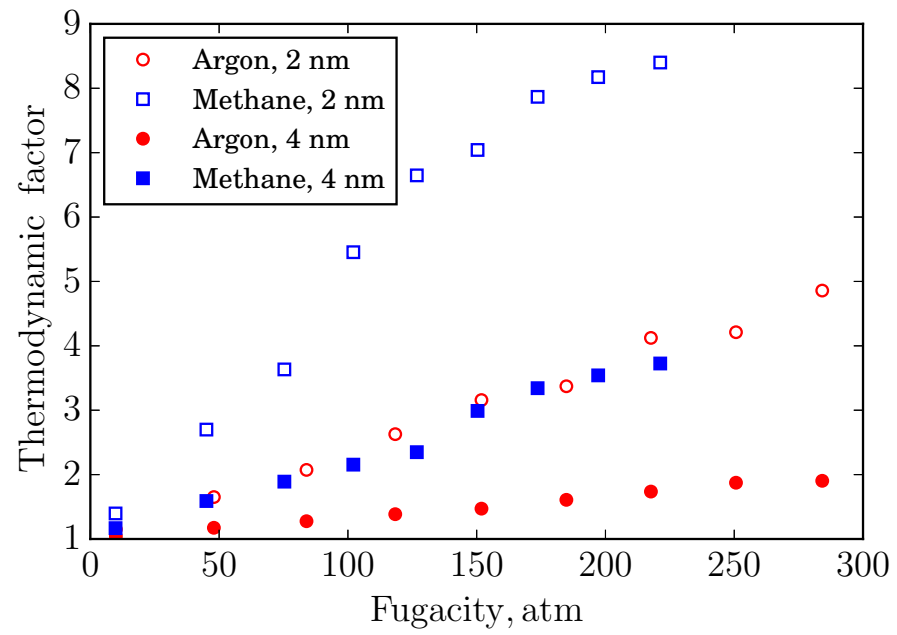

Figure 3.44: Thermodynamic factors for argon and methane in the 2 and $4 \mathrm{~nm}$ capillaries.

\subsubsection{Viscosity}

In order to determine the viscosity of the gases an empirical equation developed by Hippler et al. [41] which relates the gas molecules self-diffusion coefficient to viscosity. In order to apply this equation 
for dilute gases, it was modified by [28] and is casted as,

$$
\frac{T^{*}}{\mu^{*} D^{*}}=2 \pi \sigma^{*}\left[1-\exp \left(-\frac{64 \rho^{*}}{15 \sigma^{*}} \Omega^{(1,1)} \Omega^{(2,2)}\right)\right]
$$

where,

$$
\begin{aligned}
& \sigma^{*}=\frac{5.02}{2 \pi \rho^{*}}, \quad \rho^{*}=\rho \sigma^{3}, \quad T^{*}=\frac{k_{B} T}{\epsilon}, \quad \mu^{*}=\frac{\mu \sigma^{2}}{(m \epsilon)^{0.5}}, \quad D^{*}=\frac{D}{\sigma}\left(\frac{m}{\epsilon}\right)^{0.5} \\
& \Omega^{(1,1)}=\frac{1.06}{\left(T^{*}\right)^{0.16}}+\frac{0.19}{\exp \left(0.48 T^{*}\right)}+\frac{1.04}{\exp \left(1.53 T^{*}\right)}+\frac{1.76}{\exp \left(3.89 T^{*}\right)} \\
& \Omega^{(2,2)}=\frac{1.16}{\left(T^{*}\right)^{0.15}}+\frac{0.52}{\exp \left(0.77 T^{*}\right)}+\frac{2.16}{\exp \left(2.44 T^{*}\right)}-6.43 \times 10^{-4}\left(T^{*}\right)^{0.15} \\
& \times \sin \left(18.03\left(T^{*}\right)^{-0.77}-7.27\right) .
\end{aligned}
$$

In this equation, $\rho$ is number density, $\sigma$ is molecular diameter, $\epsilon$ is Lennard-Jones strength of potential, $m$ is molecular mass, $T$ is temperature, $k_{B}$ is Boltzmann constant, $\mu$ is viscosity, and $D$ is self-diffusion coefficient. The modified Hippler et al. [41] equation relates the viscosity of the gas to self-diffusion coefficient. Equilibrium molecular dynamics (EMD) simulations are usually carried out to the determine the self-diffusivity coefficients. Self-diffusion, $D_{s}$, is defined as the mean square displacement of molecules over time,

$$
D_{s}=\frac{1}{6 N} \lim _{t \rightarrow \infty} \frac{d}{d t}\left\langle\sum_{i=1}^{n}\left[\vec{r}_{i}(t)-\vec{r}_{i}(0)\right]^{2}\right\rangle
$$

where $N$ is the number of molecules and $\overrightarrow{r_{i}}(t)$ is the location of the $i^{t h}$ molecule at time $t$. The angular bracket represents the ensemble average quantity. Self-diffusion coefficients for argon, methane, and neon are determined at different pressures by performing EMD simulations.

Self-diffusion coefficients for all gases decrease as pressure increases for the 2 and $4 \mathrm{~nm}$ capillaries (Figs. 3.45). For the simulated pressure range, the computed self-diffusion coefficients for adsorbing gases (argon and methane) are higher in the $4 \mathrm{~nm}$ capillaries than those of $2 \mathrm{~nm}$. However, the difference between viscosities of 2 and $4 \mathrm{~nm}$ capillaries reduces as pressure increases.

For neon, the self-diffusion coefficients are approximately one order of magnitude larger than those in argon and methane. This might be due to the fact that for neon, which has a lower heat of adsorption and molecular weight compared to argon and methane, the molecules in the capillaries have more freedom to move and are less influenced by the force-field of the carbon molecules on the pore walls as shown in Fig. 3.37. The computed values for neon in the 2 and $4 \mathrm{~nm}$ capillaries are 
approximately the same except at $10 \mathrm{~atm}$, where the self-diffusion coefficients for the $4 \mathrm{~nm}$ are larger than those in the $2 \mathrm{~nm}$. This shows that the effect of adsorption on the self-diffusion coefficients is minimal for neon.

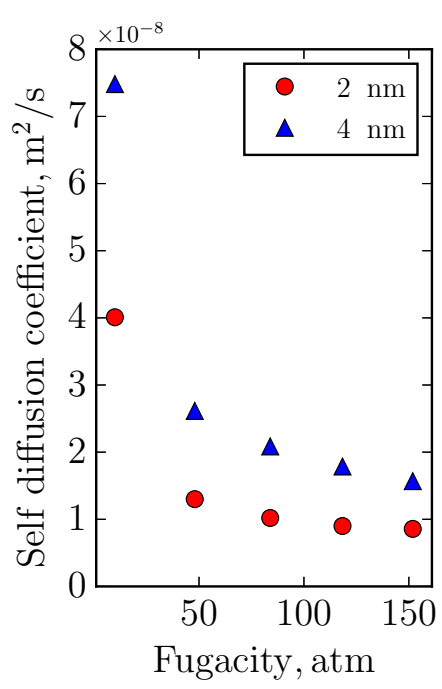

(a) Argon

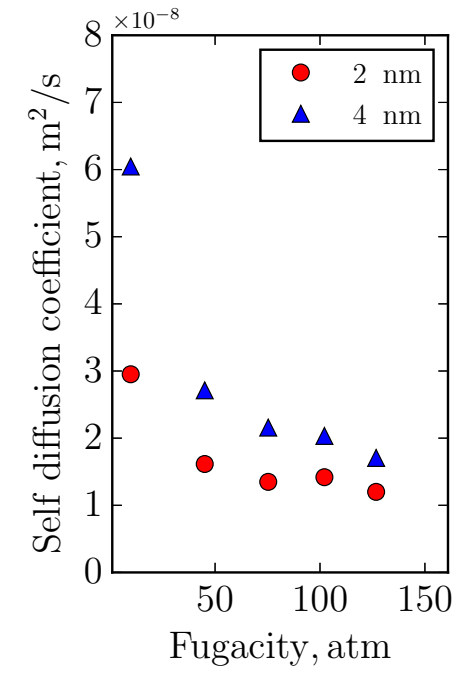

(b) Methane

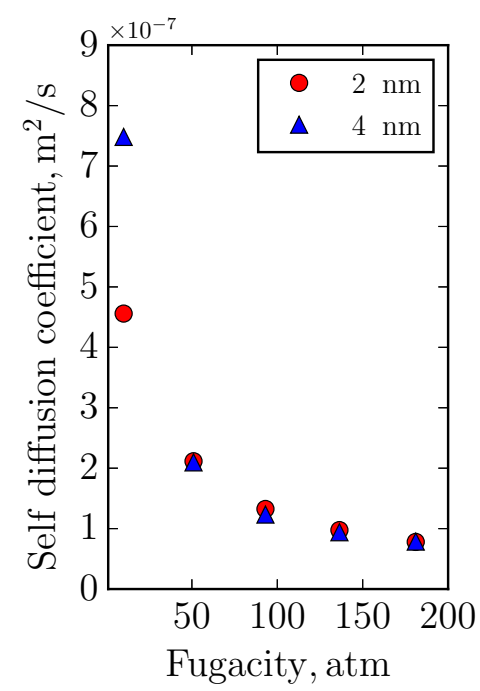

(c) Neon

Figure 3.45: Self-diffusion coefficient of methane and argon in the carbon nanotubes with 2 and 4 nm diameters; (a) Argon (b) Methane

Gas viscosities are calculated using Eq. 3.19. Viscosity values for argon and methane increase approximately linearly with fugacity for both capillaries. For methane and argon, gas viscosity values in the $2 \mathrm{~nm}$ capillary are higher than those in $4 \mathrm{~nm}$ capillary. The viscosity values of neon for the two capillaries are almost the same for fugacities less than $90 \mathrm{~atm}$. As pressure increases above $100 \mathrm{~atm}$, the differences between the values in the two capillaries become larger. The neon viscosities are less than argon and methane. 


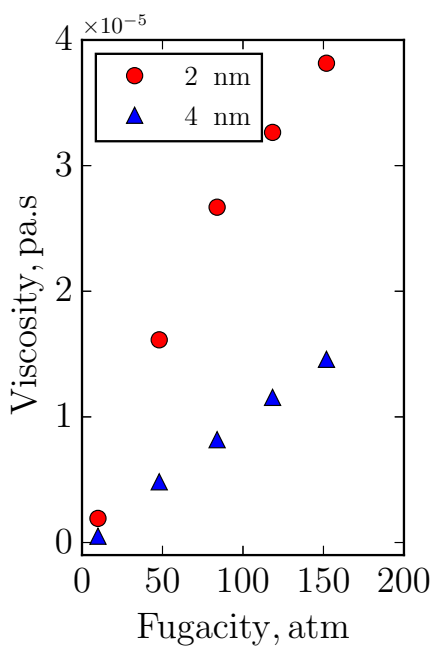

(a) Argon

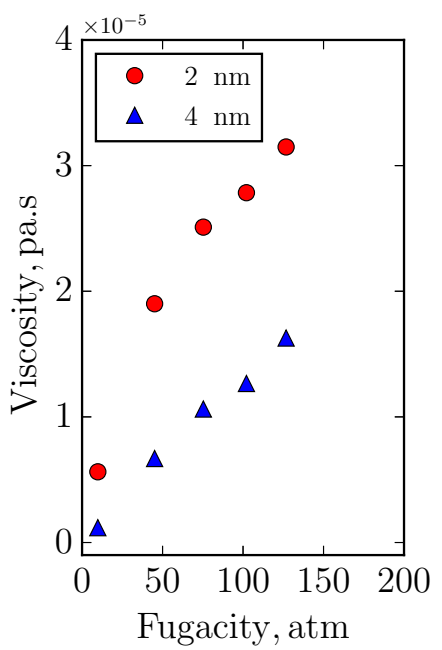

(b) Methane

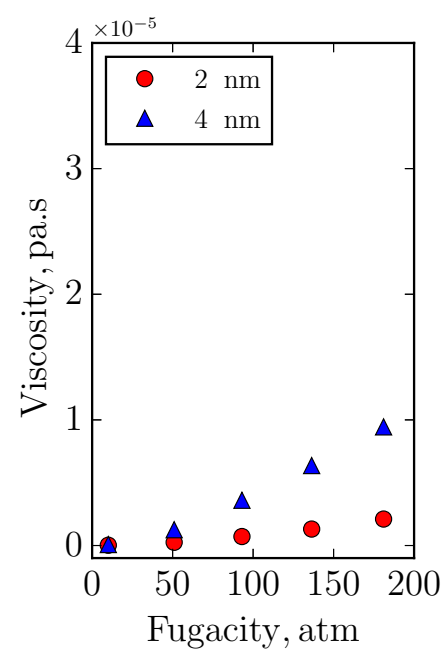

(c) Neon

Figure 3.46: Viscosity of gases in the 2 and $4 \mathrm{~nm}$ capillaries as a function of fugacity; (a) Argon (b) Methane (c) Neon.

In order to compare the calculated gas viscosities with those in Karniadakis et al. [52], the ratio of $\frac{\mu_{\text {actual }}}{\mu_{\text {nominal }}}$ is plotted against the Knudsen number in Fig.3.47. The Knudsen number is calculated using,

$$
K n=\frac{1}{\sqrt{2} n_{v} \pi r d^{2}},
$$

where $n_{v}$ is number density, $r$ is radius of capillary, and $d$ is the molecular diameter. According to Karniadakis et al. [52] model, the actual to nominal viscosity ratio is calculated as,

$$
\frac{\mu_{\text {actual }}}{\mu_{\text {nominal }}}=\frac{1}{1+2.2 K n}
$$

In the $4 \mathrm{~nm}$ capillary, the computed viscosities fall below the nominal viscosities as the ratio is less than one for all gases for the Knudsen numbers tested. On the other hand, the viscosities of the adsorbing gases (argon and methane) in the $2 \mathrm{~nm}$ capillary decreases from above to below its nominal values as Knudsen number increases from 0.5 to 2.5. For argon and methane, the ratio of actual to nominal viscosity is not only a function of Knudsen number, but also the capillary size. Therefore, the Karniadakis et al. [52] model which is developed for systems without adsorption (such as inorganic conduits), cannot be used for organic nanopores. A comparison of molecular simulation results (and employing Hippler et al. [41] model) and Karniadakis et al. [52] model prediction shows that the latter is underestimating adsorptive gas viscosities in the $2 \mathrm{~nm}$ capillary for Knudsen 
numbers less than 2 .

Neon viscosity values in both capillaries are below their nominal values. Predicted viscosity values by the Karniadakis et al. [52] model are close to the ones predicted by the molecular simulations. It should be pointed out that the low Knudsen flow viscosity results for neon in the $2 \mathrm{~nm}$ capillary are not available.

To see the effect of Knudsen number on the computed viscosity values of the argon and methane, the viscosity values are plotted in Fig. 3.48. For each gas in this figure, the viscosity values are different in different capillary sizes. However, for both gases in each capillary, similar viscosity values are observed for each Knudsen number.

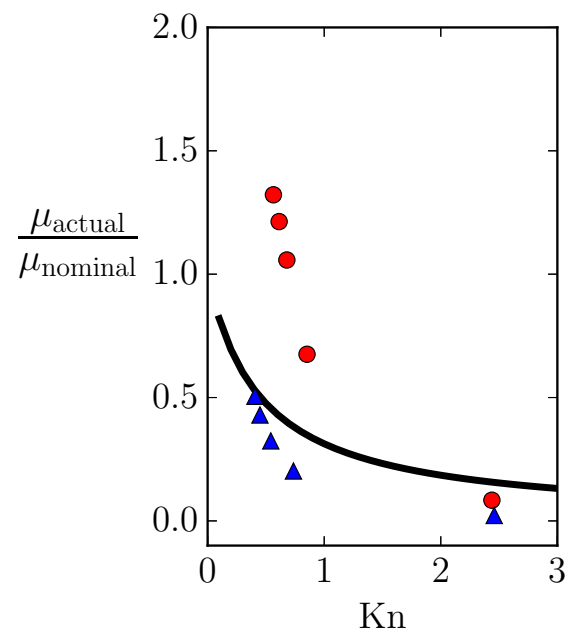

(a) Argon

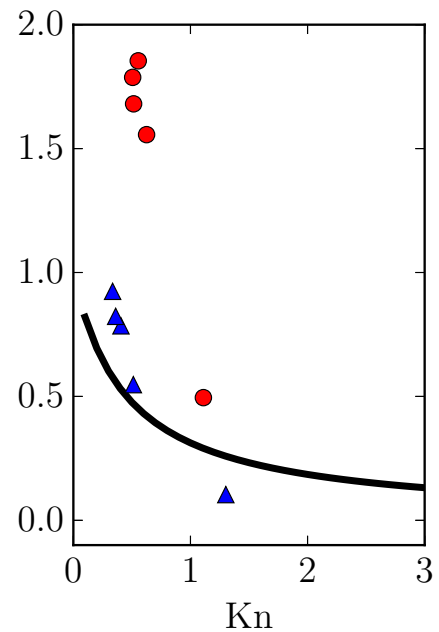

(b) Methane

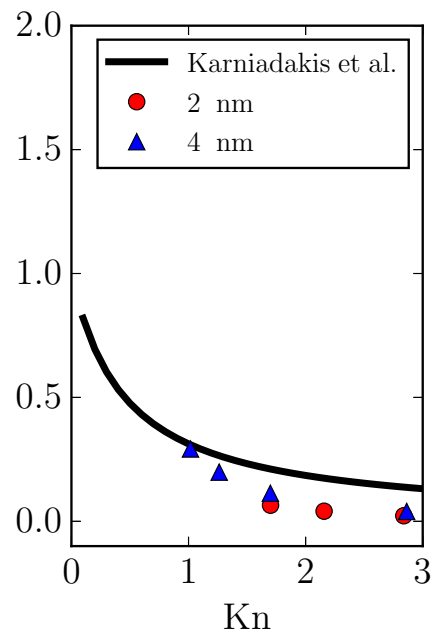

(c) Neon

Figure 3.47: Viscosity of gases in the 2 and $4 \mathrm{~nm}$ capillaries as a function of Knudsen number; (a) Argon (b) Methane (c) Neon. 


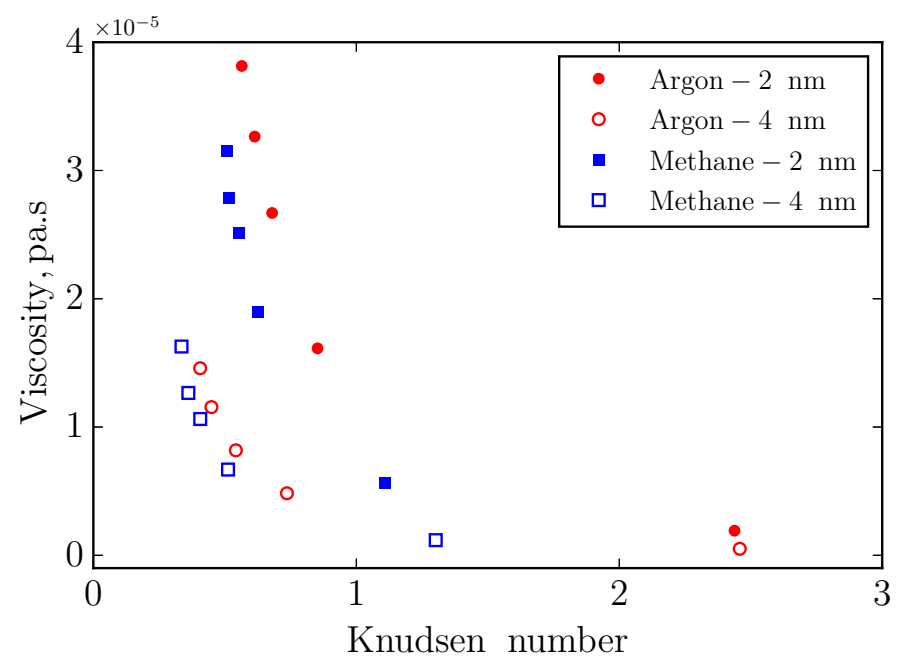

Figure 3.48: Viscosity vs Kn

\subsubsection{Conclusions}

In this work, transport of gases with high adsorbing capacity (methane and argon) are compared with the ones with low adsorbing capacity (neon) in carbon-based capillaries with 2, 4, 6, and 8 $n m$ diameters. Monte Carlo simulation are performed within Grand Canonical ensemble to predict the adsorption properties (excess and absolute) of investigated gases. DCV-GCMD simulations are then carried out at different pressures and pressure gradients. Self, transport, and corrected diffusion coefficients are also calculated. Density, mass flux, and velocity profiles of the gases are compared. Furthermore, contribution of adsorbed phase transport is investigated by the transport of gases with different adsorption capacities. Effect of capillary diameter, average pore pressure, and pressure gradient on the fluid transport in the capillaries are studied.

Based on the simulations results, as the pressure increases, the amount of adsorbed gas molecules increases and tend to saturate at high pressures. The absolute adsorption of a particular gas is higher in $4 \mathrm{~nm}$ capillary diameters compared with those of $2 \mathrm{~nm}$. Plug-shaped velocity profiles are observed for all the gases and all the capillary sizes studied. Based on the computed mass fluxes, it is shown that the Knudsen diffusion model underestimates the molecular flux. This underestimation for flow of low adsorbing gases is less than that of high adsorbing gases.

The thermodynamic factor in smaller capillaries are higher because molecules collide with capillary walls at higher rates compared with one another. Therefore, the differences between the transport 
and corrected transport diffusivity values are smaller for larger capillaries compared with those for smaller ones.

It is found that less adsorptive gases have higher self-diffusion coefficient where neon demonstrate highest self-diffusivity. Viscosity of all gases reduces as pressure increases and saturates at high pressures. The viscosity of gases in nanopores are lower than their nominal values. It was found that the viscosity models for dilute gases under confinement are not good candidates to be used in organic nanopores due to adsorption of gases to pore surface. For adsorbing gases (with similar adsorbing properties) in a capillary, similar viscosity values are observed for each Knudsen number.

The conclusions made in this study are drawn based on the MD simulation results in smooth structure-less geometries. Transport and adsorption of fluids on geometries with rough surfaces should be done to make more conclusive recommendations on fluid flow and storage in realistic systems such as shale reservoirs. 


\section{Chapter 4}

\section{Carbon Dioxide Sequestration in}

\section{Realistic Kerogen Model}

Carbon capture and sequestration (CCS) in depleted shale gas reservoirs provides an opportunity for underground storage of the $\mathrm{CO}_{2} . \mathrm{CO}_{2}$ has higher affinity to the organic matter of shale compared to $\mathrm{CH}_{4}$. Injecting $\mathrm{CO}_{2}$ into the shale reservoirs can cause the $\mathrm{CH}_{4}$ molecules in the adsorbed state to be desorbed and and replaced by the $\mathrm{CO}_{2}$ molecules (enhanced gas recovery-EGR). Current understanding of the competitive adsorption/desorption process of $\mathrm{CO}_{2}$ and $\mathrm{CH}_{4}$ and its impact on the efficiency of CCS is still insufficient. Furthermore, the transport coefficients describing this process are not well defined.

In EGR and CCS processes in shale, the competitive adsorption equilibrium is of significant importance. There have been many approaches to determine the adsorption isotherms of pure $\mathrm{CO}_{2}$ and pure $\mathrm{CH}_{4}$ to estimate the maximum capacity for $\mathrm{CO}_{2}$ storage and $\mathrm{CH}_{4}$ recovery including the volumetric method [40,71, 77], manometric method [62], and gravimetric method [80]. Adsorption of binary $\mathrm{CO}_{2}-\mathrm{CH}_{4}$ mixtures has also been measured using gravimetric-chromatographic method [79]. Shale is composed of organic nanoporous matter called kerogen. Almost half of the total hydrocarbons is assumed to be in stored in kerogen in the adsorbed state $[24,109]$. Thus, it is necessary to study the adsorption phenomena from a microscopic point of view.

Molecular simulations have been proved to be credible alternatives to experimental measurements 
of species adsorption in micoroporous materials [4]. GCMC simulations have been extensively used to study the pure species adsorption in microporous carbon [12], graphite nano-conduits [58], and realistic molecular models of coal [113] and shale [25] and microporous carbon [12].

There are few studies on species diffusion in EGR process in the literature. There are a few mechanisms, such as inhomogeneous fluid distribution of the species, adsorption selectivity, and apparent viscosity that manifest themselves into different diffusion coefficients, namely self-, corrected (or Maxwell-Stefan), and transport diffusion coefficients. The transport diffusion coefficients of pure $\mathrm{CO}_{2}$ and pure $\mathrm{CH}_{4}$ and $\mathrm{CO}_{2}-\mathrm{CH}_{4}$ mixtures can be calculated from their trajectories $[92,113]$.

The objective for this work is to shed light on the the diffusion phenomena of the binary mixtures by performing molecular simulations in carbon-based organic nanochannels. Adsorption of pure $\mathrm{CH}_{4}$ and pure $\mathrm{CO}_{2}, \mathrm{CO}_{2}-\mathrm{CH}_{4}$ mixtures, and also adsorption selectivity values of $\mathrm{CO}_{2}$ over $\mathrm{CH}_{4}$ are computed by performing GCMC simulations. EMD simulations are also performed to calculate the Onsager transport diffusion coefficient matrix and the Fickian diffusion coefficients via thermodynamic factors at varying pressure and species mole fraction.

\subsection{Diffusion of Binary Mixtures}

Three different phenomenological approaches are generally used to model the diffusion, namely self-, corrected (or Maxwell-Stefan), and transport diffusion coefficients. In Fick's formulation, the fluxes, $(\boldsymbol{N})$, are related to the gradients of concentrations, $\nabla \boldsymbol{c}$, with Fick's diffusivity matrix, $D^{T}$, being the proportionality constant,

$$
(\boldsymbol{N})=-\rho\left[D^{T}\right](\nabla \boldsymbol{c})
$$

or in vector notation,

$$
N_{i}=-\rho D_{i j}^{T} \frac{\partial c}{\partial x_{j}}
$$

where $N_{i}$ is the molecular flux of species $i$ in a mixture (molecules $\left./ m^{2} . s\right), \rho$ is the density number $\left(\mathrm{kg} / \mathrm{m}^{3}\right), c_{i}$ is the concentration, and $D^{T}$ is the matrix of Fick's diffusivities $\left(\mathrm{m}^{2} / \mathrm{s}\right)$, which is strongly dependent on mixture composition and loadings. Another popular formulation is Onsager's. In this formulation, the fluxes are casted as a function of gradients of chemical potential,

$$
(\boldsymbol{N})=-[L](\nabla \boldsymbol{\mu})
$$


The Onsager matrix, $[L]$, is a symmetric matrix. For a mixture of species $i$ and $j$, Onsager matrix can be written as,

$$
L_{i j}=\frac{1}{6 N} \lim _{t \rightarrow \infty} \frac{1}{t}\left\langle\left(\sum_{l=1}^{N_{i}}\left(r_{l, i}(t)-r_{l, i}(0)\right) \cdot\left(\sum_{k=1}^{N_{j}}\left(r_{k, j}(t)-r_{k, j}(0)\right)\right\rangle\right.\right.
$$

where $r_{l, i}(t)$ is the position of $l^{t h}$ molecule of component $i$ at time $t$. The chemical potential gradient can be related to concentration gradient as,

$$
\begin{gathered}
\frac{c_{i}}{k_{B} T} \nabla \mu_{i}=\sum_{j} \Gamma_{i j} \nabla c_{j}, \quad \Gamma_{i j}=\frac{\theta_{i}}{\theta_{j}} \frac{\partial \ln f_{i}}{\partial \ln \theta_{j}} \\
\theta_{i}=\frac{c_{i}}{c_{i, s a t}}
\end{gathered}
$$

where $\theta_{i}$ is the fractional occupancy of component $i, c_{i, s a t}$ is saturated molar concentration of adsorbed species, $f_{i}$ is the fugacity of component $i$, and $\Gamma_{i j}$ is the matrix of thermodynamic factors. If the binary mixture adsorption isotherms can be modeled using multicomponent Langmuir isotherm, then the elements of matrix of thermodynamic factors can be written as,

$$
\Gamma_{i j}=\delta_{i j}+\frac{\theta_{i}}{1-\theta_{1}-\theta_{2}}
$$

Therefore, the matrix of Onsager diffusion coefficients can be converted to those of Fickian as,

$$
\left[D^{T}\right]=[L]\left[\begin{array}{cc}
1 / \theta_{1} & 0 \\
0 & 1 / \theta_{2}
\end{array}\right][\Gamma]
$$

While Fick's and Onsager formulations are phenomenological approaches, the Maxwell-Stefan formulation balances diffusive and drag forces. Diffusion of species $i$ is described as,

$$
\rho \frac{\theta_{i}}{k_{B} T} \nabla \mu_{i}=\sum_{\substack{j=1 \\ i \neq j}}^{n} \frac{c_{j} N_{i}-N_{i} c_{j}}{c_{i, s a t} c_{j, s a t} D_{i j}^{M S}}+\frac{N_{i}}{c_{i, s a t} D_{i}^{M S}}
$$

where $D_{i}^{M S}$ is the Maxwell-Stefan diffusivity coefficient of component $i$ and $D_{i j}^{M S}$ are the binary exchange Maxwell-Stefan diffusivities. For single component systems, $D_{i}^{M S}$ is called the corrected diffusivity. It is important to emphasize that all aforementioned formulations of are strictly equivalent. 


\subsection{Binary Adsorption Isotherms}

Grand canonical Monte Carlo simulations of methane and carbon dioxide are performed to investigate the binary adsorption behavior of these two gases in kerogens with different maturities. Simulations are performed at various pressures. The methane and carbon dioxide pressures are changed from 20 to $170 \mathrm{~atm}$ resulting in 36 simulations for each kerogen type. All the simulations are performed at $350 \mathrm{~K}$. The methane and carbon dioxide molecules are modeled using OPLS-AA and COMPASS class2 force fields, respectively.

The adsorption isotherms of all kerogen types are plotted in Figs. 4.1 and 4.2. The adsorbed amount of $\mathrm{CO}_{2}$ is higher than $\mathrm{CH}_{4}$ for all kerogen types at all pressures due to strong permanent quadrapole moment of $\mathrm{CO}_{2}$. As the pressure of each gas at the pore space increases, its adsorbed amount increases as well and at the same time, the adsorbed amount of other gas reduces. 


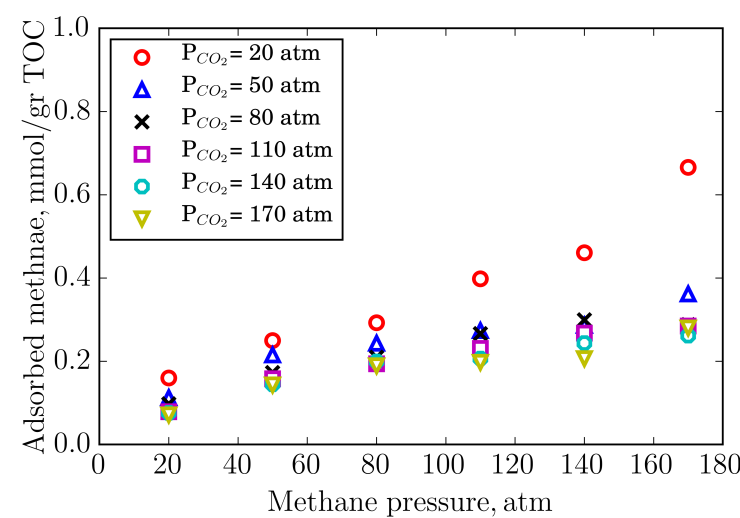

(a) IIA- $\mathrm{CH}_{4}$

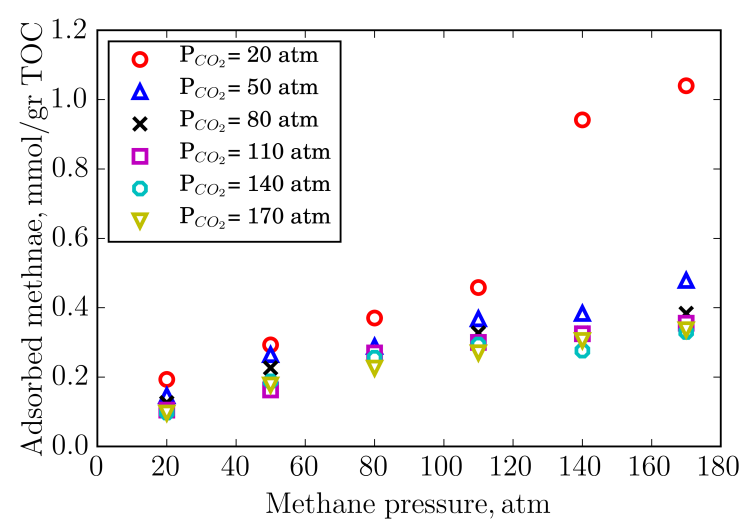

(c) $\mathrm{IIB}-\mathrm{CH}_{4}$

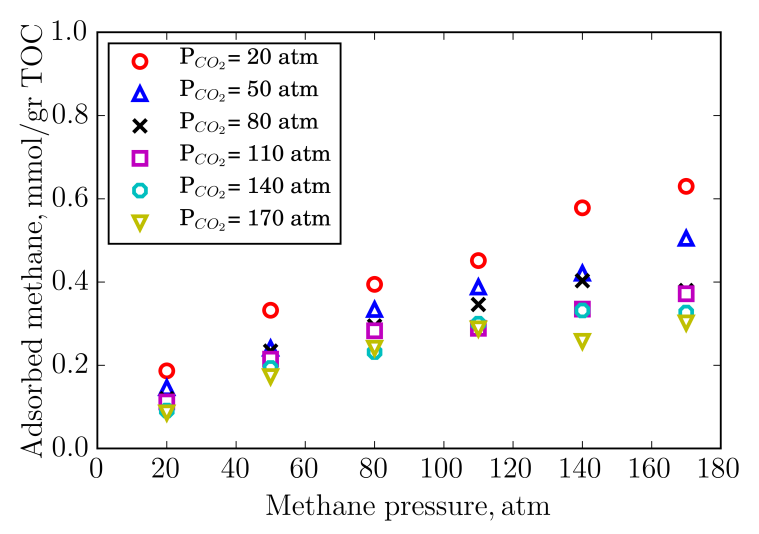

(e) $\mathrm{IIC}-\mathrm{CH}_{4}$

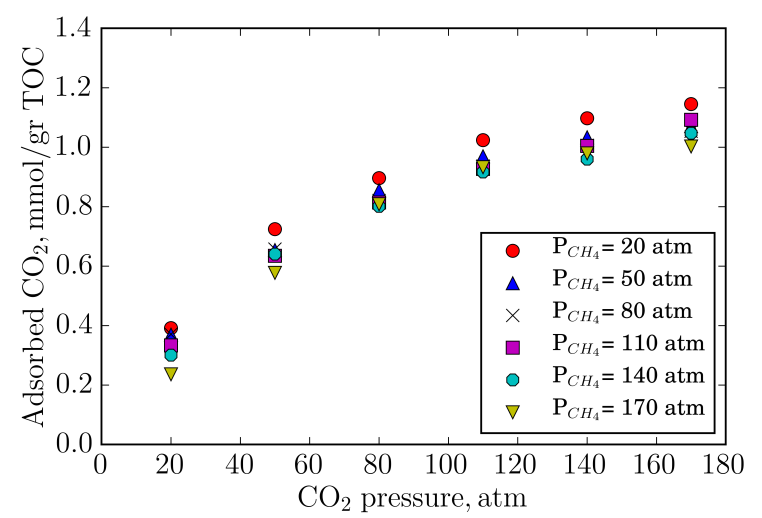

(b) IIA-CO $\mathrm{CO}_{2}$

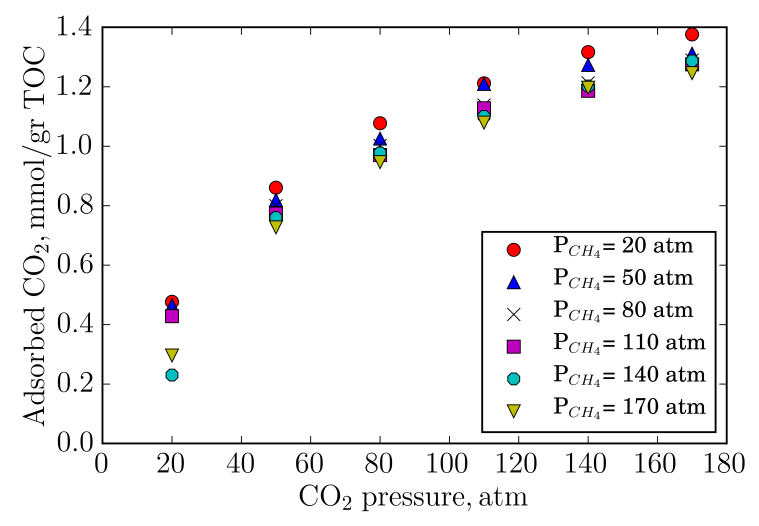

(d) IIB- $\mathrm{CO}_{2}$

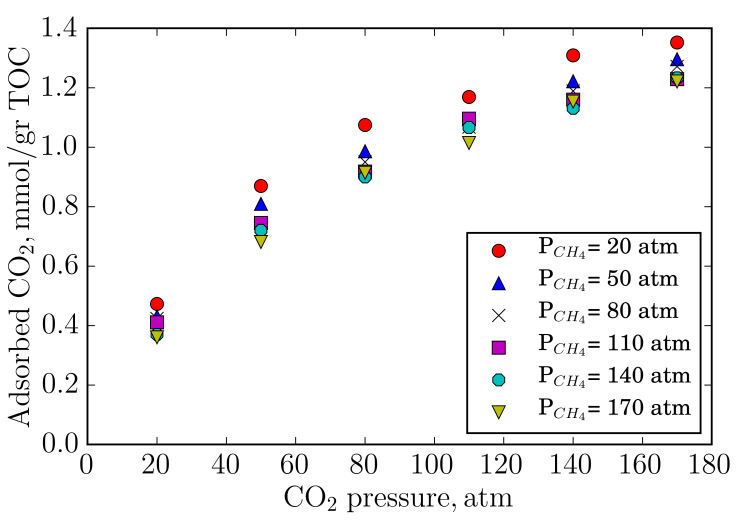

(f) $\mathrm{IIC}-\mathrm{CO}_{2}$

Figure 4.1: Binary adsorption isotherms of $\mathrm{CH}_{4}$ and $\mathrm{CO}_{2}$ for kerogen types IIA, IIB, and IIC. 


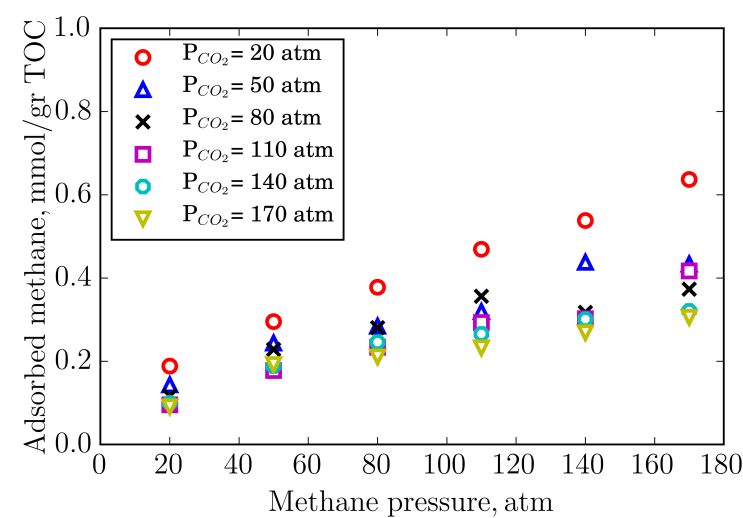

(a) $\mathrm{IID}_{-} \mathrm{CH}_{4}$

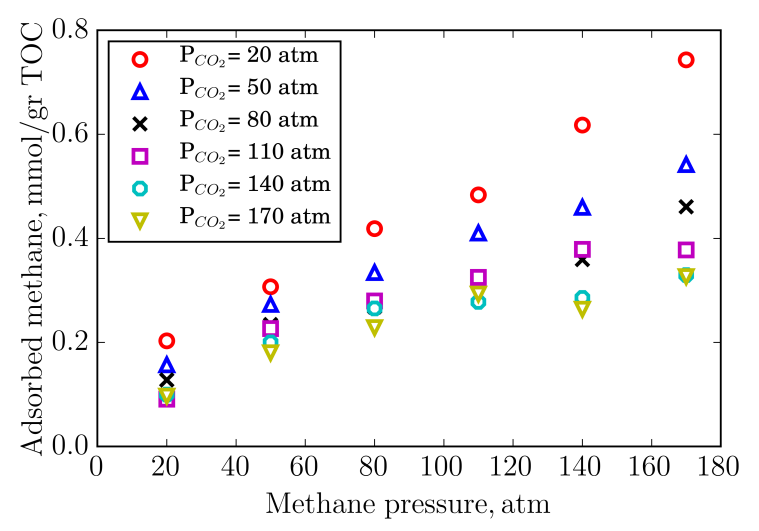

(c) IIIA- $\mathrm{CH}_{4}$

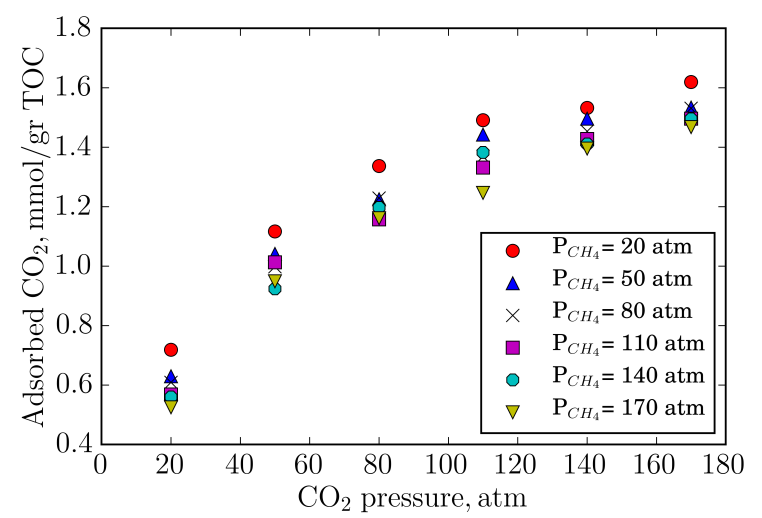

(b) $\mathrm{IID}-\mathrm{CO}_{2}$

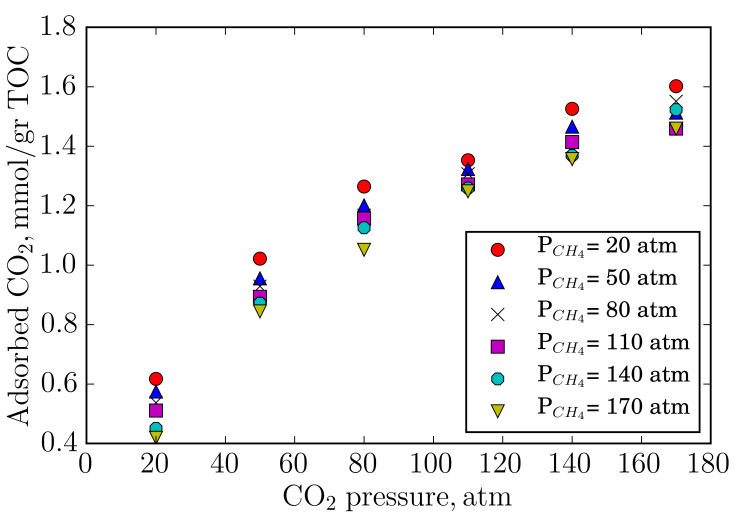

(d) IIIA- $\mathrm{CO}_{2}$

Figure 4.2: Binary adsorption isotherms of $\mathrm{CH}_{4}$ and $\mathrm{CO}_{2}$ for kerogen types IID and IIIA.

For example, for kerogen type IIA (Fig. 4.1a), the amount of adsorbed methane increases from 0.16 to $0.66 \mathrm{mmol} / \mathrm{gr}$ when its pressure increases from 20 to $170 \mathrm{~atm}$ and $\mathrm{CO}_{2}$ pressure is $20 \mathrm{~atm}$ (red hollow circles). When the $\mathrm{CO}_{2}$ pressure increases to $170 \mathrm{~atm}$, the amount of adsorbed methane increases from 0.07 to $0.28 \mathrm{mmol} / \mathrm{gr}$ which demonstrates a reduction by $57 \%$. Looking into Fig. 4.1b, the amount of adsorbed $\mathrm{CO}_{2}$ increases from 0.39 to $1.14 \mathrm{mmol} / \mathrm{gr}$ when the $\mathrm{CO}_{2}$ pressure increases from 20 to $170 \mathrm{~atm}$ at a methane pressure of $20 \mathrm{~atm}$. At a $\mathrm{CH}_{4}$ pressure of $170 \mathrm{~atm}$, the amount of adsorbed $\mathrm{CO}_{2}$ increases from 0.23 to $1 \mathrm{mmol} / \mathrm{gr}$. The amount of $\mathrm{CO}_{2}$ decreased by $41 \%$ and $14 \%$ when the methane pressures are 20 and 170 atm, respectively. This trend can be observed for all kerogen types. The most significant reduction of in methane adsorption amount occurs when $\mathrm{CO}_{2}$ pressure increases from 20 to $50 \mathrm{~atm}$. The adsorption capability of methane does not change significantly for $\mathrm{CO}_{2}$ pressure above 80 atm.

The adsorption isotherms are fitted to Langmuir multicomponent adsorption isotherms as is shown 
in Equation. 4.9. Here and throughout the remainder of this chapter, the subscripts 1 and 2 for concentrations, partial pressures, etc. refer to $\mathrm{CH}_{4}$ and $\mathrm{CO}_{2}$, respectively.

$$
\begin{aligned}
& \frac{c_{1}}{c_{1, \text { sat }}}=\frac{A_{1} P_{1}}{1+A_{1} P_{1}+A_{2} P_{2}} \\
& \frac{c_{2}}{c_{2, \text { sat }}}=\frac{B_{1} P_{2}}{1+B_{1} P_{1}+B_{2} P_{2}}
\end{aligned}
$$

Coefficients obtained for the best fit are listed in Table 4.1.

Table 4.1: Calculated parameters for the binary adsorption isotherms (Equation. 4.9 )

\begin{tabular}{c|c|c|c|c|c}
\hline Parameter & Value-IIA & Value-IIB & Value-IIC & Value-IID & Value-IIIA \\
\hline \hline$A_{1}$ & 0.01538 & 0.00775 & 0.02488 & 0.02174 & 0.01617 \\
\hline$A_{2}$ & 0.02884 & 0.02721 & 0.02607 & 0.02491 & 0.02492 \\
\hline$c_{1, \text { sat }}$ & 0.79194 & 1.59680 & 0.75161 & 0.75345 & 0.97402 \\
\hline$B_{1}$ & 0.01904 & 0.01865 & 0.01961 & 0.02931 & 0.02111 \\
\hline$B_{2}$ & 0.00271 & 0.00281 & 0.00318 & 0.003582 & 0.00294 \\
\hline$c_{2, \text { sat }}$ & 1.49632 & 1.82895 & 1.75217 & 1.92903 & 2.03316 \\
\hline
\end{tabular}

The fitted values are compared with the computed ones in Fig. 4.3. The straight line shows that the fitted values are close to the simulation results. The $\mathrm{CO}_{2}$ fitted data are fitted to Langmuir multicomponent adsorption isotherm with R-squares more than $98 \%$ for all kerogen types. However, methane data are not fitted as good as $\mathrm{CO}_{2}$ data with $\mathrm{R}$-square values more than $85 \%$.

The total adsorption amount can be determined by adding the concentration of each component in Equation 4.9 .

$$
c_{t}=c_{1}+c_{2}
$$

Furthermore, the component mole fractions could be determined as,

$$
y_{1}=\frac{P_{1}}{P_{t}}, \quad y_{2}=1-y_{1}
$$

where $P_{t}$ is the total mixture pressure. The mole fraction of each component in adsorbed phase can be calculated as,

$$
x_{1}=\frac{c_{1}}{c_{t}}, \quad x_{2}=1-x_{1}
$$

Therefore, the equilibrium relationship between free gas phase and adsorbed phase can be expressed 


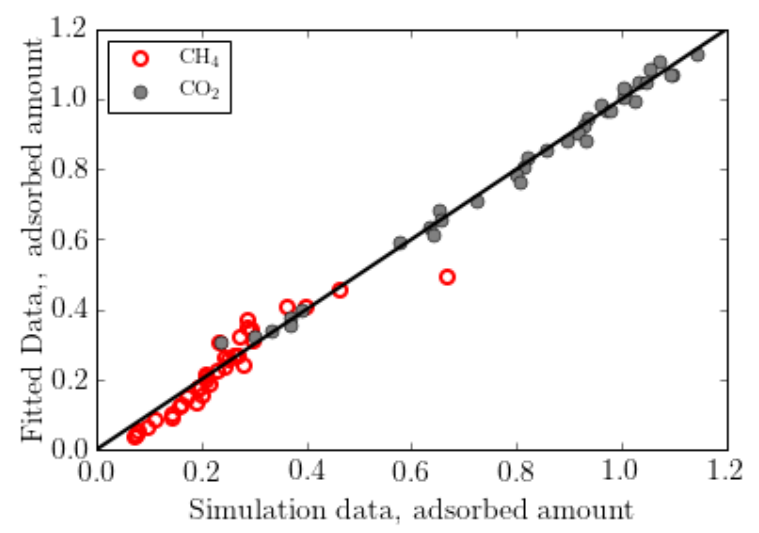

(a) IIA

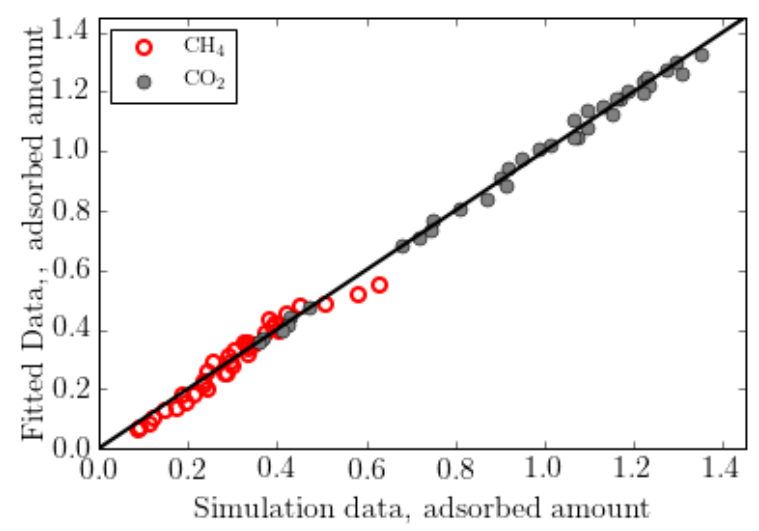

(c) IIC

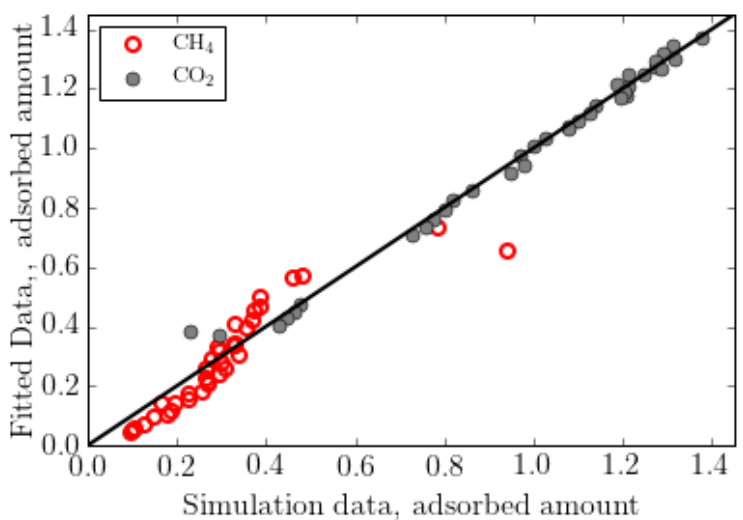

(b) IIB

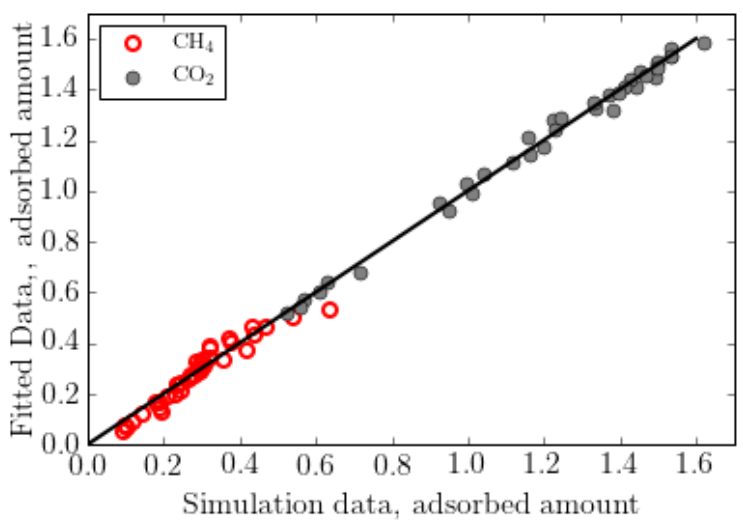

(d) IID

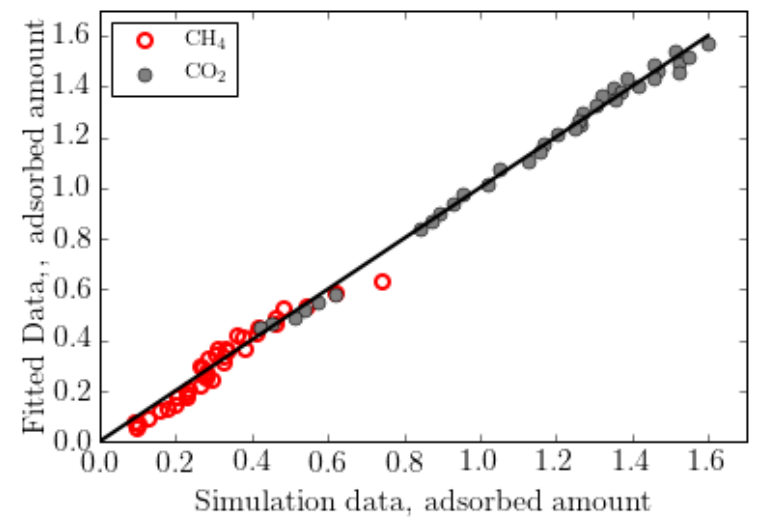

(e) IIIA

Figure 4.3: Comparison between the simulation results and fits using Eqn. 4.9 for the binary mixture adsorption for $\mathrm{CH}_{4}$ (red open circles) and $\mathrm{CO}_{2}$ (filled circles). The line denotes exact agreement. 
as,

$$
\frac{x_{1}}{x_{2}}=\frac{c_{1, \text { sat }}}{c_{2, \text { sat }}} \frac{A_{1} y_{1}}{A_{2} y_{2}} \frac{1+B_{1} P_{1}+B_{2} P_{2}}{1+A_{1} P_{1}+A_{2} P_{2}}
$$

The separation of methane (or carbon dioxide) between free gas and adsorbed phases is called selectivity ratio and is defined as,

$$
\alpha_{1}=\frac{x_{1} / y_{1}}{x_{2} / y_{2}}=\frac{c_{1, s a t}}{c_{2, s a t}} \frac{A_{1}}{A_{2}} \frac{1+B_{1} P_{1}+B_{2} P_{2}}{1+A_{1} P_{1}+A_{2} P_{2}}
$$

where $\alpha_{1}$ is the selectivity of methane over carbon dioxide. The selectivity is depends on temperature, pressure, and composition of the gas mixture and type of adsorbent. Selectivity values near one indicate that the mixture is not separable by an adsorption process.

Figs. 4.4, 4.6, 4.7, 4.8, and 4.9 demonstrate the $\mathrm{x}-\mathrm{y}$ diagrams and selectivity values for different kerogen types at various total pressures $\left(P_{t}\right)$. The red squares demonstrate the $\mathrm{x}_{C H_{4}}-\mathrm{y}_{C H_{4}}$ plots and green circles indicate the $\mathrm{x}_{\mathrm{CO}_{2}}-\mathrm{y}_{\mathrm{CO}_{2}}$ diagrams. The selectivity values of carbon dioxide over methane are determined based on x-y values of each component using Equation 4.14. For all kerogen types, selectivity values are above one for all total pressures and gas compositions demonstrating that carbon dioxide have higher adsorption capabilities than methane. The selectivity values against carbon dioxide composition $\left(\mathrm{y}_{\mathrm{CO}_{2}}\right)$ indicate a maximum value at a $\mathrm{CO}_{2}$ composition less than 0.5 depending on kerogen type and total pressure. At some of selectivity graphs, the maximum point is not observable due to limited number of points. After the maximum point, the selectivity reduces as carbon dioxide bulk composition increases. Comparing different kerogen types, the selectivity values are within the same range of 2 to 7 . At the same composition, as the pressure increases, the selectivity of carbon dioxide over methane increases for pressure range tested. For example, at 50-50\% composition of $\mathrm{CO}_{2}-\mathrm{CH}_{4}$, the selectivity value increases from 2.45 to 3.94 as total pressure increases from 40 to 280 atm for kerogen type IIA (Fig. 4.5). As the kerogen becomes more mature, the selectivity values are relatively increasing. 

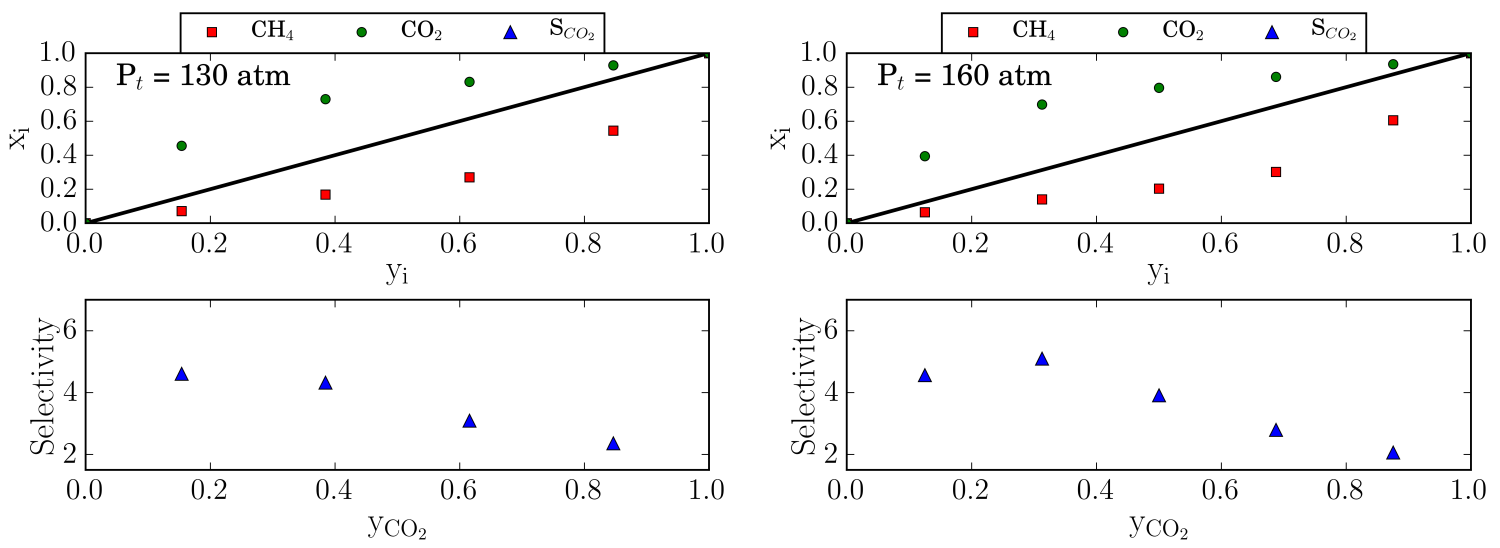

(a) $\mathrm{P}_{t}=130 \mathrm{~atm}$

(b) $\mathrm{P}_{t}=160 \mathrm{~atm}$
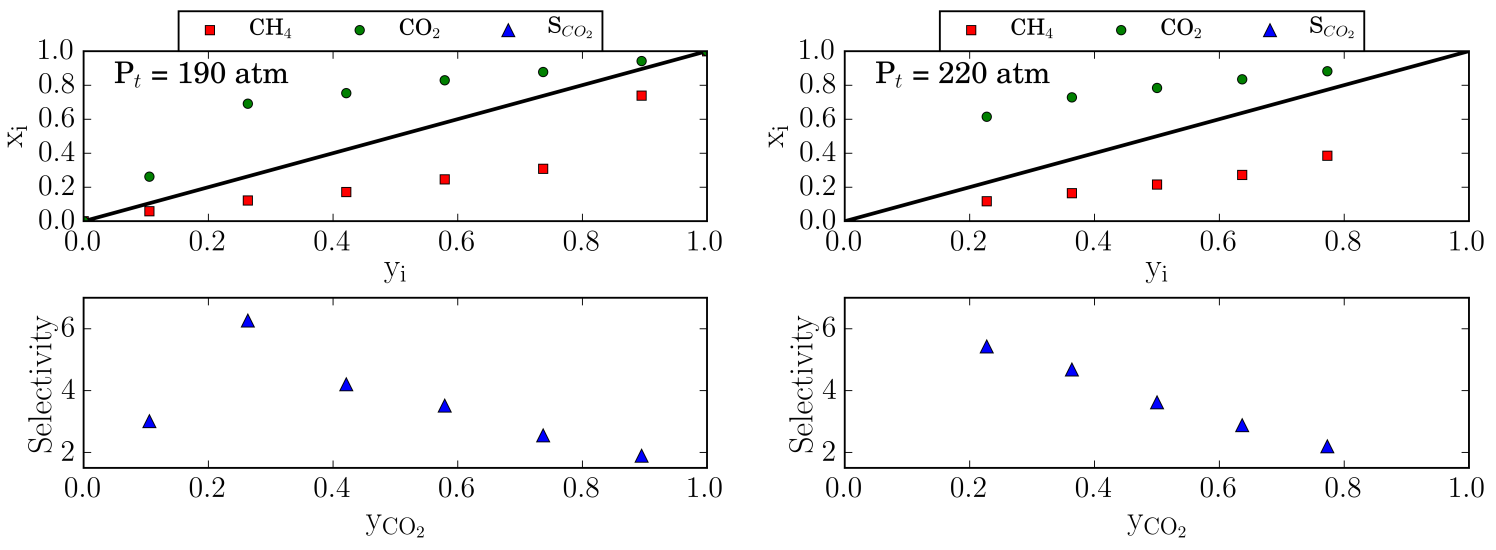

(c) $\mathrm{P}_{t}=190 \mathrm{~atm}$

(d) $\mathrm{P}_{t}=220 \mathrm{~atm}$

Figure 4.4: Selectivities and x-y diagrams at different total pressures for kerogen type IIA.

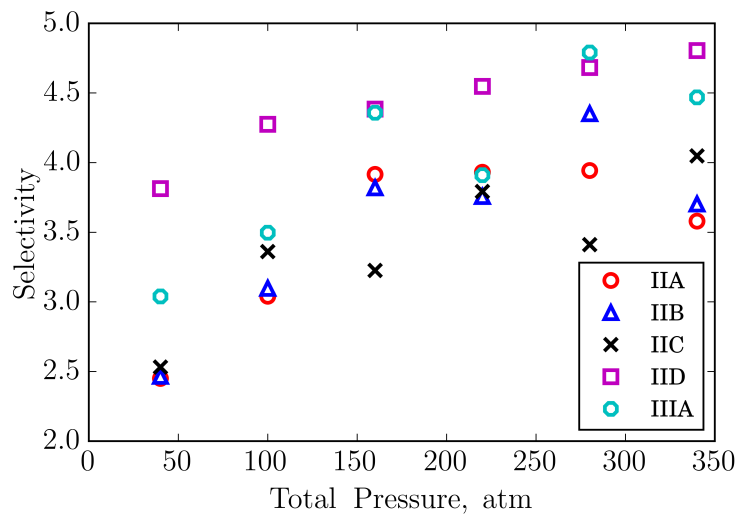

Figure 4.5: Selectivity values of different kerogen types at 50-50\% gases composition. 

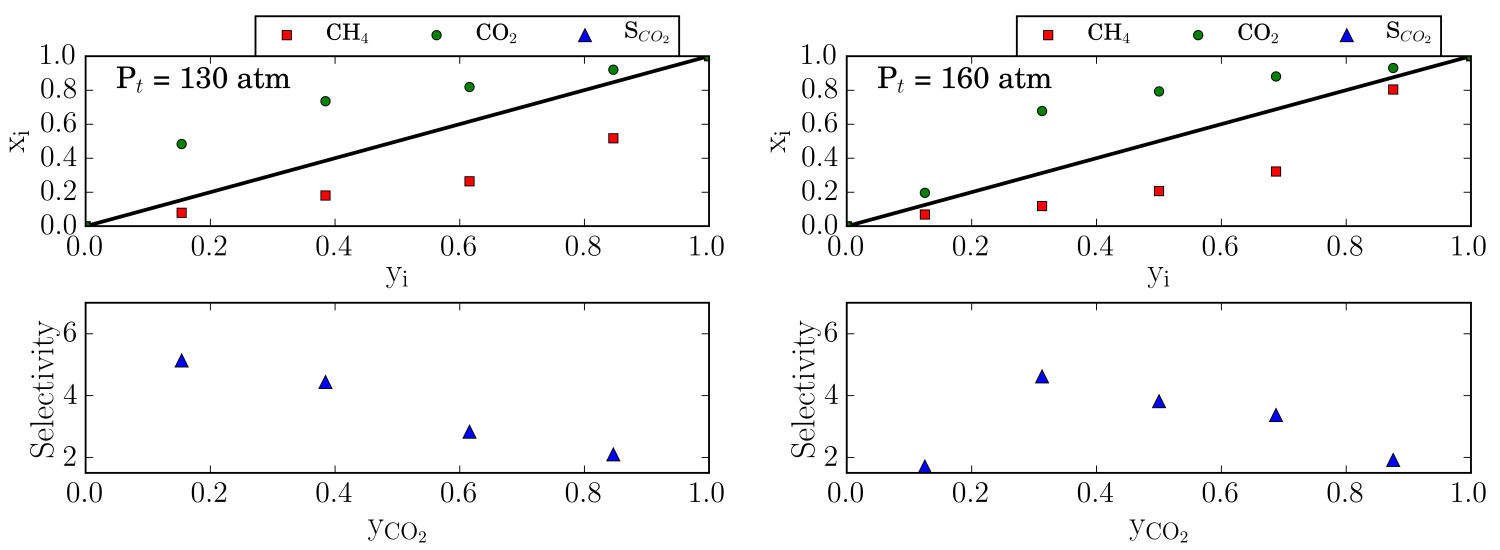

(a) $\mathrm{P}_{t}=130 \mathrm{~atm}$

(b) $\mathrm{P}_{t}=160 \mathrm{~atm}$
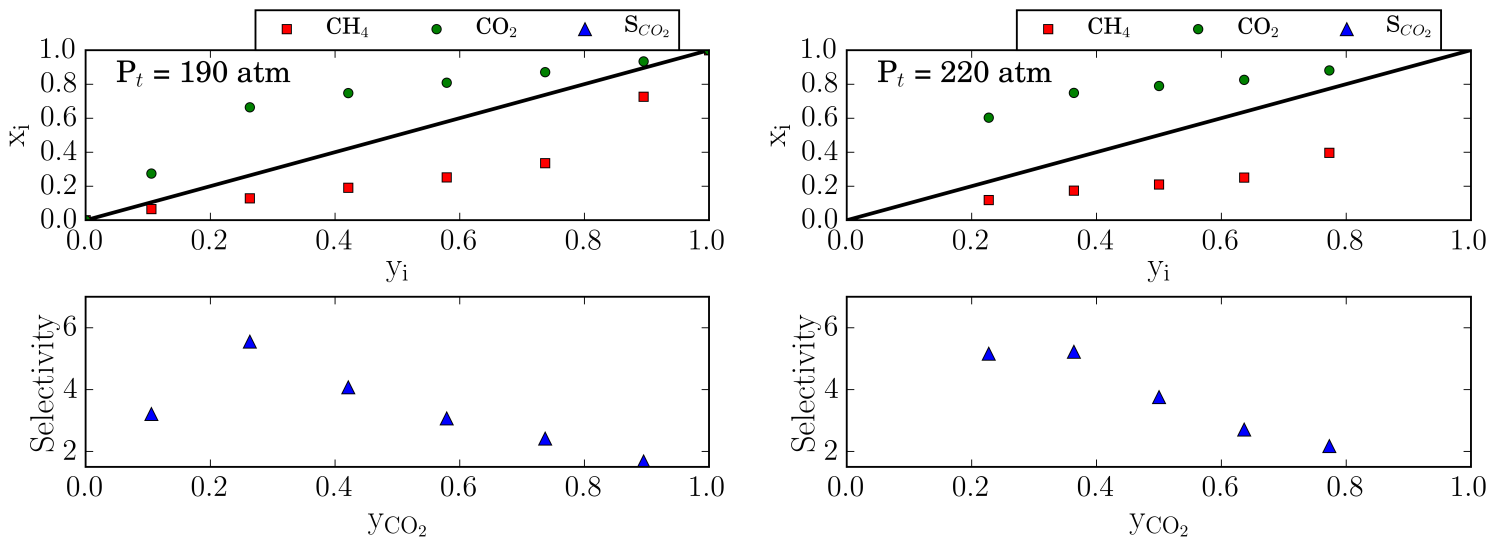

(c) $\mathrm{P}_{t}=190 \mathrm{~atm}$

(d) $\mathrm{P}_{t}=220 \mathrm{~atm}$

Figure 4.6: Selectivities and $\mathrm{x}-\mathrm{y}$ diagrams at different total pressures for kerogen type IIB. 

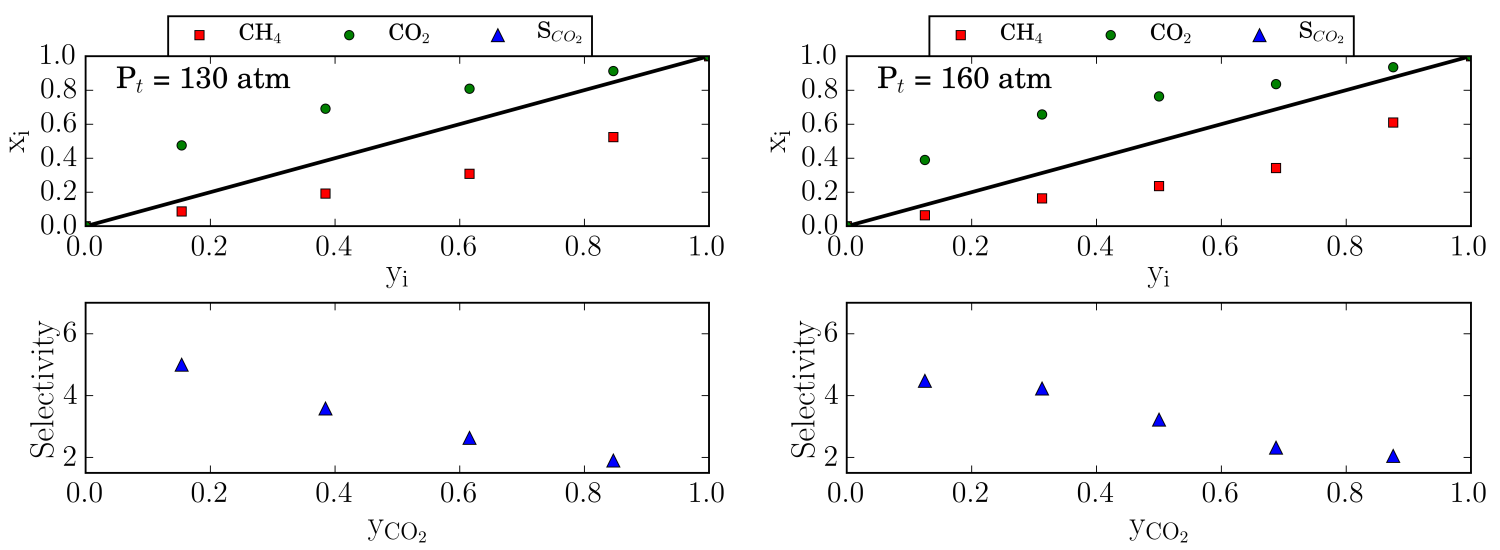

(a) $\mathrm{P}_{t}=130 \mathrm{~atm}$

(b) $\mathrm{P}_{t}=160 \mathrm{~atm}$
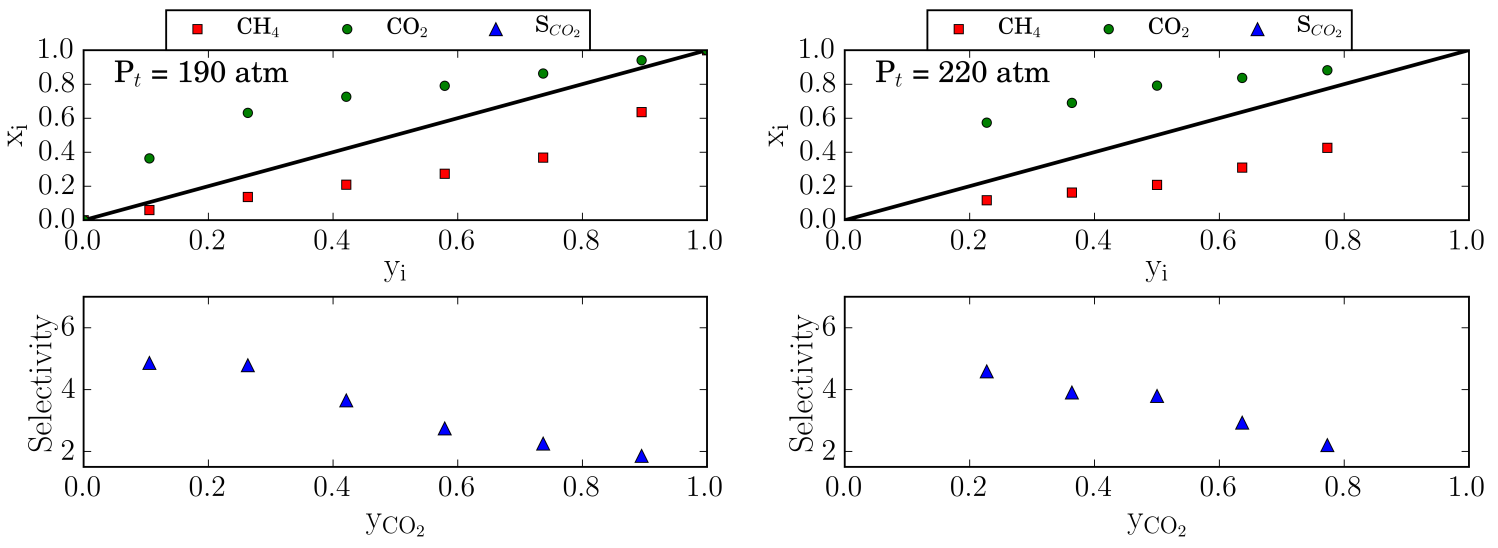

(c) $\mathrm{P}_{t}=190 \mathrm{~atm}$

(d) $\mathrm{P}_{t}=220 \mathrm{~atm}$

Figure 4.7: Selectivities and $\mathrm{x}-\mathrm{y}$ diagrams at different total pressures for kerogen type IIC. 

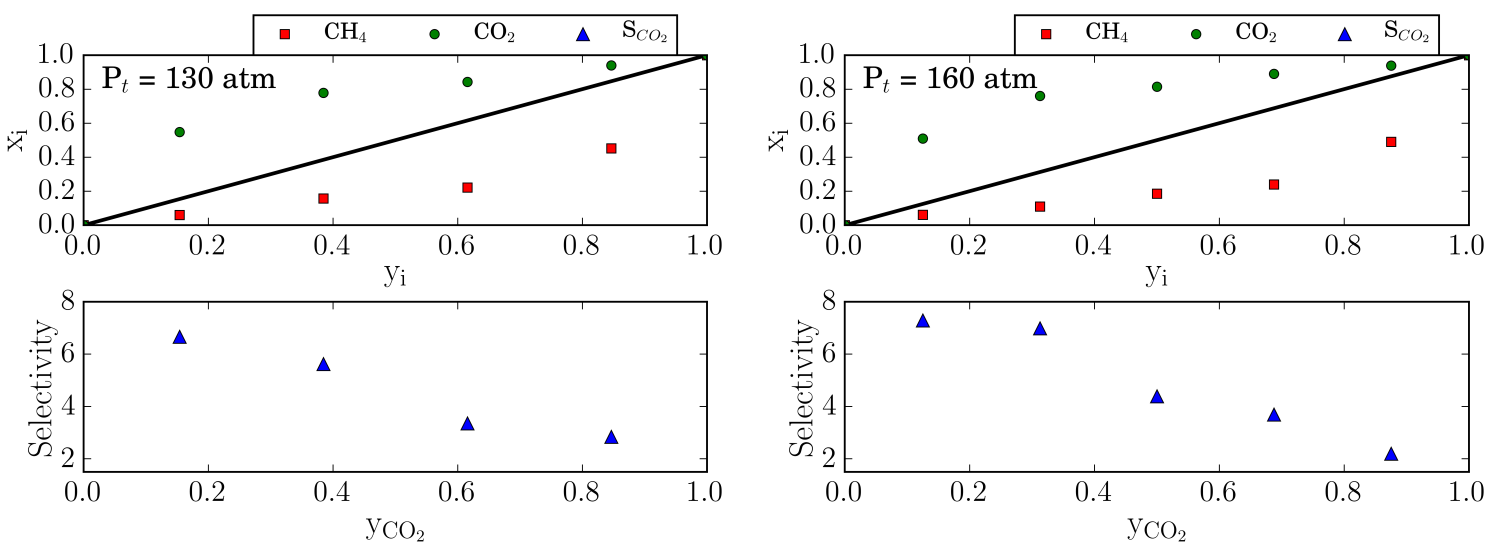

(a) $\mathrm{P}_{t}=130 \mathrm{~atm}$

(b) $\mathrm{P}_{t}=160 \mathrm{~atm}$
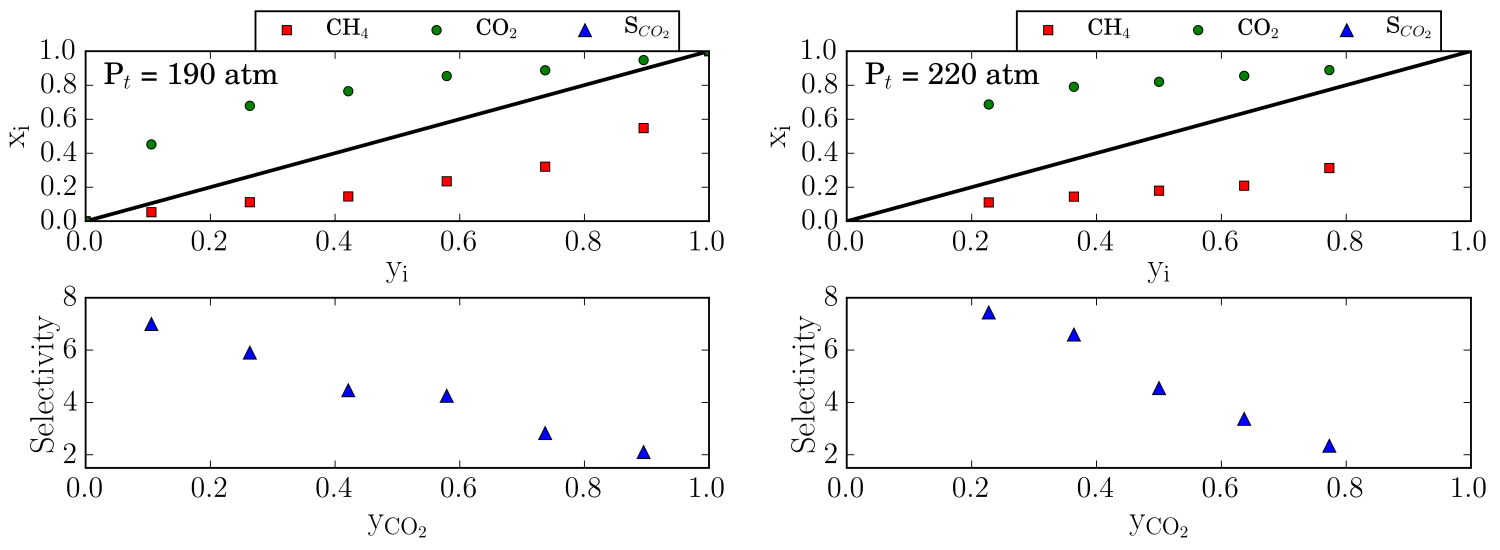

(c) $\mathrm{P}_{t}=190 \mathrm{~atm}$

(d) $\mathrm{P}_{t}=220 \mathrm{~atm}$

Figure 4.8: Selectivities and $\mathrm{x}-\mathrm{y}$ diagrams at different total pressures for kerogen type IID. 

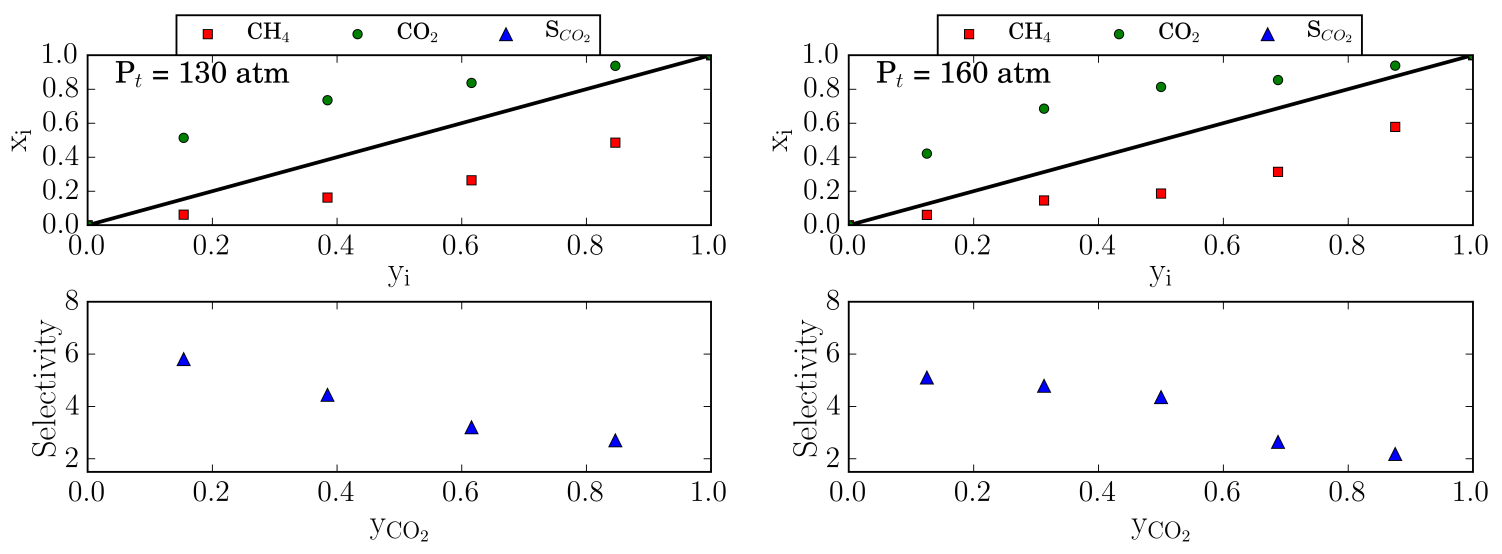

(a) $\mathrm{P}_{t}=130 \mathrm{~atm}$

(b) $\mathrm{P}_{t}=160 \mathrm{~atm}$
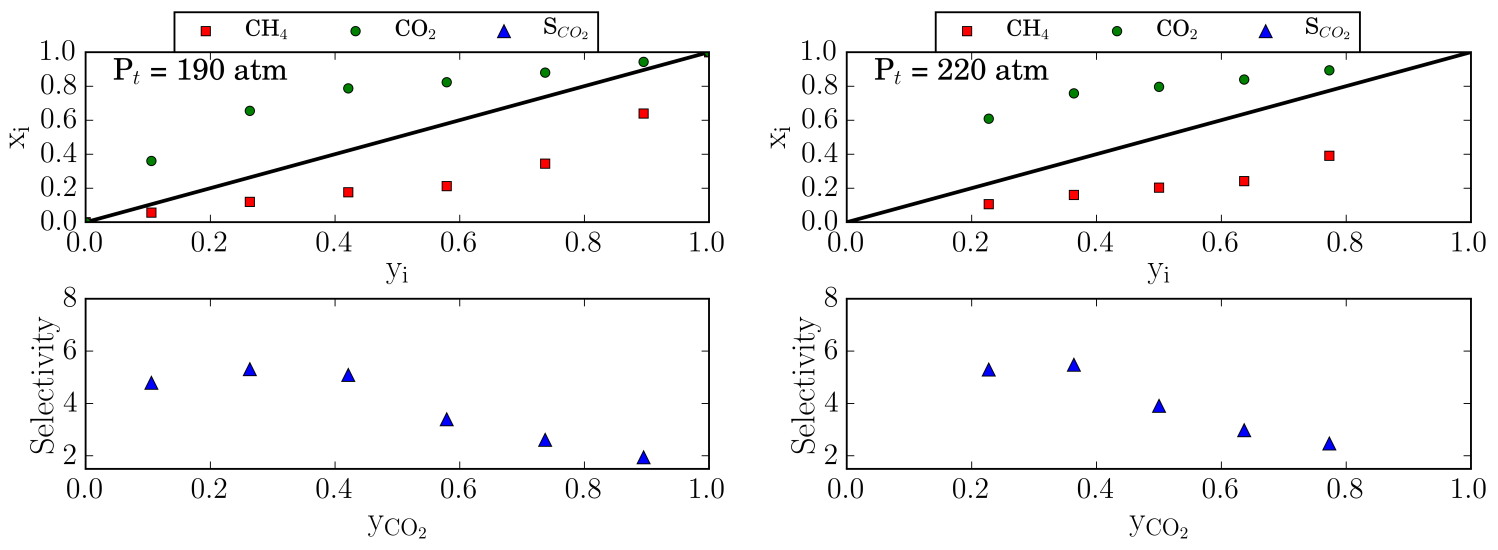

(c) $\mathrm{P}_{t}=190 \mathrm{~atm}$

(d) $\mathrm{P}_{t}=220 \mathrm{~atm}$

Figure 4.9: Selectivities and x-y diagrams at different total pressures for kerogen type IIIA.

\subsection{Binary Diffusion Coefficients}

Diffusion of gases is affected by presence of other components. When $\mathrm{CO}_{2}$ is injected into organic nanopores, concentration $\mathrm{CO}_{2}$ will increase in nanopores and competitive adsorption of methane and carbon dioxide affect the diffusion coefficients of methane. From practical point of view, a reliable procedure for estimating Onsager diffusion coefficients are needed to be used for simulation of $\mathrm{CO}_{2}$ sequestration processes.

Equilibrium molecular dynamics simulations (EMD) are performed to calculate the diffusion coefficients based on trajectory of methane and carbon dioxide molecules for a wide range of pressure. Equation 4.4 is used to determine the Onsager diffusion coefficients. 
Diffusion coefficients are plotted in heat maps plotted in Figs. 4.10, 4.11, 4.12, 4.13, and 4.14. As methane pressure increases while $\mathrm{CO}_{2}$ pressure is kept constant, its Onsager diffusion coefficients increases. Since the carbon dioxide have higher adsorption capabilities than methane, its Onsager diffusion coefficients are smaller than those of methane. Similar trends are observed for kerogen types IIA, IIB, IIC, IID, and IIIA. The off-diagonal Onsager diffusion coefficients (Figs. 4.10b and 4.11b) are almost one order of magnitude less than the diagonal terms.

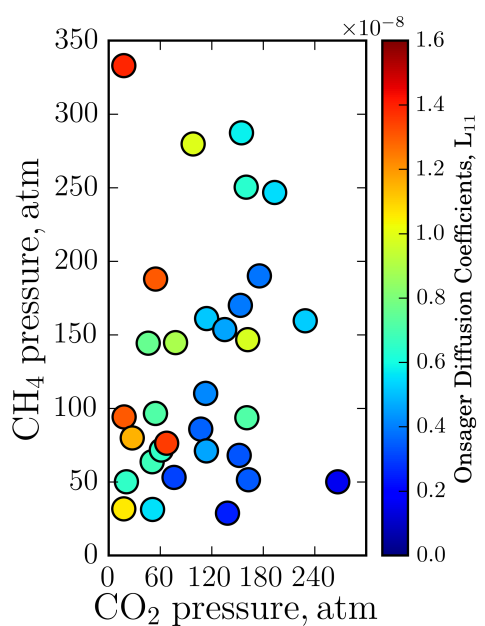

(a) $\mathrm{CH}_{4}-\mathrm{CH}_{4}$

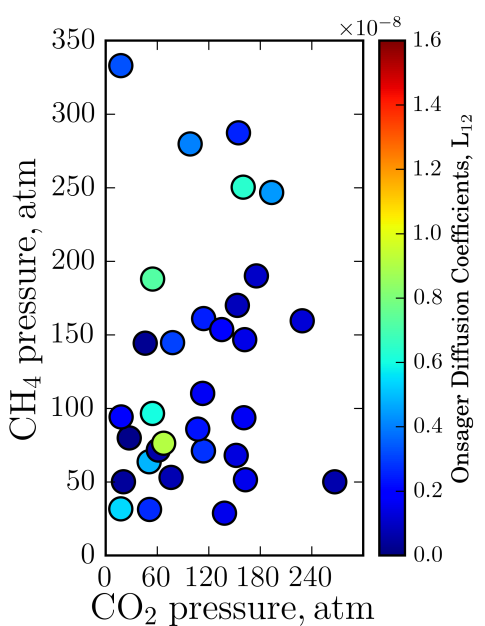

(b) $\mathrm{CH}_{4}-\mathrm{CO}_{2}$

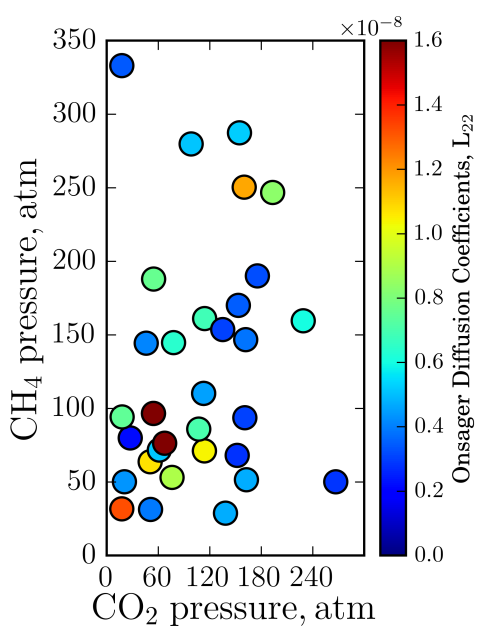

(c) $\mathrm{CO}_{2}-\mathrm{CO}_{2}$

Figure 4.10: Onsager diffusion coefficients of $\mathrm{CH}_{4}-\mathrm{CO}_{2}$ mixtures in the channel; (a) $L_{11}$ (b) $L_{12}$ (c) $L_{22}$.

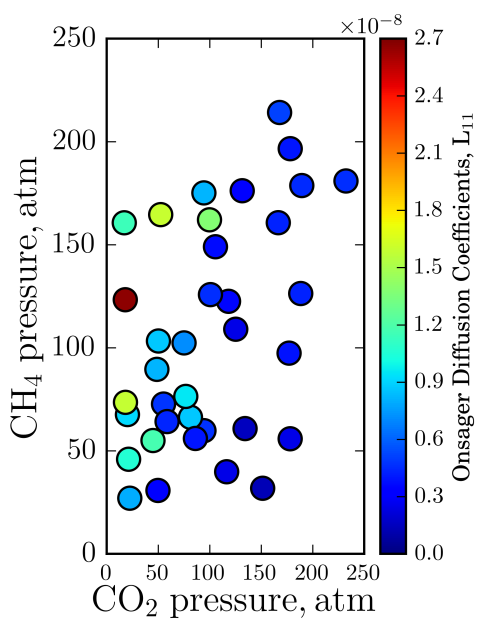

(a) $\mathrm{CH}_{4}-\mathrm{CH}_{4}$

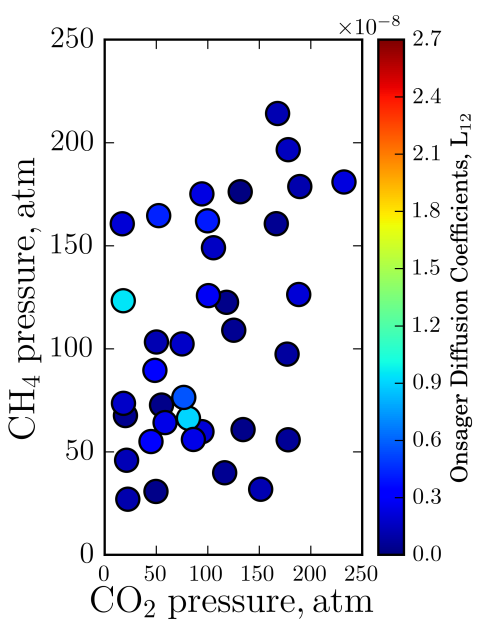

(b) $\mathrm{CH}_{4}-\mathrm{CO}_{2}$

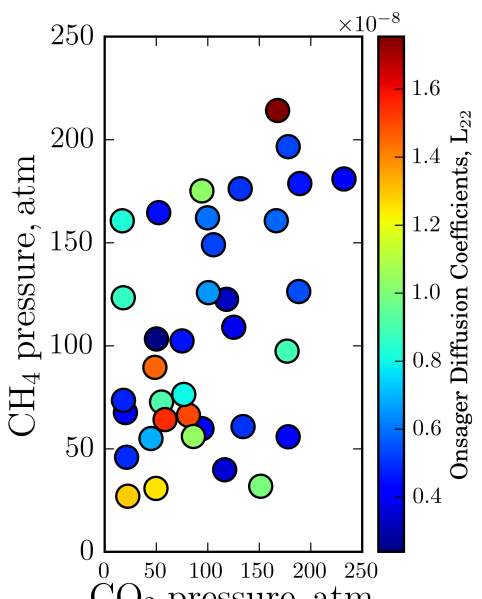

(c) $\mathrm{CO}_{2}-\mathrm{CO}_{2}$

Figure 4.11: Onsager diffusion coefficients of $\mathrm{CH}_{4}-\mathrm{CO}_{2}$ mixtures in the channel; (a) $L_{11}$ (b) $L_{12}$ (c) $L_{22}$. 
The Onsager diffusion coefficients determined from molecular simulations can be used in modeling carbon dioxide sequestration of organic nanopores of shale. The most significant opportunity of employing MD simulations for obtaining gas diffusivity in organic nanopores lies in areas where experiments for transport diffusivities are challenging. Measuring diffusivity at a wide range of loadings in the pores at extreme conditions such as infinite dilute loading and/or saturation loading is experimentally difficult. MD simulations can provide information about gas diffusion in organic pores under these conditions. Getting diffusivity data as a function of gas pressure is crucial to design carbon dioxide sequestration under a wide range of operating conditions.

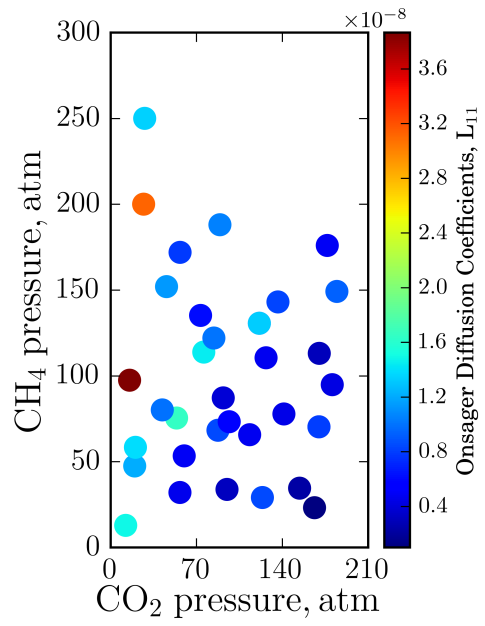

(a) $\mathrm{CH}_{4}-\mathrm{CH}_{4}$

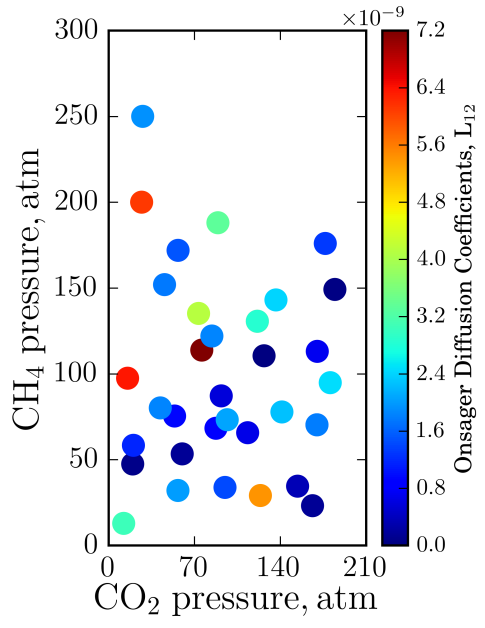

(b) $\mathrm{CH}_{4}-\mathrm{CO}_{2}$

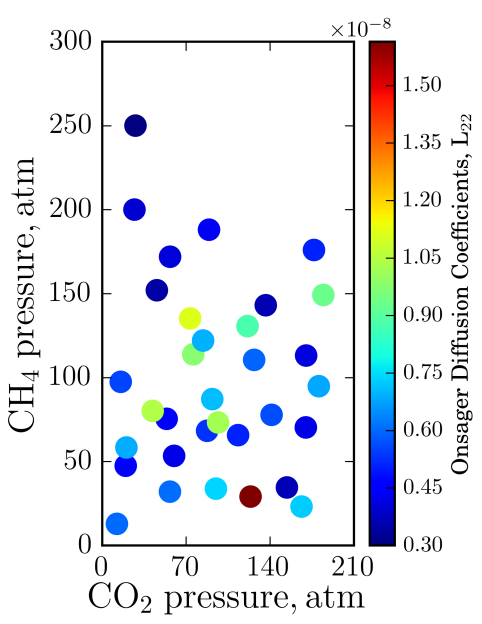

(c) $\mathrm{CO}_{2}-\mathrm{CO}_{2}$

Figure 4.12: Onsager diffusion coefficients of $\mathrm{CH}_{4}-\mathrm{CO}_{2}$ mixtures in the channel; (a) $L_{11}$ (b) $L_{12}$ (c) $L_{22}$. 


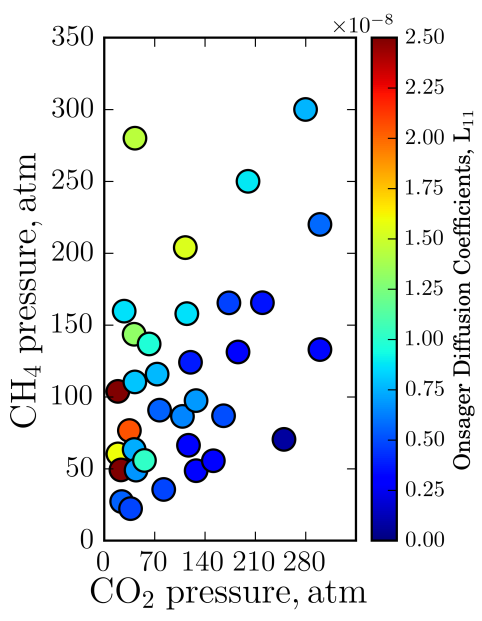

(a) $\mathrm{CH}_{4}-\mathrm{CH}_{4}$

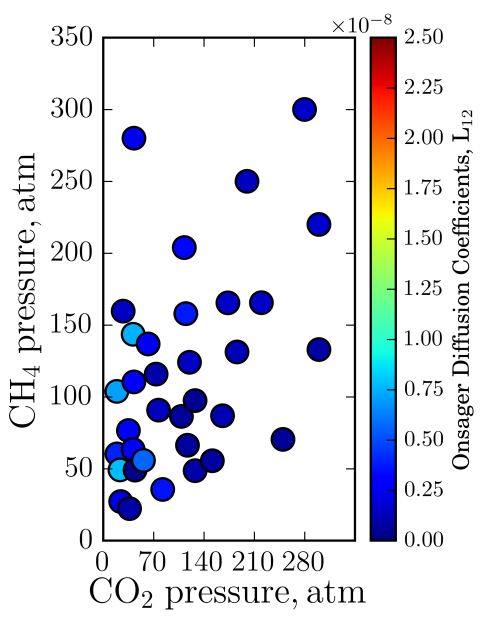

(b) $\mathrm{CH}_{4}-\mathrm{CO}_{2}$

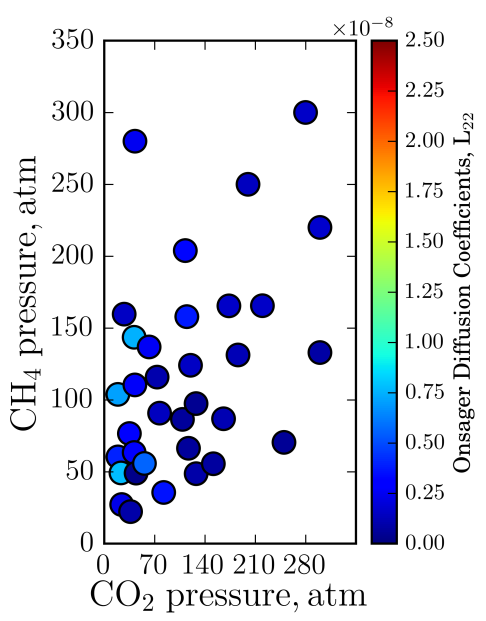

(c) $\mathrm{CO}_{2}-\mathrm{CO}_{2}$

Figure 4.13: Onsager diffusion coefficients of $\mathrm{CH}_{4}-\mathrm{CO}_{2}$ mixtures in the channel; (a) $L_{11}$ (b) $L_{12}$ (c) $L_{22}$.

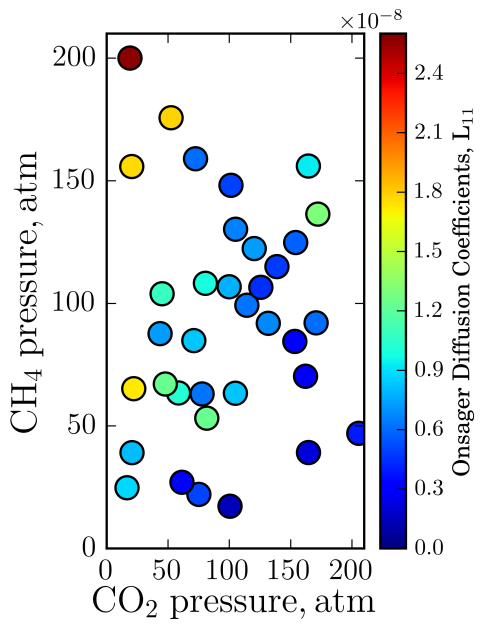

(a) $\mathrm{CH}_{4}-\mathrm{CH}_{4}$

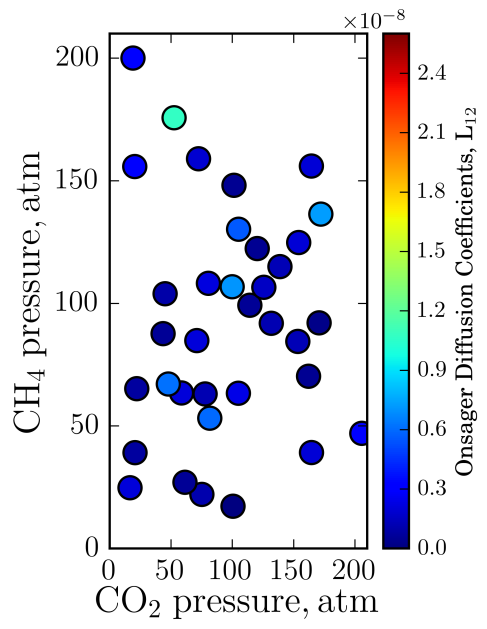

(b) $\mathrm{CH}_{4}-\mathrm{CO}_{2}$

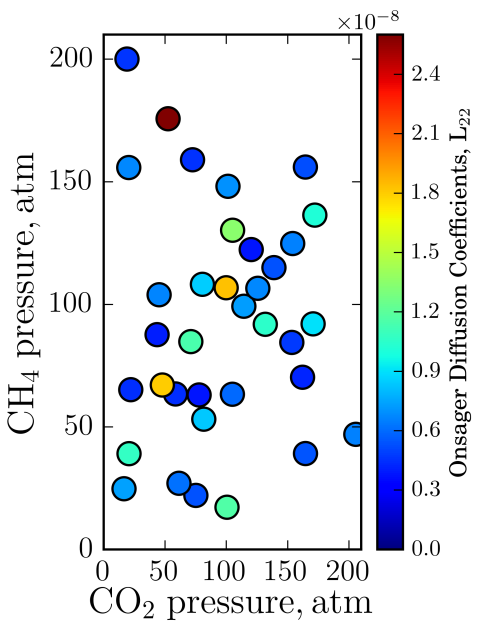

(c) $\mathrm{CO}_{2}-\mathrm{CO}_{2}$

Figure 4.14: Onsager diffusion coefficients of $\mathrm{CH}_{4}-\mathrm{CO}_{2}$ mixtures in the channel; (a) $L_{11}$ (b) $L_{12}$ (c) $L_{22}$. 


\section{Chapter 5}

\section{Storage and Transport in Realistic Kerogen Model}

Studying kerogen structure and its interactions with fluids is important for understanding the mechanisms involved in storage and production of hydrocarbons from shale. Kerogen is the source of hydrocarbons and the main constituents of the organic matter in sedimentary rocks $[76,88]$. The quantity and composition of the hydrocarbons accumulated during the kerogen evolution depend on its chemical composition and structural features. Kerogen is a mixture of organic chemicals and its chemical formula and properties vary depending on the geographical location [102]. Even when the chemical composition of kerogen is known, the molecular structure can vary depending on the pore space topology, porosity, and fluids present in the maturation process. This makes the modeling of kerogen challenging. Most of the current studies on the transport of fluids in organic-rich shale are mainly focused on fluid flow in simplified carbon-based conduits. The objective for this work is to determine the transport properties of multicomponent systems in kerogens of different maturities using molecular dynamics simulations. Kerogen types and structures are first discussed in section 5.1. In section 5.2, the method of creating a $3 \mathrm{D}$ solid structure of kerogen is presented. We also study the effect water and carbon dioxide on transport of hydrocarbons in organic nanopores. 


\section{$5.1 \quad$ Kerogen Types}

Three types of kerogens can be found in organic matter [7, 104]. These types differ from each other by the chemical content (carbon, oxygen, nitrogen, and sulfur content). Kerogen types are mainly defined by the percentage of elemental hydrogen present in them. Hydrogen content of immature kerogen decreases with thermal maturation [107]. Therefore, it can be used as a criteria to evaluate relative thermal maturity of kerogen [104]. After burial of kerogen in sedimentary rocks, it loses its hydrogen and oxygen content and becomes more concentrated in carbon content.

Type I kerogen has a highly aliphatic structure with a high $\mathrm{H} / \mathrm{C}$ ratio and a relatively low $\mathrm{O} / \mathrm{C}$ ratio. It is mainly originated from algal-lacustrine and algal-marine sources. Type II kerogen has a relatively high $\mathrm{H} / \mathrm{C}$ ratio with a low $\mathrm{O} / \mathrm{C}$ ratio. It is mainly originated from a mixture of sources from Type I and Type III. Type III kerogen has features similar to Vitrinites. It has a high O/C ratio with a relatively low $\mathrm{H} / \mathrm{C}$ ratio $[7,104]$. It is dominantly originated from higher plants accumulated in non-marine environments.

Due to high complexity of kerogen samples, it is never possible to propose a general kerogen model. Data are now available for different types of kerogens originated from different source rocks and many two dimensional (2D) models for kerogens are generated [7, 63, 93]. For validation of kerogen structures, experimental and theoretical results, such as mechanical properties and adsorption isotherms, should be compared. However, only using 3D structures, these properties can be calculated. In practice, the 2D structures can be converted to three dimensional (3D) structures using quantum mechanics and molecular dynamics methods [24, 78, 87]. The proposed 3D structures using computational techniques can provide wealth of information for comparisons of kerogens from different source rocks. Furthermore, they are helpful in predicting the mechanism in which the kerogen interacts with the bitumen and the mineral matter [45]. These 3D models also provide the functional groups of kerogen exposed to surface and interior functional groups hidden inside the kerogen [106]. Availability of a validated 3D model allows further computational study in order to predict the type of interaction between the kerogen and the mineral matrix, which is essential for oil production. Two approaches have been used to create 3D models from 2D models. First approach considers quantum mechanics calculations, such Gaussian optimization, for finding the most stable conformation (global maxima) [36]. This approach is not realistic for large systems (more than 500 atoms) $[29,39]$. In the second approach, the most stable conformations is found using molecular dynamics simulations [104]. In this work, Grand Canonical Monte Carlo (GCMC) and Equilibrium 
Molecular Dynamics (EMD) simulations are used to simulate the adsorption behavior and transport of methane in kerogens with different maturities.

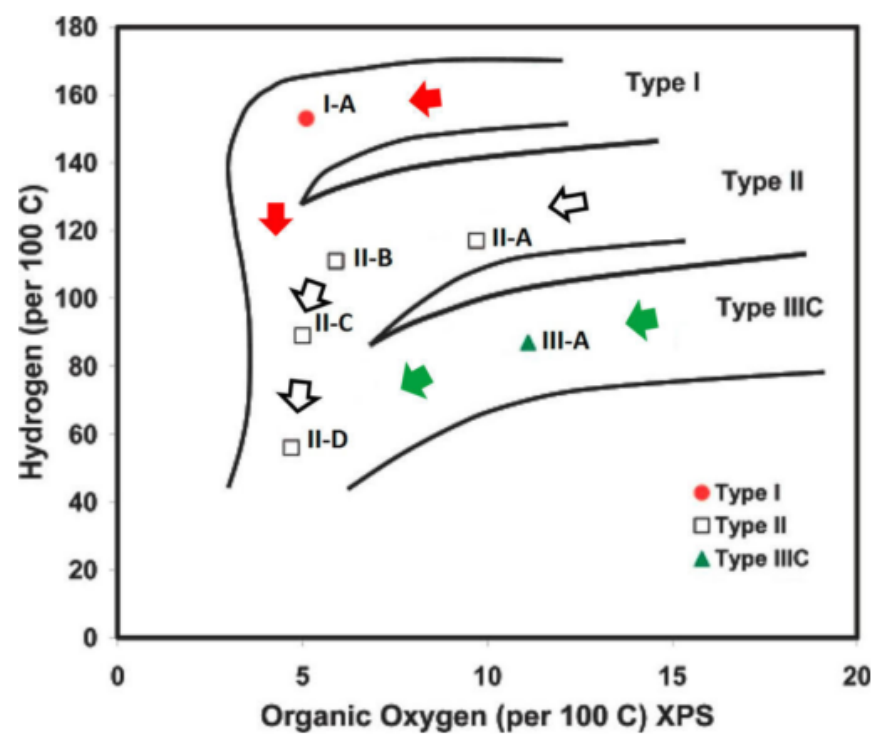

Figure 5.1: Van Krevelen diagram of the kerogen samples [60, 104]

\subsection{Creating Kerogen 3D structure}

Kerogen unit molecules with different maturities, prepared by Ungerer et al. [104] and available at [1], are used in this study. Since the purpose of this study is to investigate the storage and transport of gases, kerogen types IIA, IIB, IIC, IID, and IIIA are examined. The unit structures of each kerogen type are shown in Fig. 5.2. The molecular formulas of each kerogen is shown in Table. 5.1.

Table 5.1: Mass and chemical formula of kerogens with different maturities.

\begin{tabular}{c|c|l} 
Kerogen type & Unit cell chemical formula & Mass $(\mathrm{gr} / \mathrm{mol})$ \\
\hline \hline IIA & $C_{252} H_{294} N_{6} O_{24} S_{3}$ & 3887.252 \\
\hline IIB & $C_{224} H_{263} N_{5} O_{14} S_{2}$ & 3433.747 \\
\hline IIC & $C_{242} H_{219} N_{5} O_{13} S$ & 3469.484 \\
\hline IID & $C_{175} H_{102} N_{4} O_{9} S_{2}$ & 2468.834 \\
\hline IIIA & $C_{233} H_{204} N_{4} O_{27}$ & 3492.123
\end{tabular}

In order to build a representative solid state model of kerogen, eight kerogen molecules are placed in 


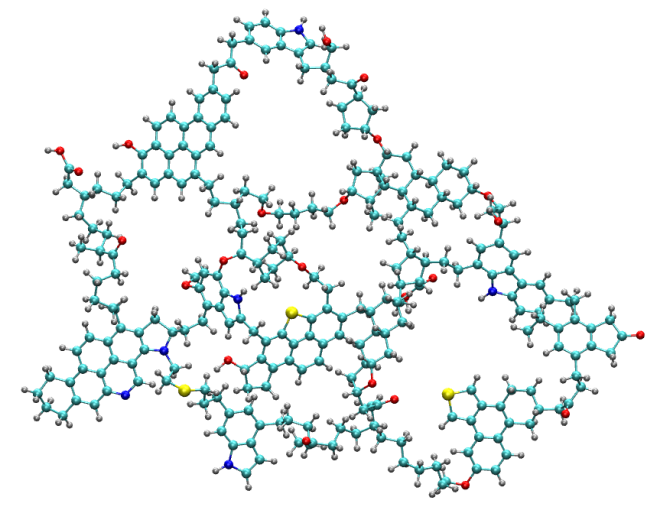

(a) Kerogen type IIA unit cell

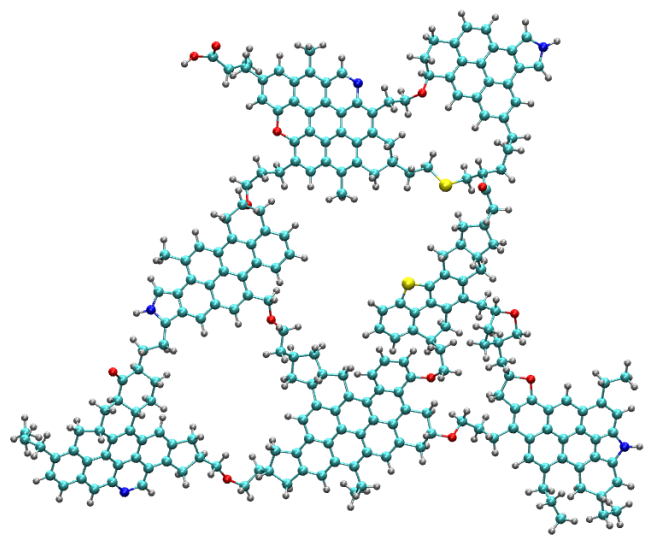

(c) Kerogen type IIC unit cell

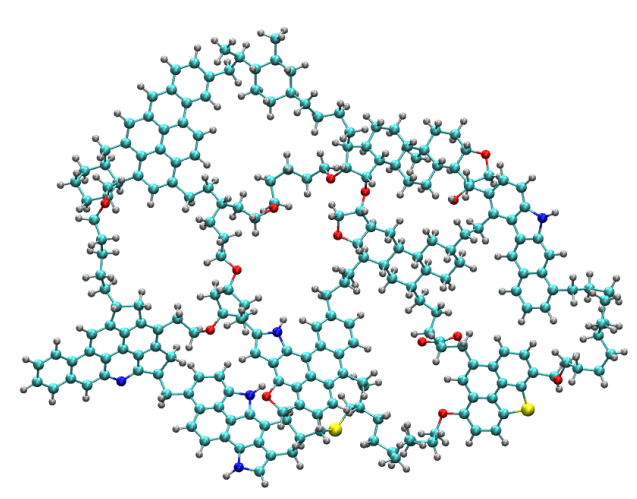

(b) Kerogen type IIB unit cell

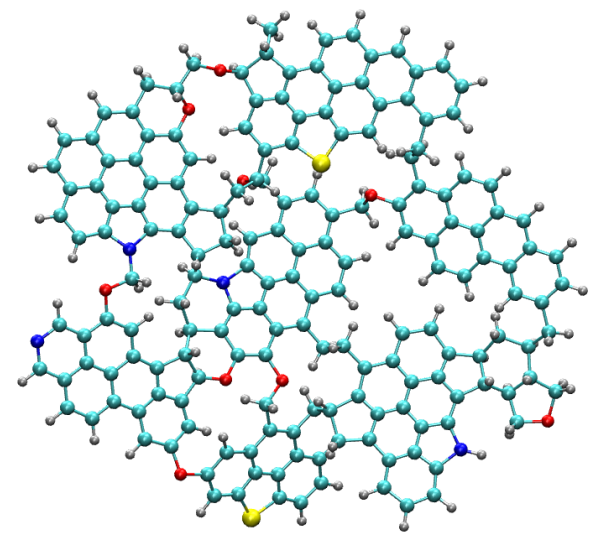

(d) Kerogen type IID unit cell

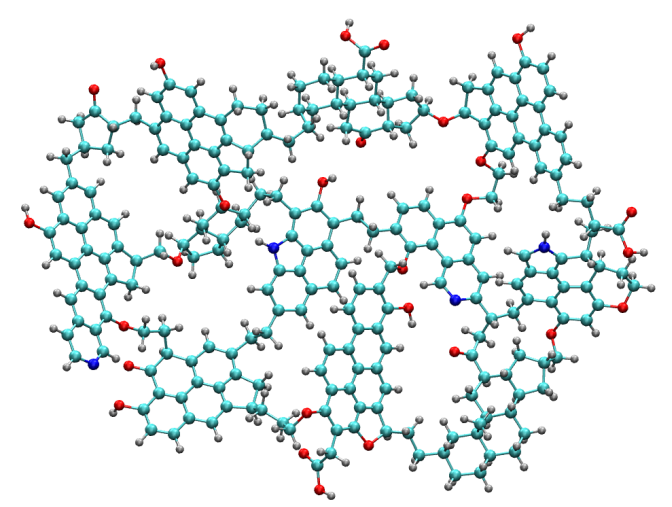

(e) Kerogen type IIIA unit cell

Figure 5.2: Unit cells of kerogen with different maturities. Color code: cyan is for carbon, silver for hydrogen, red for oxygen, blue for nitrogen, and yellow for sulfur.

a $10 \times 10 \times 10 \mathrm{~nm}$ periodic cubic cell using Moltemplate software [46]. The hydrocarbon molecules are not included in initial configurations. Instead, two spherical dummy particles are used to generate pore spaces in the $3 \mathrm{D}$ structures. The Lennard-Jones diameter of dummy particles is selected to be 15 ${ }^{\circ} A$. The dummy particles are deleted after 3D structure is developed. The COMPASS (Condensed- 
Phase Optimized Molecular Potentials for Atomistic Simulation Studies) class2 force field is used to describe the interactions between atoms, bonds, and angles [98]. Once the initial configurations of kerogen molecules are prepared, constant-temperature constant-volume (NVT) simulations are performed at $900 \mathrm{~K}$ for $3 \mathrm{~ns}$. Density of the system remains at $0.052,0.046,0.046,0.033,0.046$ $\mathrm{gr} / \mathrm{cm}^{3}$ for whole NVT simulation time for kerogen types IIA, IIB, IIC, IID, and IIIA, respectively. Three conformations are sampled from NVT simulations. Each sampled conformation goes under successive constant-temperature constant-pressure (NPT) simulations for a temperature range of 900 to $338 K$ at $20 \mathrm{MPa}$ pressure. Each NPT simulation is performed for $500 \mathrm{ps}$. All simulations are performed with $1 \mathrm{fs}$ timestep using LAMMPS molecular simulator [81].

Final structures of kerogen types IIA and IIB are shown in Fig. 5.3. Porosity of kerogen structures are calculated using the method described in Liang et al. [66]. Sizes of the kerogen structures and their porosities are presented in Table. 5.2.

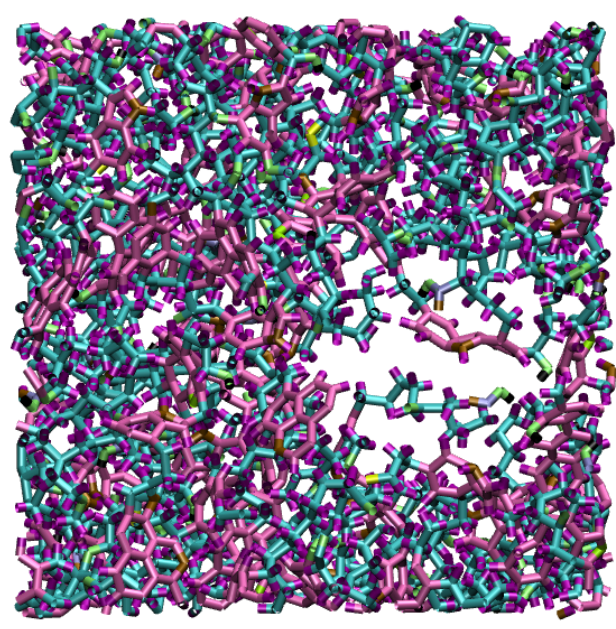

(a) Kerogen type IIA 3D structure

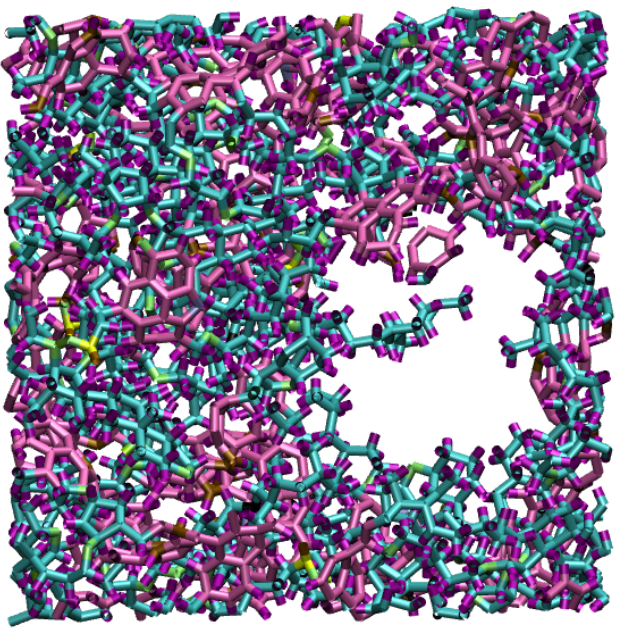

(b) Kerogen type IIB 3D structure

Figure 5.3: 3D structure of kerogen types IIA and IIB.

Table 5.2: Kerogen structures sizes and porosities.

\begin{tabular}{c|c|c|c} 
Kerogen type & Box Sizes & Porosity (\%) & Structure Density $\left(\mathrm{gr} / \mathrm{cm}^{3}\right)$ \\
\hline \hline IIA & $36.91 \times 36.91 \times 36.91$ & 13 & 1.03 \\
\hline IIB & $35.85 \times 35.85 \times 35.85$ & 15 & 1.01 \\
\hline IIC & $35.35 \times 35.35 \times 35.35$ & 16 & 1.02 \\
\hline IID & $31.43 \times 31.43 \times 31.43$ & 17 & 1.05 \\
\hline IIIA & $35.27 \times 35.27 \times 35.27$ & 16 & 1.06
\end{tabular}




\subsection{Molecular Simulation Results and Discussions}

In this section, the pure methane adsorption isotherms are determined and compared to experimental results. Furthermore, the multicomponent transport of gases inside these kerogen types are investigated.

\subsubsection{Adsorption Isotherms}

GCMC simulations are performed to determine the adsorption capability of kerogens with different maturities. For each maturity, initializing GCMC moves are used to place the methane molecules in the domain, minimizing the energy for a pressure range of 20 to $140 \mathrm{~atm}$. The adsorption isotherms are determined for each kerogen type and maturity (Fig. 5.4). Along with molecular simulation results, the experimental methane adsorption data of Rexer et al. [82] and Zhang et al. [112] are demonstrated. Rexer et al. [82] experimentally measured the sorption capabilities of bulk shale and isolated kerogens for immature, oil window, and gas window mature samples of Lower Toarcian Posidonia shale formation. The TOC of the samples range from 5.8-10.9 wt\%. The kerogen densities were measured using helium pycnometry and ranged from 1.024 to $1.368 \mathrm{gr} / \mathrm{cm}^{3}$. The geochemical data have shown that Posidonia kerogen is of type II. Two samples of Poidonia kerogen isotherms are used in Fig. 5.4. Kerogen types IIA and IID samples had vitrinite reflectance of 0.53 and $1.45 \%$, respectively. Methane isotherms were determined at $65^{\circ} \mathrm{C}$ (or $338 \mathrm{~K}$ ).

Zhang et al. [112] used samples from Eocene Green River Formation, Devonian-Mississippian Woodford Shale and Upper Cretaceous Cameo coal to study the methane adsorption at a temperature range of 35 to $65^{\circ} \mathrm{C}$. These formations have kerogen types of I, II, and III, respectively. In Fig. 5.4, the isotherm results of Woodford shale are only shown; however, Cameo coal kerogen isotherms had also a good match with our molecular simulation results. The Woodford shale sample had a vitrinite reflectance of $0.58 \%$, which is slightly higher than that for kerogen IIA in Rexer et al. [82].

The molecular simulation and experimental results are shown with hollow and solid markers, respectively. The Langmuir isotherm is defined as,

$$
q=q_{s a t} \frac{K p}{1+K p}
$$

where $q$ is absolute adsorption amount in $\mathrm{mmol} / \mathrm{gr}, q_{s a t}$ is maximum adsorption amount, $K$ is the 
equilibrium constant, and $p$ is pressure. The Langmuir isotherm is fitted to our adsorption results and plotted as solid lines in the same figure. The amount of adsorbed methane increases as pressure increases for all maturities. At a specific pressure, as the maturity of kerogens increases, the amount of adsorbed methane increases. Kerogen structures IID and IIIA have almost the same adsorbed amount and highest among other kerogen maturities. The adsorbed amount at 140 atm for kerogens type IID and IIIA are 1.25 and $1.28 \mathrm{mmol} / \mathrm{gr}$ TOC. The lowest adsorbed amount belongs to kerogen type IIA where it reaches to a value of $0.99 \mathrm{mmol} / \mathrm{gr}$ TOC at $140 \mathrm{~atm}$.

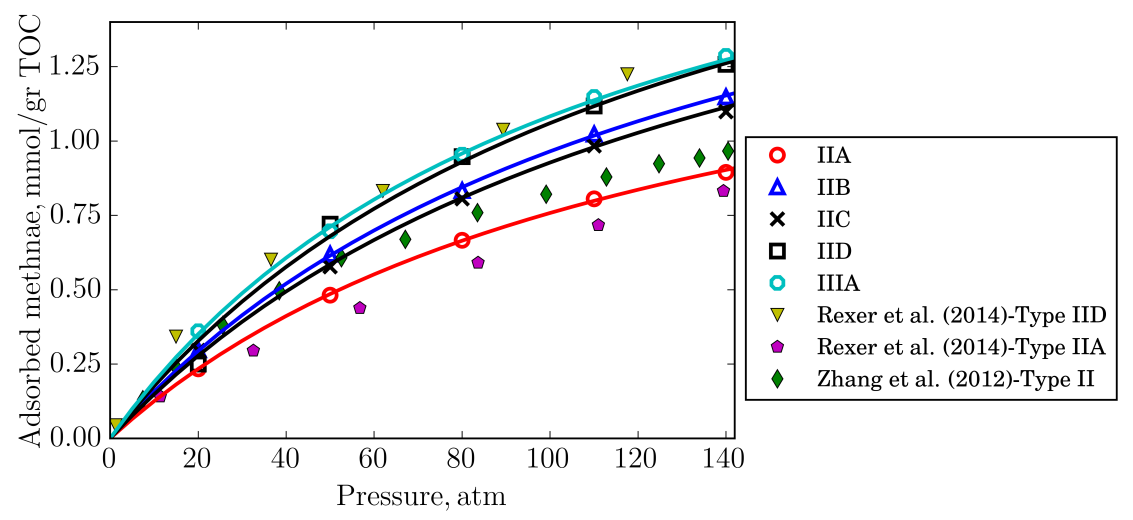

Figure 5.4: Adsorption isotherms of kerogens with different maturities along with experimental data of Rexer et al. [82] and Zhang et al. [112]

Experimental results of Zhang et al. [112] and Rexer et al. [82] also demonstrate the same trend for adsorption isotherms. For instance, kerogen type IID of Rexer et al. [82] has highest adsorbed amount and are well matched with our molecular simulation results. On the other hand, Rexer et al. [82] results for kerogen type IIA have lowest amount of adsorption where it reaches to 0.83 $\mathrm{mmol} / \mathrm{gr}$ TOC. The Zhang et al. [112] result on kerogen type II is within the range of our molecular simulation results and slightly higher than Rexer et al. [82] results. Furthermore, the adsorption isotherms are very well fitted to Langmuir isotherms. The Langmuir constants of Equation 5.1 are demonstrated in Table 5.3. The equilibrium constants are very similar for all kerogen types. However, the maximum adsorption amounts increases as kerogen becomes more mature. 
Table 5.3: The Langmuir constants for different kerogen maturities.

\begin{tabular}{c|c|c|c} 
Kerogen type & $q_{\text {sat }}, \mathrm{mmol} / \mathrm{kg}$ & $K, 1 / \mathrm{atm}$ & Goodness of fit, \% \\
\hline \hline IIA & 1.729 & 0.0078 & 99.96 \\
\hline IIB & 2.247 & 0.0075 & 99.96 \\
\hline IIC & 2.236 & 0.0071 & 99.87 \\
\hline IID & 2.404 & 0.0079 & 99.04 \\
\hline IIIA & 2.269 & 0.0091 & 99.89
\end{tabular}

The adsorption isotherms demonstrated the validity of our 3D kerogen structures and make it possible to study the transport phenomena in organic matters using created structures.

\subsubsection{Multicomponent Diffusion}

In transport of multicomponent gases, we are specifically interested in determining diffusion coefficients of methane as well as effect of presence of other components such as ethane, carbon dioxide, and water. To do that, two mixtures, one containing water and one without water, are introduced into kerogen structures with different maturities. The total number of molecules placed inside kerogen structure is 40. The composition of mixtures are shown in Table. 5.4. Methane is the major component of mixture with 70 and $74 \%$ mole fractions in Mixture 1 and 2, respectively. Ethane, water, and carbon dioxide each constitute 10 and $13 \%$ mole fraction of mixtures. The same species are used for all kerogen structures and simulations are performed at a temperature of $350 \mathrm{~K}$. In order to model water molecules, extended simple point charge (SPC/E) water model $[8,20]$ are employed. Methane and ethane molecules are modeled using OPLS-All atom force field [49]. The density of kerogen structures with Mixture 1 inside are 1.05, 1.02, 1.04, 1.11, and $1.09 \mathrm{gr} / \mathrm{cm}^{3}$ for kerogen types IIA, IIB, IIC, IID, and IIIA, respectively.

Table 5.4: Gas composition used for multicomponent diffusion.

\begin{tabular}{c|c|c} 
Gas & Mixture II Mole fraction, \% & Mixture II Mole fraction, \% \\
\hline \hline $\mathrm{CH}_{4}$ & 70 & 74 \\
\hline $\mathrm{CO}_{2}$ & 10 & 13 \\
\hline $\mathrm{C}_{2} \mathrm{H}_{6}$ & 10 & 13 \\
\hline $\mathrm{H}_{2} \mathrm{O}$ & 10 & 0
\end{tabular}


EMD simulations are performed and the Onsager diffusion coefficients are calculated using Equation. 4.4. The matrix of Onsager coefficients are plotted in Figs. 5.5, 5.6, 5.7, 5.8, and 5.9. The diagonal terms are also plotted in Fig. 5.10. Among diagonal diffusion terms, the diffusion coefficients for methane are one order of magnitude higher than those for other species in both mixtures. This is due to fact that the combination of higher adsorption strength and higher diffusion coefficients are rare [65], and therefore, since methane has lowest adsorption capability compared with other three components and therefore its Onsager diffusion coefficient $\left(\mathrm{L}_{\mathrm{CH}_{4}-\mathrm{CH}_{4}}\right)$ is highest among other gases. Onsager diffusion coefficients for methane are between 1.38 to $3.8 \times 10^{-9} \mathrm{~m}^{2} / \mathrm{s}$ for different kerogen types. Among other three gases, ethane has the highest diffusion coefficients and water shows lowest Onsager coefficients. The high adsorption capability of water is due to induced polarity of the surface functionalization that causes water molecules to be preferably be adsorbed to the surface of the pore over other species. Therefore, it causes the diffusion coefficients of water to be the lowest.

The off-diagonal diffusion coefficients are in the orders of magnitude as those of $\mathrm{CO}_{2}, \mathrm{C}_{2} \mathrm{H}_{6}$, and $\mathrm{H}_{2} \mathrm{O}$. There is no clear trend between the diagonal and off-diagonal terms as far as the kerogen becomes more mature. However, the off-diagonal diffusion coefficients of methane and any other species are highest compared with the other off-diagonal coefficients. The diffusion coefficients of hydrocarbons also reduces as the number of carbons in alkane chain increases.

The Onsager diffusion coefficients of all gases are higher Mixture I (containing water) compared to Mixture II (without water). Once water molecules are removed from pore space, the vacant adsorption sites become available for other species to be adsorbed. The vacant sites will be preferably filled by carbon dioxide molecules as it has higher adsorption capability compared to alkanes. It can be observed that the Onsager diffusion coefficients for carbon dioxide are lowest in Mixture II. The Onsager diffusion coefficients of other two alkanes (methane and ethane) are reduced in Mixture II compared with Mixture I as more adsorption sites are available in absence water. 


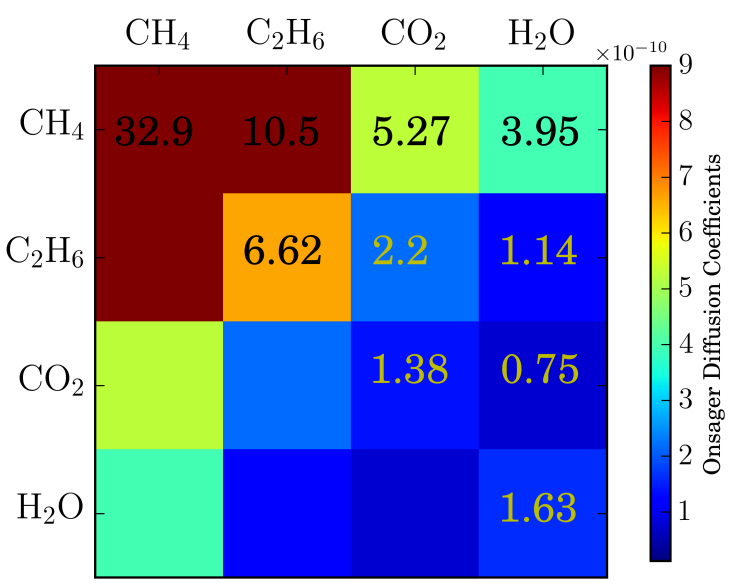

(a) Mixture containing water

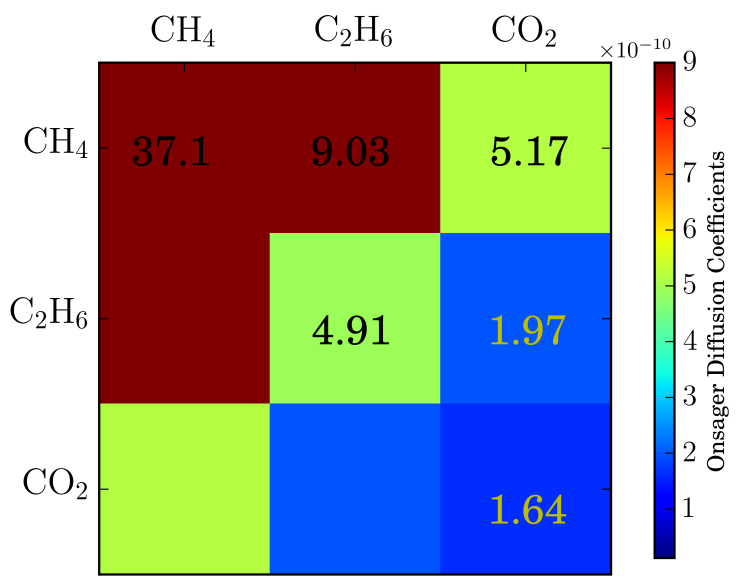

(b) Mixture without water

Figure 5.5: Matrices of Onsager coefficients for kerogen type IIA for both mixtures.

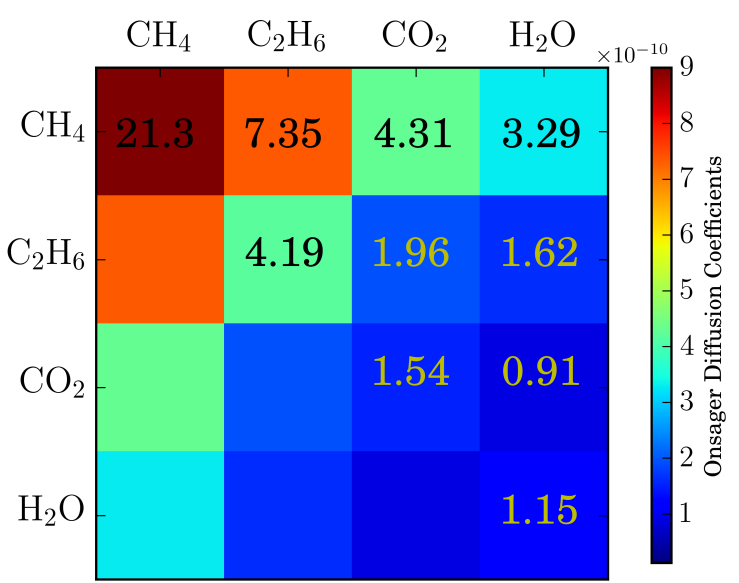

(a) Mixture containing water

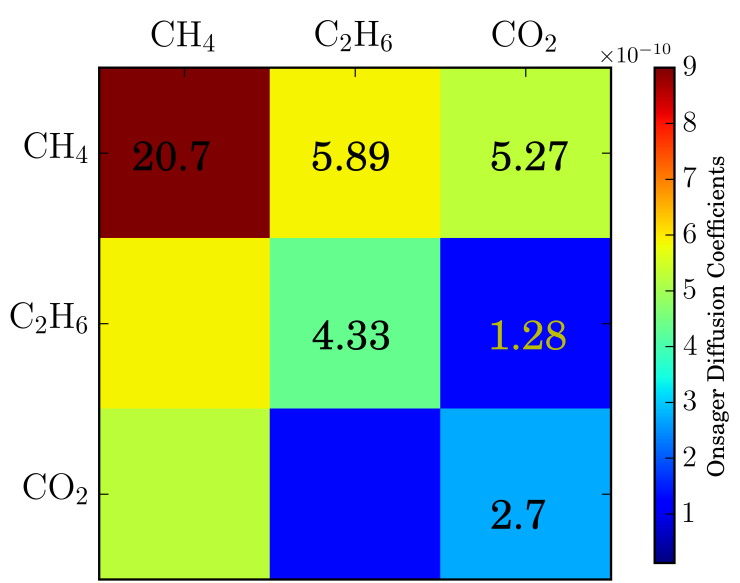

(b) Mixture without water

Figure 5.6: Matrices of Onsager coefficients for kerogen type IIB for both mixtures. 


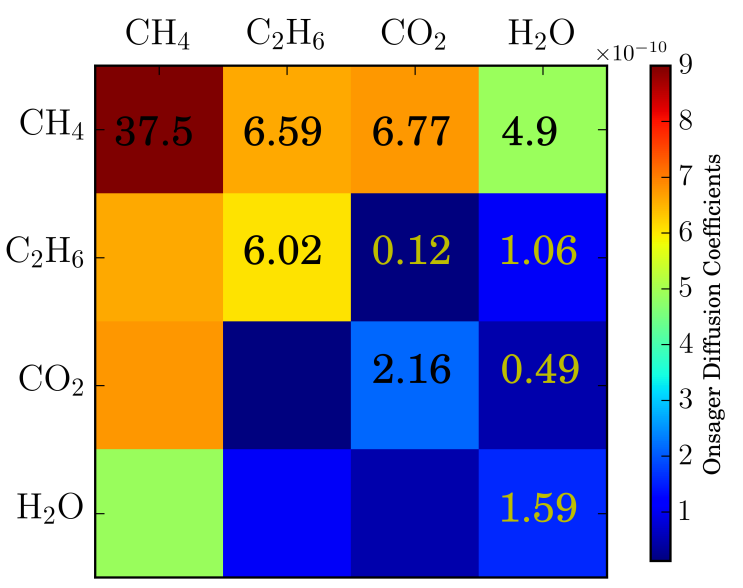

(a) Mixture containing water

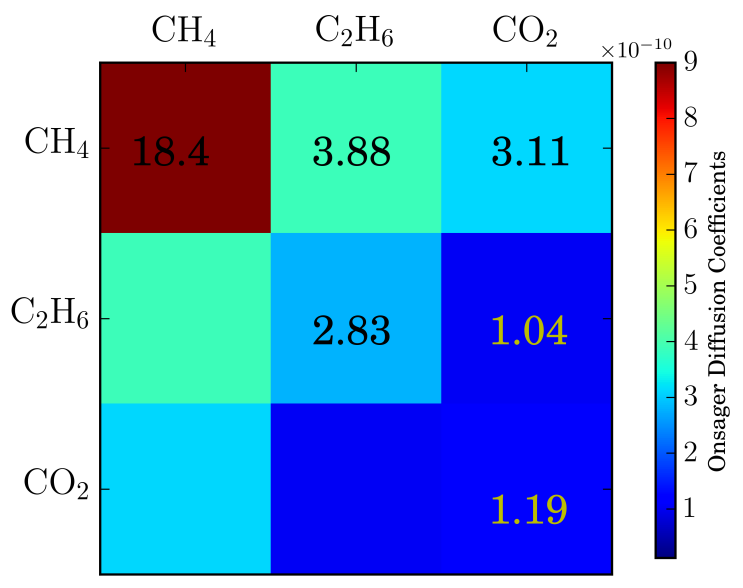

(b) Mixture without water

Figure 5.7: Matrices of Onsager coefficients for kerogen type IIC for both mixtures.

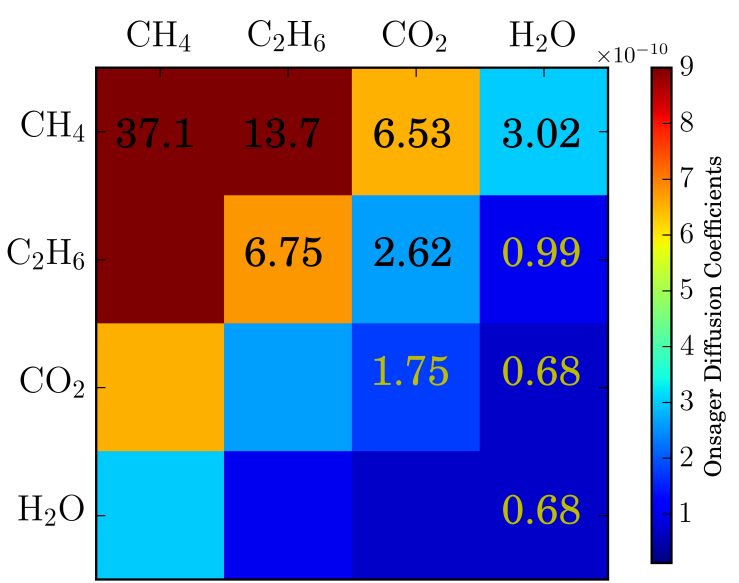

(a) Mixture containing water

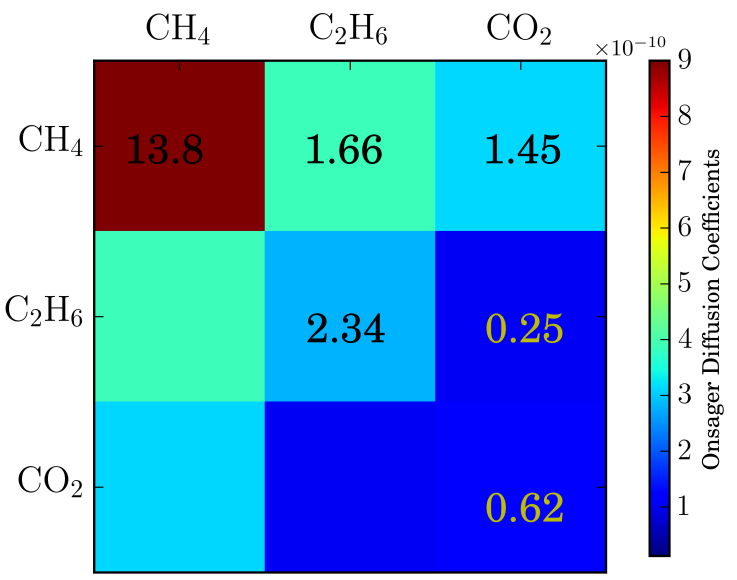

(b) Mixture without water

Figure 5.8: Matrices of Onsager coefficients for kerogen type IID for both mixtures. 


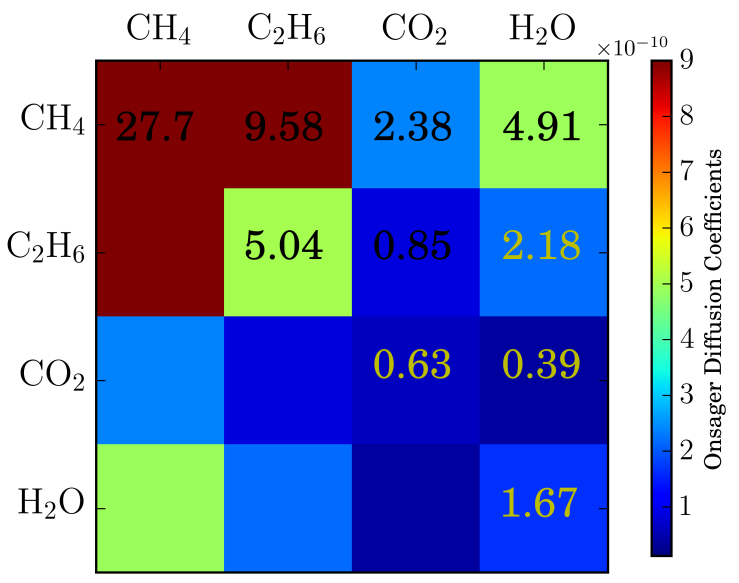

(a) Mixture containing water

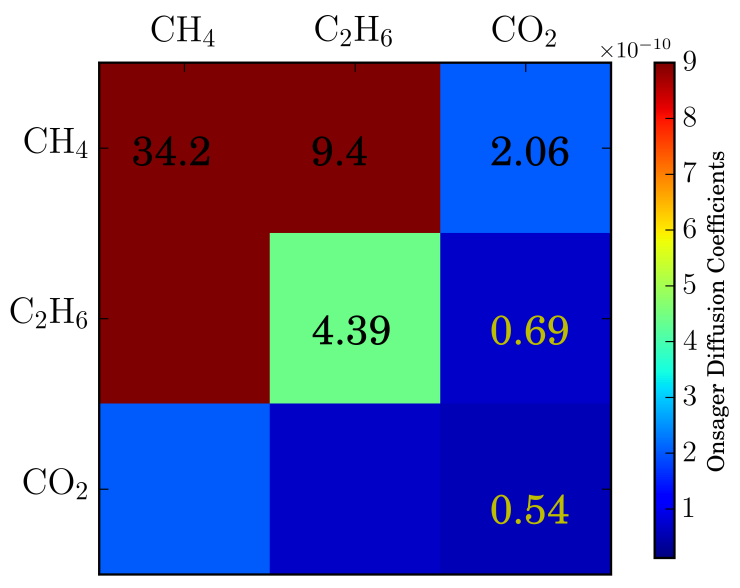

(b) Mixture without water

Figure 5.9: Matrices of Onsager coefficients for kerogen type IIIA for both mixtures.

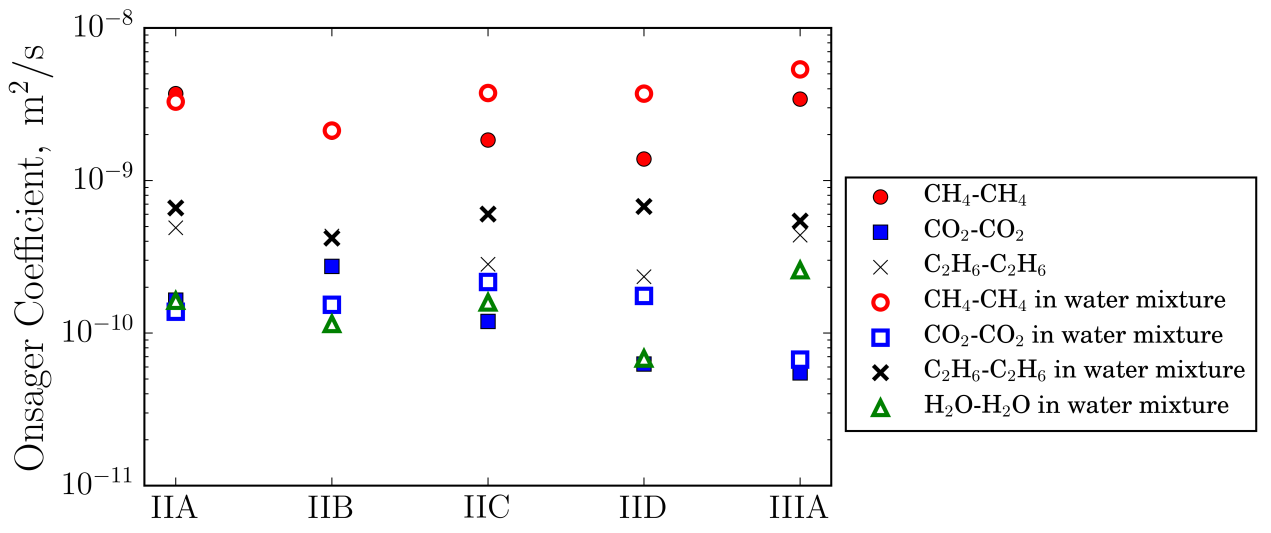

Figure 5.10: The diagonal components of Onsager matrix of diffusion coefficients determined using EMD simulations.

\subsubsection{Significance of Multicomponent Diffusion}

In order to better understand the significance of diagonal and off-diagonal Onsager diffusion coefficients, a simple numerical simulation is performed. We assumed that the numerical domain contains only kerogen type IIC. The schematic of the domain and its boundaries are demonstrated in Fig. 5.11. The domain is assumed to be 5 by $5 \mathrm{~mm}$. Three different boundaries are considered at corners of $2 \mathrm{D}$ square domain $\left(\partial \Omega_{1 i}, \partial \Omega_{1 j}\right.$, and $\left.\partial \Omega_{2}\right)$. Different concentrations are considered for the hydrocarbons (methane and ethane) at $\partial \Omega_{1 i}$ and $\partial \Omega_{1 j}$ boundaries while no concentration gradients are assumed for other two components (water and carbon dioxide). No flow boundary condition is 
assumed for all species at $\partial \Omega_{2}$. The goal of simulations is to investigate the diffusion of two species that do not have any concentration gradients. The concentration gradients of methane and ethane are in opposite directions and therefore a counter diffusion is expected to be observed for these two species.

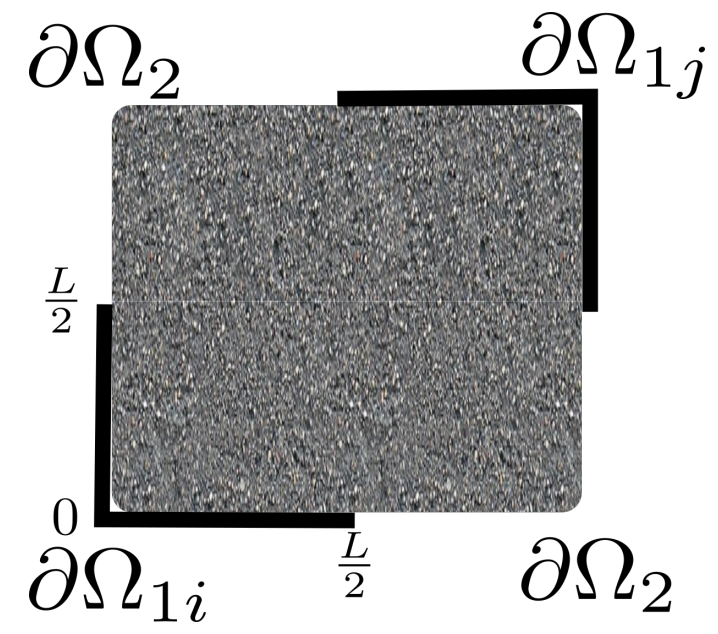

Figure 5.11: Schematic of simulation setup.

The diffusion equation needs to be solved is,

$$
\frac{\partial\left(c x_{i}\right)}{\partial t}+\nabla . J_{i}=0
$$

where,

$$
J_{i}=-c\left(\sum_{j=1}^{n-1} D_{i j} \nabla x_{j}\right)
$$

and the boundary conditions,

$$
\partial \Omega_{1 i}:\left\{\begin{array}{l}
x_{C_{H_{4}}}=0.72 \\
x_{C_{2} H_{6}}=0.08 \\
x_{C_{2}}=0.1 \\
x_{\mathrm{H}_{2} \mathrm{O}}=0.1
\end{array}\right.
$$




$$
\begin{gathered}
\partial \Omega_{1 j}:\left\{\begin{array}{l}
x_{\mathrm{CH}_{4}}=0.68 \\
x_{\mathrm{C}_{2} \mathrm{H}_{6}}=0.12 \\
x_{\mathrm{CO}_{2}}=0.1 \\
x_{\mathrm{H}_{2} \mathrm{O}}=0.1
\end{array}\right. \\
\partial \Omega_{2}: \text { No Flow Boundary }
\end{gathered}
$$

A $4 \%$ concentration gradients are considered for both methane and ethane while the concentration of two other components are kept constant. $D_{i j}$ in Equation 5.3 is the matrix of Fickian diffusion coefficients. The matrix of Onsager diffusion coefficients are determined from molecular simulations in previous section. In this part, we use molecular simulations results for kerogen type IIC. The Onsager diffusion coefficients are converted to those of Fickian ones using matrix of thermodynamic factors as,

$$
\left[\begin{array}{llll}
D_{11} & D_{12} & D_{13} & D_{14} \\
D_{21} & D_{22} & D_{23} & D_{24} \\
D_{31} & D_{32} & D_{33} & D_{34} \\
D_{41} & D_{42} & D_{43} & D_{44}
\end{array}\right]=\left[\begin{array}{cccc}
\Gamma_{11} & \Gamma_{12} & \Gamma_{13} & \Gamma_{14} \\
\Gamma_{21} & \Gamma_{22} & \Gamma_{23} & \Gamma_{24} \\
\Gamma_{31} & \Gamma_{32} & \Gamma_{33} & \Gamma_{34} \\
\Gamma_{41} & \Gamma_{42} & \Gamma_{43} & \Gamma_{44}
\end{array}\right]\left[\begin{array}{cccc}
L_{11} & L_{12} & L_{13} & L_{14} \\
L_{21} & L_{22} & L_{23} & L_{24} \\
L_{31} & L_{32} & L_{33} & L_{34} \\
L_{41} & L_{42} & L_{43} & L_{44}
\end{array}\right]
$$

where,

$$
L_{i j}=\frac{\Lambda_{i j}}{c_{j}}
$$

In the above equations, $\Lambda_{i j}$ is the Onsager diffusion coefficient, $\Gamma_{i j}$ is the thermodynamic factor, and $c_{j}$ is concentration of component $j$.

The concentration gradients of methane and ethane are selected to be small such that a constant thermodynamic factor and Onsager diffusion coefficients could be assumed. The result of methane simulations are shown in Fig. 5.12. We can see that the methane diffuse from higher concentration to lower concentration as expected. Similarly, for ethane, the gas diffuse from upper right corner of core to lower left corner (higher to lower concentration). The interesting results comes from other two gases that did not have any concentration gradients. While there are no concentration gradients for carbon dioxide and water, diffusion of these two species can be observed. This diffusion is more pronounced for carbon dioxide than for water. Their diffusion are due to cross-diffusivity coefficients 
(off-diagonal terms) of matrix of Fickian diffusion coefficients. This matrix for kerogen type IIC is shown in Fig. 5.16. The Fickian diffusion matrix is not symmetric. The cross diffusivity coefficients of methane with other species are also higher than other cross diffusivity coefficients.
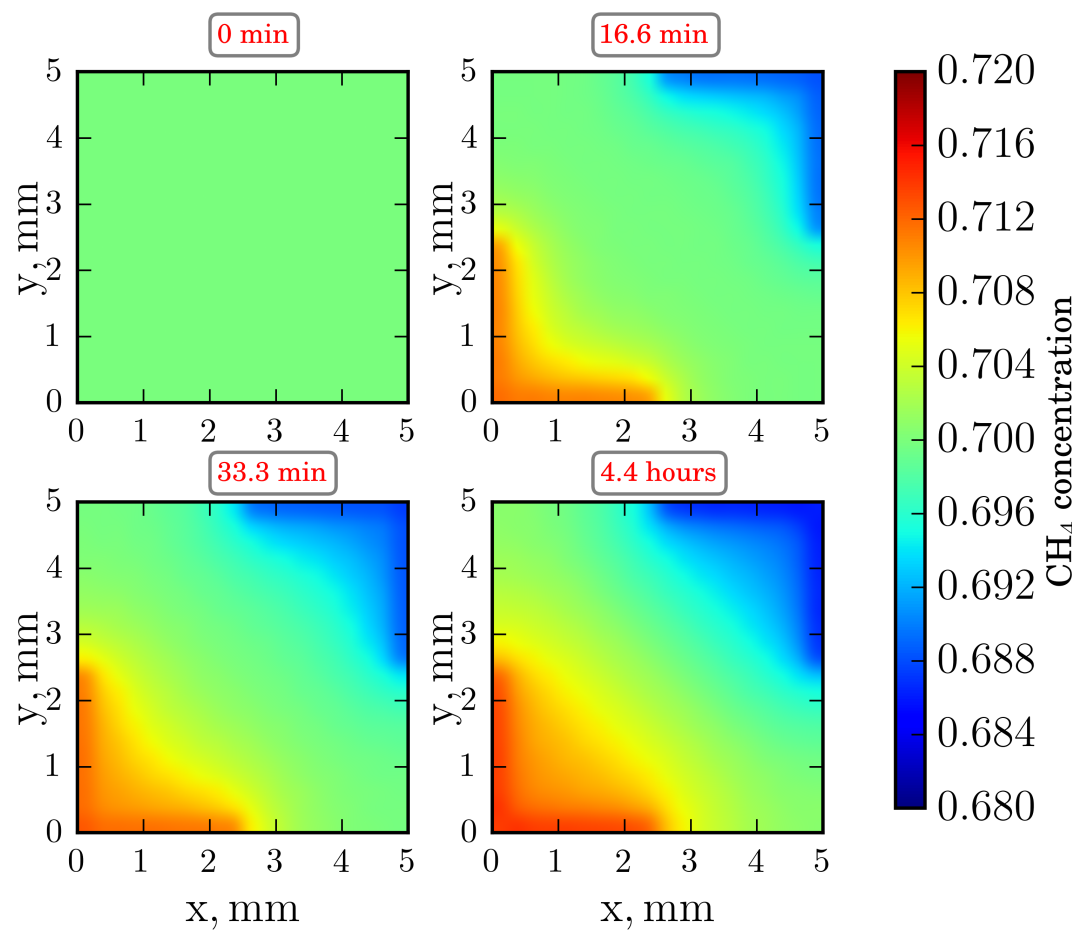

Figure 5.12: Diffusion of methane at different simulation times. 

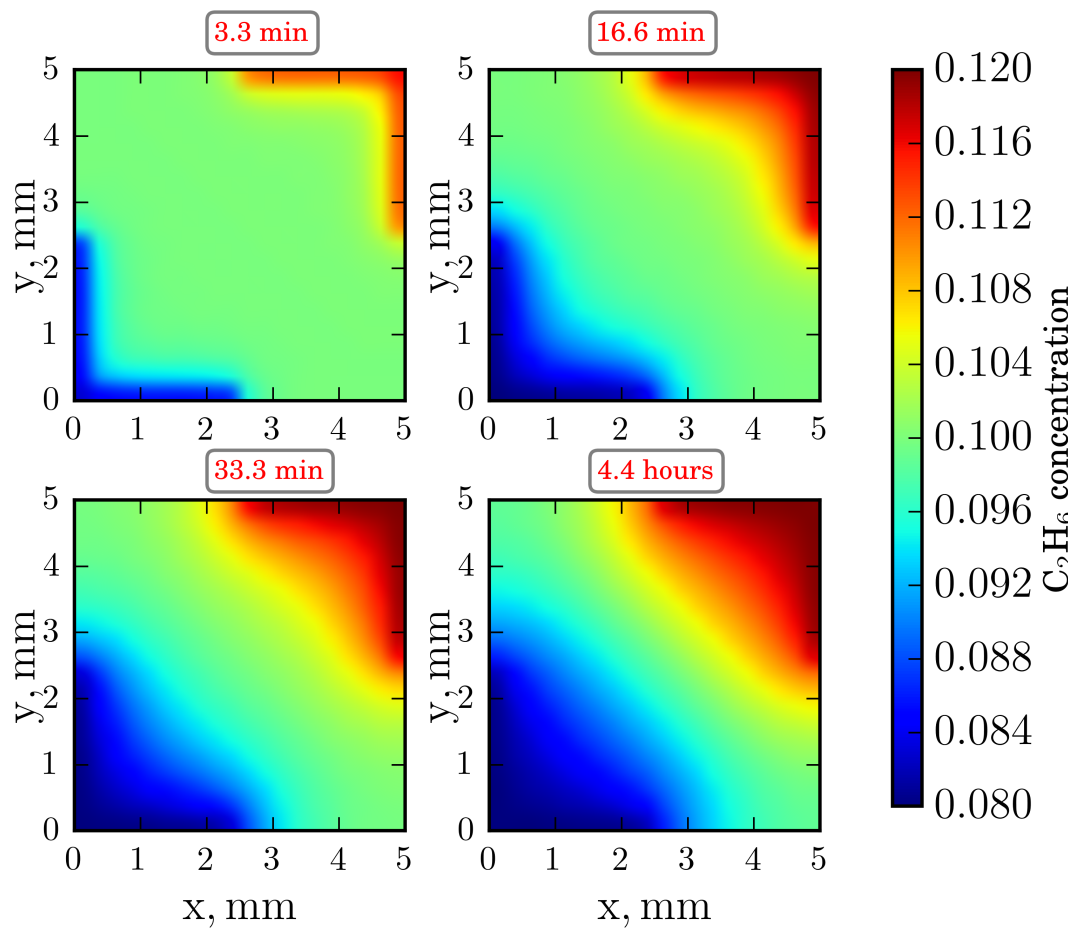

Figure 5.13: Diffusion of ethane at different simulation times.
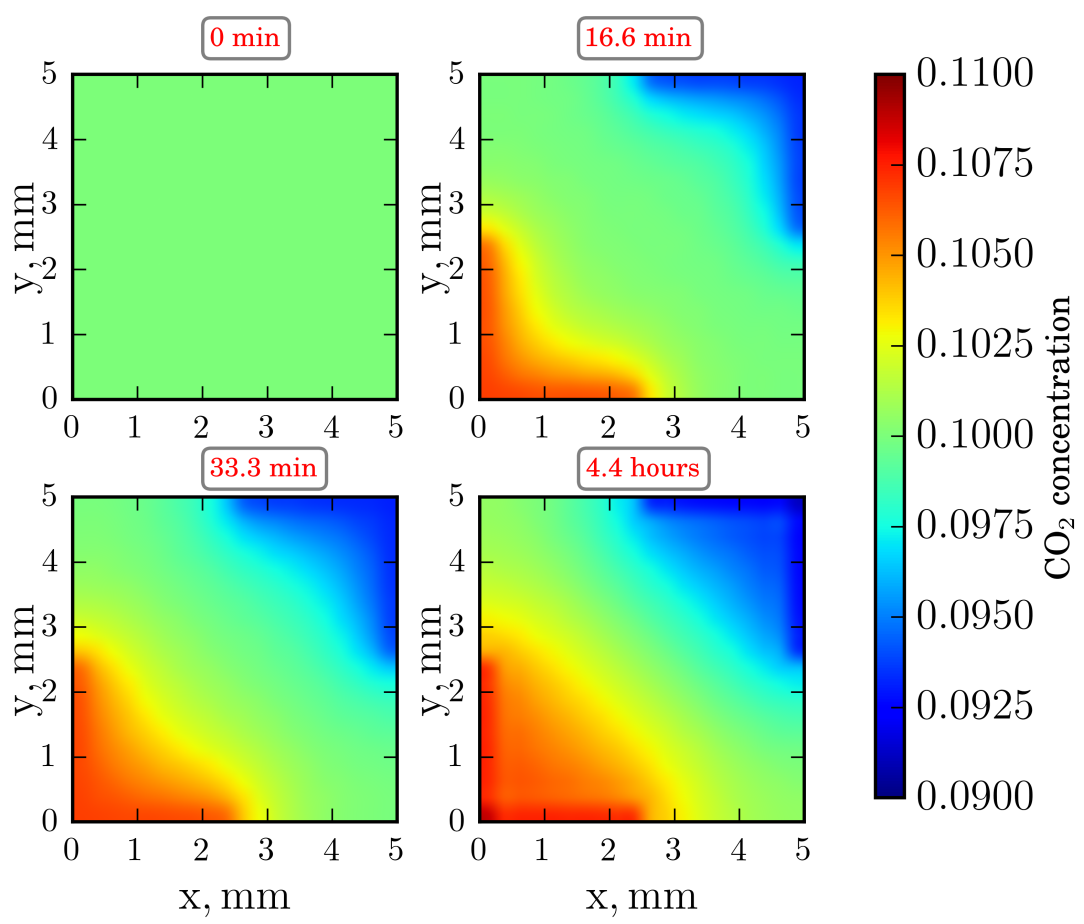

Figure 5.14: Diffusion of carbon dioxide at different simulation times. 

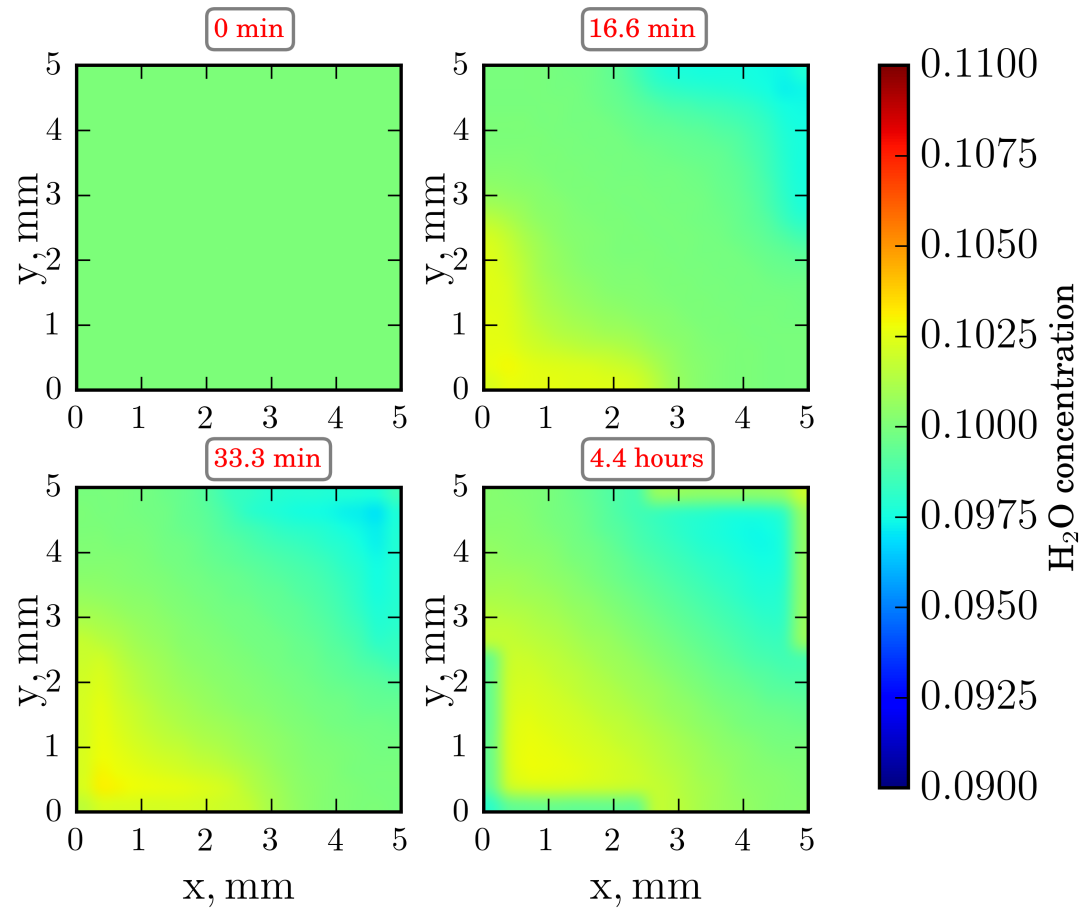

0.0950

0.0925

0.0900

Figure 5.15: Diffusion of water at different simulation times.

It is further observed that the directions of diffusion of carbon dioxide and water are along with diffusion of methane. Therefore, the cross diffusivity coefficients of carbon dioxide with methane which is highest among other cross diffusivities, cause carbon dioxide molecules diffusive from lower left corner to upper right corner. Same is also true for water. However, since the diffusion coefficients for water are smaller than those of carbon dioxide, smaller diffusion could be observed for water.

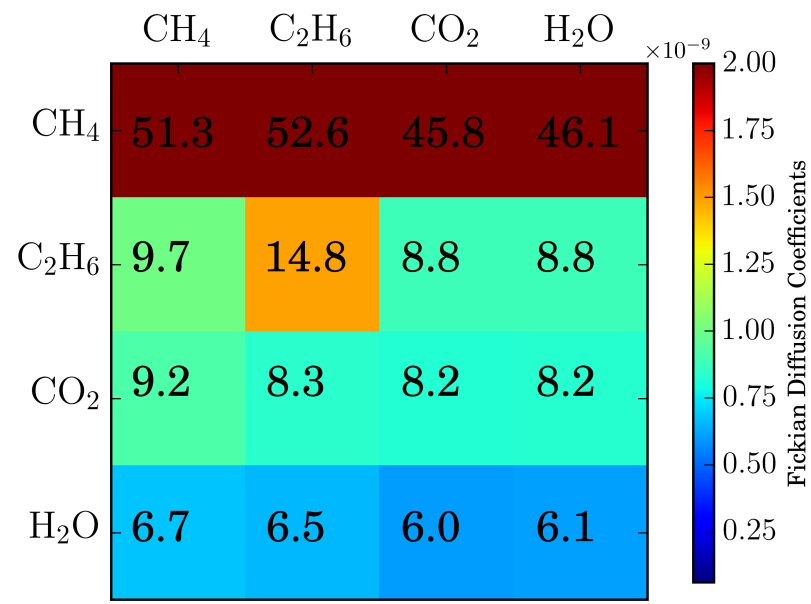

Figure 5.16: Fickian diffusion coefficients at initial conditions. 


\section{Chapter 6}

\section{Conclusions}

- Transport and adsorption of adsorbing and non-adsorbing gases are studied in organic nanopores of shale by performing molecular simulations in both simplified kerogen (organic nanochannel and nanotubes) and actual kerogen models (kerogen types IIA, IIB, IIC, IID, and IIIA).

- As the pressure increases, the amount of adsorbed gas molecules increases and tend to saturate at high pressures for both simplified and actual kerogens. For argon and methane, the adsorbed gas amount is higher compared to neon and helium. Excess adsorptions of argon and methane show an optimum pressure below which the excess adsorption increases and above it, the excess adsorption decreases.

- Plug-shaped velocity profiles are observed for all the gases and all the capillary sizes studied. Based on the computed mass fluxes, it is shown that the Knudsen diffusion model underestimates the molecular flux. This underestimation for flow of low adsorbing gases is less than that of high adsorbing gases. The thermodynamic factor in smaller capillaries are higher because molecules collide with capillary walls at higher rates compared with one another. Therefore, the differences between the transport and corrected transport diffusivity values are smaller for larger capillaries compared with those for smaller ones. It is found that less adsorptive gases have higher self-diffusion coefficient where neon demonstrate highest self-diffusivity. Viscosity of all gases reduces as pressure increases and saturates at high pressures. The viscosity of gases in nanopores are lower than their nominal values. It was found that the viscosity models for dilute gases under confinement are not good candidates to be used in organic nanopores due 
to adsorption of gases to pore surface. For adsorbing gases (with similar adsorbing properties) in a capillary, similar viscosity values are observed for each Knudsen number. The conclusions made in this study are drawn based on the MD simulation results in smooth structure-less geometries. Transport and adsorption of fluids on geometries with rough surfaces are different as follows.

- Multicomponent gas transport and adsorption in carbon-based organic channels with rough surfaces are investigated by performing non-equilibrium molecular dynamics simulations for two gas samples. Large portions of the heavier gas components are adsorbed to walls. Selectivity values of hexane, which is the heaviest gas component, compared to other gas components are higher than 1, which shows that it has the highest adsorption tendency. Therefore, in a organic nanopores of shale, the heavier components may remain in reservoir in adsorbed phase while the lighter components are produced.

In contrary to previous studies on single component gases flow in carbon-based smooth conduits, plug-shaped velocity profiles are not observed and the gas molecules velocities near the wall are smaller than other parts of channel. Lower velocities near the wall may be due to presence of rough surfaces and components heavier than methane.

Pressure in the $4 \mathrm{~nm}$ channel is lower than that in the $2 \mathrm{~nm}$ channel. Furthermore, at the same pressure gradient, the molecular flux values obtained in $4 \mathrm{~nm}$ channel is higher than those in the $2 \mathrm{~nm}$ channel. The rate of increase of molecular flux with pressure gradient parameter is higher for $4 \mathrm{~nm}$ channel.

The molecular flux of light composition sample is slightly higher than that of heavy composition sample in the $2 \mathrm{~nm}$ channel due to its higher composition of light molecules. Therefore, the heavier components reduce the molecular flux in the $2 \mathrm{~nm}$ channel, while their effect in the 4 nm channel is not significant. Furthermore, for both channels, diffusion coefficients decrease as the number of carbon atoms in the alkane chain increases (components become heavier).

- Kerogen structures with different maturity were created. The adsorption isotherms matches very well with experimental results. The Onsager diffusion coefficients of multicomponent gas flow were studied and and it was shown that the hydrocarbons have highest diffusion coefficients compared with water and carbon dioxide. The diffusion coefficients are also reduced as the number of carbons in alkane chain increases. Furthermore, hydrocarbons as well as 
carbon dioxide have higher diffusion coefficients in presence of water compared with a mixture containing no water.

- Carbon dioxide sequestration in organic nanopores of shale were investigated and binary adsorption isotherms as well as Onsager diffusion coefficients were determined. The Onsager diffusion coefficients determined from molecular simulations can be used in modeling carbon dioxide sequestration of organic nanopores of shale. The most significant opportunity of employing MD simulations for obtaining gas diffusivity in organic nanopores lies in areas where experiments for transport diffusivities are challenging. Measuring diffusivity at a wide range of loadings in the pores at extreme conditions such as infinite dilute loading and/or saturation loading is experimentally difficult. MD simulations can provide information about gas diffusion in organic pores under these conditions. Getting diffusivity data as a function of gas pressure is crucial to design carbon dioxide sequestration under a wide range of operating conditions. 


\section{Bibliography}

[1] Kerogen molecules coordinates and bonds information. http://www.materialsdesign.com/ science/structures/kerogens_and_coals. Accessed: 2016-09-30.

[2] Akkutlu, I. Y. and E. Fathi (2012). Multiscale gas transport in shales with local kerogen heterogeneities. SPE Journal 17(4), 1-002.

[3] Allen, M. P. et al. (2004). Introduction to molecular dynamics simulation. Computational soft matter: from synthetic polymers to proteins 23, 1-28.

[4] Arya, G., H. C. Chang, and E. J. Maginn (2001). A critical comparison of equilibrium, nonequilibrium and boundary-driven molecular dynamics techniques for studying transport in microporous materials. Journal of Chemical Physics 115(17), 8112-8124.

[5] Bahukudumbi, P. (2003). A unified engineering model for steady and quasi-steady shear-driven gas microflows. Microscale Thermophysical Engineering 7(4), 291-315.

[6] Barker, J., R. Fisher, and R. Watts (1971). Liquid argon: Monte carlo and molecular dynamics calculations. Molecular Physics 21(4), 657-673.

[7] Behar, F. and M. Vandenbroucke (1987). Chemical modelling of kerogens. Organic Geochemistry 11(1), 15-24.

[8] Berendsen, H., J. Grigera, T. Straatsma, et al. (1987). The missing term in effective pair potentials. J. phys. Chem 91(24), 6269-6271.

[9] Beskok, A. and G. E. Karniadakis (1999). Report: a model for flows in channels, pipes, and ducts at micro and nano scales. Microscale Thermophysical Engineering 3(1), 43-77. 
[10] Beskok, A., W. Trimmer, and G. E. Karniadakis (1996). Rarefaction and compressibility effects in gas microflows. Journal of Fluids Engineering 118(3), 448-456.

[11] Bhatia, S. K. and D. Nicholson (2003). Hydrodynamic origin of diffusion in nanopores. Physical review letters 90(1), 016105.

[12] Billemont, P., B. Coasne, and G. De Weireld (2013). Adsorption of carbon dioxide, methane, and their mixtures in porous carbons: effect of surface chemistry, water content, and pore disorder. Langmuir 29(10), 3328-3338.

[13] Bobylev, A. (1982). The chapman-enskog and grad methods for solving the boltzmann equation. In Akademiia Nauk SSSR Doklady, Volume 262, pp. 71-75.

[14] Bulatov, V. and W. Cai (2006). Computer simulations of dislocations.

[15] Carr, R., J. Comer, M. D. Ginsberg, and A. Aksimentiev (2011). Modeling pressure-driven transport of proteins through a nanochannel. Nanotechnology, IEEE Transactions on 10(1), $75-82$.

[16] Cercignani, C. (1969). Mathematical models in kinetic theory. Plenum Press.

[17] Cercignani, C. (1976, January). Theory and application of the Boltzmann equation. Elsevier.

[18] Chan, W. and Y. Sun (2003). Analytical modeling of ultra-thin-film bearings. Journal of Micromechanics and Microengineering 13(3), 463.

[19] Chapman, S. and T. G. Cowling (1970). The mathematical theory of non-uniform gases: an account of the kinetic theory of viscosity, thermal conduction and diffusion in gases. Cambridge university press.

[20] Chatterjee, S., P. G. Debenedetti, F. H. Stillinger, and R. M. Lynden-Bell (2008). A computational investigation of thermodynamics, structure, dynamics and solvation behavior in modified water models. The Journal of chemical physics 128(12), 124511.

[21] Choi, J.-G., D. D. Do, and H. D. Do (2001). Surface diffusion of adsorbed molecules in porous media: Monolayer, multilayer, and capillary condensation regimes. Industrial \& engineering chemistry research 40(19), 4005-4031. 
[22] Civan, F. (2010). Effective correlation of apparent gas permeability in tight porous media. Transport in Porous Media 82(2), 375-384.

[23] Civan, F., C. S. Rai, and C. H. Sondergeld (2010, October). Shale-gas permeability and diffusivity inferred by improved formulation of relevant retention and transport mechanisms. Transport in Porous Media 86(3), 925-944.

[24] Collell, J., G. Galliero, F. Gouth, F. Montel, M. Pujol, P. Ungerer, and M. Yiannourakou (2014). Molecular simulation and modelisation of methane/ethane mixtures adsorption onto a microporous molecular model of kerogen under typical reservoir conditions. Microporous and Mesoporous Materials 197, 194-203.

[25] Collell, J., G. Galliero, R. Vermorel, P. Ungerer, M. Yiannourakou, F. Montel, and M. Pujol (2015). Transport of Multicomponent Hydrocarbon Mixtures in Shale Organic Matter by Molecular Simulations. Journal of Physical Chemistry C 119(39), 22587-22595.

[26] Comets, F., S. Popov, G. M. Schütz, and M. Vachkovskaia (2010). Knudsen gas in a finite random tube: transport diffusion and first passage properties. Journal of Statistical Physics 140(5), $948-984$.

[27] Darabi, H., A. Ettehad, F. Javadpour, and K. Sepehrnoori (2012). Gas flow in ultra-tight shale strata. Journal of Fluid Mechanics 710, 641-658.

[28] Dullien, F. (1963). New relationship between viscosity and the diffusion coefficients based on lamm's theory of diffusion. Transactions of the Faraday Society 59, 856-868.

[29] Dupuis, M., J. Watts, H. Villar, and G. Hurst (1989). The general atomic and molecular electronic structure system hondo: version 7.0. Computer physics communications 52(3), 415425.

[30] Feng, F. and I. Y. Akkutlu (2015). Flow of Hydrocarbons in Nanocapillary : A Non-Equilibrium Molecular. In SPE Asia Pacific Unconventional Resources Conference and Exhibition held in Brisbane, Australia, pp. 1-18.

[31] Feng, F., I. Y. Akkutlu, et al. (2015). Flow of hydrocarbons in nanocapillary: A non-equilibrium molecular dynamics study. In SPE Asia Pacific Unconventional Resources Conference and Exhibition. Society of Petroleum Engineers. 
[32] Firouzi, M., K. Alnoaimi, A. Kovscek, and J. Wilcox (2014, mar). Klinkenberg effect on predicting and measuring helium permeability in gas shales. International Journal of Coal Geology 123, $62-68$.

[33] Firouzi, M. and J. Wilcox (2012). Molecular modeling of carbon dioxide transport and storage in porous carbon-based materials. Microporous and Mesoporous Materials 158, 195-203.

[34] Florence, F. A., J. Rushing, K. E. Newsham, T. A. Blasingame, et al. (2007). Improved permeability prediction relations for low permeability sands. In Rocky Mountain Oil \& Gas Technology Symposium. Society of Petroleum Engineers.

[35] Grad, H. (1949). On the kinetic theory of rarefied gases. Communications on pure and applied mathematics 2(4), 331-407.

[36] Guan, X.-H., Y. Liu, D. Wang, Q. Wang, M.-S. Chi, S. Liu, and C.-G. Liu (2015). Threedimensional structure of a huadian oil shale kerogen model: An experimental and theoretical study. Energy \&3 Fuels 29(7), 4122-4136.

[37] He, S., Y. Ning, T. Chen, H. Liu, H. Wang, and G. Qin (2016). Estimation of Transport Diffusivity of Natural Gas in Organic Matter Using. In SPE Low Perm Symposium, Denver, CO.

[38] Heffelfinger, G. S. and F. van Swol (1994). Diffusion in lennard-jones fluids using dual control volume grand canonical molecular dynamics simulation (dcv-gcmd). The Journal of chemical physics 100(10), 7548-7552.

[39] Heller, E. (2006). Guided gaussian wave packets. Accounts of chemical research 39(2), 127-134.

[40] Heller, R. and M. Zoback (2014). Adsorption of methane and carbon dioxide on gas shale and pure mineral samples. Journal of Unconventional Oil and Gas Resources 8, 14-24.

[41] Hippler, H., V. Schubert, and J. Troe (1984). Photolysis quantum yields and atom recombination rates of bromine in compressed gases. experiments up to 7 kbar. The Journal of chemical physics 81(9), 3931-3941.

[42] Holt, J. K., H. G. Park, Y. Wang, M. Stadermann, A. B. Artyukhin, C. P. Grigoropoulos, A. Noy, and O. Bakajin (2006). Fast mass transport through sub-2-nanometer carbon nanotubes. Science 312(5776), 1034-1037. 
[43] Humphrey, W., A. Dalke, and K. Schulten (1996). Vmd: visual molecular dynamics. Journal of molecular graphics 14(1), 33-38.

[44] Javadpour, F. (2009). Nanopores and apparent permeability of gas flow in mudrocks (shales and siltstone). Journal of Canadian Petroleum Technology 48(8), 16-21.

[45] Jeong, K. and T. Kobylinski (1983). Organic-mineral matter interactions in green river oil shale. Prepr. Pap.-Am. Chem. Soc., Div. Fuel Chem.;(United States) 28(CONF-830303-).

[46] Jewett, A. I., Z. Zhuang, and J.-E. Shea (2013). Moltemplate a coarse-grained model assembly tool. Biophysical Journal 104(2), 169a.

[47] Jin, Z. and A. Firoozabadi (2015). Flow of methane in shale nanopores at low and high pressure by molecular dynamics simulations. The Journal of chemical physics 143(10), 104315.

[48] Jones, F. O., W. Owens, et al. (1980). A laboratory study of low-permeability gas sands. Journal of Petroleum Technology 32(09), 1-631.

[49] Jorgensen, W. L., D. S. Maxwell, and J. Tirado-Rives (1996). Development and testing of the opls all-atom force field on conformational energetics and properties of organic liquids. Journal of the American Chemical Society 118(45), 11225-11236.

[50] Kang, S. M., E. Fathi, R. J. Ambrose, I. Y. Akkutlu, and R. F. Sigal (2011). Carbon Dioxide Storage Capacity of Organic-rich Shales. SPE Journal (December), 842-855.

[51] Kärger, J., D. M. Ruthven, and D. N. Theodorou (2012). Diffusion in nanoporous materials. John Wiley \& Sons.

[52] Karniadakis, G., A. Beskok, N. R. Aluru, et al. (2006). Microflows and nanoflows: fundamentals and simulation, Volume 29. Springer.

[53] Karniadakis, G. E., A. Beskok, and N. Aluru (2006). Microflows and nanoflows: fundamentals and simulation, Volume 29. Springer Science \& Business Media.

[54] Kazamierkiewicz, R. Introduction to molecular modelling. Intercollegiate Faculty of Biotechnology $U G-M U G$.

[55] Kazemi, M. and A. Takbiri-Borujeni (2015a). An analytical model for shale gas permeability. International Journal of Coal Geology 146, 188-197. 
[56] Kazemi, M. and A. Takbiri-Borujeni (2015b). Predicting gas apparent permeability of shale samples: A novel analytical approach. In SPE Annual Technical Conference and Exhibition. Society of Petroleum Engineers.

[57] Kazemi, M. and A. Takbiri-Borujeni (2016a). Flow of gases in nanocapillary organic nanopores of shale: A boundary-driven molecular simulation study. In SPE Western Regional Meeting. Society of Petroleum Engineers.

[58] Kazemi, M. and A. Takbiri-Borujeni (2016b). Flow of gases in organic nanoscale channels: A boundary-driven molecular simulation study. Energy \& Fuels.

[59] Kazemi, M. and A. Takbiri-Borujeni (2016c). Non-equilibrium molecular dynamics simulation of gas flow in organic nanochannels. Journal of Natural Gas Science and Engineering.

[60] Kelemen, S., M. Afeworki, M. Gorbaty, M. Sansone, P. Kwiatek, C. Walters, H. Freund, M. Siskin, A. Bence, D. Curry, et al. (2007). Direct characterization of kerogen by x-ray and solid-state 13c nuclear magnetic resonance methods. Energy \&3 Fuels 21(3), 1548-1561.

[61] Keller, J. U. and R. Staudt (2005). Gas adsorption equilibria: experimental methods and adsorptive isotherms. Springer Science \& Business Media.

[62] Khosrokhavar, R., K.-H. Wolf, and H. Bruining (2014). Sorption of ch 4 and co 2 on a carboniferous shale from belgium using a manometric setup. International Journal of Coal Geology 128, $153-161$.

[63] Kidena, K., S. Murata, and M. Nomura (2008). A newly proposed view on coal molecular structure integrating two concepts: Two phase and uniphase models. Fuel Processing Technology 89(4), $424-433$.

[64] Klinkenberg, L. (1941). The permeability of porous media to liquids and gases. In Drilling and Production Practice, pp. 200-213. American Petroleum Institute.

[65] Krishna, R. and J. Van Baten (2008). Insights into diffusion of gases in zeolites gained from molecular dynamics simulations. Microporous and Mesoporous Materials 109(1), 91-108.

[66] Liang, J., H. Edelsbrunner, P. Fu, P. V. Sudhakar, and S. Subramaniam (1998). Analytical shape computation of macromolecules: Ii. inaccessible cavities in proteins. Proteins Structure Function and Genetics 33(1), 18-29. 
[67] Loucks, R. G., R. M. Reed, S. C. Ruppel, and U. Hammes (2012). Spectrum of pore types and networks in mudrocks and a descriptive classification for matrix-related mudrock pores. $A A P G$ bulletin 96(6), 1071-1098.

[68] Ma, Y. and A. Jamili (2016). Modeling the density profiles and adsorption of pure and mixture hydrocarbons in shales. Journal of Unconventional Oil and Gas Resources 14, 128-138.

[69] Martin, M. G. and J. I. Siepmann (1998). Transferable potentials for phase equilibria. 1. unitedatom description of n-alkanes. The Journal of Physical Chemistry B 102(14), 2569-2577.

[70] Mason, E. A. and A. Malinauskas (1983). Gas transport in porous media: the dusty-gas model. Elsevier Science Ltd.

[71] Mavor, M., L. Owen, and T. Pratt (1990). Measurement and evaluation of coal sorption isotherm data. In SPE Annual Technical Conference and Exhibition, New Orleans, Louisiana.

[72] Michalis, V. K., A. N. Kalarakis, E. D. Skouras, and V. N. Burganos (2010). Rarefaction effects on gas viscosity in the Knudsen transition regime. Microfluidics and Nanofluidics 9(4-5), 847-853.

[73] Mosher, K., Y. Liu, and J. Wilcox (2011). The Impact of Pore Size On Methane and CO2 Adsorption In Carbon. Ph. D. thesis, Stanford University.

[74] Müser, M. and B. Berne (1996). Path-integral monte carlo scheme for rigid tops: Application to the quantum rotator phase transition in solid methane. Physical review letters $77(13), 2638$.

[75] Nagy, J., D. F. Weaver, and V. H. Smith Jr (1995). Ab initio methane dimer intermolecular potentials. Molecular Physics 85(6), 1179-1192.

[76] Nomura, M., L. Artok, S. Murata, A. Yamamoto, H. Hama, H. Gao, and K. Kidena (1998). Structural evaluation of zao zhuang coal. Energy $\&$ fuels 12(3), 512-523.

[77] Nuttall, B. C., C. F. Eble, J. A. Drahovzal, and R. M. Bustin (2005). Analysis of Devonian black shales in Kentucky for potential carbon dioxide sequestration and enhanced natural gas production. Kentucky Geological Survey Report DE-FC26-02NT41442.

[78] Orendt, A. M., I. S. Pimienta, S. R. Badu, M. S. Solum, R. J. Pugmire, J. C. Facelli, D. R. Locke, K. W. Chapman, P. J. Chupas, and R. E. Winans (2013). Three-dimensional structure 
of the siskin green river oil shale kerogen model: a comparison between calculated and observed properties. Energy \&3 Fuels 27(2), 702-710.

[79] Ottiger, S., R. Pini, G. Storti, and M. Mazzotti (2008). Competitive adsorption equilibria of co2 and ch4 on a dry coal. Adsorption 14(4-5), 539-556.

[80] Pini, R., S. Ottiger, L. Burlini, G. Storti, and M. Mazzotti (2010). Sorption of carbon dioxide, methane and nitrogen in dry coals at high pressure and moderate temperature. International Journal of Greenhouse Gas Control 4(1), 90-101.

[81] Plimpton, S. (1995). Fast parallel algorithms for short-range molecular dynamics. Journal of computational physics 117(1), 1-19.

[82] Rexer, T. F., E. J. Mathia, A. C. Aplin, and K. M. Thomas (2014). High-pressure methane adsorption and characterization of pores in posidonia shales and isolated kerogens. Energy 83 Fuels 28(5), 2886-2901.

[83] Riewchotisakul, S. and I. Y. Akkutlu (2015). Adsorption Enhanced Transport of Hydrocarbons in Organic Nanopores. SPE (September), 28-30.

[84] Roohi, E. and M. Darbandi (2009). Extending the navier-stokes solutions to transition regime in two-dimensional micro-and nanochannel flows using information preservation scheme. Physics of Fluids (1994-present) 21(8), 082001.

[85] Rosenau, P. (1989). Extending hydrodynamics via the regularization of the chapman-enskog expansion. Physical Review A 40(12), 7193.

[86] Roy, S., R. Raju, H. F. Chuang, B. A. Cruden, and M. Meyyappan (2003). Modeling gas ow through microchannels and nanopores. Journal of applied physics 93(8).

[87] Ru, X., Z. Cheng, L. Song, H. Wang, and J. Li (2012). Experimental and computational studies on the average molecular structure of chinese huadian oil shale kerogen. Journal of Molecular Structure 1030, 10-18.

[88] Rullkötter, J. and W. Michaelis (1990). The structure of kerogen and related materials. a review of recent progress and future trends. Organic Geochemistry 16(4), 829-852. 
[89] Saito, R., R. Matsuo, T. Kimura, G. Dresselhaus, and M. Dresselhaus (2001). Anomalous potential barrier of double-wall carbon nanotube. Chemical Physics Letters 348(3), 187-193.

[90] Sakhaee-Pour, A. and S. L. Bryant (2011). Gas permeability of shale. SPE.

[91] Sampath, K. and C. W. Keighin (1982). Factors affecting gas slippage in tight sandstones of cretaceous age in the uinta basin. Journal of Petroleum Technology 34(11), $2715-2720$.

[92] Sanborn, M. J. and R. Q. Snurr (2000). Diffusion of binary mixtures of cf 4 and n-alkanes in faujasite. Separation and purification technology 20(1), 1-13.

[93] Scouten, C., M. Siskin, K. Rose, T. Aczel, S. Colgrove, and R. Pabst Jr (1989). Detailed structural characterization of the organic material in rundle ramsay crossing oil shale. American Chemical Society, Division of Petroleum Chemistry, Preprints;(USA) 34(CONF-8904125-).

[94] Singh, H., F. Javadpour, A. Ettehadtavakkol, H. Darabi, et al. (2014). Nonempirical apparent permeability of shale. SPE Reservoir Evaluation $\mathcal{E}$ Engineering (Preprint).

[95] Skoulidas, A. I., D. M. Ackerman, J. K. Johnson, and D. S. Sholl (2002). Rapid transport of gases in carbon nanotubes. Physical Review Letters 89(18), 185901.

[96] Sone, Y., S. Takata, and T. Ohwada (1990). Numerical analysis of the plane couette flow of a rarefied gas on the basis of the linearized boltzmann equation for hard-sphere molecules. European Journal of Mechanics B Fluids 9, 273-288.

[97] Struchtrup, H. and M. Torrilhon (2003, sep). Regularization of Grad's 13 moment equations: Derivation and linear analysis. Physics of Fluids 15(9), 2668-2680.

[98] Sun, H. (1998). Compass: an ab initio force-field optimized for condensed-phase applications overview with details on alkane and benzene compounds. The Journal of Physical Chemistry B 102(38), 7338-7364.

[99] Taheri (2010). Macroscopic description of rarefied gas flows in the transition regime. Ph. D. thesis.

[100] Talu, O. and A. L. Myers (2001). Molecular simulation of adsorption: Gibbs dividing surface and comparison with experiment. AIChE journal 47(5), 1160-1168. 
[101] Tang, G., W. Tao, and Y. He (2005). Gas slippage effect on microscale porous ow using the lattice boltzmann method. Physical Review E 72(5), 056301.

[102] Tissot, B., B. Durand, J. Espitalie, and A. Combaz (1974). Influence of nature and diagenesis of organic matter in formation of petroleum. AAPG Bulletin 58(3), 499-506.

[103] Travis, K. P., K. E. Gubbins, and N. Carolina (2000). Poiseuille flow of Lennard-Jones fluids in narrow slit pores. The Journal of Chemical Physics 112(4), 1984.

[104] Ungerer, P., J. Collell, and M. Yiannourakou (2014). Molecular modeling of the volumetric and thermodynamic properties of kerogen: Influence of organic type and maturity. Energy $\&$ Fuels 29(1), 91-105.

[105] U.S. Energy Information Administration (2014). The Annual Energy Outlook 2014. U.S. Energy Information Administration.

[106] Vandegrift, G. F., R. E. Winans, R. G. Scott, and E. P. Horwitz (1980). Quantitative study of the carboxylic acids in green river oil shale bitumen. Fuel 59(9), 627-633.

[107] Vandenbroucke, M. (2003). Kerogen: from types to models of chemical structure. Oil \&3 gas science and technology 58(2), 243-269.

[108] Veijola, T. and M. Turowski (2001). Compact damping models for laterally moving microstructures with gas-rarefaction effects. Journal of Microelectromechanical Systems 10(2), 263-273.

[109] Wang, F. P. and R. M. Reed (2009). Pore Networks and Fluid Flow in Gas Shales. In Society of Petroleum Engineers.

[110] Xu, L., M. G. Sedigh, M. Sahimi, and T. T. Tsotsis (1998). Nonequilibrium molecular dynamics simulation of transport of gas mixtures in nanopores. Physical review letters 80(16), 3511.

[111] Yuan, W., Z. Pan, X. Li, Y. Yang, C. Zhao, L. D. Connell, S. Li, and J. He (2014, jan). Experimental study and modelling of methane adsorption and diffusion in shale. Fuel 117, 509519 .

[112] Zhang, T., G. S. Ellis, S. C. Ruppel, K. Milliken, and R. Yang (2012). Effect of organic-matter type and thermal maturity on methane adsorption in shale-gas systems. Organic Geochemistry 47, $120-131$. 
[113] Zhao, Y., Y. Feng, and X. Zhang (2016). Selective adsorption and selective transport diffusion of co2-ch4 binary mixture in coal ultramicropores. Environmental Science $\&$ Technology 50(17), 9380-9389.

[114] Zhu, F., E. Tajkhorshid, and K. Schulten (2002). Pressure-induced water transport in membrane channels studied by molecular dynamics. Biophysical journal 83(1), 154-160.

[115] Zhu, G., L. Liu, Z. Yang, X. Liu, Y. Guo, and Y. Cui (2009). Experiment and mathematical model of gas flow in low permeability porous media. In New Trends in Fluid Mechanics Research, pp. 534-537. Springer Berlin Heidelberg. 